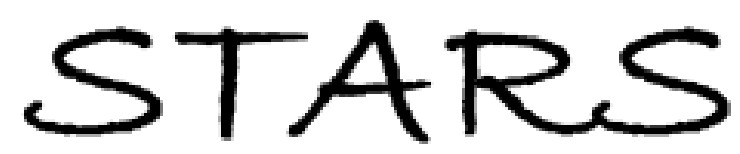

University of Central Florida

STARS

\title{
Supporting situation awareness through robot-to-human information exchanges under conditions of visuospatial perspective taking
}

Elizabeth Phillips

University of Central Florida

Part of the Psychology Commons

Find similar works at: https://stars.library.ucf.edu/etd

University of Central Florida Libraries http://library.ucf.edu

This Doctoral Dissertation (Open Access) is brought to you for free and open access by STARS. It has been accepted for inclusion in Electronic Theses and Dissertations, 2004-2019 by an authorized administrator of STARS. For more information, please contact STARS@ucf.edu.

\section{STARS Citation}

Phillips, Elizabeth, "Supporting situation awareness through robot-to-human information exchanges under conditions of visuospatial perspective taking" (2016). Electronic Theses and Dissertations, 2004-2019. 4936.

https://stars.library.ucf.edu/etd/4936

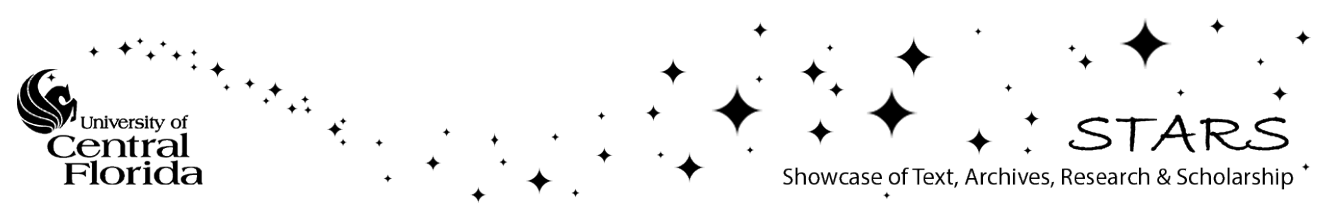


SUPPORTING SITUATION AWARENESS THROUGH ROBOT-TO-HUMAN INFORMATION EXCHANGES UNDER CONDITIONS OF VISUOSPATIAL PERSPECTIVE TAKING

by

ELIZABETH KATHLEEN PHILLIPS

B.S. University of Central Florida, 2008

M.A. University of Central Florida, 2013

A dissertation submitted in partial fulfillment of the requirements

for the degree of Doctor of Philosophy

in the Department of Psychology

in the College of Sciences

at the University of Central Florida

Orlando, Florida

Spring Term

2016

Major Professor: Florian G. Jentsch 
(C) 2016 Elizabeth Kathleen Phillips 


\begin{abstract}
The future vision of military Soldier-robot teams is one in which Soldiers and robots work together to complete separate, but interdependent tasks that advance the goals of the mission. However, in the near term, robots will be limited in their ability to successfully perform tasks without, at least, occasional assistance from their human teammates. A need exists to design, in robots, mechanisms that can support human situation awareness (SA) regarding the operations of the robot, which humans can use to provide interventions in robot tasks. The purpose of the current study was to test the effects of information exchanges provided by a robot on the development of SA in a human partner, under differing levels of visual perspective taking, and the consequential effects on the quality of human assistance provided to a robot. After data screening, fiftysix male participants ranging in age from 18 to $29(M=18.89, S D=3.412)$ were included in the analysis of the results. Hierarchical multiple regression and a series of ANOVAs with comparisons between individual within-subjects study conditions were conducted to analyze five Hypotheses. The results of this study revealed that if robots, through robotto-human information exchanges, can increasingly support a human's understanding of when assistance is needed, humans will be better able to provide that assistance. As opposed to originally hypothesized, this study also showed that fewer instances in which robots share status information with their human counterparts may be more beneficial for supporting awareness, assistance, and dual task performance than more information sharing, by guarding against performance decrements that could be the result of receiving
\end{abstract}


too many robot-to-human information exchanges. It was also thought that anchoring robot-to-human information sharing with spatial information in reference to the human's view of the environment would be most beneficial for supporting awareness regarding the robot and assistance provided to the robot. This notion was not supported. Instead, results suggested that if extra spatial information is added to robot-to-human information exchanges, representing that spatial information in reference to a cardinal, global-relative perspective of the environment may be better for supporting awareness and assistance than representing that information in reference to the human's view of the environment. 
For Daniel, my favorite 


\section{ACKNOWLEDGMENTS}

It's rarely true that people achieve great things on their own. I am extremely grateful for a number of people who supported me in this endeavor. Thank you to the members of my dissertation committee: Randall Shumaker, Valerie Sims, Clint Bowers, and my academic advisor and director of the Team Performance Lab (TPL), Florian Jentsch. Under your guidance, TPL has done more for my personal and professional development than I thought possible when I started on this path. I never imagined that I would get this far, and I am forever grateful for the opportunities you have afforded me.

To my lab colleagues and research assistants, Andrew (Thank you for shouldering the extra work so that I could finish this thing. It did not go unnoticed), Javier, Camilo, Karissa, Caitlin, Audrey, Lauren, Amanda, Staci, and MiAmor, I could not have done this without your support and your smiles. Thank you.

I have also been exceedingly fortunate to have had a number of peer mentors who looked out for me along the way. Joe Keebler (you gave me my first research position and started me on this path, Thank you), Scott Ososky (Thank you for allowing me to flourish under your guidance. Also, the countless walks, and coffee were super helpful and much appreciated), Dave Schuster, Mike Curtis, Liz Lazzara, Tom Alicia, Lauren Benishek, Grant Taylor, and many others, thank you for your investment in my wellbeing and unending support. I am honored to call you my friends. 
Dan, thank you for sticking by me every time I thought I had "Only two years left" and each time I said, "This is going to be a tough semester"...every single semester. Seriously though, I appreciate your patience (and hugs), and I look forward to completing many, many more journeys with you. Perhaps we can start a collection of spores, molds, and fungi soon.

There is no way that I could have come this far without the support of my family. Garrett, Kaitlin, Tim, Frank, and the Pellitos, I love you. Mom and Dad, thank you for the many sacrifices that enabled me to have so much. Grandma, thank you for setting in motion a long history of capable women in our family.

Finally, I would be remiss if I did not acknowledge the fact that I have had a legion of public school teachers who invested countless, thankless, tragically underfunded hours in the education and development of all students, not just me. I appreciate all of those hours.

Thank you all. I look forward to sharing with you whatever comes next.

The research reported in this document was performed in connection with Contract Number W911NF-10-2-0016 with the U.S. Army Research Laboratory. The views and conclusions contained in this document are those of the authors and should not be interpreted as presenting the official policies or position, either expressed or implied, of the U.S. Army Research Laboratory, or the U.S. Government unless so designated by other authorized documents. Citation of manufacturer's or trade names does not 
constitute an official endorsement or approval of the use thereof. The U.S. Government is authorized to reproduce and distribute reprints for Government purposes notwithstanding any copyright notation heron. 


\section{TABLE OF CONTENTS}

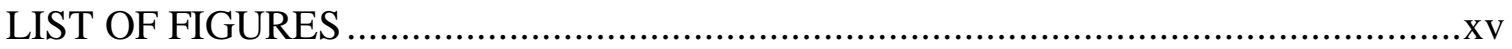

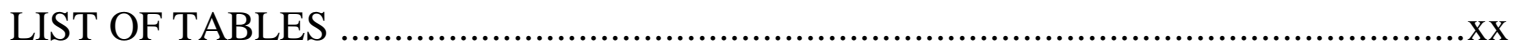

CHAPTER ONE: INTRODUCTION …............................................................. 1

Human assistance provided to robots....................................................

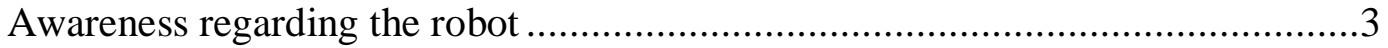

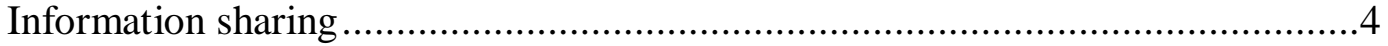

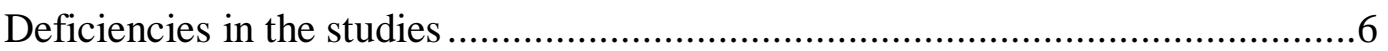

Integration of visuospatial perspective ..................................................

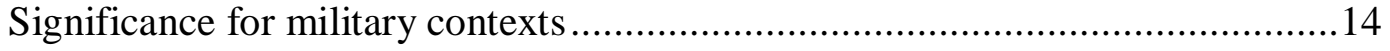

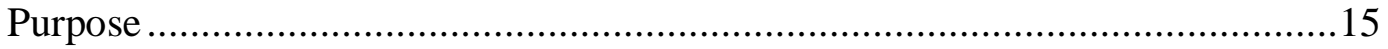

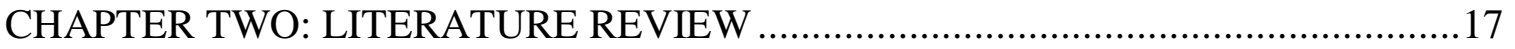

Situation awareness and human assistance provided to a robot .......................17

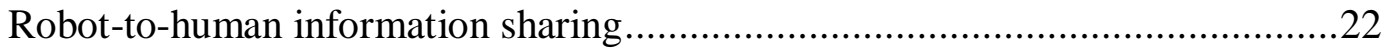

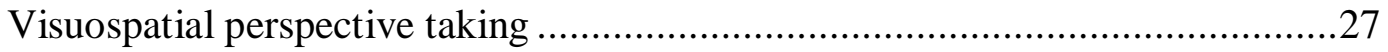

Spatial skills and situation awareness ..............................................29

Spatial skills and performance in human-robot teams ..........................31

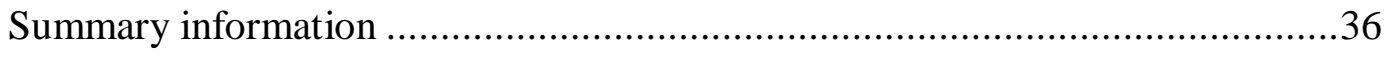




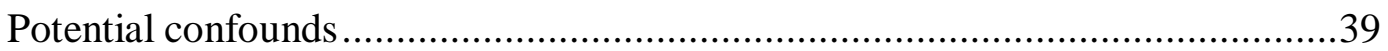

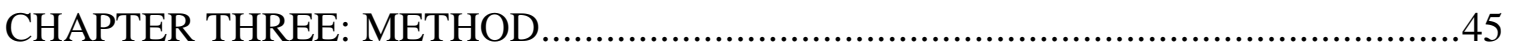

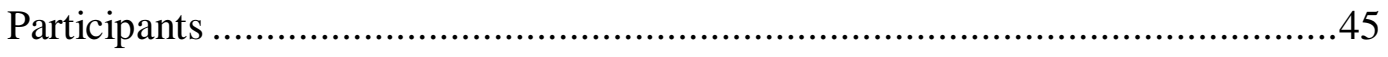

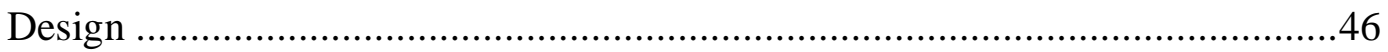

Experimental task .......................................................................4 47

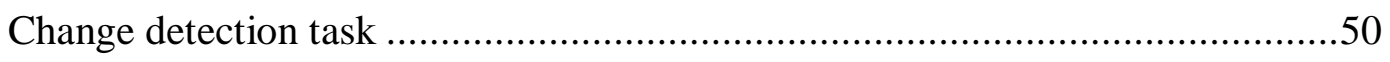

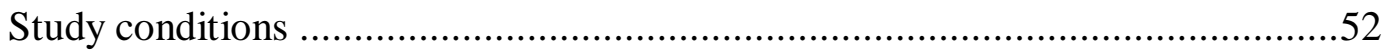

Waypoint status updates ( 7 updates) condition ....................................52

Waypoint status updates (13 updates) condition................................53

Traveling +waypoint status updates conditions with Level 1 VPT condition

Traveling +waypoint status updates conditions with Level 2 VPT condition

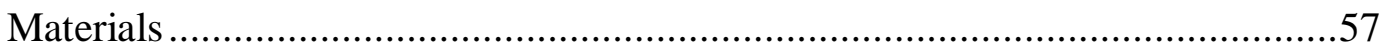

Simulation testbed and experimental apparatus..................................57

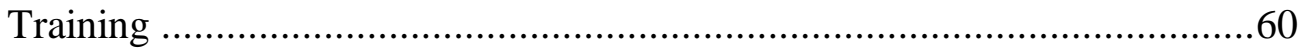

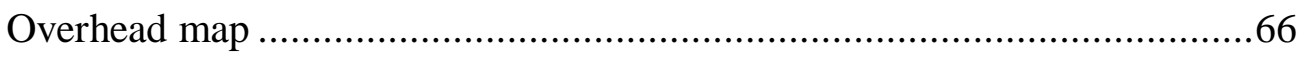

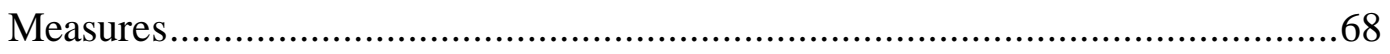




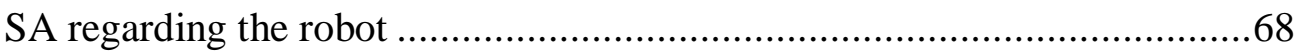

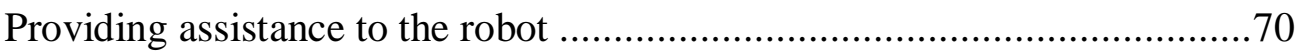

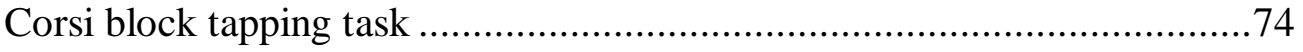

Perspective taking /Spatial Orientation Test ................................................75

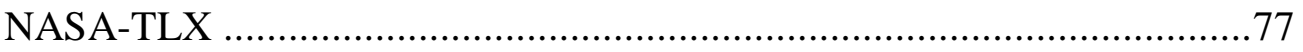

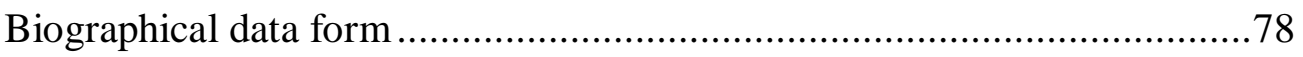

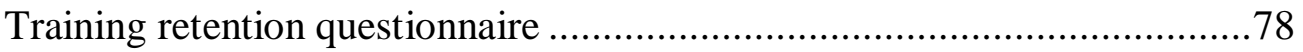

Change detection task performance ........................................................79

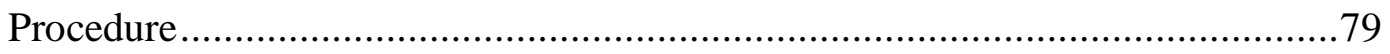

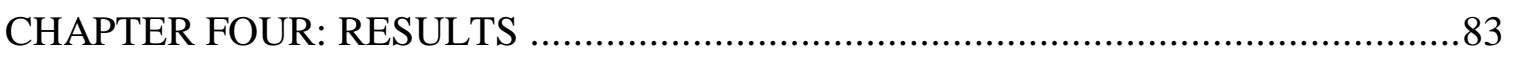

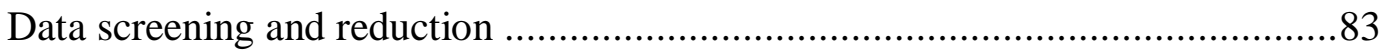

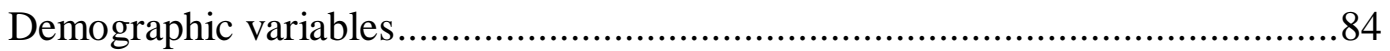

Preliminary analyses: Demographic variables and assignment to order conditions

Preliminary analyses: Dependent variables and assignment to order conditions ..86

Preliminary analyses: Mental workload and assignment to order conditions ........86

Preliminary analyses: Mental workload across within-subjects conditions ..........87

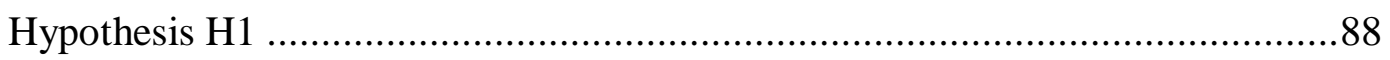




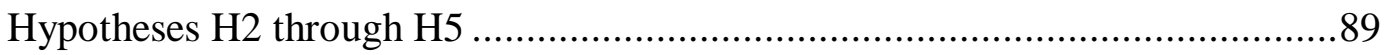

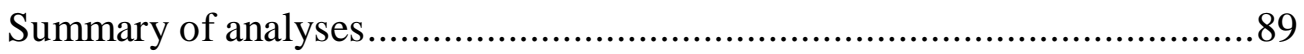

Hypotheses two and four: SA related hypotheses .......................................90

Hypothesis H2 contrast.................................................................91

Hypothesis H4 contrast...............................................................91

Additional contrast 1 .................................................................. 92

Hypotheses three and five: Providing assistance to the robot hypotheses ...........93

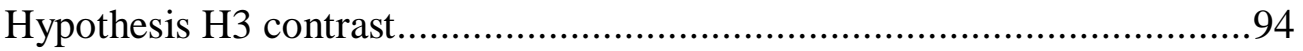

Hypothesis H5 contrast..............................................................94

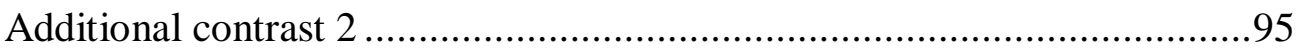

Additional analyses: Performance on change detection task ...........................96

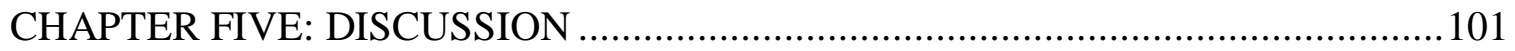

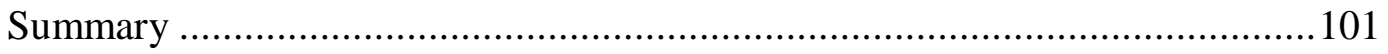

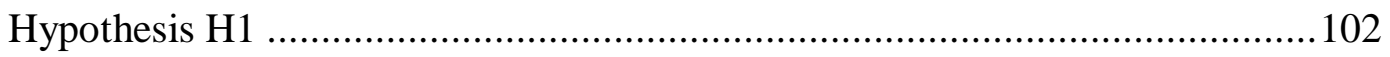

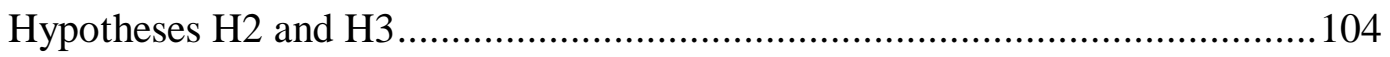

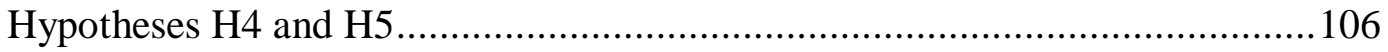

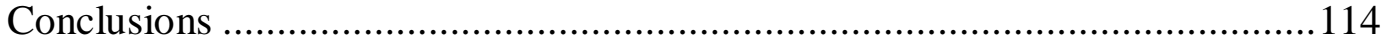

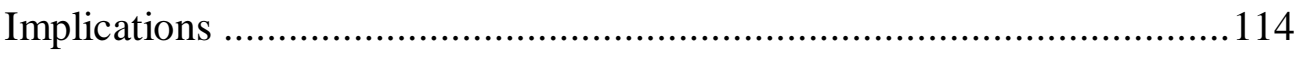


Limitations of the study.

Areas for future research

APPENDIX A: UCF APPROVAL OF HUMAN-SUBJECTS RESEARCH . 124

APPENDIX B: U.S. ARMY HUMAN RESEARCH PROTECTIONS OFFICE

HEADQUARTERS-LEVEL ADMINISTRATION REVIEW

APPENDIX C: GRAPHS OF HYPOTHESIZED RESULTS

APPENDIX D: GRAPHICAL REPRESENTATIONS OF EXPERIMENTAL

SCENARIOS 135

APPENDIX E: OVERHEAD MAPS PROVIDED TO PARTICIPANTS 143

APPENDIX F: REROUTING MAPS PROVIDED TO PARTICIPANTS. 151

APPENDIX G: ROBOT-TO-HUMAN INFORMATION EXCHANGES FOR

EXPERIMENTAL MISSION SCENARIOS 164

APPENDIX H: PLANNED ANALYSES AND EXPECTED PATTERNS OF RESULTS 190

APPENDIX I: REMOVAL OF FEMALES IN THE ANALYSES. .205

APPENDIX J: INFORMED CONSENT.

APPENDIX K: POST-PARTICIPATION INFORMATION.

APPENDIX L: PARTICIPANT TRAINING SLIDES

APPENDIX M: PARTICIPANT TRAINING SLIDE SCRIPT .248 
LIST OF REFERENCES ........................................................................ 260 


\section{LIST OF FIGURES}

Figure 1. The Soldier issues the screen the back door command. Then, the robot and the Soldier begin to move to their respective observation posts. Figure includes a graphical representation of the intended paths to the observation posts.

Figure 2. While navigating to the observation post, the robot alerts the Soldier that there is an abandoned car blocking the path, representing an exchange of Level $1 \mathrm{SA}$ information. Figure includes a graphical representation of the obstructed path.

Figure 3. The robot alerts the Soldier that a new path to the observation post has been generated. Figure includes a graphical representation of the revised path. .11

Figure 4.The robot alerts the Soldier that the new path will take the robot around the building to the robot's left, in the direction of the Soldier's right. Figure includes graphical representation of the robot's revised path.

Figure 5.The Soldier realizes that the robot's revised path is undesirable and makes a correction. Figure includes a graphical representation of the Soldier's revision to the robot's path.

Figure 6. Conceptual mapping of levels of VPT to levels of SA regarding the robot.

Figure 7. Graphical representation of Hypothesis H1.

Figure 8. Graphical representation of Hypotheses $\mathrm{H} 1, \mathrm{H} 2$, and $\mathrm{H} 3$, including the mediating effect of SA regarding the robot on the relationship between robot-to-human information exchanges and human assistance provided to the robot. 
Figure 9. Graphical representation of Hypotheses H1, H2, H3, H4, and H5. .....35

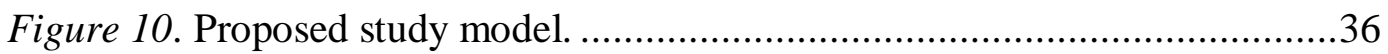

Figure 11. Graphical representation of two Latin squares that were used to determine the order in which participants completed each mission associated with each study condition.

Figure 12. Example of a robot-to-human information exchange in the form of a waypoint status update .49

Figure 13. Example of a robot-to-human information exchange in the form of a traveling status update, given while the robot was maneuvering between waypoints. .....50

Figure 14. Example of the overhead map and icons displayed for the change

detection task.

Figure 15. Examples of Level 1 and Level 2 VPT context added to traveling updates.

Figure 16. Screen shot of the OCU in the MIX simulation testbed. Note, the overhead map displayed at the bottom portion of the OCU was not an overhead view of the robot's environment or the robot's waypoint route.

Figure 17. Photograph of the physical experimental apparatus. .60

Figure 18. Example of training materials for the compass rose. 63

Figure 19. Example of training materials for the clock face directions. 
Figure 20. Overhead map used for the mission associated with the 13UPLevel2 condition. Blue circles represent the robot's planned waypoint route. The start and end points of the route are denoted. Note: Map was printed on $8 \frac{1}{2} 2 \times 14$ legal size paper......68

Figure 21. Rerouting map provided to participants during the 13UPLevel2 condition. The blue circle depicts planned waypoint number 3 in the robot's planned waypoint path

Figure 22. Example of a rerouting drawing completed in 13UPLevel2 condition. The blue circle depicts planned waypoint number 3 in the robot's planned waypoint path. The red X denotes the robot's location at the time of the probe event. The route in purple denotes a rerouting path that participants could have drawn. ................................. 72

Figure 23. Example of Corsi block tapping task stimuli. .................................75

Figure 24. Example item from the Perspective taking/Spatial orientation test.....76

Figure 25. Flow chart of study procedure .............................................. 82

Figure 26. Pattern of mean scores on the measure of SA across within-subjects conditions and planned contrasts

Figure 27. Pattern of mean scores on the measure of providing assistance to the robot across within-subjects conditions and planned contrasts

Figure 28. Pattern of mean scores and post-hoc comparisons on the change detection task across within-subjects conditions and planned contrasts. 
Figure 29. Overhead map provided to participants in the Traveling + waypoint status updates with Level 1 VPT context condition, experimental mission 3. Note the compass rose contains cardinal directions only.

Figure 30. Overhead map provided to participants in the Traveling + waypoint status updates with Level 2 VPT context condition, experimental mission 4. Note the compass rose contains both cardinal and clock face directions.

Figure 31. Contrast of scores on the measure of SA regarding the robot in Missions 3 and 4

Figure 32. Contrast of scores on the measure of human assistance provided to the robot in Missions 3 and 4.

Figure 33. Contrast of scores on the measure of SA regarding the robot in Missions 1 and 2. 193

Figure 34. Contrast of scores on the measure of human assistance provided to the robot in Missions 1 and 2. 194

Figure 35. Contrast of scores on the measure of SA regarding the robot in Missions 2 and 3 196

Figure 36. Contrast of scores on the measure of human assistance provided to the robot in Missions 2 and 3. 196

Figure 37. Contrast of scores on the change detection task in Missions 1 and 2. 
Figure 38. Contrast of scores on the change detection task in Missions 3 and 4.

Figure 39. Contrast of scores on the change detection task in Missions 2 and 3.

Figure 40. Contrast of scores on the change detection task in Missions 3 and 4.

Figure 41. Conceptual representation of overall pattern of results for scores on the measure of SA regarding the robot.

Figure 42. Conceptual representation of overall pattern of results for scores on the measure of human assistance provided to the robot. 202

Figure 43. Conceptual representation of overall pattern of results for scores on the change detection task. .203

Figure 44. Expected positive relationship between SA regarding the robot and human assistance provided to the robot. .204 


\section{LIST OF TABLES}

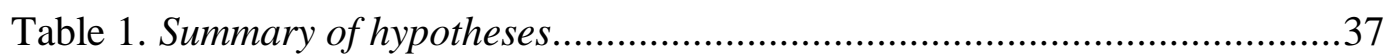

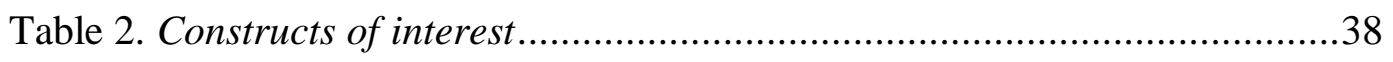

Table 3. Table of potential confounding variables .........................................44

Table 4. Table of within-subjects conditions. All participants completed

conditions numbered 1, 2, 3, and 4 .

Table 5. Table of SA regarding the robot questions ........................................70

Table 6. Table listing instructions for scoring each rerouting the robot map. .....73

Table 7. Descriptive statistics and inter-correlations among demographic

variables.

Table 8. Table of means and standard deviations for scores on the NASA-TLX

measure.

Table 9. Summary of comparisons conducted to analyze Hypotheses H2 through

H5.

Table 10. Table of means and standard errors for SA regarding the robot scores

across study conditions.

Table 11. Table of means and standard errors for scores on the measure of

providing assistance to the robot across study conditions.

Table 12. Table of means for scores on the change the detection task across study

conditions. 
Table 13. Table of mean scores on the change detection task for one minute of performance across study conditions and the practice task .........................................100

Table 14. Table of Pearson's correlations $(N=76)$ between individual difference variables and dependent variables for both males and females in the study.

Table 15. Mean scores for males and females on the measure of SA across experimental mission scenarios. 


\section{CHAPTER ONE: INTRODUCTION}

The long-term vision of military robotics is one in which robots will serve as integrated members of dismounted Soldier-robot (SR) teams, working in complex battlefield environments, and collaborating with Soldiers to achieve common goals. Robots will be expected to extend the skills of Soldiers and engage in behaviors that resemble those employed in high-performing human-human teams. Autonomous capabilities will allow robots to complete separate but interdependent tasks, without constant direct oversight from Soldiers. However, although future military robots will be more autonomous than today's largely teleoperated robotic systems, they will not perfectly replicate Soldiers in reliability (i.e., ability of the robot to avoid failures and complete tasks alone) or intelligence (i.e., robot's ability to be aware of its failures and make appropriate adjustments), at least in the near term. Instead, robots will possess a subset of capabilities that will be leveraged to perform a wide variety of work, not unlike the ways in which working animals are utilized in human - animal teams (Phillips, Ososky, Swigert, \& Jentsch, 2012). Just like working animals, military robots will be skilled at performing some tasks and limited in performing others, and, as a result, will require occasional human assistance to complete tasks.

\section{Human assistance provided to robots}

Because robotic teammates in the near future will be limited in reliability, the Soldier — robot team will need a means to recover from robot failures or deal with robots 
that are unable to complete their tasks alone. In programs such as the U.S. Army's Robotics Collaborative Technology Alliance (RCTA), "Screen-the-back-door" (STBD) is a common vignette used to describe tasking of humans and robots in future SR teams. In this scenario, the robotic team member is tasked with navigating to, maintaining persistent surveillance of, and reporting the activities at, the back door of a suspicious building (Army Research Laboratory [ARL], 2012). At the same time, the human is tasked with recognizing the suspicious nature of the building, issuing the "Screen-theback-door" command to the robot, moving the remainder of the team to a safe location, and deciding on the next course of action.

In the near term, robotic competence for completing STBD tasks will not match human competence. Although the robotics and machine learning communities are working to develop human-level competence, robots still have difficulty with skills that come naturally to humans. For example, robots have difficulty with perceptual tasks like perception-based autonomous navigation, as well as environmental perception, especially in cluttered or complex terrain (Fong, Thorpe, \& Baur, 2003; Jackel, Hackett, Krotkov, Perschbacher, Pippine, \& Sullivan, 2007; Nguyen-Huu, Titus, Tilbury, \& Ulsoy, 2009).

In the STBD vignette above, the robot may need assistance completing a number of subtasks that require perception to execute the command. For instance, once the robot has navigated to the back door of the building, the Soldier may need to configure the robot's view of the back door, or, in the event of multiple back doors, specify the door to 
surveil. Researchers Fong, Thorpe, and Baur (2003) described that assistance offered by humans can often make a big impact on/for robots, "frequently, the only thing the robot needs to get out of difficulty and to perform better is some advice (even a small amount) from a human" (p. 255). However, as they currently exist, robots are not equipped to know when assistance from a human is needed, or when robots are performing poorly. This is especially problematic as robot performance tends to be brittle when faced with novel applications or dynamic environments (McCarthy, 1995; Novianto \& Williams, 2009).

\section{Awareness regarding the robot}

For the future of Soldier-robot teams, there will be a need to reconcile the requirement for humans to provide assistance to robots, with limitations in the ability of robots to know when they need assistance and subsequently, ask for it. To remedy this problem, robots will need to be designed in ways that promote a human's awareness of when to intervene and what type of assistance to offer. In today's state-of-the-art robots (SOA), in which continuous monitoring and control are the prevalent methods of human-robot interaction, detecting robot failures is achieved through "the eyes of the robot" (see Burke, Murphy, Coovert, \& Riddle, 2004; Casper \& Murphy, 2003; Yanco \& Drury, 2007). These teleoperated robots provide a continuous view of their actions through constantly updated video data that are sent back to operators. However, this view of the robot comes at high costs to robot operators (e.g., loss of perceptual cues in the 
environment, cognitive fatigue, as well as inability to control the robot alone, Burke \& Murphy, 2004; Burke, Murphy, Coovert, \& Riddle, 2004)

Until robots can be engineered to complete tasks with near-perfect reliability and much greater competency, there will be a need to facilitate human awareness of when to intervene in robot tasks, without continuous monitoring or prompting for help from the robot. As a result, a research need exists to determine how the skills of robots can be leveraged to facilitate operator awareness of the robot, and consequently, assistance in robot tasks.

\section{Information sharing}

One method of facilitating operator awareness of robot tasks is through robot-tohuman information sharing. Specifically, Schuster and Jentsch (2012) stated that robots can be active participants in the development of Soldiers' situation awareness (SA) through their communication and coordination with Soldiers. However, under conditions in which robots possess high autonomy and self-sufficiency the constant need for communication between robots and humans is removed. As a consequence, in those situations, the operations of robots can become opaque to human teammates. When a robot periodically exchanges information with a person, that person can stay apprised of the status of the robot and its surrounding environment, as interactions can be a means to foster awareness of the activities of each member in a team (Salmon, Stanton, Walker, \& Jenkins, 2009). 
Many of the prior approaches to facilitating operator situation awareness regarding the robot (i.e., the perception of the robot's location, surroundings, and status; the comprehension of their meaning; and the projection of how the robot will behave in the near future; Yanco \& Drury, 2004) ${ }^{1}$, have centered on developing and improving control and display devices that serve as the medium for information exchanges between the robot and the human operator. Specifically, research has focused on evaluating various displays and interfaces for supporting situation awareness for the remote teleoperation of robots. In most instances of teleoperation, robots provide a continuous view of their actions via video data that are sent back to operators as a continuous stream of information. As such, video is the primary source of information about the status of the robot and its operating environment (Yanco \& Drury, 2007).

This method of robot-to-human information exchange is taxing on the human visual and information processing systems, and can lead to human performance issues, including loss of peripheral perception of the environment due to a limited field of view (FOV); loss of the ability to orient the robot in the environment due to unawareness of the robot's inclination and shape; loss of depth perception which leads to an underestimation of distance to and size of targets (Chen, Haas, \& Barnes, 2007); as well as cognitive

\footnotetext{
${ }^{1}$ Briefly, the most widely accepted definition of situation awareness is a three-level model given by Endsley (1995a) as

The perception of elements in the environment within a volume of time and space (Level 1), the comprehension of their meaning (Level 2), and the projection of their status in the near future (Level 3) (p. 36).

Situation awareness regarding the robot is Endsley's (1995a) three-level model applied specifically to the robot.
} 
fatigue. As a result, it becomes very difficult for operators to build and maintain situation awareness regarding the robot and its operating environment. For instance, Murphy and Burke (2005) described that, for teleoperated robots, roughly $60 \%$ of communications between operators were related to building and maintaining SA, and that almost half of the time on task was dedicated to operators trying to determine what they were seeing through the eyes of the robot (as opposed to spending time actively maneuvering the robot). Consequently, many researchers (see Chen, Haas, \& Barnes, 2007) have investigated means to reduce workload on the visual system and increase situation awareness regarding the robot through improvements to robot-to-human information exchange media like display devices and interaction interfaces (e.g., supplementing video from the robot with north-up, or track-up maps; outfitting robots with multiple or wideangle cameras to increase FOV).

\section{$\underline{\text { Deficiencies in the studies }}$}

Much of the existing work seeks to improve information available to the operator by improving the device through which humans receive information exchanges from robots. A primary problem with the continued use of display-based interfaces for information exchange in teleoperated tasks, is that they require operators to be "heads down" while engaged in continuous monitoring of a video display (Brown, Gray, Blanco, Juneja, Alberts, \& Reinerman, 2011). Even if changes to the display device reduce workload on the human visual system and improve SA regarding the robot, the use of the 
device still requires operators to keep their hands and eyes on the device when engaged in teleoperating the robot. This type of "heads down" operation of the robot can reduce the effectiveness of a squad of soldiers, as the robot operator is not able to keep "hands on" his/her weapon and "eyes on" the environment. Consequently, the operator requires protection from one or more of his/her other teammates (Brown et al., 2011). As such, the intended design of future military robotic teammates aims to eliminate the need for continuous monitoring and display-based interaction with the robot (ARL, 2012).

At the same time, the elimination of a visual display device presents a hurdle for facilitating operator SA regarding the robot, as many of the findings on improving SA regarding the robot through the information exchange devices, will not be applicable to future military robots. Visual display devices for continuous monitoring will largely be absent. As a result, there exists a gap in the research concerning means to facilitate operator SA regarding the robot when continuous monitoring or displayed based interaction with the robot is removed. Under the current model of robot teleoperation, SA regarding the robot is improved by maps, sensor overlays, and other contextual details that are synthesized and presented to operators through the visual display. With the requirement that devices be removed, there is a need to investigate other means to facilitate SA regarding the robot, which will ultimately enable operator intervention in robot tasks. 


\section{Integration of visuospatial perspective}

One potential solution is to improve/augment information that is transferred to humans directly from the robot. A robot may be able to synthesize and augment information about the environment via its own internal processing, and transfer that information in its augmented form directly to the human teammate(s) (e.g., via text, speech, tactile, or other appropriate medium). For example, the ability of the robot to interpret and integrate situational views, and transmit that information to human partners could aid the human in developing SA regarding the robot and its operating environment. The ability to apply visuospatial perspective taking (VPT) (Piaget \& Inhelder, 1956 as cited in Flavell, 1992) to information exchanges could influence the development of SA regarding the robot.

In general, VPT refers to the ability to imagine how a scene looks from varying viewpoints. VPT is said to have two levels; Level 1 refers the ability to discern whether an object in the environment will be visually accessible to an outside observer. Level 2 refers to the ability to discern not only whether said object is visually accessible, but also how that object will look from an outside observer's point of view if the object is visually accessible or were to become visually accessible (Flavell, 1992).

To illustrate, in the STBD vignette, the Soldier and the robot begin the scenario in a common starting position. The Soldier issues the "Screen the back door" command, and 
both teammates begin to navigate to their respective observation posts (OP); the robot's $\mathrm{OP}$ is near the rear door, whereas the Soldier's OP is near the front door (see Figure 1).

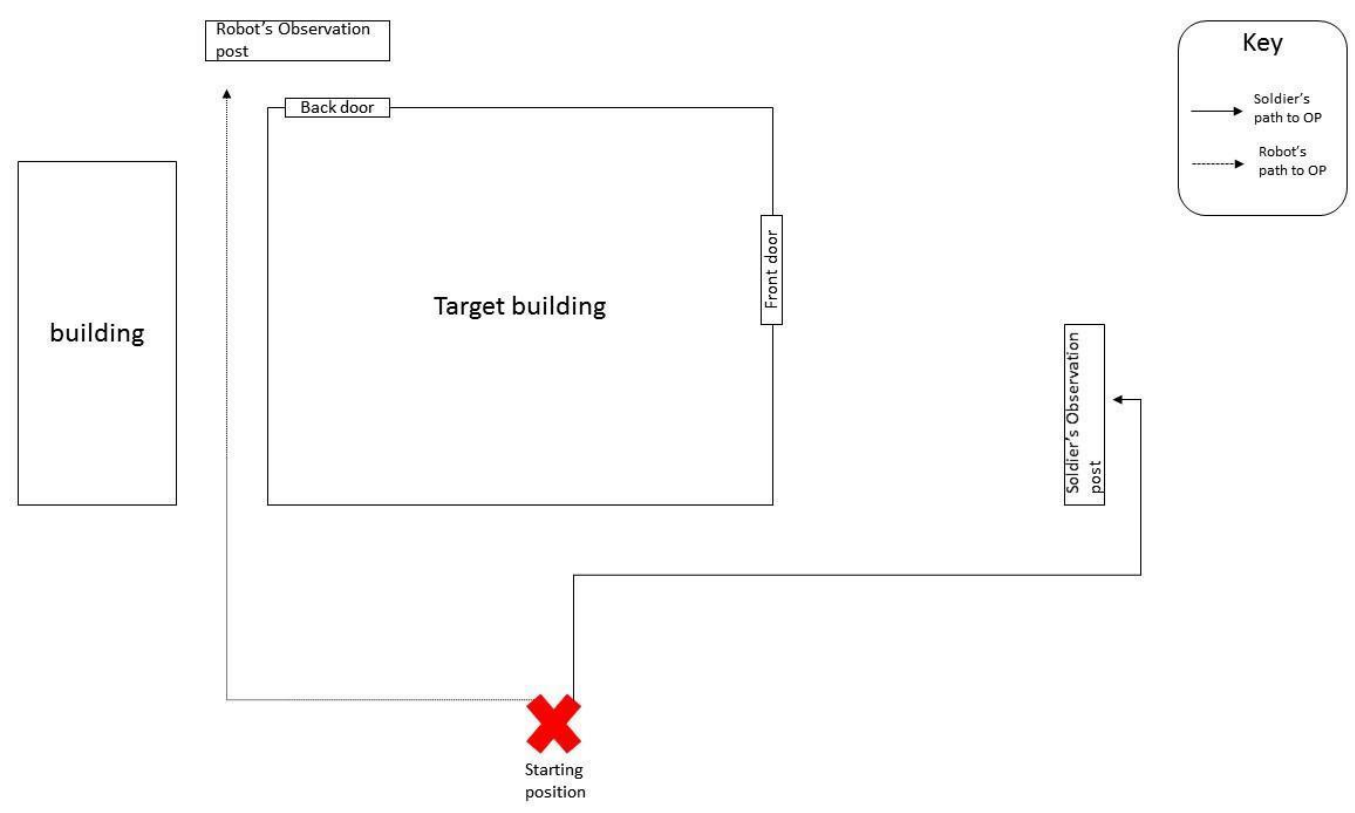

Figure 1. The Soldier issues the screen the back door command. Then, the robot and the Soldier begin to move to their respective observation posts. Figure includes a graphical representation of the intended paths to the observation posts.

While the robot is navigating to the back door of the building, the robot sends an alert message to the Soldier that there is an abandoned car located along its intended route to the back door of the building (see Figure 2). This notification is an exchange of Level $1 \mathrm{SA}$ information regarding the robot (i.e., information that is related to the perception of relevant features of the robot's environment, as described by Endsley's, 1995a, 3-level model of SA). 


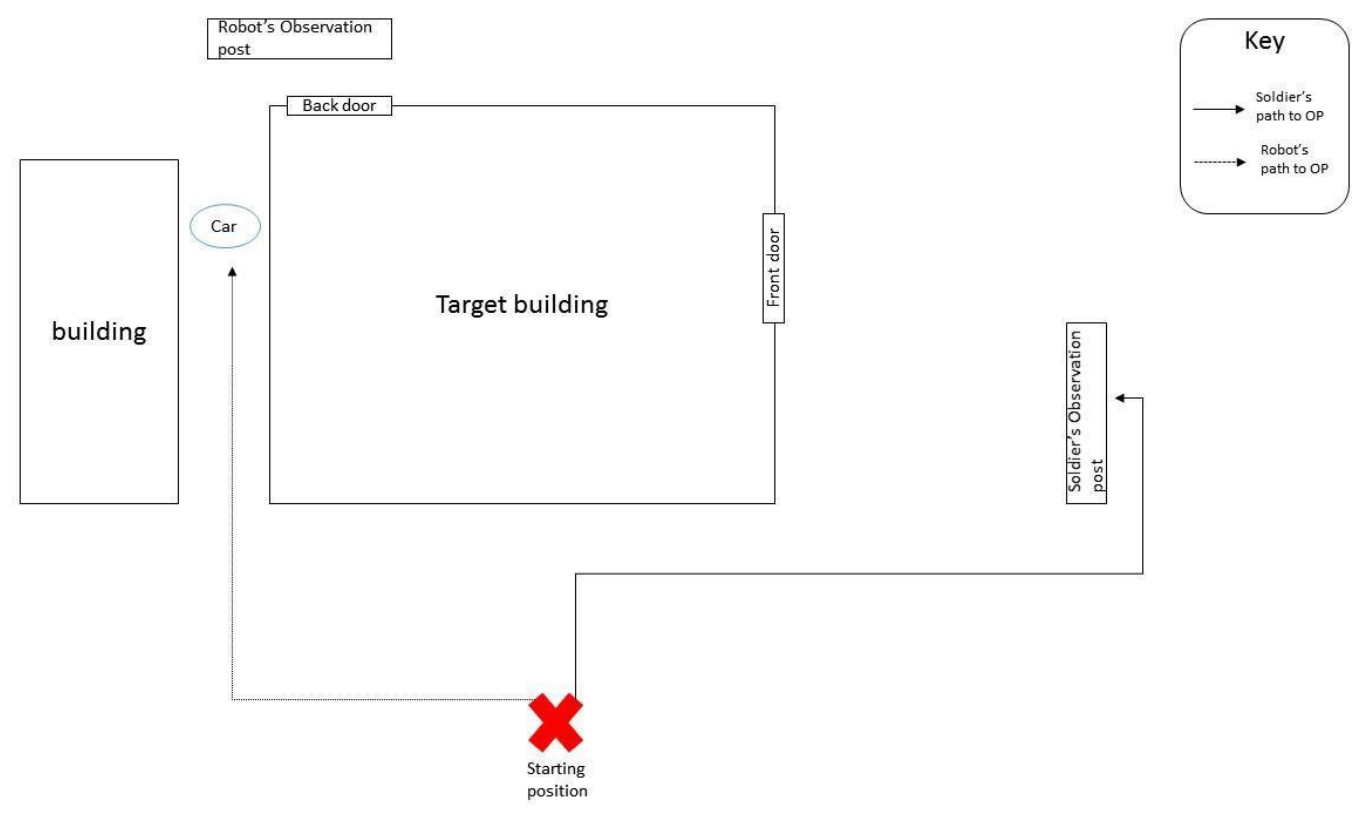

Figure 2. While navigating to the observation post, the robot alerts the Soldier that there is an abandoned car blocking the path, representing an exchange of Level $1 \mathrm{SA}$ information. Figure includes a graphical representation of the obstructed path.

The exchange includes inferences concerning the visual accessibility of objects to others (i.e., Level 1 visuospatial perspective taking), as the robot has made an alert to the Soldier based on its knowledge that the Soldier does not have visual access to the car located along the robot's navigation route. Due to the obstruction in the original path, the robot then alerts the Soldier that its path planner has suggested a new route to the OP (see Figure 3). This notification is an exchange of Level $2 \mathrm{SA}$ information regarding the robot (i.e., information that is related to the meaning of relevant features of the environment; Endsley, 1995) as the position of the car necessitates a new route. This exchanges also includes Level 1 VPT. 


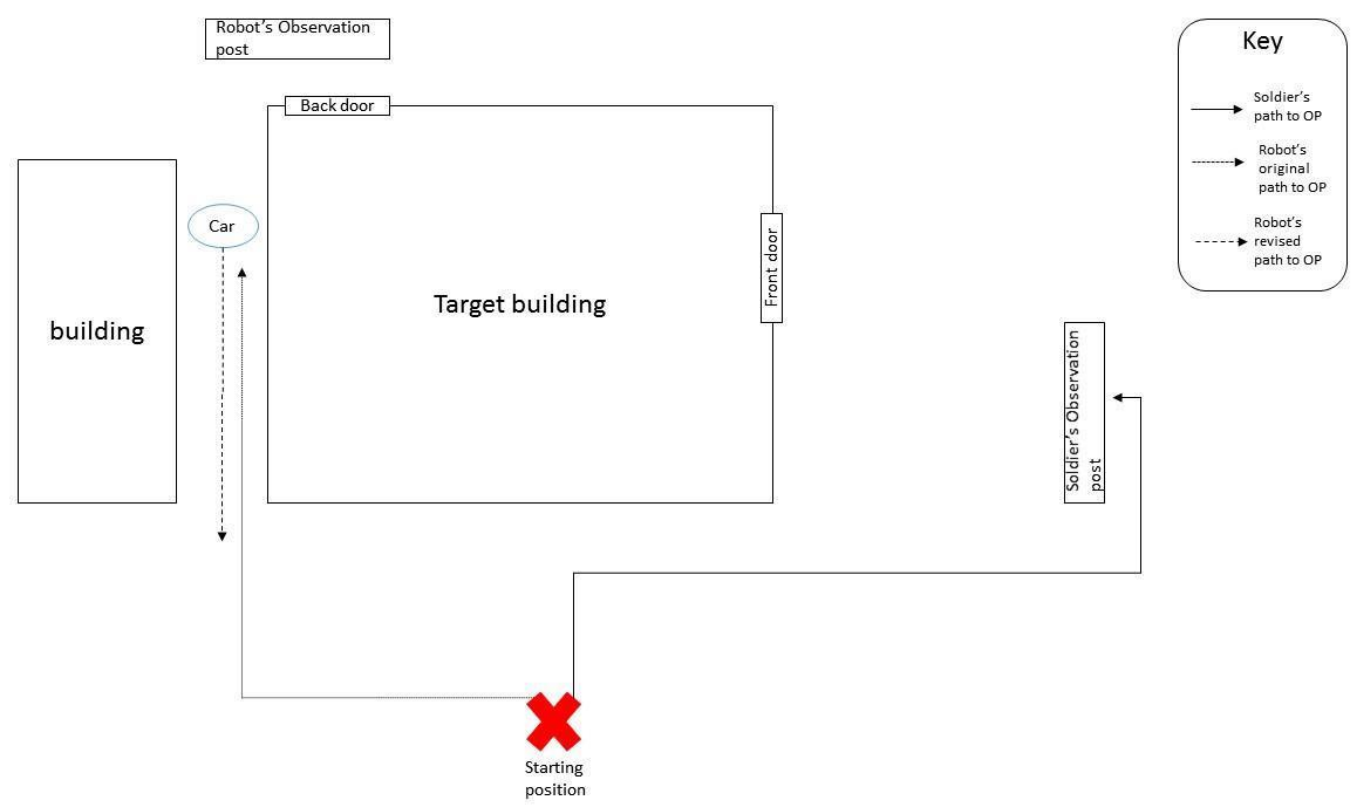

Figure 3. The robot alerts the Soldier that a new path to the observation post has been generated. Figure includes a graphical representation of the revised path.

Although the previous update is likely useful to the Soldier, the robot may be able to foster a deeper level of SA in the Soldier (i.e., Level $3 \mathrm{SA}$, the projection of future states), if the robot is also able to transform the information from Level 1 visuospatial perspective information to Level 2 visuospatial perspective information. Level 2 visuospatial perspective taking refers to the ability to infer how objects appear differently from different spatial points of view (Flavell, 1992). For instance, due to the obstruction in the robot's original path, the robot could alert the Soldier that its path planner has suggested a new route to the OP, and that the path will take the robot to the left around the building, in the direction of the Soldier's right (Level 2 VPT). This additional 
information would provide the Soldier with a means to make a projection about the future path of the robot (see Figure 4).

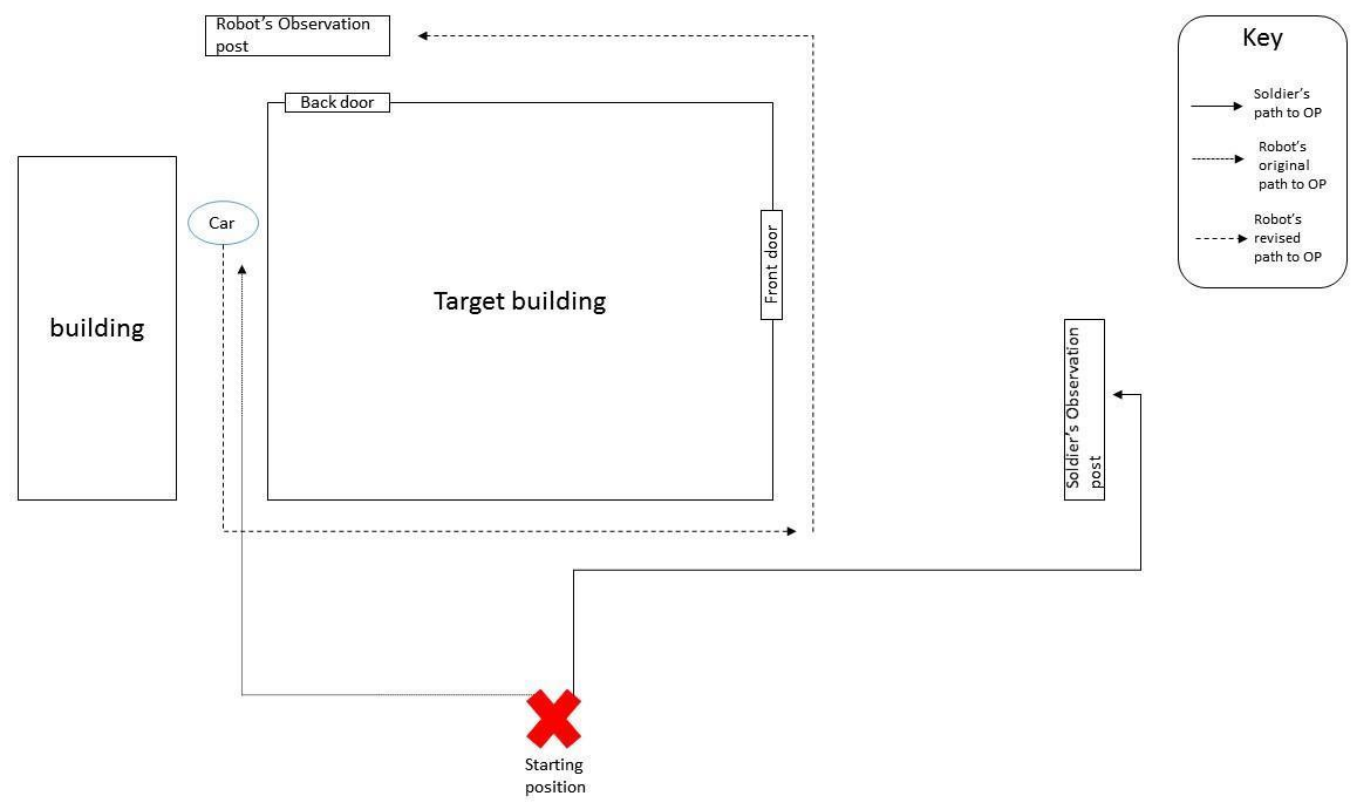

Figure 4.The robot alerts the Soldier that the new path will take the robot around the building to the robot's left, in the direction of the Soldier's right. Figure includes graphical representation of the robot's revised path.

Consequently, the suggested path will place the robot in direct line of sight with one of the main doors of the building. Realizing this new path may place the robot at risk of being detected, or possibly destroyed, the Soldier can make a correction to the robot's path (see Figure 5). 


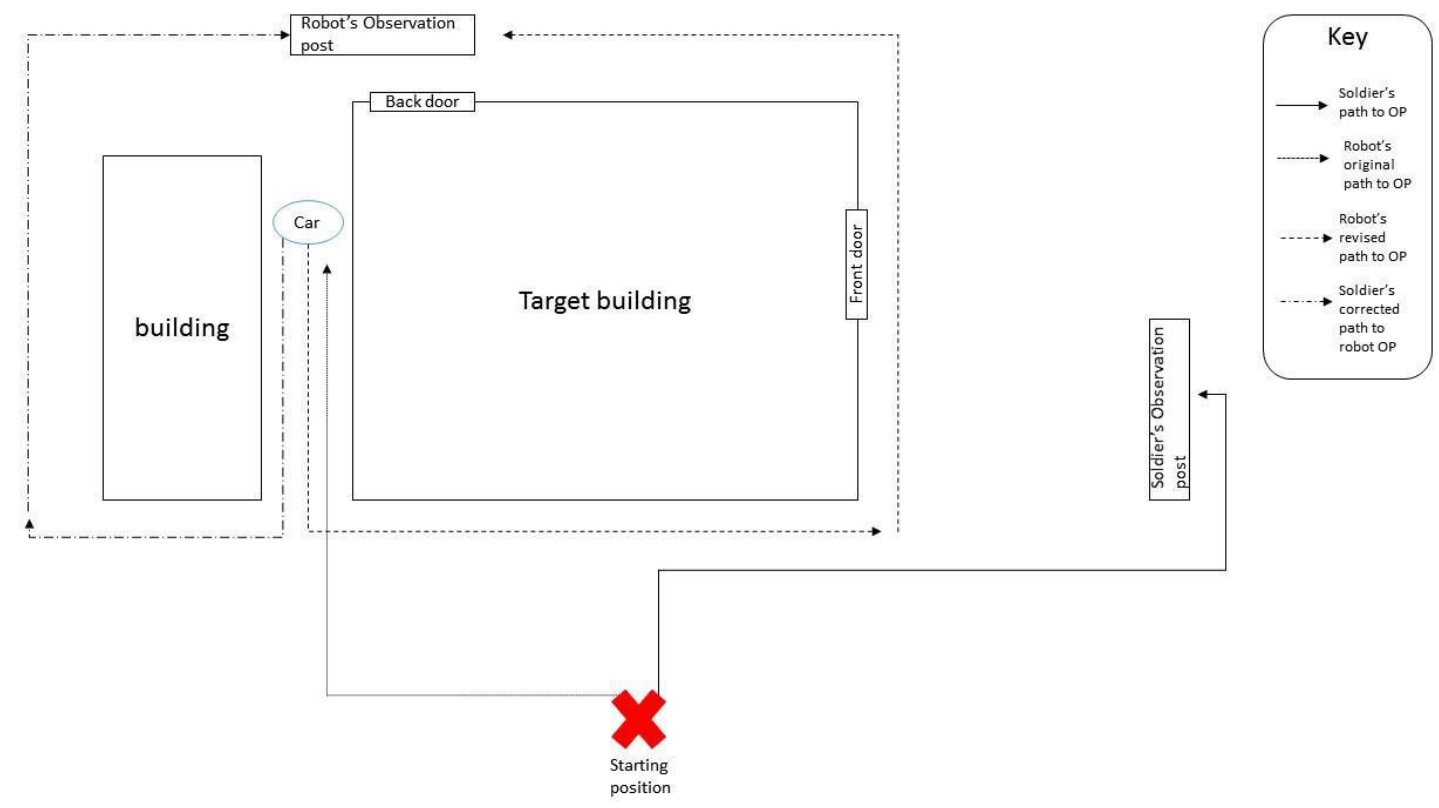

Figure 5.The Soldier realizes that the robot's revised path is undesirable and makes a correction. Figure includes a graphical representation of the Soldier's revision to the robot's path.

With the previous alert, the robot provided the Soldier with additional visuospatial processing and made a projection about relevant SA information on behalf of the Soldier's unique point of view. Information exchanges that include additional visuospatial processing by the robot, may, thus, help to foster higher levels of awareness regarding the robot, which Soldiers can use to intervene in robot tasks when necessary. This ability can allow the robot to become an active participant in the Soldier's SA regarding robot and its operating environment. 


\section{$\underline{\text { Significance for military contexts }}$}

Although, in the near term, the cognitive capabilities and perceptual skills of robots will not match their human counterparts, this does not mean that robots cannot serve as valuable assets in future SR teams. In fact, a study by Schuster (2013) showed that even unreliable information provided by a robot can help a human develop SA when the ability of the human to do so unaided is poor. Further, U.S. ARMY doctrine specifies that "Every soldier is a sensor" on the battlefield (United States, 2008). As such, human teammates will expect robots to contribute to operator SA by sharing information with human teammates in an effective, proactive way (ARL, 2012; Schuster, Keebler, Zuniga, \& Jentsch, 2012). For the near term, there is a need to understand how information sharing capabilities of robots can be leveraged to provide useful gains in operator SA.

For instance, Groom and Nass (2007) suggested that a unique advantage of robots is that they can perceive information through multiple viewpoints, because sensors can be made distinct from the robot itself. A robot can rely on reference points like global positioning systems (GPS), to localize their physical location in an environment, or make use of CCTV cameras to understand the physical features of their environment. A robot supplying a Soldier with additional VPT processing when exchanging information is a complementary means to leverage the unique capabilities of human and robots, by taking advantage of a robot's ability to easily perceive and process multiple points of view and 
of the Soldier's ability to utilize shared information to develop situation awareness regarding the robot, and intervene when needed.

Applying VPT to robot-to-human information exchanges may be an efficient way to allow robots to contribute to human SA regarding the robot, which will be used to aid the robot when continuous monitoring is unavailable, and allow the military to field robots in the near term, without necessitating full automation or continuous monitoring of the robot.

\section{$\underline{\text { Purpose }}$}

The purpose of this study was to test the effects of robot-to-human information exchanges on the development of human SA regarding a robot, and human assistance provided to a robot under differing levels of VPT. It was suggested that SA regarding a robot would be necessary to provide assistance to a robot when needed. Further, it was proposed that adding differing levels of VPT information to robot-to-human information exchanges could support differing levels of SA regarding the robot. Figure 6 shows a conceptual model of the relationships between levels of VPT and levels of SA regarding the robot. 


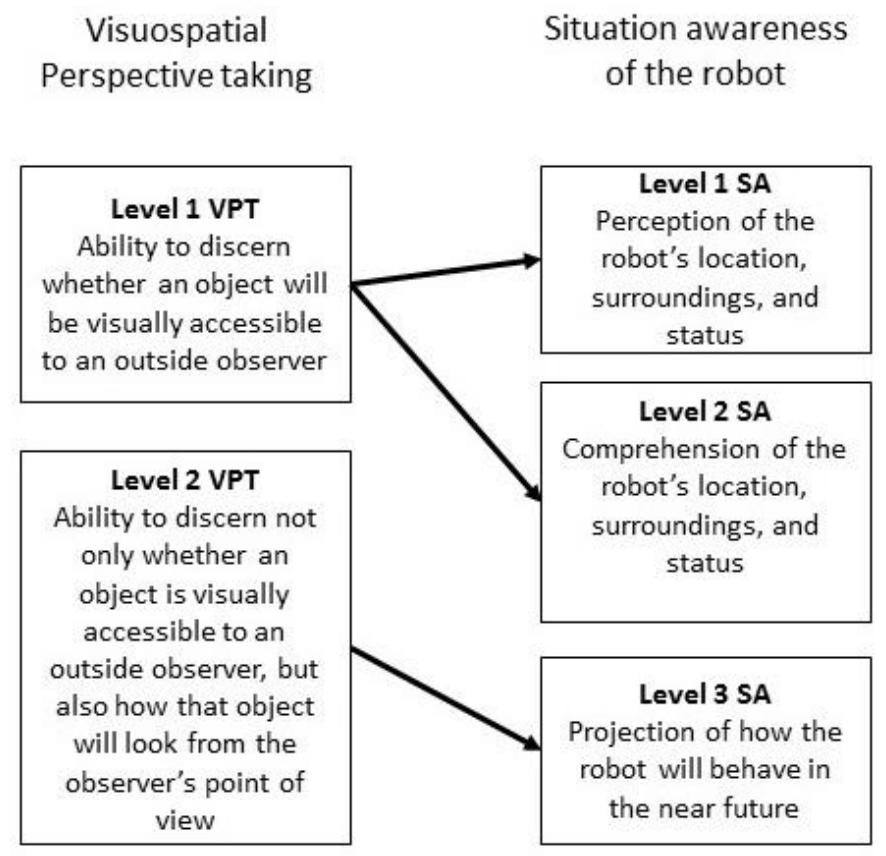

Figure 6. Conceptual mapping of levels of VPT to levels of SA regarding the robot.

The study was concerned with a human's development of SA and the ensuing ability to provide assistance to a robot when necessary, as a result of robot information sharing behaviors in a human — robot team task. The results of this study will be used to inform the development of near future robot capabilities, including information sharing behaviors in robots intended to be fielded alongside Soldiers in battlefield environments. 


\section{CHAPTER TWO: LITERATURE REVIEW}

\section{$\underline{\text { Situation awareness and human assistance provided to a robot }}$}

In order for a Soldier to provide necessary assistance to a robotic teammate, the Soldier will need to maintain SA regarding the robot and its operating environment. To illustrate, recall the screen the back door (STBD) vignette beginning on page 12 of the Introduction. In this scenario, the Soldier would not have known that an intervention to the robot's path was needed, unless he/she had awareness regarding how the robot was going to behave in the near future (i.e., that the robot's projected path, depicted in Figure 4, was going to place the robot at risk of being detected).

Military doctrine concerning the STBD mission implies that team members should avoid detection, as capture could jeopardize the success of the mission (United States, 2012). As such, in the STBD vignette (see Figure 5), when the Soldier makes the intervention that aides the robot in avoiding detection, the Soldier performs an action that is essential for the team to successfully perform the mission, and is predicated on the Soldier maintaining SA regarding the robot (i.e., the perception of the robot's location, surroundings, and status, Level 1; the comprehension of their meaning, Level 2; and the projection of how the robot would behave in the near future, Level 3, Yanco \& Drury, 2004). For STBD missions, the performance of the team is thus highly dependent on the Soldier maintaining SA regarding the robot. 
For many other human—robot interaction tasks, maintaining SA regarding the robot is a vital part of successful team performance as well. However, prior research has shown that maintaining SA regarding the robot is very difficult for human operators to do (Burke, Murphy, Coovert, \& Riddle, 2004; Casper \& Murphy, 2003; Groom \& Nass, 2007; Murphy \& Burke, 2005). In their analysis of human—robot interaction in urban search and rescue (SAR) tasks, Yanco and Drury (2004) reported that $30 \%$ of each rescue run was spent acquiring SA (including SA regarding the robot) at the exclusion of all other activities. Often, Robot operators expressed uncertainty concerning where their robot was located in the environment relative to other environmental landmarks (i.e., Level 1 and Level 2 SA regarding the robot).

On the other hand, prior research has shown that when operators are able to maintain SA regarding the robot, the performance of the human-robot team is improved. For instance, in a longitudinal study investigating multiple years of a humanrobot interaction in urban SAR competition, Yanco and Drury (2007) noted several instances in which a team's best performance, across multiple years of competition, coincided with a change in the presentation of remote video of the robot, on the humanrobot interaction display (e.g., increases in the overall size of the display, removal of occlusions to video coming from the robot). For each team, SA regarding the robot (especially levels 2 and 3) was primarily maintained via remote video that was presented through this display. Changes in the display likely led to better SA regarding the robot's location in the environment, its surroundings, and how the robot's surroundings affected 
its ability to move through the environment, which helped operators to better navigate through the competition's course. Other studies that have addressed SA-focused improvements to video displays and interaction devices (e.g., map overlays, vehicle status windows) for unmanned aerial vehicles (Drury, Richer, Rackliffee, \& Goodrich, 2006), unmanned ground vehicles (Drury, Keyes, \& Yanco, 2007), and automated road vehicles (Scholtz, Antonishek, \& Young, 2004) have shown similar improvements in human—robot team performance as well.

In multiple field studies investigating the role of SA on human-robot interaction team performance for urban SAR tasks, Murphy and Burke (2005; Burke \& Murphy, 2004) reported that rescue teams with high situation awareness were nine times more likely to successfully use teleoperated robots to find victims among rubble, than teams with low situation awareness. Teams with high SA were rated $26 \%$ higher on subjective measures of performance than teams with low SA. Human-robot interaction performance was also improved when team members aided each other in building SA regarding how the robot was situated in the environment. Together, these findings support the notion that SA regarding the robot, including its location, surroundings, and how the robot will move through the environment is an important factor for successful performance in other types of human—robot team tasks, in addition to STBD.

In these studies (Murphy \& Burke, 2005; Burke \& Murphy, 2004; Yanco \& Drury, 2007), superior performance of the human — robot team was contingent upon the 
robot operator maintaining good awareness of the robot, its relative position in the environment, and how that position could affect the robot's ability to continue maneuvering through the environment. For operators, maintaining good SA regarding the robot lead to effectively providing navigation support to the robot, which primarily came in the form of maneuvering the robot around rubble, debris, and other obstacles. These actions were essential for the team to successfully complete the SAR tasks. For future Soldier-robot team tasks, like STBD, SA regarding the robot will be similarly necessary, as the Soldier will need to be aware of the robot's location, surroundings, and how the robot is projected to behave, in order to provide assistance to the robot and ensure the successful completion of the team's mission.

These findings provided the basis for the first experimental Hypothesis of the current study (see Figure 7).

Hypothesis H1: Higher levels of SA regarding the robot will be positively associated with superior human assistance provided to the robot. 


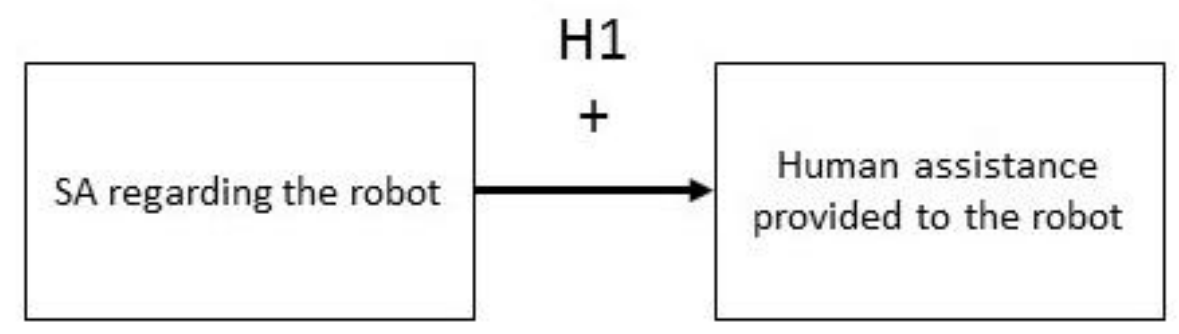

Figure 7. Graphical representation of Hypothesis H1.

If situation awareness regarding the robot is needed to facilitate operator assistance in robot tasks, it is necessary to explore processes that foster situation awareness regarding the robot. Fong, Kunz, Hiatt, and Bugajska (2006) stated that information exchanged between humans and robots can foster situation awareness in a human-robot team:

Humans and robots coordinate their actions through dialogue. This helps contextual and situation awareness to be maintained across the team (p. 41).

Although this suggestion was not empirically tested, other researchers have similarly supported the notion that exchanging information between team members can provide clues about what other members are aware of in their environment and in the completion of their tasks (Salmon, Stanton, Walker, \& Jenkins, 2009). The next section is dedicated to exploring the literature on how information sharing in human-human teams supports team member SA, with the goal to apply insights to future Soldier-robot teaming. 


\section{Robot-to-human information sharing}

Information sharing between robots and humans may be the key for developing awareness regarding the robot that is required to assist the robot when needed. This section is dedicated to reviewing a body of research on human-human teams, which links patterns of information sharing to the development if SA within a team, as well as team performance. Specifically, information sharing in the form of alerts and updates, are mechanisms that foster situation awareness among team members. Team members also provide supplemental contextual information to shared information; and, added contextual information is associated with superior team performance and the development of SA within teams.

To illustrate, communication, marked by altering, updating, and providing information about the state of the team's task completion, has been associated with the emergence of SA among team members. A study by Parush et al. (2011) observed surgical teams performing ten open-heart surgeries to investigate the communicative processes by which SA is developed and maintained within surgical teams. The researchers focused on speech acts, in which situation-related information was shared among the team. Results revealed that the largest proportion of speech within the team was spent announcing information (i.e., reporting on a value, state, or action taken) or, directing, instructing, and/or requesting members to report actions regarding the state of the equipment and procedures undertaken by individual members. These results suggest 
that the majority of speech within these teams was intended to build awareness of the state of the patient and processes undertaken by individual team members. Similar results have been found for teams working in energy distribution systems (Salmon, Stanton, Walker, \& Jenkins, 2009) as well. Salmon et al. noted that when team members share information in the form of status updates and alerts, SA-relevant information is exchanged between members, and SA among the team is updated and maintained.

Team members also transfer information that provides a more "complete picture" of the situation. In a study examining communications of anesthesiologists during neurosurgery operations, Johannesen et al. (1994) described that team members helped to build the "complete picture" by providing extra information that contributed context for the responses given to information queries; more so than would be provided by simply answering the request for information alone. The researchers also described that team members kept each other aware of the relevant influences to the process of task completion, and this action allowed other members to make more accurate predictions about future states of task completion. Similar results have been found for studies investigating teams performing in novel/unfamiliar environments as well (Marks, Zaccaro, \& Mathieu, 2000). These researchers noted that team members who engage in higher quality communications (i.e., communications that transferred more valuable information to team members) outperformed teams who engaged in lower quality communication. In general, these findings suggest that high-performing teams transfer more meaningful information by providing context for their teammates. 
Information transfers that provide additional context have been found to be important for the development of SA as well. A study by Hazlehurst, McMullen, and Gorman (2007) investigated how situation awareness becomes an emergent property within a team. Specifically, the researchers were interested in how coordination within a cardiac surgical team controlled the surgical system (i.e., patient, team members, and equipment) and made predictable the state of the system as the team completed the surgical task. The researchers analyzed communication exchanges within the team and noted specifically, that status, alert, and explanation exchanges fostered understanding of the current state of the system. Importantly, they stated that explanation exchanges helped members reduce uncertainty about the state of the system and the processes that led to the current state. An example of an explanation information exchange is given below.

Surgeon: How much you got in?

Perfusionist: 400

Surgeon: Let's go to 750. She's got a good arrest, ventricle's empty. You can see it on the echo, that empty ventricle, it's not distending (p. 548).

The researchers described that the surgeon could have simply stated that the perfusionist needed to increase the dosage to 750 , but the added explanation provided extra context for the information shared. Similar results have also been found for studies investigating anaesthesia teams (Fioratou, Flin, Glavin, \& Patey, 2010). 
Overall, these results have shown that information sharing in the form of alerts, and updates, in teams plays an important role in team performance and in the development of situation awareness. The results of the studies on information sharing and situation awareness support the notion that information sharing, for example, in the form of updates and alerts, can help team members develop awareness of the activities of other members and the state of the team's completion of the task. Because sharing information in the form of status updates helps team members build SA, it is reasonable to forecast that robot-to-human information exchanges in the form of status updates will be associated with SA regarding the robot as well. Similarly, because performance in human-human teams is improved by team members sharing information in the form of status updates to other team members, it is reasonable to suggest that robots that share information in the form of status updates with human team members will also facilitate performance in human-robot teams. Based on the results of the studies summarized, the following was given for the second and third Hypotheses of the current study (see Figure 8).

Hypothesis H2: Robot-to-human information exchanges will be positively associated with situation awareness regarding the robot, such that more exchanges will be associated with better $S A$ regarding the robot than fewer exchanges ${ }^{2}$.

\footnotetext{
${ }^{2}$ It should be noted that robot-to-human information exchanges will likely have an upper bound on the number that is beneficial. After that upper threshold is reached, it is possible that robot-to-human information exchanges could hinder the development of SA and performance.
} 
Hypothesis H3: Robot-to-human information exchanges will be positively associated with human assistance provided to the robot, such that more exchanges will be associated with better human assistance provided to the robot than fewer exchanges. .

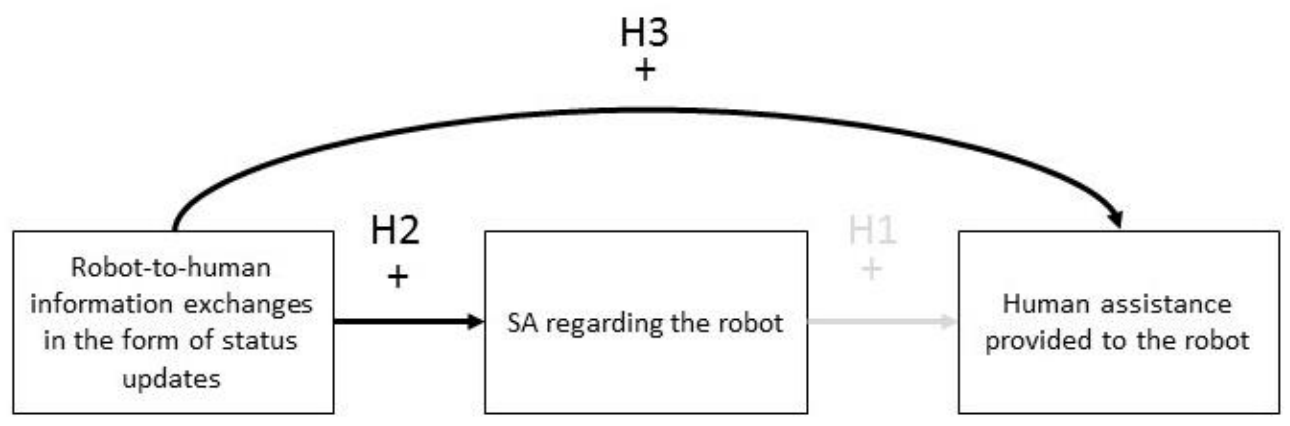

Figure 8. Graphical representation of Hypotheses $\mathrm{H} 1, \mathrm{H} 2$, and $\mathrm{H} 3$, including the mediating effect of SA regarding the robot on the relationship between robot-to-human information exchanges and human assistance provided to the robot.

The relationships outlined in Hypotheses $\mathrm{H} 2$ and $\mathrm{H} 3$ should also be influenced by the amount of context added to information that is shared by the robot to the human. The results summarized above show that high-performing human-human teams not only transfer more information in the form of updates and alerts, they also transfer context rich information, which is associated with the development of SA among the team and team performance. As a result, it is reasonable to expect that for human-robot teams, transfers of context rich information updates will yield even better results for team performance and the development of SA, than information transfers alone. The presence of additional context should influence the relationship between robot-to-human information exchanges 
and human assistance provided to the robot, as well as robot-to-human information exchanges and SA regarding the robot; such that, information exchanges should support higher levels of SA regarding the robot under conditions of high context. Similarly, robot-to-human information exchanges should support better human assistance provided to the robot under conditions of high information context.

For future Soldier-robot teams, the robot's integration of situational views (i.e., visuospatial perspective taking) will provide additional context when the robot shares information with the Soldier; specifically, spatial context that will be useful in maintaining SA regarding the robot and its operating environment, when the ability to continuously monitor the robot is removed. The next section will be dedicated to reviewing how adding visuospatial perspective context to robot-to-human information exchanges should influence the development of SA regarding the robot, as well as human assistance provided to the robot.

\section{$\underline{\text { Visuospatial perspective taking }}$}

Information about visuospatial perspective taking (VPT) will serve as appropriate additional context that a robot can add to robot-to-human information exchanges because VPT is associated with spatial skills, which have been linked to the development of SA in individuals (Carretta, Perry, \& Ree, 1996; Endsley \& Bolstad, 1994), as well as

performance in human - robot teams (Fincannon, 2013). Further, VPT context can minimize the mental effort needed for a human to build an understanding of the robot's 
surroundings and the robot's location in the operating environment (Fincannon, 2013; Shelton \& McNamara, 2004). As a result, higher levels of VPT information added to robot-to-human information exchanges should foster higher levels of SA regarding the robot (see Figure 6), as well as better human assistance provided to the robot.

In general, VPT refers to the ability to imagine how a scene would appear visually from someone else's literal view of the environment ${ }^{3}$. (Schwarzkopf, Schilbach, Vogeley, \& Timmermans, 2014). VPT is primarily a visuospatial skill in the domain of spatial orientation (i.e., spatial skills that involve the ability to make egocentric transformations); and involves the ability "to imagine the result of changing one's egocentric frame of reference with respect to the environment" (Hegarty \& Waller, 2004, pp. 187-188). When the robot applies VPT context to information transfers, it is performing additional spatial processing for the human, which should anchor information according to the human's unique view of the environment, as opposed to the robot's view of the environment (i.e., “There is a building at your 11 o'clock" vs. "There is a building on my left"). Doing so is consistent with prior research which suggested that when individuals are provided with spatial information, anchored in their view of the environment, they are better able to learn and understand spatial layouts (Shelton \& McNamara, 2004).

\footnotetext{
${ }^{3}$ VPT is unlike other conceptualizations of Perspective Taking outside the psychological literature (e.g., narratology, philosophy, cognitive science, and neuroscience). For a review of perspective taking, see Streater, Elias, Bockleman Morrow, \& Fiore (2011).
} 
As a result, applying VPT information to robot-to-human information exchanges should improve the human's perception of the robot as it is situated in its environment and surroundings. This perception will serve as the foundation for developing higher levels of SA and subsequent interactions with the robot, including providing assistance to the robot.

\section{Spatial skills and situation awareness}

Extra spatial processing by the robot should help in the development of SA regarding the robot because space and spatial relationships are often critical components of maintaining and building SA during complex tasks. The perception of space and spatial relationships plays an important role in how individuals build SA of their operating environment (Endsley, 1995a). In many operational contexts, space and spatial relations are important "elements" that comprise the components of the task or environment that operators need to perceive in order to acquire higher levels of SA.

In addition to its aspect as a frequent "element" of SA, spatial information is highly useful for determining exactly which aspects of the environment are important for SA (p. 38).

In many contexts, including human - robot interaction, SA is highly spatial in nature. When piloting aircraft, for instance, spatial awareness of the location of the aircraft in relation to the location of other aircraft, is frequently of concern. 
For STBD tasks, spatial perception of elements in the environment, including the location of the robot team member, will be essential components of SA as well. In the STBD vignette beginning on page 12 of the Introduction, for the Soldier to successfully re-route the robot's path around the building (to prevent the robot from being detected), the Soldier needs to have a fairly accurate understanding of the robot's current position and projected position in the environment, as well as the spatial relationship between the robot and other key features of the environment (e.g., the main door of the building, for instance). From a theoretical standpoint, if spatial information is an important element of SA for a particular task, or task environment, then the ability to understand spatial information should be inherently linked to the ability to develop SA. Studies investigating the relationship between spatial skills and the development of SA have supported this notion.

Specifically, spatial skills have been linked to individual differences in the ability to develop situation awareness in studies by Carretta, Perry, and Ree (1996), as well as Endsley and Bolstad (1994). The study by Endsley and Bolstad investigated the degree to which individual differences on a variety of factors theorized to be related to the development of SA (e.g., memory, spatial ability, attention) were indeed related to an individual's ability to develop SA. The researchers found that spatial ability, including a measure of spatial orientation (the spatial factor associated with visuospatial perspective taking), was significantly correlated with objective measures of SA. Better performance on the spatial orientation measure was significantly associated with higher SA. 
A similar study by Carretta, Perry, and Ree (1996) investigated predictors of SA ability in F-15 pilots. In this study, Air Force pilots were rated by subject matter experts on situation awareness skills as well as on several personal traits related to situation awareness, including spatial skills and flying experience. Results showed that flying experience was the most important variable for predicting pilot SA ratings. However, when holding flying experience constant, spatial ability was also predictive of SA ability; though other personal traits theorized to be associated with SA (e.g., psychomotor skills, personality) were not.

\section{Spatial skills and performance in human-robot teams}

Spatial understanding is highly relevant in the context of human-robot team performance as well. A meta-analysis by Fincannon (2013), revealed a main effect for visuo-spatial ability in predicting performance with unmanned vehicles (UVs) across several performance dimensions, including identifying and localizing targets in remote environments, as well as navigating robotic vehicles through remote environments. This is important to note because Fincannon's definition of navigation performance described having awareness of the UV and its environment, which is very similar to the formal definition of SA (i.e., the perception of elements in the environment within a volume of time and space, Level $1 \mathrm{SA}$; the comprehension of their meaning, Level $2 \mathrm{SA}$; and the projection of their status in the near future, Level 3 SA, Endsley, 1995a). 
Fincannon's definition of navigation performance included awareness of the UVs location in the environment (Level $1 \mathrm{SA}$ ), as well as how those features are relevant (Level $2 \mathrm{SA}$ ) for developing a projection for moving the UV through the environment (Level 3 SA).

While navigation includes processes that are related to the localization of components of a remote environment (e.g., UV, targets, future objective, route obstructions), it goes further by requiring the operator to use that understanding of a UV in relation to a variety of other elements in the environment to develop a future-oriented strategy for moving through that environment (Fincannon, 2013, p17).

As such, navigation performance for operating UVs in remote environments likely requires elements of SA regarding the UV (i.e., robot), which lends additional support for the linkage between spatial ability, the development of SA, and subsequent humanrobot interaction performance (including assisting the robot in its navigation through the environment).

In a similar paper summarizing experiments investigating the role of spatial ability and human-robot interaction performance, Chen (2011) described that high spatial ability is linked to superior performance for a variety of human-robot interaction tasks, including teleoperating robotic medical devices, searching for targets using unmanned robotic vehicles, and replanning routes for unmanned aerial vehicles. Chen also described 
an experiment in which participants were asked to locate targets in their surrounding environment and simultaneously utilize a robot to help locate targets in several remote environments. Results revealed that participants with high spatial ability outperformed participants with low spatial ability in the percentage of targets detected in both the surrounding environment, and across all the remote environments. Together, these studies provide evidence for the importance of spatial understanding in supporting human - robot team performance.

For STBD tasks, spatial information about the robot and other features of its operating environment (e.g., buildings, doors, obstacles) will be important for the completion of the mission. If the robot is able to perform some spatial processing for the human, by adding VPT context to information exchanges in the form of status updates, then the robot is making it easier for the human to develop SA regarding the robot. Sharing information that enhances spatial understanding will, ultimately, be more meaningful for the human and for the completion of the team's tasks.

The findings given above support the idea that spatial understanding is associated with situation awareness and performance in human-robot interaction tasks, such that those with higher spatial abilities tend to outperform those with lower spatial abilities on measures of situation awareness, as well as performance measures in human-robot interaction tasks. Spatial relationships between the robot and features of its operating environment will constitute "elements" important for developing SA regarding the robot 
in tasks like STBD. Because VPT is a spatial ability related to those associated with the ability to develop SA, as well as team performance in human-robot interaction tasks, it is fair to suggest that VPT context is appropriate for supporting situation awareness regarding the robot and human performance as measured by the ability to provide assistance to the robot in tasks like STBD. As greater spatial ability is associated with better SA, information exchanges that include higher levels of spatial information (VPT) should lead to better SA regarding the robot. Since greater spatial ability is also associated with superior performance in human-robot interaction tasks, information exchanges that include higher levels of spatial information (VPT) should lead to better performance in providing assistance to the robot as well. As such, the following was given for Hypotheses H4 and H5 (see Figure 9).

Hypothesis H4: Robot-to-human information exchanges that include higher levels of VPT context will lead to higher levels of SA regarding the robot than robot-to-human information exchanges that include lower levels of VPT context.

More specifically, robot-to-human information exchanges that include Level 2 VPT context will be associated with higher levels of SA regarding the robot (i.e., level 3SA), than robot-to-human information exchanges that include Level 1 VPT context (i.e., levels 1 and 2 SA). 
Hypothesis H5: Robot-to-human information exchanges that include higher levels of VPT context will lead to better human assistance provided to the robot, than robot-to-human information exchanges that include lower levels of VPT context.

More specifically, robot-to-human information exchanges that include Level 2

VPT context will be associated with higher quality of human assistance provided to the robot, than robot-to-human information exchanges that include Level 1 VPT context.

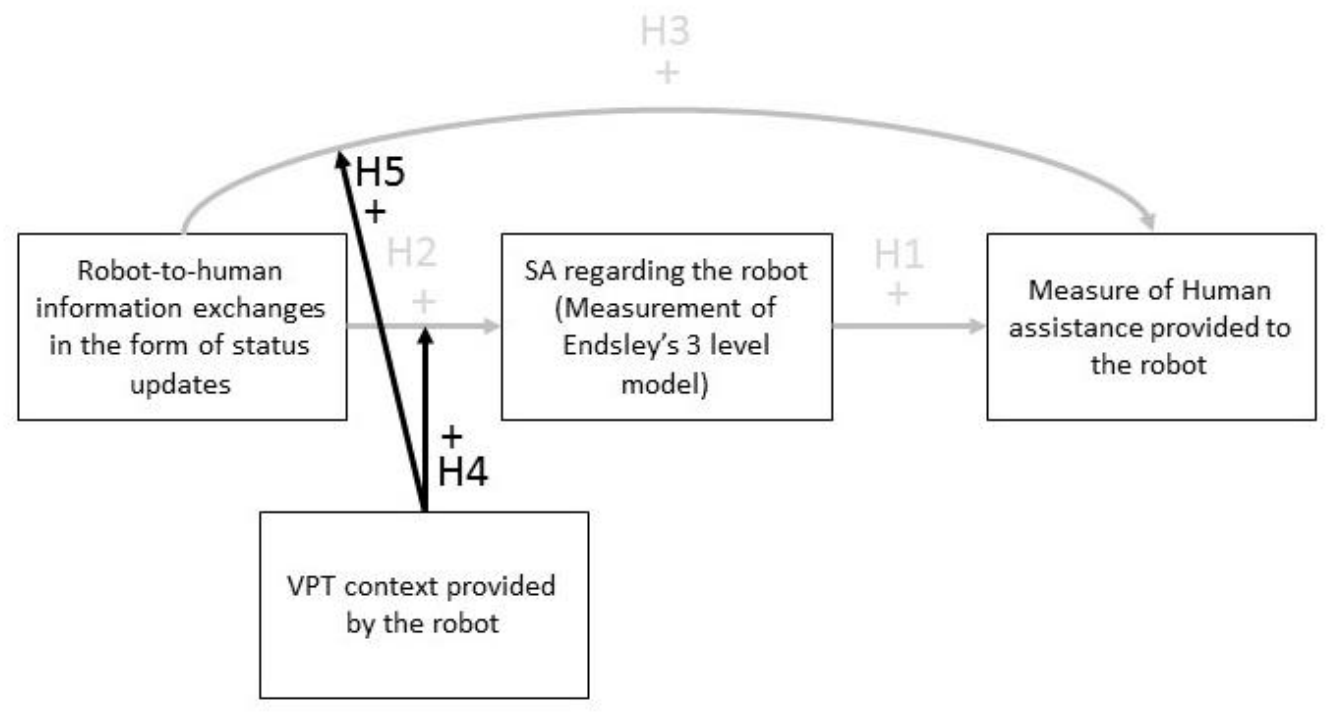

Figure 9. Graphical representation of Hypotheses H1, H2, H3, H4, and H5. 


\section{Summary information}

The following summary presents: the study model (Figure 10), a listing of the hypotheses (Table 1), a table of constructs of interest (Table 2) and a discussion of potential confounds to the study.

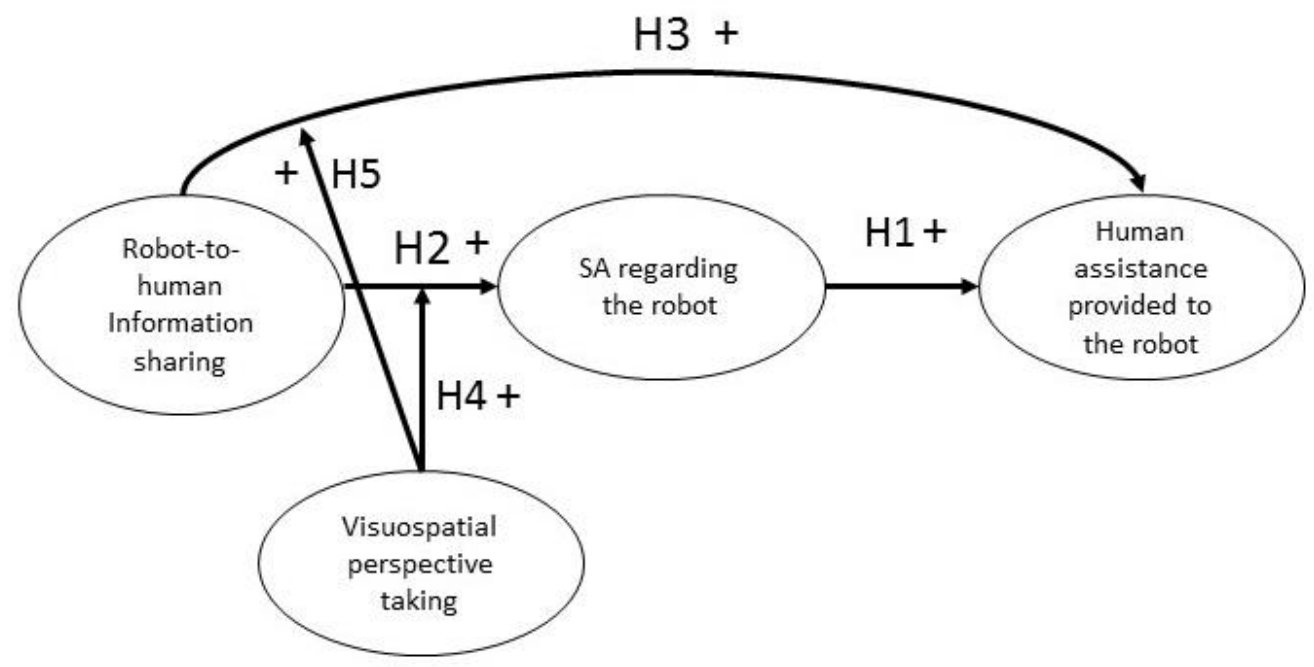

Figure 10. Study model. 
Table 1. Summary of hypotheses

Statement of hypotheses

H1: Higher levels of SA regarding the robot will be positively associated with superior human assistance provided to the robot.

H2: Robot-to-human information exchanges will be positively associated with situation awareness regarding the robot, such that more exchanges should be associated with better SA regarding the robot than fewer exchanges.

H3: Robot-to-human information exchanges will be positively associated with human assistance provided to the robot, such that more exchanges should be associated with better human assistance provided to the robot than fewer exchanges.

H4: Robot-to-human information exchanges that include higher levels of VPT context will lead to higher levels of SA regarding the robot than robot-to-human information exchanges that include lower levels of VPT context

More specifically, robot-to-human information exchanges that include Level 2 VPT context will be associated with higher levels of SA regarding the robot (i.e., level 3SA), than robot-to-human information exchanges that include Level 1 VPT context (i.e., levels 1 and 2 SA).

H5: Robot-to-human information exchanges that include higher levels of VPT context will lead to better human assistance provided to the robot, than robot-tohuman information exchanges that include lower levels of VPT context.

More specifically, robot-to-human information exchanges that include Level 2 VPT context will be associated with higher quality of human assistance provided to the robot, than robot-to-human information exchanges that include Level 1 VPT context. 
Table 2. Constructs of interest

\begin{tabular}{|c|c|c|c|c|c|}
\hline Construct Name & $\begin{array}{l}\text { Operationalized } \\
\text { Variable }\end{array}$ & $\begin{array}{l}\text { Affected } \\
\text { Variable(s) }\end{array}$ & Impact & $\begin{array}{l}\text { Proposed } \\
\text { Analysis }\end{array}$ & References \\
\hline Information sharing & & $\begin{array}{l}\text { Human assistance } \\
\text { provided to the } \\
\text { robot }\end{array}$ & Positive & $\begin{array}{l}\text { ANOVA } \\
\text { Planned } \\
\text { comparisons }\end{array}$ & $\begin{array}{l}\text { Johannesen, Cook, \& Woods (1994) } \\
\text { Marks, Zaccaro, \& Mathieu (2000) }\end{array}$ \\
\hline $\begin{array}{l}\text { Visuospatial } \\
\text { perspective taking }\end{array}$ & $\begin{array}{l}\text { Visuospatial } \\
\text { perspective taking } \\
\text { context provided by } \\
\text { the robot }\end{array}$ & $\begin{array}{l}\text { SA regarding the } \\
\text { robot } \\
\text { Human assistance } \\
\text { provided to the } \\
\text { robot }\end{array}$ & $\begin{array}{l}\text { Positive } \\
\text { Positive }\end{array}$ & $\begin{array}{l}\text { ANOVAs } \\
\text { Planned } \\
\text { comparisons }\end{array}$ & $\begin{array}{l}\text { Carretta, Perry, \& Ree (1996) } \\
\text { Endsley \& Bolstad, (1994) } \\
\text { Chen (2011) } \\
\text { Chen, Barnes, \& Qu (2010) } \\
\text { Fincannon (2013) }\end{array}$ \\
\hline $\begin{array}{l}\text { Operator } \\
\text { intervention in robot } \\
\text { tasks }\end{array}$ & $\begin{array}{l}\text { Human assistance } \\
\text { provided to the robot }\end{array}$ & $\mathrm{n} / \mathrm{a}$ & $\begin{array}{l}\text { Dependent } \\
\text { variable }\end{array}$ & & Fong, Thorpe, \& Baur (2003) \\
\hline
\end{tabular}




\section{Potential confounds}

Endsley's 3-Level model of SA postulates that there are several individual and task factors that affect one's ability to develop SA. Because Endsley's theory of SA is based upon information processing theory (Endsley, 1995a), there are several information processing mechanisms and individual difference factors that play vital roles in one's ability to develop SA, including working memory, mental models, and prior experience. Workload is a relevant task factor that affects the development of SA as well. Further, high ability on visuospatial perspective taking could nullify the benefit of adding VPT context to robot-to-human exchanges in the development of SA regarding the robot and human assistance provided to the robot (see Table 7).

\section{Individual differences in working memory}

One's working memory ability plays an important role in one's ability to develop SA. Working memory plays a role in the direction of attentional resources by influencing how well one is able to hold, in memory, previously perceived information, as well as active goals. As such, working memory can also function as a limiting factor in the development of SA. Once perception is achieved, working memory provides the foundation for higher levels of SA (i.e., Levels 2 and 3).

New information must be combined with existing knowledge and a composite picture of the situation developed (Level 2 SA). Projections of future status (Level 
$3 \mathrm{SA}$ ) and subsequent decisions as to appropriate courses of action must occur in working memory as well (Endsley, 1995a, p. 43).

For this study, individual differences in working memory were measured and scores were evaluated for suitability to serve as covariates in the analyses of the study hypotheses. Visuospatial working memory was measured using a computerized version of the Corsi Block Tapping Task (Corsi, 1972)

\section{Mental models}

Mental models are also important for the development of SA, as they provide the prior knowledge of the task and team that direct perception. Mental models include knowledge of features of the environment/task that are relevant for perception (Level 1 SA). They also include knowledge of how relevant features are integrated to derive meaning from the situation (Level 2 SA), and can serve as a system model for projecting future states (Level 3 SA, Endsley, 1995a, p.44). Task mental models will provide the foundation for understanding which features of the environment are relevant for successful mission completion. Humans will also need to hold mental models of the robot, as knowledge of how the robot operates, including its capabilities and limitations, will be important for making projections about how the robot will behave in the environment. For instance, if the human's mental model of the robot includes knowledge that the robot cannot maneuver through rocky terrain, then this information dictates that rocky terrain will be a relevant element of the environment that will need to be perceived 
in order to direct the robot's movement around said terrain. For the current study, participants were trained on features, capabilities, and details of the experimental task and robotic teammate. Participants' retention of this information was evaluated using a Training retention questionnaire.

\section{Prior knowledge of robots and robotics}

One's prior knowledge of robots and the robotics domain is also a relevant individual difference variable, as prior knowledge influences one's mental models of the capabilities and limitations of robots, and mental models provide a foundation for SA. In this study, prior knowledge of robots was measured and scores were evaluated for suitability to be covaried in subsequent analyses. Prior knowledge of robotics and robotics technologies was measured using a biographical information measure.

\section{Mental workload}

Workload is a factor that is associated with one's ability to develop SA as well. Endsley (1995a) described that perceived workload represents a form of socialpsychological stressor. When situational stress is high, SA is primarily affected through a narrowing of attentional resources, which limits the number of environmental factors in which one is able to attend. The result of this attentional narrowing is to place priority on certain elements of the environment over others, which can lead to reduced perception and degrade Level 1 SA. Although workload can negatively affect one's ability to develop SA, Endsley (1995a) has stated that high workload does not guarantee poor SA. 
The following may exist...High SA with high workload: The operator is working hard, but is successful in achieving an accurate and complete picture of the situation (p. 53).

For this study, a moderate level of workload was imposed using a dual-task paradigm via a change detection task (Abich, Reinerman-Jones, and Taylor, 2011; Abich, 2013). Workload was measured using the NASA-TLX (Hart \& Staveland, 1988).

\section{Individual differences in perspective taking ability}

In the psychological literature, VPT is conceptualized as a visuospatial skill associated with spatial orientation skills. Hegarty and Waller (2004) described that VPT skill involves the ability to make

Egocentric spatial transformations in which one's egocentric reference frame changes with respect to the environment, but the relation between object-based and environmental frames of reference does not change (Thurstone, 1950) (p.176).

Making these spatial transformations requires difficult mental processing (Shelton \& McNamara, 2004). As such, the degree to which a robot, in a human—robot team, could make these egocentric transformations for the human should be beneficial, as effortful spatial processing will be offloaded to the robot. However, the benefit of the robot making these spatial transformations may be nullified if the ability of the human to make egocentric transformations themselves is already high. Individual differences in 
VPT ability was measured and scores were evaluated for their suitability to be used as covariates in the analyses of the study hypotheses. Individual differences in participants' visuospatial perspective taking ability was measured using the Perspective Taking/Spatial Orientation Test (Hegarty \& Waller, 2004). See table 3 for a summary of potential confounding variables. 
Table 3. Table of potential confounding variables

\begin{tabular}{|c|c|c|c|c|c|c|}
\hline Variable & Construct & Definition & Reference & $\begin{array}{l}\text { Affected } \\
\text { Variable }\end{array}$ & Reference & $\begin{array}{l}\text { Method for } \\
\text { Control }\end{array}$ \\
\hline Workload & $\begin{array}{l}\text { Mental } \\
\text { Workload }\end{array}$ & $\begin{array}{l}\text { A measureable quantity of information processing } \\
\text { demands placed on an individual by a task (p. 77). }\end{array}$ & $\begin{array}{l}\text { Sanders \& } \\
\text { McCormick } \\
(1993)\end{array}$ & $\begin{array}{l}\text { SA regarding } \\
\text { the robot }\end{array}$ & $\begin{array}{l}\text { Endsley } \\
\text { (1995) }\end{array}$ & $\begin{array}{l}\text { Impose/ } \\
\text { Control }\end{array}$ \\
\hline $\begin{array}{l}\text { Working } \\
\text { memory ability }\end{array}$ & $\begin{array}{l}\text { Working } \\
\text { memory }\end{array}$ & $\begin{array}{l}\text { A brain system that provides temporary storage and } \\
\text { manipulation of the information necessary for such } \\
\text { complex cognitive tasks as language comprehension, } \\
\text { learning, and reasoning (p. 556). }\end{array}$ & Baddeley (1992) & $\begin{array}{l}\text { SA regarding } \\
\text { the robot }\end{array}$ & $\begin{array}{l}\text { Endsley } \\
(1995) \\
\text { Endsley } \\
(1988)\end{array}$ & Covary \\
\hline $\begin{array}{l}\text { Mental model } \\
\text { of the robot } \\
\text { Mental model } \\
\text { of the task }\end{array}$ & $\begin{array}{l}\text { Mental } \\
\text { models }\end{array}$ & $\begin{array}{l}\text { Mechanisms whereby humans are able to generate } \\
\text { descriptions of system purpose and form, explanation } \\
\text { of system functioning and observed system states, } \\
\text { and predictions of future states (p.7). }\end{array}$ & $\begin{array}{l}\text { Rouse \& Morris } \\
(1986)\end{array}$ & $\begin{array}{l}\text { SA regarding } \\
\text { the robot }\end{array}$ & $\begin{array}{l}\text { Endsley } \\
(1995) \\
\text { Wickens \& } \\
\text { Hollands } \\
(1984)\end{array}$ & Train \\
\hline $\begin{array}{l}\text { Prior robotics } \\
\text { experience }\end{array}$ & Experience & $\begin{array}{l}\text { Mental models are natural evolving systems that tend } \\
\text { to change with experience (p.2) }\end{array}$ & $\begin{array}{l}\text { Ososky et al., } \\
(2012)\end{array}$ & Mental Models & $\begin{array}{l}\text { Norman } \\
(1983)\end{array}$ & Covary \\
\hline VPT ability & $\begin{array}{l}\text { Individual } \\
\text { differences in } \\
\text { spatial ability }\end{array}$ & $\begin{array}{l}\text { Ability to make spatial transformations in which } \\
\text { one's egocentric frame of reference changes with } \\
\text { respect to the environment (p.176). }\end{array}$ & $\begin{array}{l}\text { Hegarty \& } \\
\text { Waller (2004) }\end{array}$ & $\begin{array}{l}\text { Robot-to- } \\
\text { human } \\
\text { information } \\
\text { exchanges }\end{array}$ & $\begin{array}{l}\text { Shelton \& } \\
\text { McNamara } \\
(2004)\end{array}$ & Covary \\
\hline
\end{tabular}




\section{CHAPTER THREE: METHOD}

\section{Participants}

After data screening and reduction (see Results section), and to help control for the influence of participant biological sex in the analyses ${ }^{4}, 56$ male participants with ages ranging from 18 to $29(M=18.89, S D=3.412)$ were included in this study. Participants were recruited from the University of Central Florida's Psychology Department's undergraduate research pool using Sona Systems research recruitment software. Participants volunteering for this study were required to be enrolled in an undergraduate class in the Psychology department. Participants were screened for age to ensure that they were at least 18 years of age or older. Students demonstrated eligibility to participate in the study (i.e., age, and course registration) by signing up for an account with Sona Systems and filling out a pre-screening questionnaire. In accordance with University policy, which dictates that students demonstrate an adequate level of English language comprehension, researchers anticipated that participants would be able to read and write in English.

\footnotetext{
analyses.

${ }^{4}$ See Appendix I for more detail concerning the influence of participant biological sex in the
} 


\section{Design}

A within-subjects design was used for this study. All participants completed 4 experimental missions associated with each of the study conditions (described below). The order of completion of the four missions/study conditions was assigned to participants utilizing two Latin squares. Figure 11 shows a graphical representation of the two Latin Squares that were used for this study. Stratified random assignment was used to assign participants to order conditions and to ensure that a comparable number of participants completed each order designated by the Latin squares.

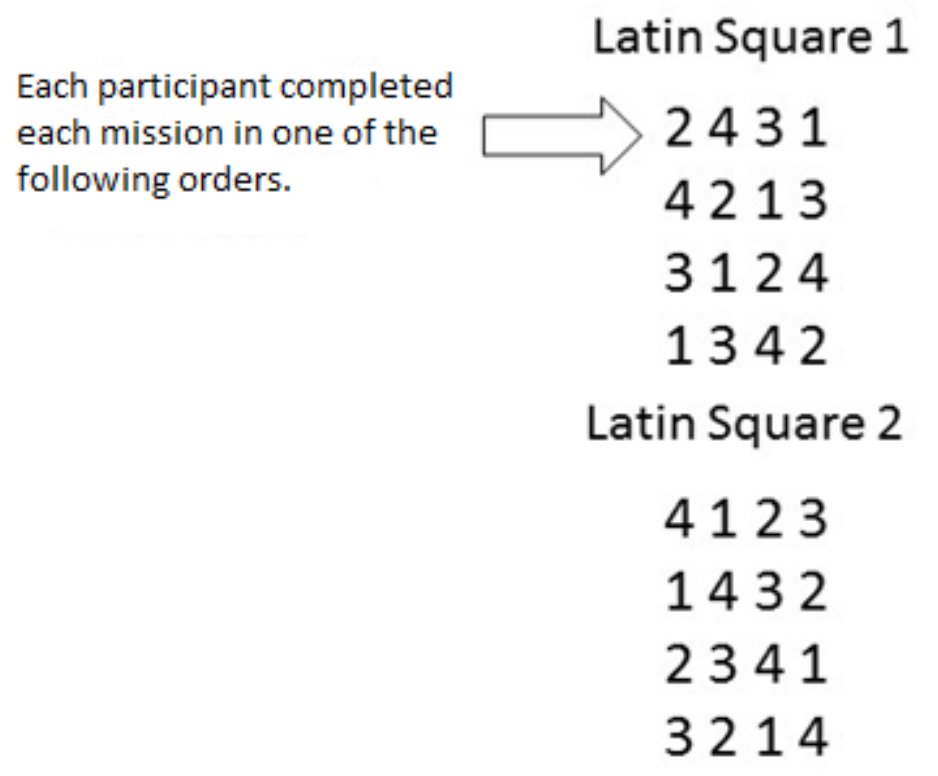

Figure 11. Graphical representation of two Latin squares that were used to determine the order in which participants completed each mission associated with each study condition. 


\section{Experimental task}

The experimental scenario designed for this study was a reconnaissance and surveillance task completed by a human-robot team. Participants were informed that their mission was to help in the testing and development of an experimental unmanned ground robot capable of being commanded to carry out tasks without continuous human supervision. The participants were informed that their mission was to work together with a simulated robot on a reconnaissance and surveillance mission by completing several objectives. Participants were told that their role would be to (a) play the role of a Soldier in a simulated Soldier-robot team, (b) provide surveillance of an area of interest and make note of changes to that area (i.e., the change detection task), (c) use updates from the robot (i.e., waypoint and traveling status updates, described below) to keep track of the robot's movement and answer questions regarding the robot's movement and location (i.e, SA regarding the robot questions, described below), (d) provide re-routing information that helps the robot to avoid sensitive areas, in the event that the robot veered off course (i.e., providing assistance to the robot, described below), and (e) pay attention to how quickly the robot is completing its objectives. In this team, the robot was responsible for navigating a waypoint path through the environment, and periodically sharing updates with the Soldier regarding the buildings located at each waypoint, as well as other status information concerning its navigation through the environment. 
Throughout each mission, the waypoint movement of the robot was scripted and the participant was not responsible for maneuvering the robot.

During the course of each mission, the robot periodically shared status updates with the Soldier concerning its movement through the environment. Specifically, once the robot reached each waypoint, it made a waypoint status update back to the Soldier. This report included information that identified the buildings located on either side of the robot (e.g., "There is a church on one side and a park on the other"), as well as other status information (i.e., the robot's status as stopped, the estimated time to reach the end of its route, and its estimated percentage of the planned route the robot had completed). Figure 12 presents an example of a status update that was given to the Soldier by the robot, each time the robot reached a waypoint along its path. 


\title{
Waypoint 1
}

\section{Status: Stopped/Scanning}

\author{
Time to target: $3 \mathrm{~m}$ 00s \\ Percent complete: $\mathbf{1 5 \%}$
}

\section{Intel: There is a church on one side and a residence on the other.}

Figure 12. Example of a robot-to-human information exchange in the form of a waypoint status update.

The robot also periodically exchanged information, in the form of traveling status updates, concerning the direction of its movement through the environment. (e.g., "Heading North towards the next waypoint"). Figure 13 provides an example of a traveling status update. The participants were responsible for using the information contained in the status updates (both waypoint and traveling) to keep track of the robot's location in the environment, answer SA-related questions regarding the robot, and to provide assistance to the robot, by re-routing the robot back to the planned path through the environment, in the event that the robot deterred from its planned route. 


\section{Status: Maneuvering}

\section{Heading: North}

\section{Towards next waypoint}

Figure 13. Example of a robot-to-human information exchange in the form of a traveling status update, given while the robot was maneuvering between waypoints.

\section{Change detection task}

While receiving status updates from the robot, the participant, playing the role of the Soldier, was asked to engage in a dual task in the form of a change detection task. Participants were asked to monitor an overhead map of an urban environment on which various icons were displayed. Figure 14 provides an example of the overhead map and icons displayed to the participants. The overhead map which displayed the change detection icons was not an overhead view of the robot's environment. 
In the change detection task, the icons could exhibit three possible changes.

Changes included disappearing from the screen, appearing on the screen, or moving across the screen. Several virtual buttons were present on the participants' screen. Participants used the "Appeared", "Disappeared", and "Movement" virtual buttons to indicate the type of change event that occurred. Participants were not asked to identify which specific icon or set of icons changed when a change event occurred.

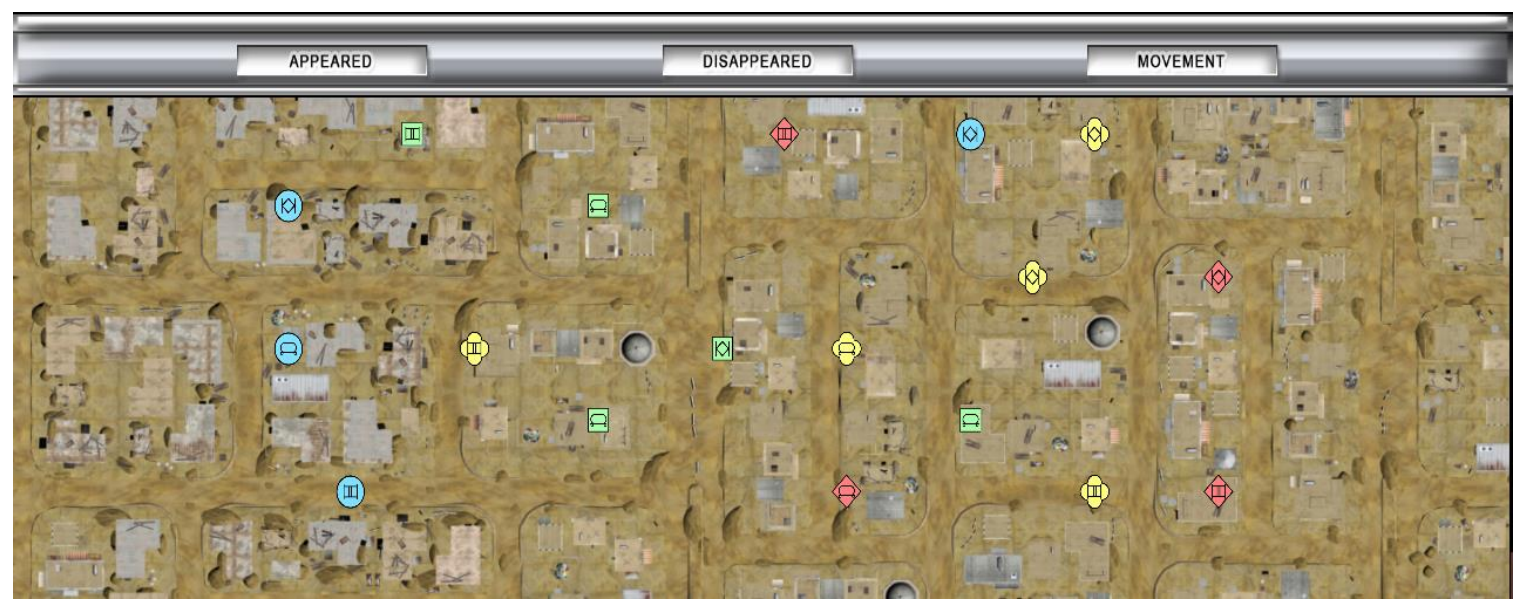

Figure 14. Example of the overhead map and icons displayed for the change detection task.

At the start of each mission, 18 icons were present on the screen. In addition, each change event included 2 icons changing at the same time (i.e. medium signal saliency), with both icons exhibiting the same type of change. The change detection task presented an event rate of 12 changes per minute (i.e., medium event rate), which exhibited a change, on average, once every 5 seconds. The timing of each change event was randomized by the simulation software used to administer the study. The event rate, 
signal saliency, and number of icons present at the start of each mission were used to impose a medium level of workload for the experimental task. See Abich, ReinermanJones, and Taylor (2011) and Abich (2013) for a detailed description of signal saliency, event rate, and various task load manipulations that can potentially be induced using the change detection task.

Also, the change detection task was used to provide an active task for the participants to complete while the robot was navigating through the virtual environment and sending status updates to the participant. Performance on this task was used to examine the effects of the various experimental manipulations on dual task performance.

\section{$\underline{\text { Study conditions }}$}

All participants completed four simulated missions associated with the following robot-to-human information exchange conditions.

\section{Waypoint status updates (7 updates) condition}

Under the Waypoint status updates (7 updates) condition, the participant, playing the role of the Soldier, received status updates when the robot reached each waypoint. That is, the Soldier received status updates that reported on the buildings located on either side of the robot, the robot's status as stopped, its estimated time to reach the end of its route, and its estimated percentage of completion of its planned route (see Figure 12). Also, for this condition, participants received waypoint status updates throughout the 
course of the mission 7 times. For the remainder of this document, this condition will be referred to as the 7UP condition.

\section{Waypoint status updates (13 updates) condition}

Under the waypoint status updates (13 updates) condition, the robot stopped at roughly twice as many waypoints as the Waypoints status updates (7 updates) condition. This resulted in the participant receiving almost twice as many robot-to-human information exchanges in this condition as in the Waypoint status updates ( 7 updates) condition. Additionally, the number of robot-to-human information exchanges received in the Waypoint status updates (13 updates) condition was intended to be approximately equal to the number of robot-to-human information exchanges received in the Traveling + waypoints status updates conditions (described below). For the remainder of this document, this condition will be referred to as the 13UP condition.

\section{Traveling + waypoint status updates conditions with Level 1 VPT condition}

In this condition, participants received traveling status updates that reported on the direction that the robot was heading between waypoints in addition to the waypoint status updates similar to those in the conditions described above. This resulted in participants receiving approximately 13 robot-to-human information exchanges that were presented as a combination of waypoint and traveling status updates. In addition, the traveling status updates were presented in reference to Level 1 VPT context. Such that, 
the traveling status updates included information concerning the direction the robot was traveling between waypoints in global-relative, cardinal, intermediate, and/or combination point directions (e.g., heading North/South/Southwest/South-Southwest). For this condition, a compass rose that depicted the four cardinal directions (i.e., North, South, East, and West) was presented on an overhead map of the environment (see materials section). For the remainder of this document, this condition will be referred to as the 13UPLevel1 condition.

Traveling + waypoint status updates conditions with Level 2 VPT condition

Under this condition, participants received traveling status updates that reported on the direction that the robot was heading between waypoints in addition to the waypoint status updates similar to those in the conditions described above. This also resulted in participants receiving 13 robot-to-human information exchanges. However, in this condition, the traveling status updates were presented in reference to Level 2 VPT context. Level 2 VPT context presented the direction that the robot was traveling between waypoints in terms relative to the Soldier's position in the environment (e.g., heading 6 o'clock, West) in addition to global-relative, directions (see Figure 15).

Clock face directions were chosen to denote Level 2 VPT context because Level 2 VPT represents the ability to infer how objects appear to be situated in an environment in relation to an outside observer's view of those objects, as well as the outside observer's position in reference to those objects. Clock face directions communicate relative bearing 
in reference to the direction the navigator in a team/crew/ship is facing. As such, Level 2 (i.e., clock face) information provided by the robot, is Soldier-relative as opposed to global or robot-relative, and allows the robot to provide information to the Soldier that is specific to how the Soldier would perceive environmental information. In addition, clock face coding is a method for communicating relative bearing/navigation information that is standard in military aviation, seafaring, and land navigation contexts (United States; 2009). Thus, using clock face directions maintains consistency with terminology that is familiar to the people intended to be interacting with military robots in the near future.

For this condition, the compass rose presented on the overhead map of the environment, depicted clock position directions (i.e., o'clocks, 12, 3, 6, and 9). For the remainder of this document, this condition will be referred to as the 13UPLevel2 condition. Table 4 presents conceptual descriptions of the study conditions and associated study missions.

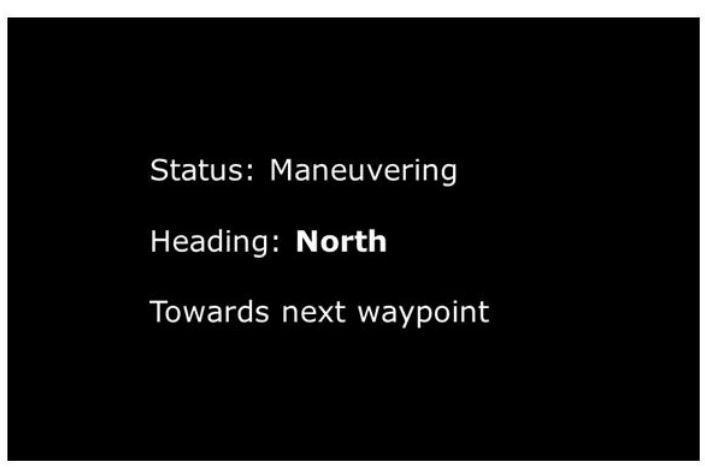

Level 1 VPT context

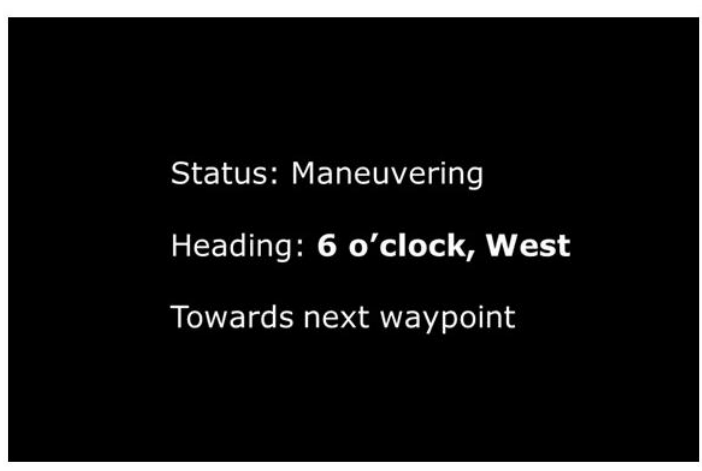

Level 2 VPT context

Figure 15. Examples of Level 1 and Level 2 VPT context added to traveling updates. 
Table 4. Table of within-subjects conditions. All participants completed conditions numbered 1, 2, 3, and 4.

\begin{tabular}{|c|c|c|c|c|}
\hline $\begin{array}{c}\text { Mission } \\
\#\end{array}$ & Conditions & $\begin{array}{l}\text { Description of } \\
\text { mission }\end{array}$ & $\begin{array}{l}\text { Description of Robot-to- } \\
\text { human information } \\
\text { exchanges }\end{array}$ & $\begin{array}{l}\# \text { of } \\
\text { exchanges } \\
\text { /updates }\end{array}$ \\
\hline 1 & $\begin{array}{l}7 \mathrm{UP} \\
\text { condition }\end{array}$ & $\begin{array}{l}\text { Participant receives } \\
\text { an update when the } \\
\text { robot reaches a } \\
\text { waypoint. }\end{array}$ & $\begin{array}{l}\text { Updates report on the } \\
\text { buildings located on either } \\
\text { side of the robot, the } \\
\text { robot's status as stopped, its } \\
\text { estimated time to reach the } \\
\text { end of its route, and its } \\
\text { estimated percentage of the } \\
\text { planned route it has already } \\
\text { completed. }\end{array}$ & 7 \\
\hline 2 & $\begin{array}{l}\text { 13UP } \\
\text { condition }\end{array}$ & $\begin{array}{l}\text { Participant receives } \\
\text { an update when the } \\
\text { robot reaches a } \\
\text { waypoint. Robot } \\
\text { will stop at } \\
\text { approximately } \\
\text { twice as many } \\
\text { waypoints as in } \\
\text { 7UP condition. }\end{array}$ & $\begin{array}{l}\text { Updates report on the } \\
\text { buildings located on either } \\
\text { side of the robot, the } \\
\text { robot's status as stopped, its } \\
\text { estimated time to reach the } \\
\text { end of its route, and its } \\
\text { estimated percentage of the } \\
\text { planned route it has already } \\
\text { completed. }\end{array}$ & 13 \\
\hline 3 & $\begin{array}{l}\text { 13UP } \\
\text { Level1 } \\
\text { condition }\end{array}$ & $\begin{array}{l}\text { Participant receives } \\
\text { updates from the } \\
\text { robot while it is } \\
\text { traveling between } \\
\text { waypoints in } \\
\text { addition to } \\
\text { receiving updates } \\
\text { when the robot } \\
\text { reaches each } \\
\text { waypoint. }\end{array}$ & $\begin{array}{l}\text { Updates report the direction } \\
\text { of the robot's movement at } \\
\text { Level } 1 \text { VPT (e.g., } \\
\text { "Heading East towards the } \\
\text { next waypoint") in addition } \\
\underline{\text { to }} \text { the waypoint status } \\
\text { updates. }\end{array}$ & 13 \\
\hline
\end{tabular}




\begin{tabular}{|c|c|c|c|c|}
\hline & Conditions & $\begin{array}{l}\text { Description of } \\
\text { mission }\end{array}$ & $\begin{array}{l}\text { Description of Robot-to- } \\
\text { human information } \\
\text { exchanges }\end{array}$ & $\begin{array}{l}\text { \# of } \\
\text { exchanges } \\
\text { /updates }\end{array}$ \\
\hline 4 & $\begin{array}{l}\text { 13UP } \\
\text { Level2 } \\
\text { condition }\end{array}$ & $\begin{array}{l}\text { Participant receives } \\
\text { updates from the } \\
\text { robot while it is } \\
\text { traveling between } \\
\text { waypoints in } \\
\text { addition to } \\
\text { receiving updates } \\
\text { when the robot } \\
\text { reaches each } \\
\text { waypoint. }\end{array}$ & $\begin{array}{l}\text { Updates report the direction } \\
\text { of the robot's movement at } \\
\text { Level } 2 \text { VPT (e.g., } \\
\text { "Heading East, at } 3 \text { o'clock } \\
\text { towards the next } \\
\text { waypoint") in addition to } \\
\text { the waypoint status updates. }\end{array}$ & $\begin{array}{l}\text { Approx. } \\
13\end{array}$ \\
\hline
\end{tabular}

\section{$\underline{\text { Materials }}$}

Simulation testbed and experimental apparatus

This study utilized the Mixed-Initiative Experimental Testbed (MIX, Barber, Leontyev, Sun, Davis, Nicholson, and Chen, 2008) as the simulation platform for the completion of experimental tasks as well as experimental data collection. The MIX testbed is an open-source virtual simulation research environment that allows for the study of human — robot interaction between semi-autonomous unmanned vehicles and human participants in collaborative virtual mission scenarios. MIX is primarily comprised of two components. These include the operator control unit (OCU), and the unmanned systems (i.e., unmanned aerial, ground, and/or surface vehicles) simulator (USSIM). The OCU is capable of presenting multiple sources of information from the simulated unmanned vehicles, including information like communications, first person camera views, and overhead maps of the environment. 
This study utilized the OCU to present robot-to-human information exchanges in the form of status updates to the participants and to support the change detection task. Figure 16 provides a screen shot of the OCU used for this study. Robot-to-human information exchanges were presented to participants in the upper right-hand portion of the screen, the change detection task was completed in the lower half of the screen, and the upper left-hand portion of the screen contained a mission timer also referred to as a countdown clock. Please note that participants did not have access to the robot's real-time location on the OCU or the robot's first person view of the environment. 


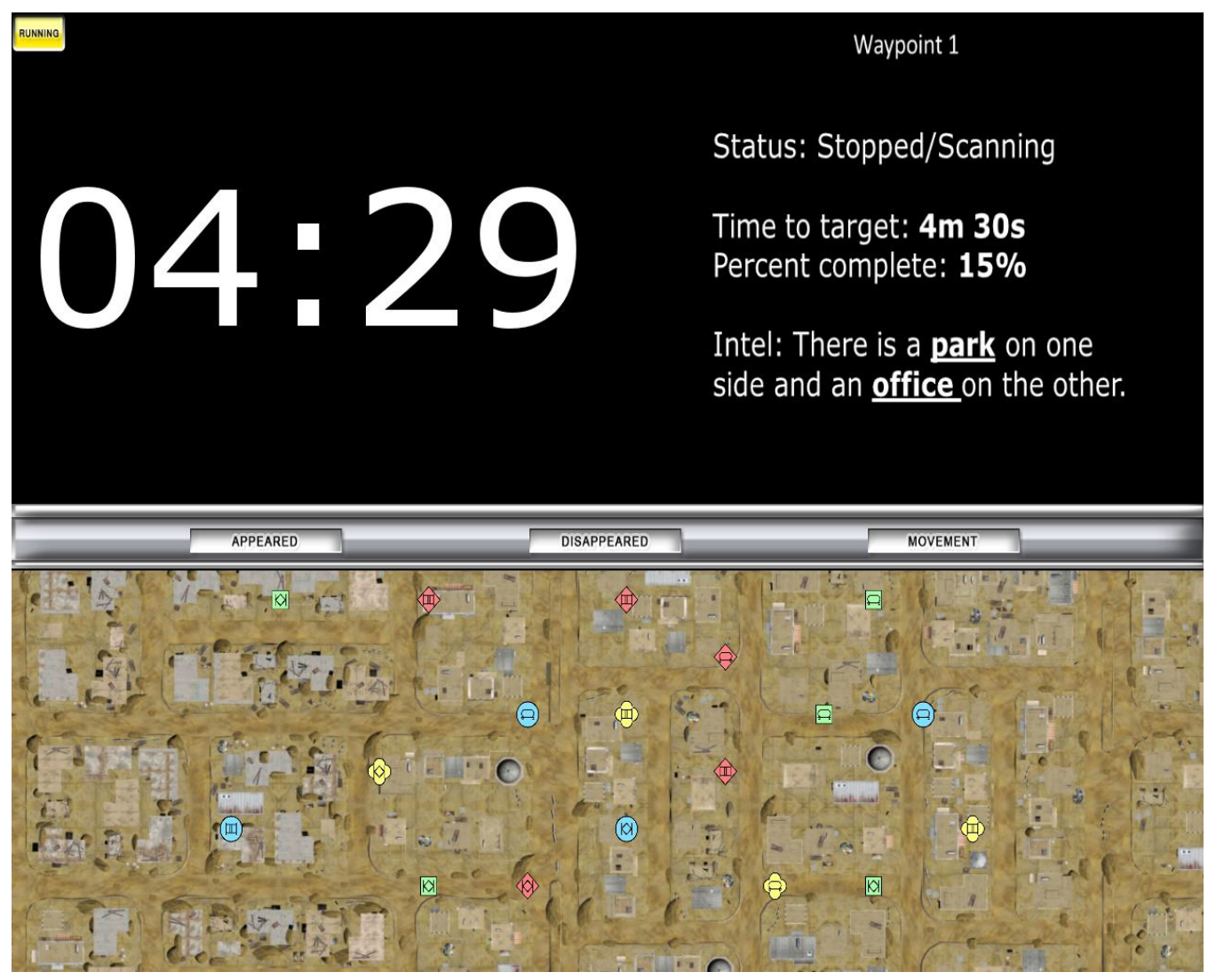

Figure 16. Screen shot of the OCU in the MIX simulation testbed. Note, the overhead map displayed at the bottom portion of the OCU was not an overhead view of the robot's environment or the robot's waypoint route.

Figure 17 contains a photograph of the physical experimental apparatus. The apparatus consisted of a workstation that included a 22 inch computer monitor, keyboard, mouse, and headphones, in addition to a participant binder, which contained maps for the rerouting task, a pen, a pencil, and an 8 1/2 x 14 inch, legal size binder which was used to display the overhead mission maps to the participants. The legal size binder displayed to the right of the participants' computer monitor (see Figure 17) displayed the overhead 
maps of the robot's environment and its planned waypoint route through the environment. Three identical workstations were created for this study.

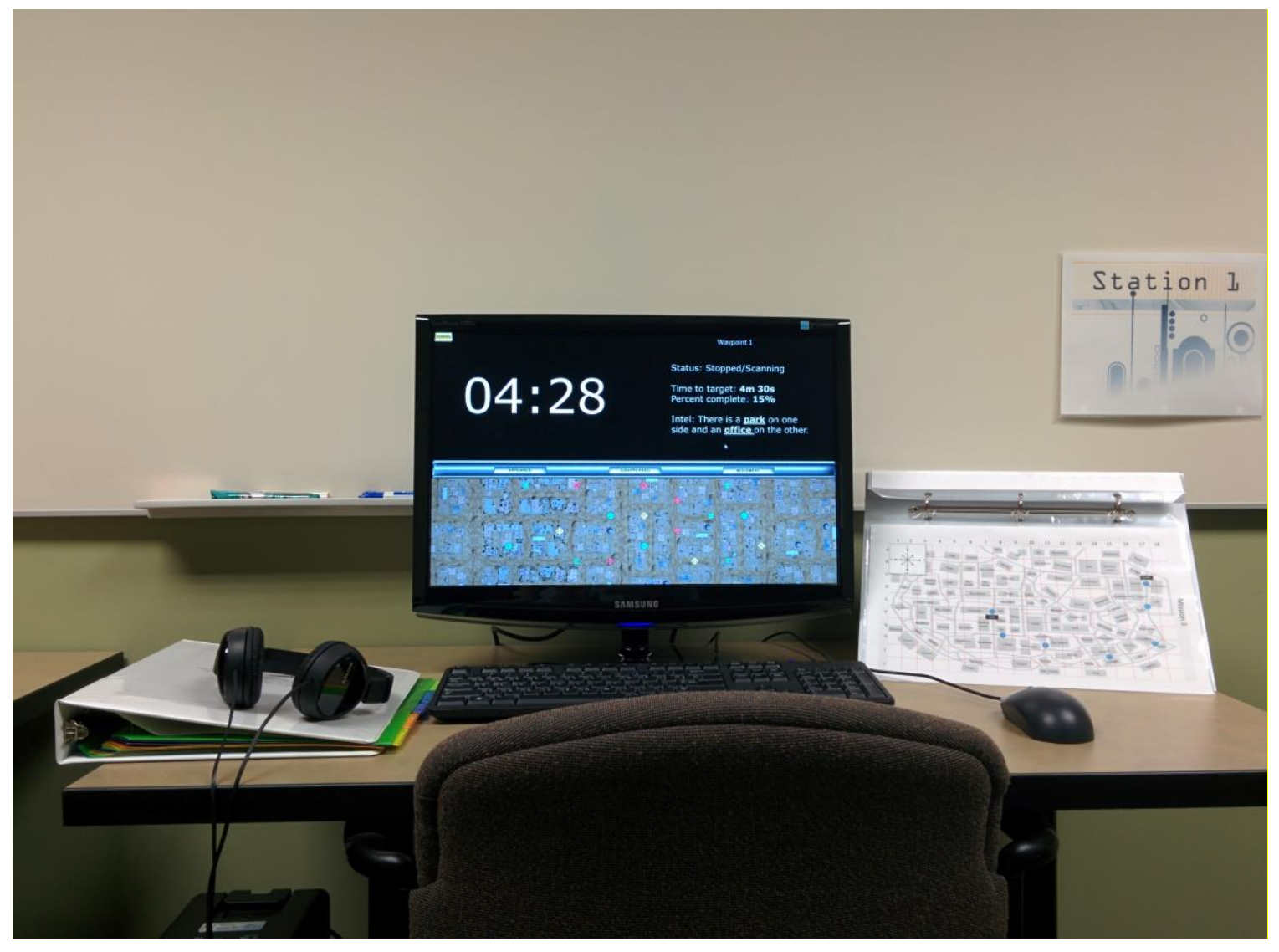

Figure 17. Photograph of the physical experimental apparatus.

\section{Training}

Training materials were presented in a combination of Microsoft Office

PowerPoint $® 2013$ (PowerPoint) and the MIX testbed. Training materials presented in 
Power Point are included in the appendices of this document. A part-task training paradigm was used to present participants with opportunities to practice performing individual elements of the experimental task in isolation. Training concluded with participants completing 3 training missions that aggregated all the individual task elements together. The training phase of the study took approximately 90 minutes to complete.

PowerPoint training materials consisted of a narrated slide presentation that included information on the background story and setting of the research study, as well information on how to complete the change detection task, and the experimental tasks. Participants began the training session by viewing the portion of the PowerPoint presentation that provided background information about the surveillance and reconnaissance mission, the simulated Soldier-robot team's objectives for the study, the tasks the participant, playing the role of the Soldier, would be asked to complete and information about the change detection task. Training slides regarding the change detection task included information about the types of changes that icons could exhibit as well as how to indicate the type of change event that occurred.

Once participants finished viewing this portion of the training presentation, participants were directed to the MIX simulation to complete a practice trial of the change detection task. The practice trial of the change detection task lasted approximately 1 minute and 30 seconds and contained approximately 15 change events with an average 
event rate of one change every 5 seconds and a signal saliency of 2 icons changing per individual change event (See discussion of the change detection task).

Once the change detection practice trial was complete, participants were directed back to PowerPoint to view a series of slides that presented the details of the experimental tasks. Specifically, participants were provided information regarding the types of status updates they would receive from the robot (i.e., waypoint updates and the two types of traveling updates), as well as information regarding the countdown clock presented in the OCU.

After presenting this information, the PowerPoint presentation then reviewed elements of the two types of compass roses that would be used in this study. Specifically, the training included information about the various types of directions that can be presented on a compass rose, including cardinal (i.e., North, South, East, and West), intermediate (e.g., Northeast, Southwest), and Combination point directions (e.g., NorthNortheast, South-Southwest, etc.). A few slides were then dedicated to practicing and reviewing the information about the compass rose (see Figure 18). Participants were then asked to point to the area on the compass rose that corresponded to the given direction. The correct answer was then highlighted in the presentation. 


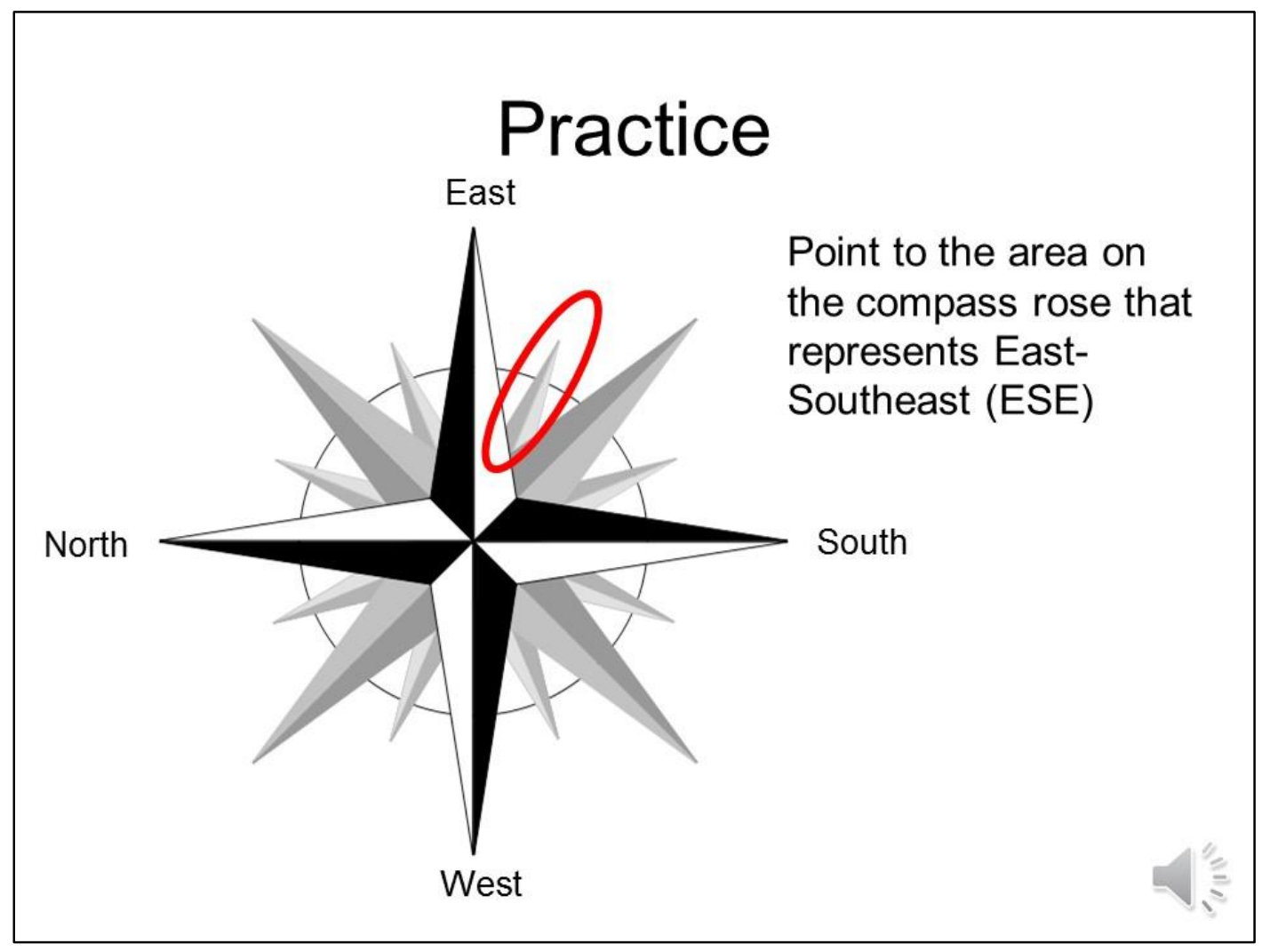

Figure 18. Example of training materials for the compass rose.

Next, participants were provided with information regarding how to interpret global relative directions, as clock face directions (see Figure 19). This information was intended to help participants understand that no matter the orientation of the cardinal directions, clock face directions are always relative to themselves with 12 o'clock always representing straight ahead. The slide depicted in Figure 19 was animated in order to convey this information. 


\section{Clock face}

- Imagine that you are in the center of a clock face.

West

- Straight ahead is 12 o'clock.

-6 = behind you

$-3=$ to your right

-9 = to your left

- Independent of cardinal directions.

- Aid in interpreting map orientation when North is not "straight ahead".

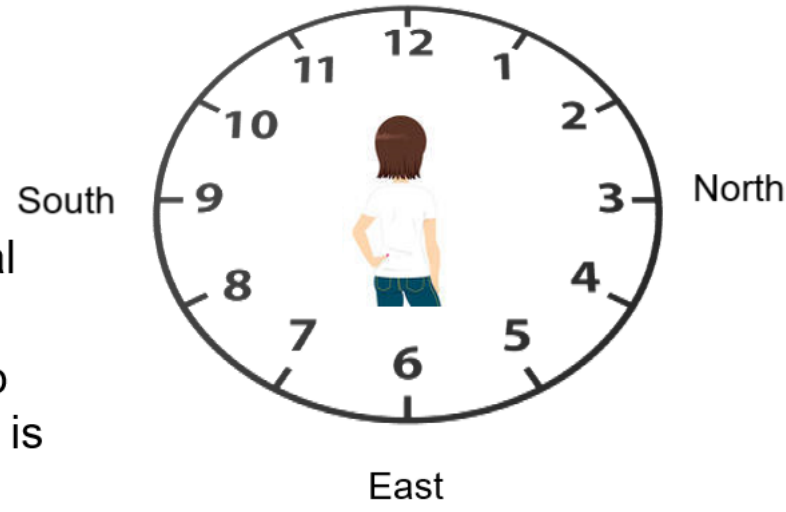

Figure 19. Example of training materials for the clock face directions.

Next, participants were provided with information regarding providing assistance to the robot through a rerouting task. Specifically, participants were informed that because the simulated robot in this study is still in development and testing phases, it may occasionally veer from its planned path through the simulated environment. Further, participants were told that, periodically, they would be asked if the robot had veered from its planned path. If the robot had veered from the path, participants would be responsible for rerouting the robot back to the planned path. Participants were also informed that rerouting the robot consisted of using a map provided to them, to draw a route back to the planned path via the shortest path while simultaneously avoiding sensitive areas of the 
environment. Sensitive areas of the environment were defined as buildings labeled as recreation centers and/or medical centers. Participants were provided with example routes that matched these criteria. Then participants were given an opportunity to practice rerouting (i.e., providing assistance to the robot), by drawing a route on a map located in the participant binder located on the desk of their workstation. In addition, participants were informed that the robot would not actually execute drawn rerouting paths. Instead, they were informed that the robot would store the information for executing a better path in future navigation scenarios. Once participants completed the rerouting practice task, they were directed back to their training presentation.

The next stage of the presentation contained information about answering SA regarding the robot questions, as well as responding to the NASA-TLX questionnaire. Once participants finished viewing this portion of the training presentation, they were asked to complete a training retention questionnaire to evaluate retention and comprehension of the training material.

Once participants completed the training retention questionnaire, they were asked to complete three practice missions in the MIX simulation. All three practice missions were approximately 3 minutes and 30 seconds in length and each practice mission included all elements of the experimental tasks (i.e., receiving robot-to-human information exchanges in the form of status updates, providing assistance to the robot via the rerouting task, answering SA regarding the robot questions). During the training 
missions, the change detection task included approximately 15 change events with an average event rate of one change every 5 seconds and a signal saliency of 2 icons changing per individual change event. The order of completion of the three practice missions was randomized within participants and counterbalanced across participants.

\section{Overhead map}

For each mission (training and experimental), participants were provided with an overhead map of the urban environment in which the simulated team was operating. This map listed each building located in the environment, and depicted the waypoints that comprised the robot's intended path through the environment, including the start and end points of the robot's route. In the development of each map for each study condition, care was taken to ensure that each of the routes that the robot traveled were roughly equivalent. In most instances, waypoints dictating where and how the simulated robot would move, were located in all four quadrants of the map. In addition, the design of the map involved ensuring that roughly the same number of buildings and the same number of building types were located in all four quadrants of the map. Finally, considerable effort was given to ensure that for each quadrant, the types of buildings located in direct proximity to one another (i.e., directly across the street from one another) were unique in almost all instances. Meaning that, in the upper left corner of the map there is only one instance in which a school is located directly across a street from a café, and so on. As such, if the robot were to give a waypoint status update which reported, "There is a 
school on one side and a café on the other", there is only one location in the upper left quadrant of the map that could match that description.

The map also provided a compass rose depicting the orientation of the map to the participants. For the 7UP, 13UP, 13UPLevel1 conditions, the compass rose depicted the four cardinal directions. For the 13UPlevel 2 condition, the compass rose depicted a clock face overlaid on the four cardinal directions. Finally, each map employed a grid system of numbers and letters. This system was intended to aid participants in answering SA regarding the robot questions. For example, the question, "Where is the robot?" could be answered as "F3". All maps were printed on 8 1/2 x 14, legal size paper and maps were provided to participants at the start of every mission. In addition, before beginning each mission, participants were given one minute to review each map. Figure 20 depicts an example of an overhead map provided in one of the experimental missions. Overhead maps for all of the missions are included in the appendices of this document. 


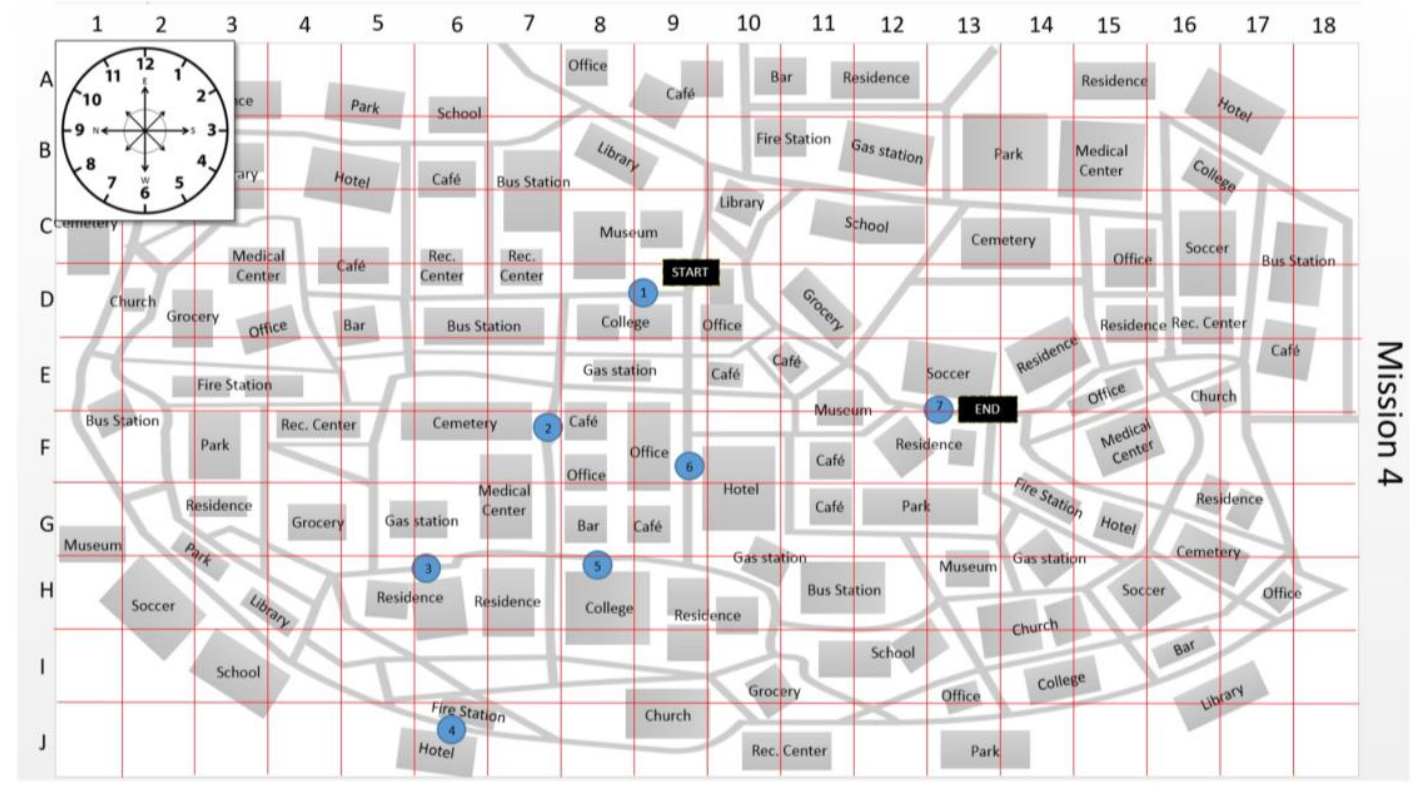

Figure 20. Overhead map used for the mission associated with the 13UPLevel2 condition. Blue circles represent the robot's planned waypoint route. The start and end points of the route are denoted. Note: Map was printed on $8 \frac{1}{2} \times 14$ legal size paper.

\section{$\underline{\text { Measures }}$}

\section{SA regarding the robot}

Throughout each mission (training and experimental), the participant was periodically probed with queries concerning level 1, level 2, and level 3 SA regarding the robot. Similar to the SAGAT method of SA measurement (Endsley, 1995b), the simulation briefly paused, the participants' screen temporarily blanked, and participants were asked to respond to SA questions that correspond to the three levels of SA regarding the robot. At each pause, three SA questions were presented from a bank of possible SA questions. See Table 5 for the bank of SA questions. Each question was intended to measure the participants' awareness regarding the robot, and environmental and task 
features. Each question represented an objective measure of SA with one correct answer. The presentation of SA questions was randomly timed throughout the study. Participant SA regarding the robot was probed once during each training mission, and twice during each experimental mission.

The scoring of the responses to the SA questions was as follows: correct responses to level $1 \mathrm{SA}$ questions $=1$ point, correct responses to level $2 \mathrm{SA}$ questions $=2$ points, and correct responses to level 3 SA questions $=3$ points. Points acquired for each SA question were then summed to provide an overall total score for the SA probe event. For missions in which SA was probed more than once (i.e., 7UP, 13UP, 13UPLevel1, and 13UPLevel2 conditions), scores for each SA probe event were summed to provide an overall SA regarding the robot score. In addition, SA regarding the robot scores were summed across conditions to provide an overall SA regarding the robot score for the experimental phase of the study. 
Table 5. Table of SA regarding the robot questions

\begin{tabular}{ll}
\hline SA regarding the robot & Question \\
\hline Level 1: Perception & Where is the robot? \\
& Between which two buildings is the robot currently \\
& located? \\
& Is the robot near any sensitive buildings? \\
& How much time is remaining on the countdown clock? \\
& Where is the robot? \\
Level 2: Comprehension & Thus far, has the robot veered from its planned waypoint \\
& path? \\
& Is the robot at the assigned waypoint? \\
& Has the robot's waypoint path, thus far, come into contact \\
& with any sensitive buildings? \\
& In what direction would the robot need to travel to get \\
& back to waypoint 1 (In cardinal directions)? \\
& In what direction would the robot need to travel to get to \\
& planned waypoint 3 (in Clock face directions)? \\
& At its current rate, is the robot going to reach the end of its \\
path in the time remaining? & If the robot proceeds to the next waypoint, will it \\
encounter any sensitive buildings? & \\
& If the robot travels East for 2 grid blocks from its current \\
location, will it encounter any sensitive buildings? & If the robot heads at 3 o'clock from its current location for \\
& 2 grid blocks, will it encounter any sensitive buildings? \\
\hline & Providing assistance to the robot
\end{tabular}

During the course of the robot's movement through the environment, the robot occasionally veered from its planned waypoint path by navigating to a different waypoint or series of waypoints. Performance on providing assistance to the robot was operationalized as the participants' ability to re-route the robot back to its planned path, by drawing a rerouting path on a physical map provided to the participants. When the simulation paused to ask SA regarding the robot questions, participants also were sked an 
additional question regarding rerouting the robot. Specifically, participants were asked the following question, "Based on its current location, does the robot need to be rerouted?" If participants answered yes, then they were directed to a map on which they could draw a path for the robot to navigate back to the planned waypoint path. Maps were located in the participant binders at the participant workstations. Figure 21 shows an example of a rerouting map provided to the participants during the 13UPLevel2 condition. The blue circle on this map denotes the planned waypoint to which participants were asked to reroute the robot, by drawing on the map. Figure 22 depicts a hypothetical rerouting path a participant could have drawn.

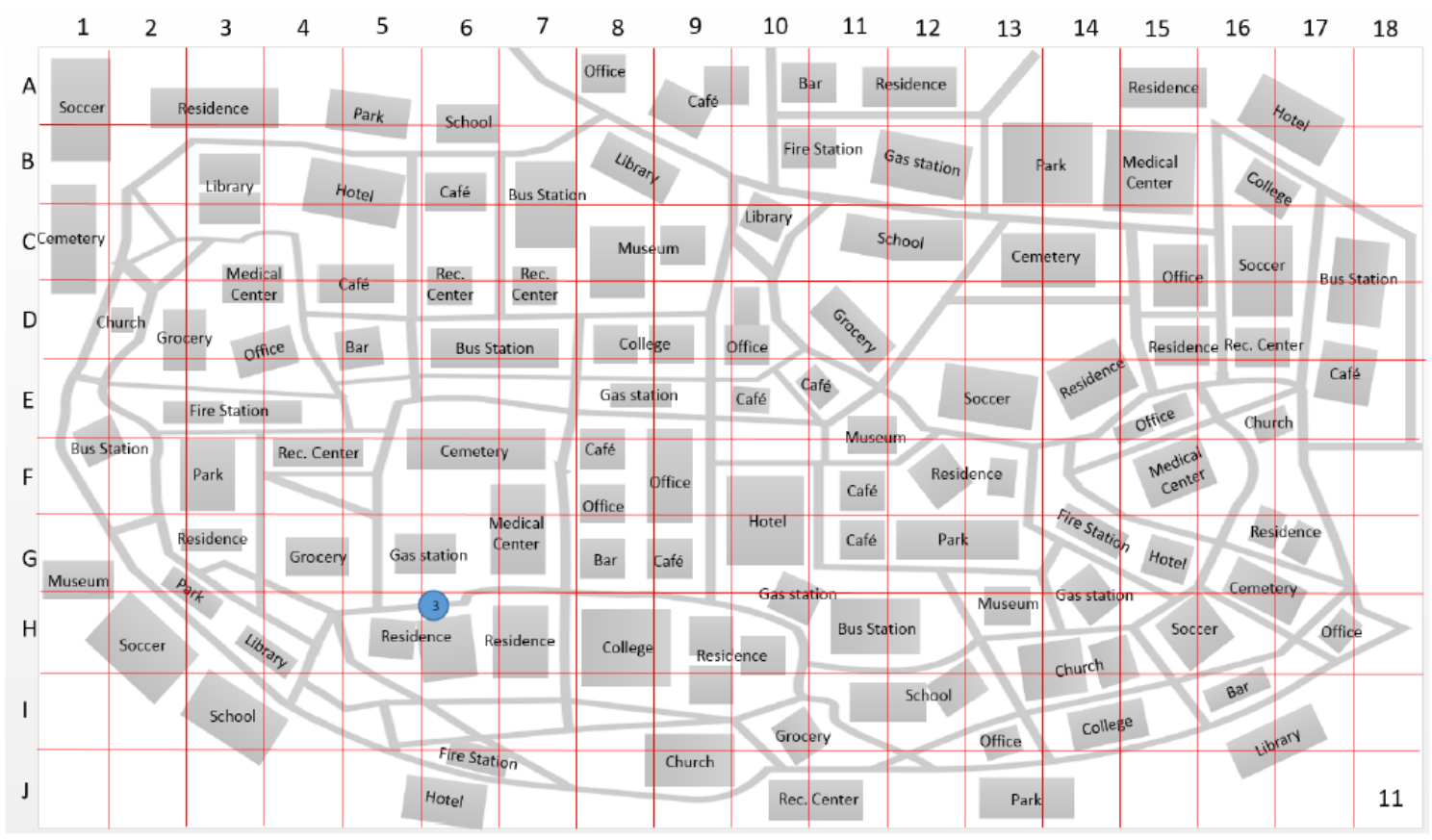

Figure 21. Rerouting map provided to participants during the 13UPLevel2 condition. The blue circle depicts planned waypoint number 3 in the robot's planned waypoint path. 


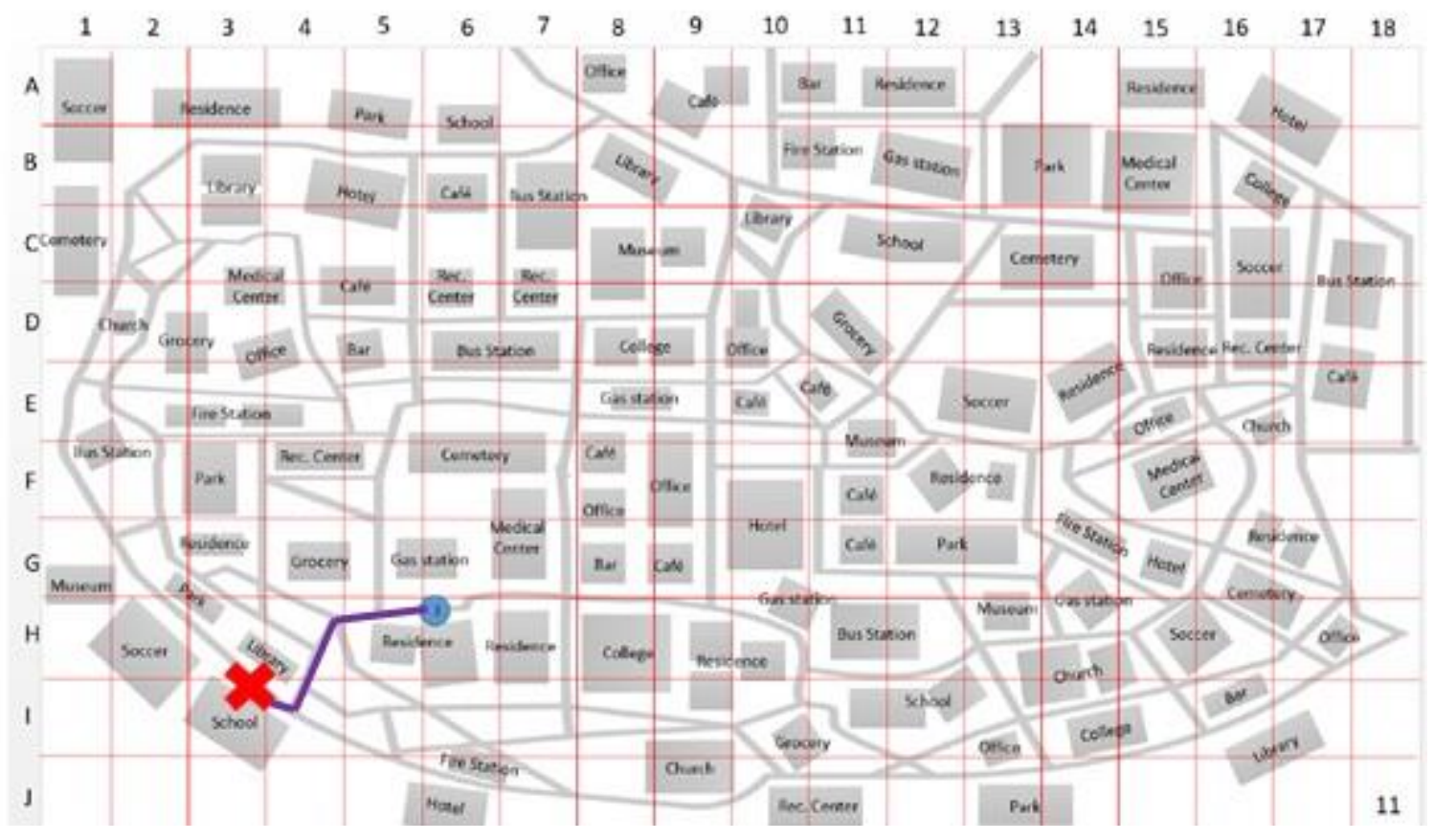

Figure 22. Example of a rerouting drawing completed in 13UPLevel2 condition. The blue circle depicts planned waypoint number 3 in the robot's planned waypoint path. The red $\mathrm{X}$ denotes the robot's location at the time of the probe event. The route in purple denotes a rerouting path that participants could have drawn.

Participants were instructed that rerouting the robot involved drawing the shortest path back to the planned path, while simultaneously avoiding sensitive buildings in the environment. Sensitive buildings included recreation centers and medical centers.

Participants were instructed to avoid drawing a path that utilized roads that crossed in direct proximity to a sensitive building. Participant performance in rerouting the robot back to its planned path was measured according to the instructions outlined in table 6 . 
Table 6. Table listing instructions for scoring each rerouting the robot map.

Scoring the rerouting maps is as follows:

1. If the route begins in the correct starting location 0 points

1a. If the route does not begin in the correct starting location 20 points

2. Count the number of grid blocks the drawn route crosses \# of blocks =\# of through.

points

3 Does the route utilize roads that cross in direct proximity to 3 points for each any sensitive buildings?

4 Did the participant not draw a route when they should have 20 points done so?

5 Did the participant draw a route when they should not have 20 points done so?

6 Sum these points together to obtain a rerouting score for each rerouting map.

Each rerouting map was scored according to the instructions listed above ${ }^{5}$. Because the above scale is additive in which points are awarded for poor performance, (e.g., routing near sensitive buildings results in 3 points awarded), the scale is reverse scored.

Therefore, lower scores derived using this scale are indicative of better assistance provided to the robot than higher scores.

Similar to the SA regarding the robot measure, providing assistance to the robot via rerouting the robot, was assessed once during each training mission (for a total of 3 rerouting maps), and twice during each experimental mission (for a total of 8 rerouting maps). In addition, a rerouting map was created for the practice rerouting task completed

${ }^{5}$ According to the scoring instructions in table 6, the hypothetical path drawn in Figure 24 would have been scored as a 4 on the measure of providing assistance to the robot. 
in the training phase of the study. As such, a total of 12 maps were scored for each participant. For each study condition, scores for both maps were summed to provide an overall assessment of performance in providing assistance to the robot for the condition. In addition, scores on each study condition were summed to provide an overall assessment of performance in providing assistance to the robot for the experimental phase of the study. All rerouting maps are provided in the appendices of this document.

\section{Corsi block tapping task}

Participants completed a computerized measure of visuospatial working memory via the Corsi block tapping task (Corsi, 1972). The Corsi block tapping task involved watching a sequence of blocks presented on the computer screen light up with color in a random order. Participants were then asked to mimic the order in which the blocks lit up by clicking on each block with their mouse cursor in sequential order. As participants progressed through the task, the sequence of blocks became progressively longer and consequently more difficult to reproduce. Participants continued this procedure until performance began to degrade. Visuospatial working memory performance was measured as the number of blocks in the sequence that participants could reproduce. Figure 23 shows an example of the task stimuli. 


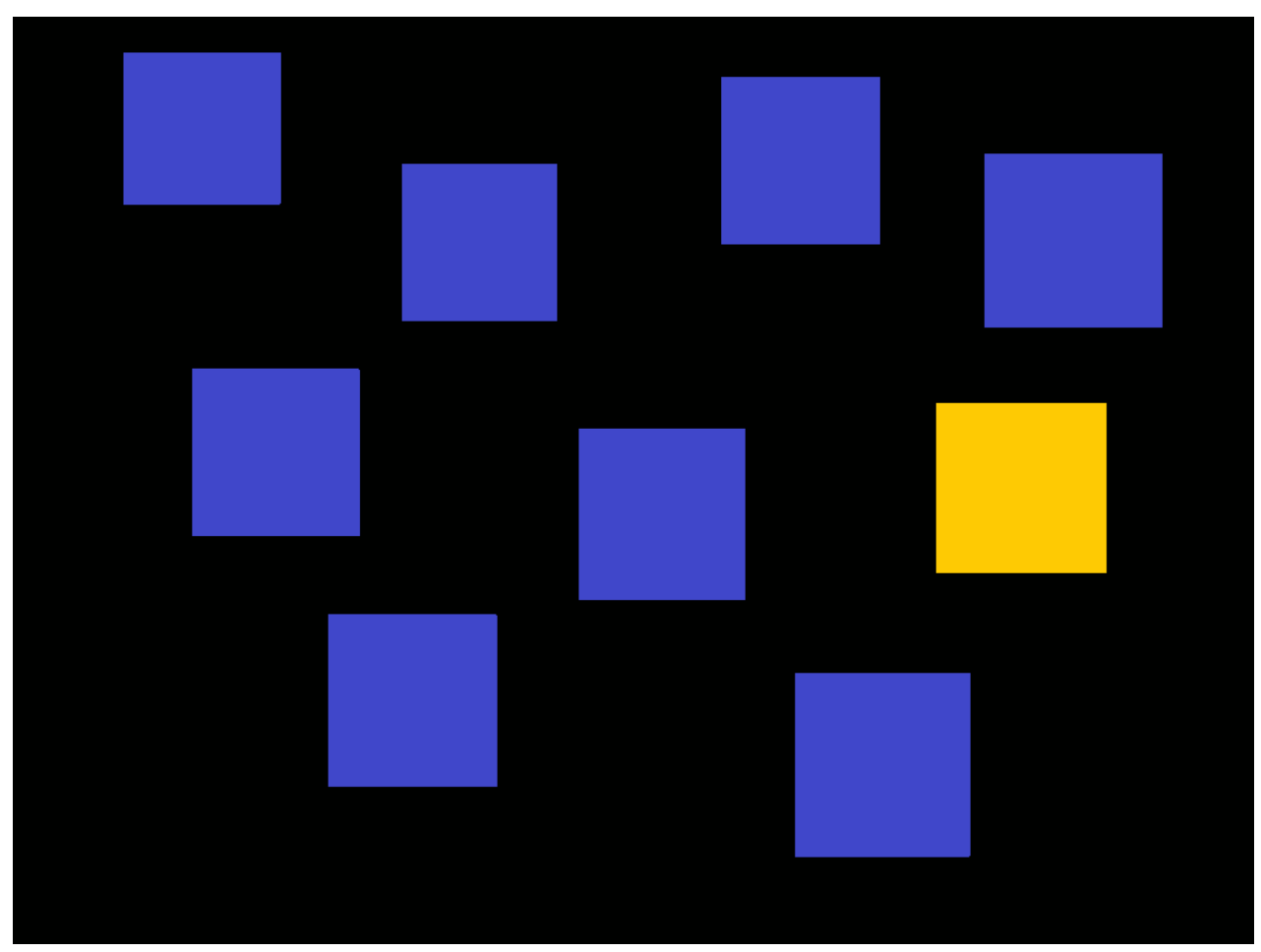

Figure 23. Example of Corsi block tapping task stimuli.

\section{Perspective taking /Spatial Orientation Test}

Participants also completed the Perspective taking/Spatial orientation test (Hegarty \& Waller, 2004; Kozhevnikov \& Hegarty, 2001). This test is a measure of individual ability to imagine different perspectives or orientations in space. For each item, participants were presented with a picture depicting an array of objects and an "arrow circle" with a question about the direction between some of the objects (see Figure 24). 

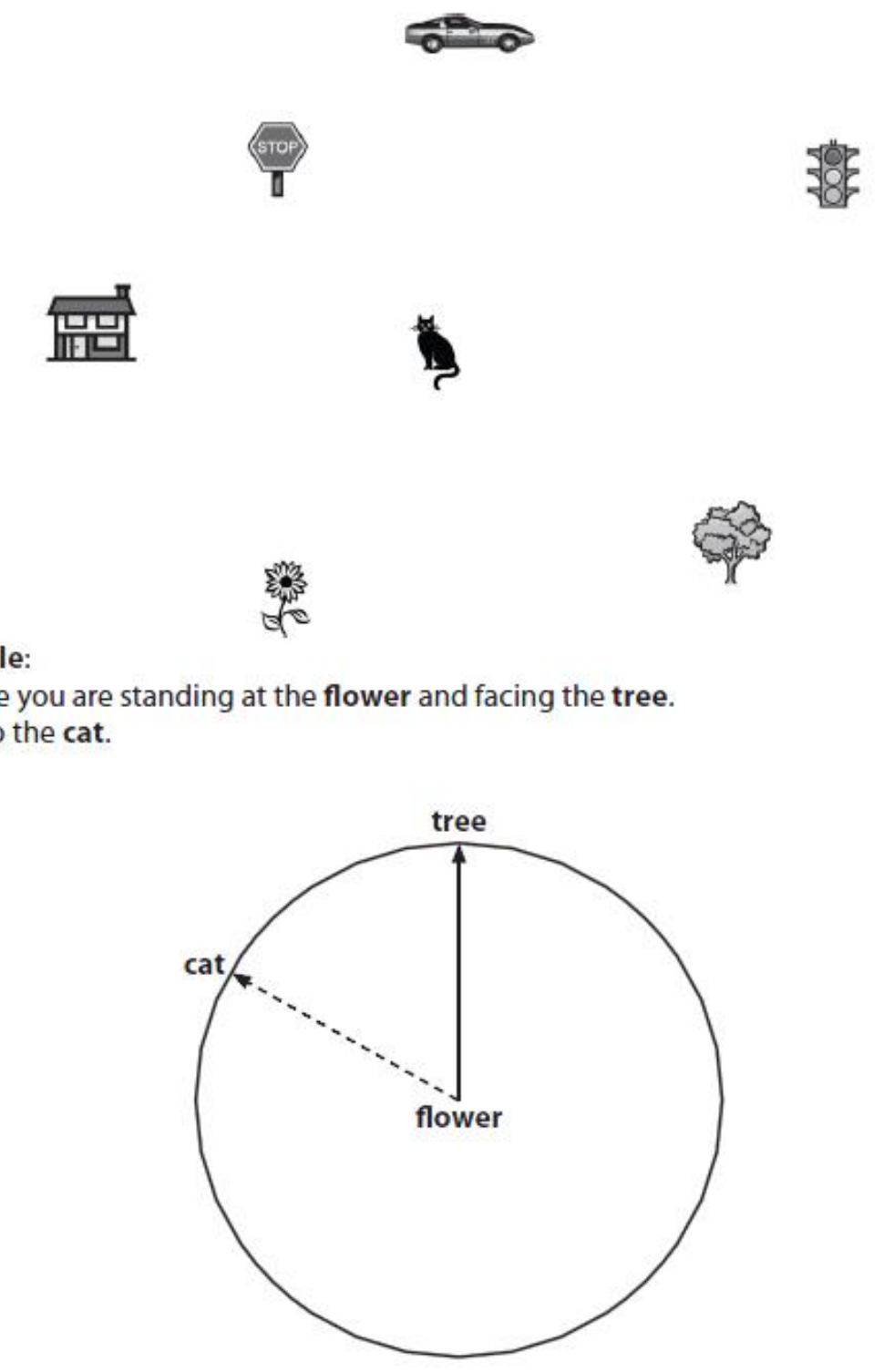

Figure 24. Example item from the Perspective taking/Spatial orientation test. 
Participants were asked to imagine that they were standing at one object in the array (which denoted the center of the circle), and facing another object depicted at the top of the circle. Participants were then asked to draw an arrow from the center of the circle in the direction of a third object from the perspective described prior. Participants were given 5 minutes to complete 12 items in this test. Each item in this measure was scored by the absolute deviation in degrees between the participants' response and the correct direction to the target (absolute directional error). As such, this measure is reverse scaled as more directional error is indicative of worse performance than less directional error. A participant's total score on this measure was given by the average deviation across all attempted items.

\section{NASA-TLX}

During the course of each mission, participants were asked to complete a computerized version of the NASA-TLX, a subjective measure of global workload with 6 subscales: mental demand, physical demand, temporal demand, effort, frustration, and performance (Hart \& Staveland, 1988). At approximately the mid-point of each mission, the task was paused and participants were asked to provide a rating of their perceived mental workload along the dimensions described above via a scale ranging from 0 (Low) to 100 (high) for the first five dimensions, and from 0 (poor) to 100 (good) for the performance dimension. For this study, the weighting measure of paired comparisons among the subscales was not included. 


\section{Biographical data form}

Participants completed a biographical data form which contained questions pertaining to biographical information like, age, gender, prior military experience, as well as known color vision deficiencies. In addition, participants were asked a series of questions pertaining to their prior familiarity and experience with the robotics domain as well as robots intended for specific types of uses (e.g., robot s for the home, robots for therapy/medical purposes, military robots). Participants rated their level of familiarity and/or experience on 6 point Likert-type scales which ranged from 1 (No experience, Not familiar) to 6 (Very experienced, Very familiar).

\section{Training retention questionnaire}

Finally, participants completed a questionnaire intended to assess their retention and understanding of the information contained in the training materials. Participants responded to 10 multiple choice items that pertained to each of the major topic areas covered in the training materials, including reading the compass rose, determining clock face directions, re-routing the robot, completing the change detection task, and the robotto-human information exchanges. Each question presented 4 answer choices in which only one was the correct answer. Participants' scores on the training retention measure were determined as the number of items answered correctly. 


\section{Change detection task performance}

While receiving status updates from the robot, participants were tasked with completing a dual task in the form of a change detection task. Participant performance on the change detection task was measured as the number of change events correctly detected (i.e., the number of events detected and correctly classified as appear, disappear, or movement events) out of the total number of events present in each study condition.

\section{Procedure}

Once in the lab, participants were presented with the informed consent form that included the details of the study, information on the rights of research participants, the proposed study timeline, and contact information for the research team, faculty advisor, and institutional review boards (both UCF and Army Research Lab). After reviewing the form, participants were given the opportunity to ask for clarification on any of the study details and/or ask more questions about the research. Once this opportunity passed, and all questions and/or concerns were addressed, participants were asked if they would like to continue their participation in the study. Participants indicated their consent to begin participation in the study through a verbal confirmation and by signing their name on the informed consent form.

Once the informed consent form was signed, participants moved into the biographical data phase of the study. During this phase, participants completed the 
biographical data form, the Perspective taking/Spatial orientation test, and the Corsi block tapping task. Once these measures were completed, participants moved into the training phase of the study. The training phase of the study included viewing a narrated PowerPoint presentation with breaks included to provide opportunities to practice the change detection task, and the rerouting task, as well as answering questions regarding the compass rose. Once participants finished viewing the narrated PowerPoint presentation, they were asked to complete 3 training missions in the MIX testbed. Then, participants took a five minute break before beginning the next phase of the study.

After the 5 minute break had elapsed, participants moved into the experimental phase of the study. During this phase, participants completed four experimental mission scenarios. During two of the scenarios, participants received robot-to-human information exchanges in the form of waypoint status updates (i.e., the 7UP and 13UP conditions). In the third mission scenario, participants received robot-to-human information exchanges in the form of waypoint status updates and traveling status updates with Level 1 VPT (i.e., the 13UPLevel1 condition). In the fourth mission scenario, participants received robot-to-human information exchanges in the form of waypoint status updates and traveling status updates with Level 2 VPT context (i.e., the 13UPLevel2 condition). The order in which participants completed each scenario was pseudo-randomized, utilizing two Latin square designs (see discussion of study design). Each participant was assigned to complete one of 8 possible orders to complete each mission (e.g., 4, 1, 2, 3; 1, 4, 3, 2; 
etc.). While completing each scenario, participants were asked to perform the change detection task, answer SA regarding the robot questions, fill out the NASA-TLX measure, and provide assistance to the robot via the rerouting task when necessary. Once the participants completed all four of the experimental mission scenarios, participants moved into the debriefing phase of the study.

During the debriefing phase of the study, participants were provided with a post participation information form. This form included details concerning the goals of the study as well as contact information for the researcher and institutional review boards in the event that they needed to report a problem, find out more, or wished to learn the results of the study at a later date. Also, participants were provide with an optional researcher evaluation form, which provided a means to evaluate the performance of the researcher and the research assistants associated with this study. If participants chose to complete this form, they were asked to submit the form to the University of Central Florida's Psychology Department. Participants were also asked not to share the details of the study with their friends or classmates should those individuals wish to participate in the study as well. Once participants were provided with this information, they were thanked for their time and granted course credit for their participation via Sona Systems. Figure 25 provides a flow chart depicting the procedure of the study. The time to complete the study was approximately 2 hours and 30 minutes and participants received 2.5 Sona credits for completing the study. 


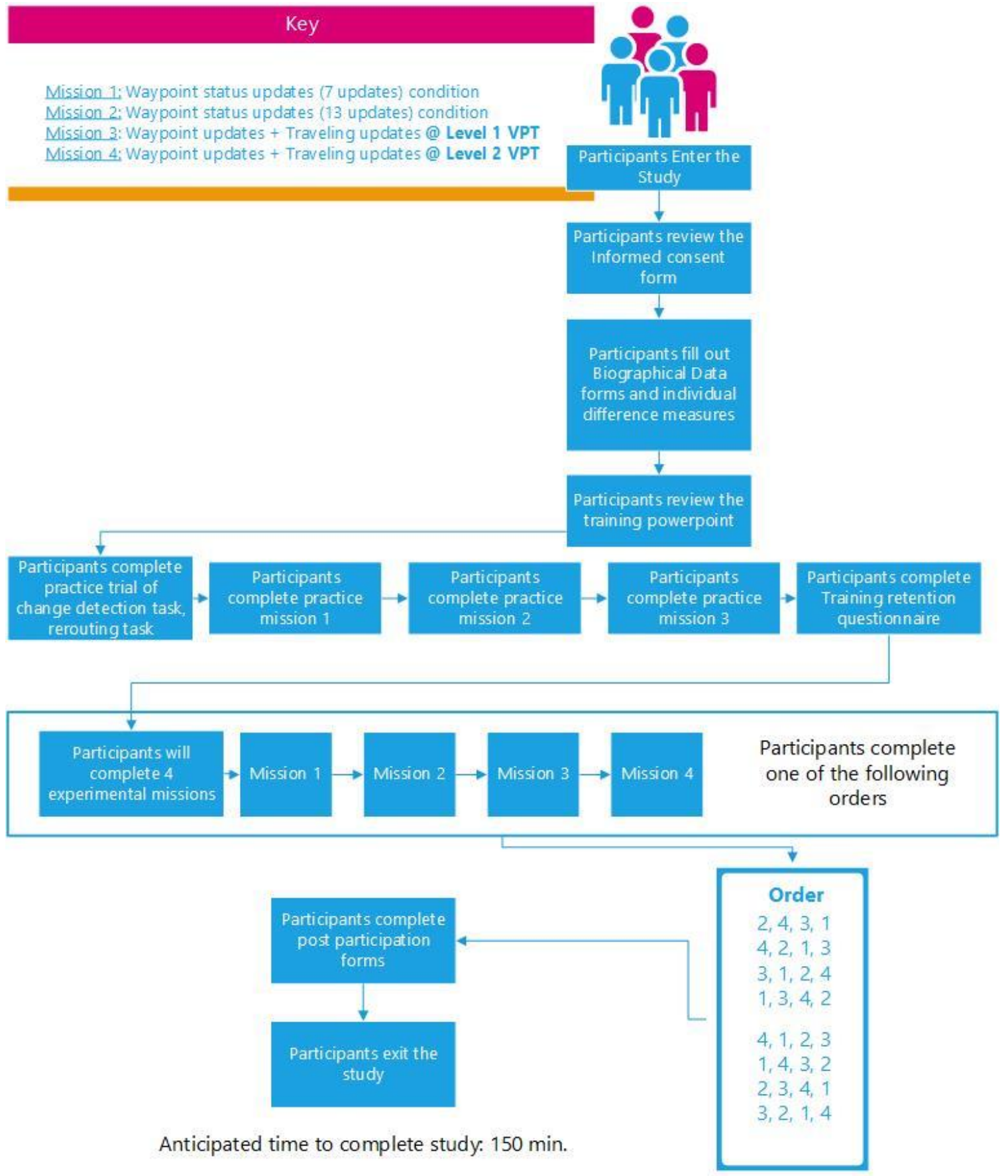

Figure 25. Flow chart of study procedure. 


\title{
CHAPTER FOUR: RESULTS
}

\author{
$\underline{\text { Data screening and reduction }}$
}

Eighty-four participants (62 males and 22 females) participated in this study. Of the 84 participants, 8 were excluded from the analyses in the first screening of the data. Data for one participant was accidentally lost, one participant decided not to complete the study and was generally behaving in a disruptive manner, and two participants fell asleep (one while completing the experimental tasks and one while viewing the training PowerPoint presentation). The remaining four participants were excluded because they were not presented with all of the within-subjects study conditions due to mistakes in the administration of the study. Upon a second screening of the data, it was decided to control for the effects of participants' biological sex in the analyses. Appendix I of this document contains more detail about the influence of participant sex in the analyses and the removal of the female participants.

The final sample included 56 males with ages ranging from 18 to 29 ( $M=18.89$, $S D=3.412$ ). Age for three participants was not recorded due to experimenter mistakes in the administration of the demographic questionnaires of the study. Of the 56 participants, five reported that English was not their native language. Only one participant reported a known color vision problem. One participant reported prior active duty military experience, one participant reported that they had completed five weeks of basic training, and two participants indicated that they had four years of JROTC experience. Participants 
were asked to report their knowledge and familiarity regarding robots or the robotics domain on a 6 point Likert-type scale. All participants reported low familiarity with robots $(M=2.44, S D=1.162)$. Similarly, participants were asked to rate their experience (i.e., having worked with or come into contact with) robots, on a 6 point Likert-type scale. All participants similarly reported low experience with robots $(M=2.13$, $S D=1.268)$.

\section{$\underline{\text { Demographic variables }}$}

All analyses were conducted using IBMs SPSS software version 23. Demographic variables for the sample are presented in Table 7. Pearson's product-moment correlation was used to assess whether significant correlations were present among the demographic variables: GPA, self-reported familiarity with robots, and self-reported experience with robots. A significant positive Pearson's correlation was found between participant age and self-reported familiarity with robots and/or the robotics domain, suggesting that older participants reported more familiarity with robots than younger participants. Also, a significant positive Pearson's correlation was found between participants' self-reported familiarity with robots (i.e., knowledge or familiarity with robots or the robotics domain) and self-reported experience with robots (i.e., having worked with or come into contact with robots). Participants who reported high familiarity with robots were also more likely to report greater experience working with or coming into contact with robots, than students who reported low familiarity with robots. 
Table 7. Descriptive statistics and inter-correlations among demographic variables.

\begin{tabular}{lcccccc}
\hline Variable & $\boldsymbol{M}$ & $\boldsymbol{S D}$ & $\mathbf{1 .}$ & $\mathbf{2 .}$ & $\mathbf{3 .}$ & $\mathbf{4 .}$ \\
\hline 1. Age (years) & 18.890 & 3.412 & 1 & & & \\
$\begin{array}{l}\text { 2. GPA (0.00-4.00) } \\
\begin{array}{l}\text { 3. Experience with robots } \\
\text { (1-6) }\end{array}\end{array}$ & 3.450 & 0.820 & -.061 & 1 & & \\
$\begin{array}{l}\text { 4. Familiarity with robots } \\
(1-6)\end{array}$ & 2.130 & 1.268 & .222 & .104 & 1 & \\
& 2.440 & 1.162 & $.304^{*}$ & .103 & $.757^{* *}$ & 1 \\
\hline
\end{tabular}

Note: Self-reported experience with robots (i.e., having worked with/come into contact with robots) and self-reported familiarity with robots were rated on Likert-type scales where, $1=$ No experience or Not familiar to $6=$ Very experienced or Very familiar. ${ }^{*} p<.05,{ }^{* *} p<.01$.

Preliminary analyses: Demographic variables and assignment to order conditions

A Chi Square test revealed that there was not a significant relationship between assignment to order conditions and participant age, $X^{2}(1, \mathrm{n}=53)=68.661, p=.292$, Crammer's $V=0.430$. In addition, a series of between subjects one-way ANOVAs were conducted to determine if participants differed significantly from one another on GPA, familiarity with robots, and experience with robots, across order conditions. Significant differences across order conditions were not found in participant: GPA $F(7,42)=1.643$, $p=.150$; familiarity with robots $F(7,44)=1.017, p=.432$; or experience with robots $F(7$, $44)=0.336, p=.933$. 
Preliminary analyses: Dependent variables and assignment to order conditions

A one-way between-subjects ANOVA with the order in which participants completed the study conditions as the between-subjects variable and SA regarding scores as the dependent variable, was conducted to test for significant differences in scores on the measure of SA regarding the robot due to the order in which participants completed each within-subjects study condition. The ANOVA test revealed that there were no significant differences in SA scores due to the order of completion of the study conditions, $F(7,48)=1.042, p=.415$.

A one-way between-subjects ANOVA, with the order in which participants completed the study conditions as the between-subjects variable and scores on the measure of providing assistance to the robot as the dependent variable, was conducted to test for significant difference in scores on the measures of assistance provided to the robot due to the order in which participants completed each study condition. Significant differences in assistance scores due to the order of completion of the within-subjects study conditions were not found $F(7,48)=0.742, p=.637$.

Preliminary analyses: Mental workload and assignment to order conditions

A between-subjects MANOVA, with the order in which participants completed the study conditions as the between-subjects variable, and scores on the NASA-TLX measures as the dependent variables, was conducted to test for significant differences in scores on the measure of perceived mental workload due to the order in which 
participants completed each of the study conditions. Significant differences in scores on the measure of perceived mental workload (NASA-TLX) due to the order in which participants completed each within-subjects study condition were not found Wilks Lambda $=0.551, F(4,45)=1.052, p=.403$, Pillai's Trace $=.525, F(4,45)=1.035, p=$ .424 .

$\underline{\text { Preliminary analyses: Mental workload across within-subjects conditions }}$

A one-way repeated measures ANOVA was conducted to test for significant differences in scores on the measures of participants' perceived mental workload (i.e., NASA-TLX) across the within-subjects study conditions. Significant differences in scores were not found, Wilks Lambda $=0.980, F(3,53)=0.363, p=.780$, indicating that perceived mental workload induced by the experimental tasks were roughly the same across study conditions (see Table 8). Participants did not perceive any one experimental mission to induce more mental workload than any other. In addition, mean scores on the measure of perceive mental workload, across study conditions were similar to those established as a indicating a "Medium level" of perceived mental workload by Cinaz, Bert, La Marca, and Tröster (2013) in controlled laboratory tasks.

Table 8. Table of means and standard deviations for scores on the NASA-TLX measure.

\begin{tabular}{llcc}
\hline Mission & Condition & $\begin{array}{c}\text { NASA-TLX } \\
\text { Mean }\end{array}$ & Std. Deviation \\
\hline Mission 1 & 7UP & 60.61 & 11.373 \\
Mission 2 & 13UP & 59.57 & 10.747 \\
Mission 3 & 13UPLevel1 & 60.31 & 10.947 \\
Mission 4 & 13UPLevel2 & 60.57 & 9.479 \\
\hline
\end{tabular}


Hypothesis $\mathrm{H} 1$

H1: Higher levels of SA regarding the robot will be positively associated with superior human assistance provided to the robot.

Hierarchical multiple regression was used to test Hypothesis H1. Specifically, hierarchical multiple regression was used to assess the relationship between scores on the measure of SA regarding the robot and scores on the measure of assistance provided to the robot, while controlling for individual difference variables. For this test, aggregate scores of SA regarding the robot and assistance provided to the robot across all four experimental missions were used. In addition, scores on the measure of providing assistance to the robot were reversed before conducting the analyses to aid in the interpretation of the results. Scores on the measures of visuospatial working memory (Corsi block tapping task), spatial orientation (Perspective taking/Spatial orientation test), and prior robotics experience, were entered at Step 1 and explained 10\% of the variance in scores on the measure of providing assistance to the robot, $R^{2}=.100, F(4,51)=1.422$, $p=.240$. After scores on the measure of SA regarding the robot were entered at Step 2, the overall model explained $33.9 \%$ of the variance in scores on the measure of assistance provided to the robot, $R^{2}=.339, F(5,50)=5.132, p=.001$, representing a significant change in the amount of variance explained by the model, $R^{2}$ change $=.239, p<.001$. In addition, after controlling for the scores on the measures of individual differences, the only variable to make a significant unique contribution to explaining variance in scores 
on the measure of providing assistance to the robot, was scores on the measure of SA regarding the robot, beta $=1.738, p<.001$. As such, Hypothesis H1, which stated that higher levels of SA regarding the robot will be positively associated with superior human assistance provided to the robot, was supported.

\section{Hypotheses $\mathrm{H} 2$ through $\mathrm{H} 5$}

\section{Summary of analyses}

To analyze Hypotheses $\mathrm{H} 2$ through $\mathrm{H} 5$, several planned contrasts of means scores on the dependent measures between within-subjects study conditions were made. Specifically, a comparison of scores were made between the 7UP condition and the 13UP condition, on the measures of SA and providing assistance to the robot. A comparison of scores on the same dependent measures were made between the 13UPLevel1 condition and the 13UPLevel 2 condition. Two additional contrasts between mean scores on the measures of SA regarding the robot and providing assistance to the robot also were conducted between the 13UP and the 13UPLevel1 conditions. The additional planned contrasts were intended to examine the benefit of adding VPT context to robot-to-human information exchanges, as opposed to simply adding more robot-to-human information exchanges. Table 9 presents a summary of the comparisons conducted to test Hypotheses $\mathrm{H} 2$ through $\mathrm{H} 5$. Appendix $\mathrm{H}$ also provides a detailed summary of the planned analyses for Hypotheses H2 through H5. 
Table 9. Summary of comparisons conducted to analyze Hypotheses H2 through H5.

\begin{tabular}{|c|c|c|c|c|}
\hline Hypothesis & & $\begin{array}{l}\text { Dependent } \\
\text { measure }\end{array}$ & & Conditions \\
\hline H2 & $\begin{array}{c}\text { Comparison } \\
\text { of }\end{array}$ & $\begin{array}{l}\text { SA regarding the } \\
\text { robot scores }\end{array}$ & Between & $\begin{array}{l}\text { 7UP condition } \\
\& \\
\text { 13UP condition }\end{array}$ \\
\hline H3 & $\begin{array}{c}\text { Comparison } \\
\text { of }\end{array}$ & $\begin{array}{l}\text { Providing } \\
\text { assistance to the } \\
\text { robot scores }\end{array}$ & Between & $\begin{array}{l}\text { 7UP condition } \\
\& \& \\
\text { 13UP condition }\end{array}$ \\
\hline H4 & $\begin{array}{c}\text { Comparison } \\
\text { of }\end{array}$ & $\begin{array}{l}\text { SA regarding the } \\
\text { robot scores }\end{array}$ & Between & $\begin{array}{l}\text { 13UPLevel1 condition } \\
\& \& \\
\text { 13UPLevel2 condition }\end{array}$ \\
\hline H5 & $\begin{array}{c}\text { Comparison } \\
\text { of }\end{array}$ & $\begin{array}{l}\text { Providing } \\
\text { assistance to the } \\
\text { robot scores }\end{array}$ & Between & $\begin{array}{l}\text { 13UPLevel1 condition } \\
\& \& \\
\text { 13UPLevel2 condition }\end{array}$ \\
\hline $\begin{array}{l}\text { Additional } \\
\text { contrast } 1\end{array}$ & $\begin{array}{c}\text { Comparison } \\
\text { of }\end{array}$ & $\begin{array}{l}\text { SA regarding the } \\
\text { robot scores }\end{array}$ & Between & $\begin{array}{l}\text { 13UP condition } \\
\quad \& \\
\text { 13UPLevel1 condition }\end{array}$ \\
\hline $\begin{array}{l}\text { Additional } \\
\text { contrast } 2\end{array}$ & $\begin{array}{c}\text { Comparison } \\
\text { of }\end{array}$ & $\begin{array}{l}\text { Providing } \\
\text { assistance to the } \\
\text { robot scores }\end{array}$ & Between & $\begin{array}{l}\text { 13UP condition } \\
\& \& \\
\text { 13UPLevel1 condition }\end{array}$ \\
\hline
\end{tabular}

$\underline{\text { Hypotheses two and four: SA related hypotheses }}$

H2: Robot-to-human information exchanges will be positively associated with situation awareness regarding the robot, such that more exchanges should be associated with better SA regarding the robot than fewer exchanges. 
H4: Robot-to-human information exchanges that include higher levels of VPT context will lead to higher levels of SA regarding the robot than robot-to-human information exchanges that include lower levels of VPT context.

A one-way repeated measures ANOVA with a sample size of 56, was conducted to test for significant differences in mean scores on the measure of SA across withinsubjects study conditions. The test revealed a significant main effect for condition, Wilks Lambda $=0.636, F(3,53)=10.131, p<.001$, partial eta squared $=.364$, suggesting that, significant differences between mean scores on the measures of SA across withinsubjects study conditions were present. Planned contrasts of mean SA scores were conducted to test Hypotheses $\mathrm{H} 2$ and $\mathrm{H} 4$.

\section{Hypothesis H2 contrast}

The contrast between mean scores in the 7UP condition $(M=8.821)$ and 13UP condition $(M=7.875)$, was not significant, $p=.064$. Further, mean scores trended in the opposite of the hypothesized direction. Hypothesis $\mathrm{H} 2$, which stated that more robot-tohuman information exchanges would be associated with better SA regarding the robot than fewer robot-to-human information exchanges, was not supported.

\section{Hypothesis H4 contrast}

A contrast was also conducted to examine differences in mean scores on the measure of SA between the 13UPLevel1 condition and the 13UPLevel2 condition. A 
statistically significant difference was found between mean scores on the measure of SA between the 13UPLevel1 condition $(M=7.643)$ and the 13 UPLevel 2 condition $(M=$ $6.179), p=.002$. However, mean scores trended in the opposite of the hypothesized direction. As such, Hypothesis H4, which stated that robot-to-human information exchanges that include Level 2 VPT context will be associated with higher levels of SA regarding the robot, than robot-to-human information exchanges that include Level 1 VPT context, was not supported.

\section{Additional contrast 1}

Finally, a contrast was conducted to examine differences in mean scores on the measure of SA between the 13UP and the 13UPLevel 1 conditions. The difference between mean scores in the 13UP condition $(M=7.875)$ and the 13UPLevel 1 condition $(M=7.643)$ was not statistically significant, $p=.637$. Mean scores similarly trended in the opposite of the predicted direction. Table 10 and Figure 26 present mean scores on the measure of SA across experimental missions.

Table 10. Table of means and standard errors for SA regarding the robot scores across study conditions.

\begin{tabular}{llccc}
\hline Mission & Condition & $\begin{array}{c}\text { SA } \\
\text { Mean }\end{array}$ & Std. Error & N \\
\hline Mission 1 & 7UP & 8.821 & 0.363 & 56 \\
Mission 2 & 13UP & 7.875 & 0.365 & 56 \\
Mission 3 & 13UPLevel 1 & 7.643 & 0.384 & 56 \\
Mission 4 & 13UPLevel 2 & 6.179 & 0.363 & 56 \\
\hline
\end{tabular}




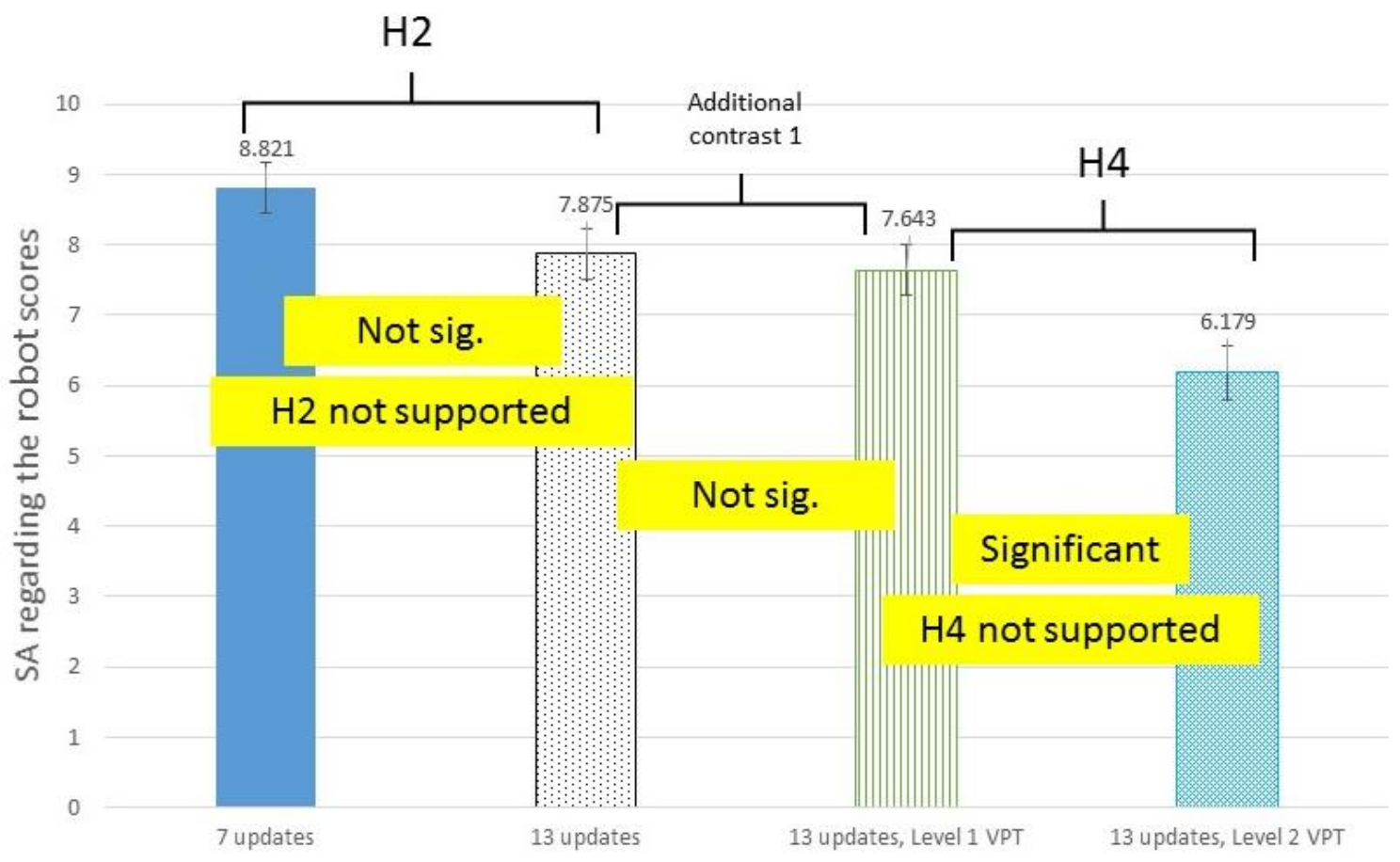

Figure 26. Pattern of mean scores on the measure of SA across within-subjects conditions and planned contrasts.

Hypotheses three and five: Providing assistance to the robot hypotheses

H3: Robot-to-human information exchanges will be positively associated with human assistance provided to the robot, such that more exchanges should be associated with better human assistance provided to the robot than fewer exchanges.

H5: Robot-to-human information exchanges that include higher levels of VPT context will lead to better human assistance provided to the robot, than robot-to-human information exchanges that include lower levels of VPT context. 
A one-way repeated measures ANOVA was conducted to test for significant differences in mean scores on the measure of providing assistance to the robot across within subjects study conditions. The test revealed a significant main effect for condition, Wilks Lambda $=0.179, F(3,53)=80.987, p<.001$, partial eta squared $=.821$, suggesting that significant differences between mean scores on the measure of providing assistance to the robot across within-subjects study conditions were present. Planned contrasts of mean providing assistance to the robot scores were conducted to test Hypotheses $\mathrm{H} 3$ and H5.

\section{Hypothesis H3 contrast}

A contrast between mean scores on the 7UP condition $(M=15.571)$ and the 13UP condition $(M=9.393)$ was significant, $p<.001$. However, mean scores trended in the opposite of the hypothesized direction. Hypothesis H3, which stated that more robot-tohuman information exchanges would be associated with better assistance provided to the robot than fewer robot-to-human information exchanges, was not supported.

\section{Hypothesis H5 contrast}

A contrast also was conducted to examine differences in mean scores on the measure of providing assistance to the robot between the 13UPLevel 1 condition and the 13UPLevel2 condition. A statistically significant difference was found between mean scores on the measure of providing assistance to the robot between the 13UPLevel 1 condition $(M=27.964)$ and the 13UPLevel 2 condition $(M=18.375), p<.001$. However, 
mean scores also trended in the opposite of the hypothesized direction. As such, Hypothesis H5, which stated that robot-to-human information exchanges that included Level 2 VPT context would be associated with higher quality of assistance provided to the robot than robot-to-human information exchanges that included Level 1 VPT context, was not supported.

\section{Additional contrast 2}

Finally, a comparison was conducted to examine differences in mean scores on the measure of providing assistance to the robot between the 13UP condition and the 13UPLevel 1 condition. The difference between mean scores in the 13UP condition $(M=$ 9.393) and the 13UPLevel 1 condition $(M=27.964)$ was statistically significant, $p<.001$, but trended in the opposite of the predicted direction Table 11 and Figure 27 present mean scores on the measure of assistance provided to the robot for each study condition.

Table 11. Table of means and standard errors for scores on the measure of providing assistance to the robot across study conditions.

\begin{tabular}{llccc}
\hline Mission & Condition & $\begin{array}{c}\text { Providing } \\
\text { assistance } \\
\text { Mean }\end{array}$ & Std. Error & N \\
\hline Mission 1 & 7UP & 15.571 & 0.902 & 56 \\
Mission 2 & 13UP & 9.393 & 0.960 & 56 \\
Mission 3 & 13UPLevel1 & 27.964 & 0.970 & 56 \\
Mission 4 & 13UPLevel2 & 18.375 & 1.578 & 56 \\
\hline
\end{tabular}




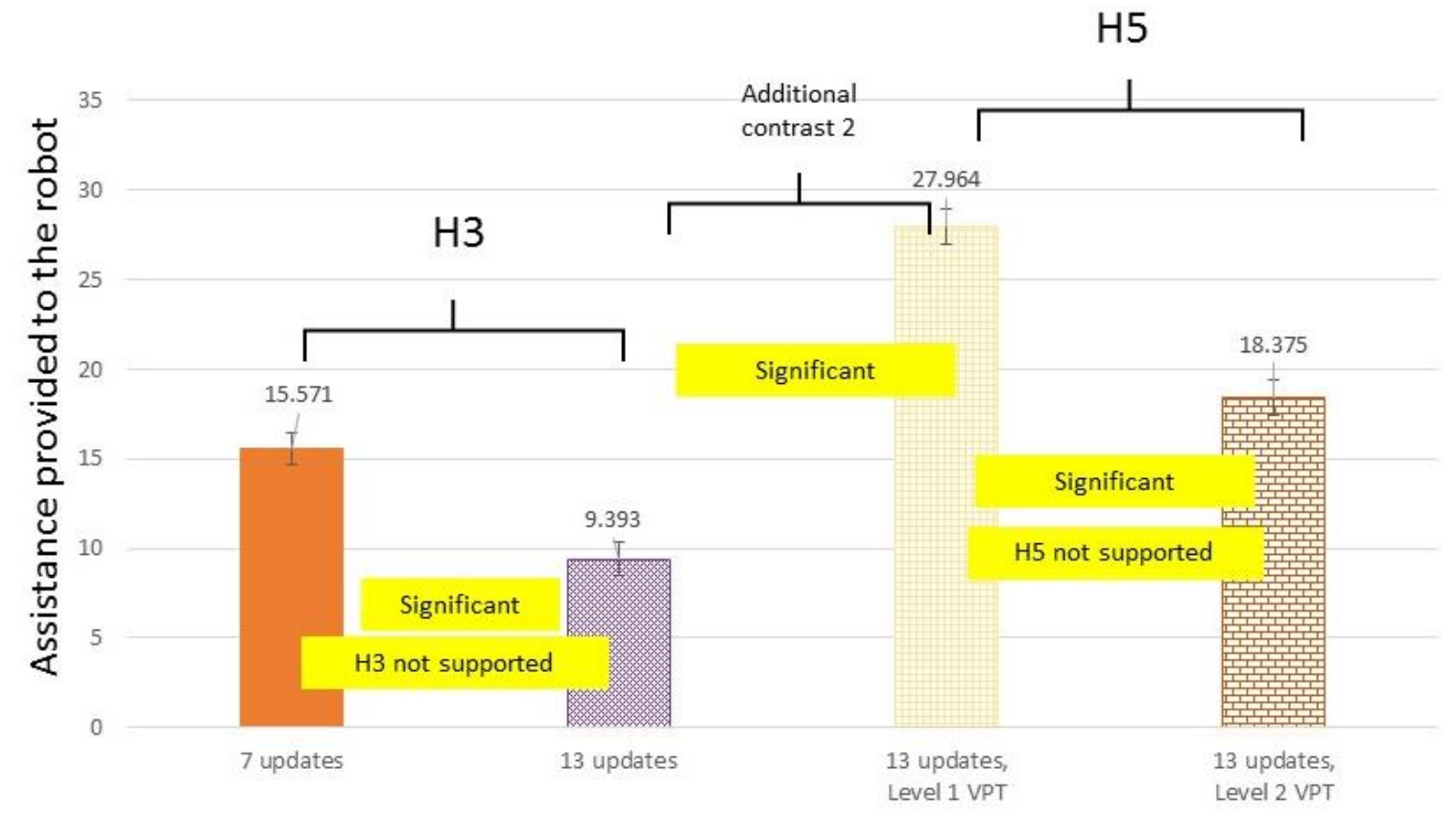

Figure 27. Pattern of mean scores on the measure of providing assistance to the robot across within-subjects conditions and planned contrasts.

\section{$\underline{\text { Additional analyses: Performance on change detection task }}$}

The future vision of Soldier-robot teams in one in which the robot and the Soldier complete separate, but interdependent tasks. If the robot's information exchanges hinder the Soldier's ability to perform his/her individual activities, then the practical utility of robot-to-human information exchanges will be diminished. As such, additional analyses were conducted to examine the effects of robot-to-human information exchanges and Level of VPT on dual task performance in the form of a change detection task. 
A one-way repeated measures ANOVA with scores on the measure of change detection performance as the within subjects variable was used to test for significant differences in mean scores on the measure of dual task performance (i.e., the change detection task) across within-subjects study conditions. There was a significant effect for within-subjects condition, Wilks Lambda $=0.470, F(3,51)=19.202, p<.001$, partial eta squared $=.530$, see table 12 and Figure 28 for mean scores on the change detection task across study conditions.

Post-hoc comparisons showed that there were significant differences in scores on the change detection task between the 7UP condition $(M=38.296)$ and the 13UP condition $(M=30.463), p<.001$. There also were significant differences in change detection scores between the 13UP condition, $(M=30.463)$, and the 13UPLevel1 condition, $(M=33.093), p=.008$. Significant differences in scores on the change detection task were not found between the 13UPLevel1 condition, $(M=33.093)$, and the 13UPLevel2 condition, $(M=34.093), p=.260$.

Table 12. Table of means for scores on the change the detection task across study conditions.

\begin{tabular}{llccc}
\hline Mission & Condition & $\begin{array}{c}\text { Change } \\
\text { detection } \\
\text { Mean }\end{array}$ & Std. Error & N \\
\hline Mission 1 & 7UP & 38.926 & 1.014 & 54 \\
Mission 2 & 13UP & 30.463 & 1.146 & 54 \\
Mission 3 & 13UPLevel1 & 33.093 & 1.226 & 54 \\
Mission 4 & 13UPLevel2 & 34.093 & 1.058 & 54 \\
\hline
\end{tabular}


Posthoc 1

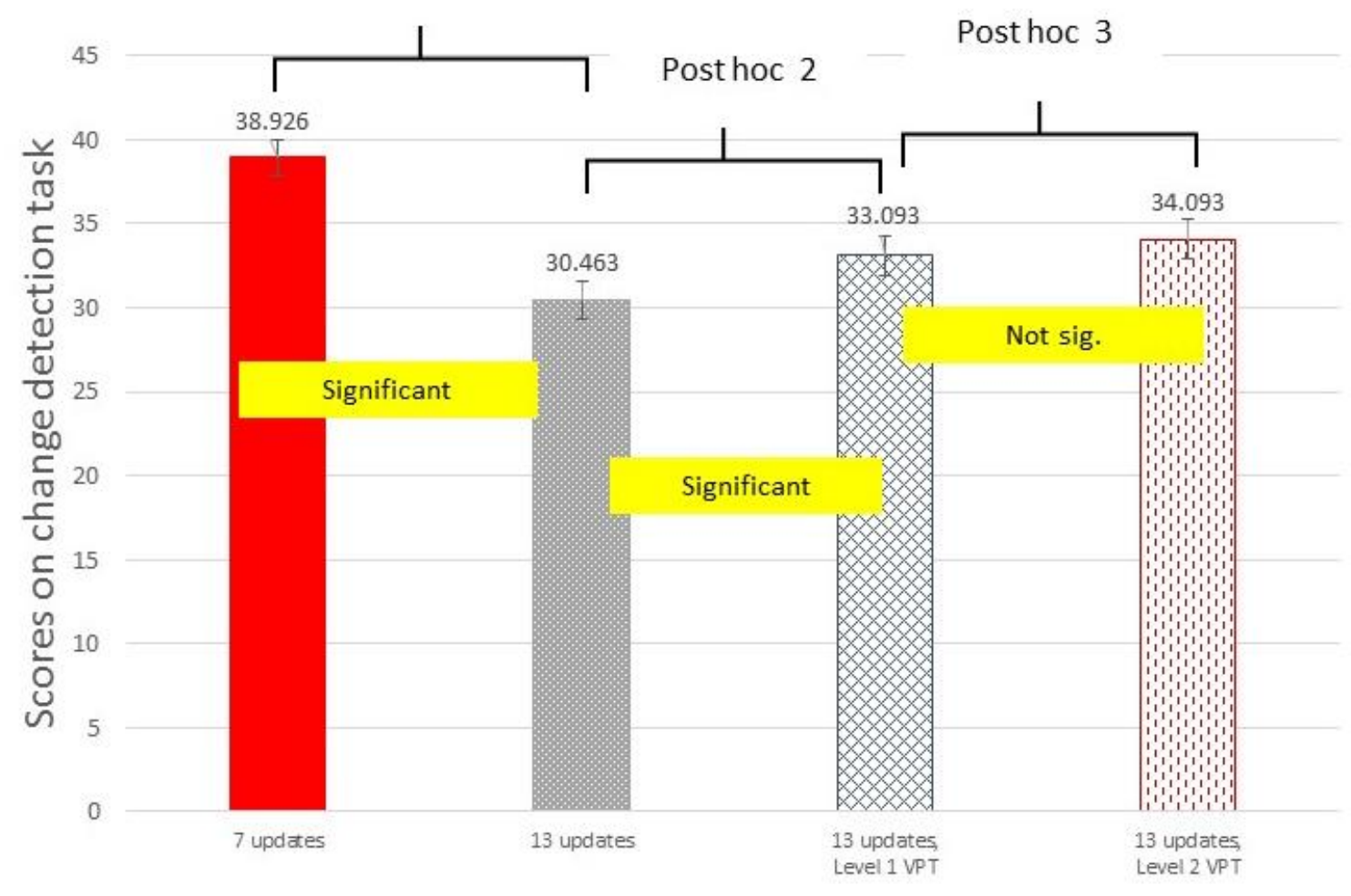

Figure 28. Pattern of mean scores and post-hoc comparisons on the change detection task across within-subjects conditions and planned contrasts.

Finally, an additional analysis of the change detection scores was conducted to compare participants' change detection performance in the training phase of the study (i.e., baseline) and participants' performance on the change detection task while completing each of the study conditions. The comparison of scores across all five (i.e., in practice and in the four study conditions) instances in which participants' completed the change detection task was isolated to a one minute interval. For the practice task, participant scores in the first minute of change detection performance were used in the 
analysis. For the remaining four study conditions, participant scores for the first minute beginning after the 30 second mark were used in the analysis. These intervals were chosen for two reasons. The first is that participants only completed the change detection task for 1 minute and 30 seconds in the practice task, while they completed the change detection task for five minutes in the study conditions. As such, the five instances in which participants completed the change detection task were not directly comparable initially. Second, in the four study conditions, participants did not begin receiving updates from the robot until 30 seconds had passed in each scenario. A goal of this analysis was to compare participant performance on the task in isolation to when participants were dual tasking by receiving updates from the robot.

A one-way repeated measures ANOVA with scores on the measure of change detection task performance as the within subjects variable was used to test for significant differences in performance on the change detection task across within-subjects conditions and in comparison to the practice change detection task. There was a significant effect for conditions, Wilks Lambda $=0.604, F(4,53)=8.702, p<.001$, partial eta squared $=.396$, see table 13 . 
Table 13. Table of mean scores on the change detection task for one minute of performance across study conditions and the practice task

\begin{tabular}{llccc}
\hline Mission & Condition & $\begin{array}{c}\text { Change } \\
\text { detection } \\
\text { Mean }\end{array}$ & Std. Error & N \\
\hline Practice & No updates & 7.72 & 0.327 & 57 \\
Mission 1 & 7UP & 7.40 & 0.268 & 57 \\
Mission 2 & 13UP & 5.81 & 0.298 & 57 \\
Mission 3 & 13UPLevel1 & 6.63 & 0.330 & 57 \\
Mission 4 & 13UPLevel2 & 5.93 & 0.350 & 57 \\
& & & & 57 \\
\hline
\end{tabular}

Post-hoc comparisons were conducted to compare participant performance on the change detection task in the practice task to their change detection performance in each of the study conditions. The post-hoc comparisons revealed that the difference in mean change detection scores between the practice task $(M=7.72)$ and the 7UP condition $(M=$ 7.40) was not statistically significant, $p=.422$. The differences in mean scores between the practice task $(M=7.72)$ and the 13UP condition $(M=5.81)$, the practice task and the 13UPLevel 1 condition $(M=6.63)$, and the practice task and the 13UPLevel 2 condition $(M=5.93)$ were all statistically significant, $p<.001, p=.012$, and $p<.001$ respectively. 


\section{CHAPTER FIVE: DISCUSSION}

\section{Summary}

The current research examined the impact of number of robot-to-human information exchanges and level of VPT context added to robot-to-human information exchanges on the development of SA regarding the robot, and the ability to use SA regarding the robot to provide assistance to the robot when necessary. Much of the prior work on human-robot teaming has focused on developing SA regarding robots by improving displays, interfaces, and other continuous monitoring devices, which require human team members to be "head's down" when interacting with robots. This is a problem, as the vision of near-future human-robot teams suggests the removal of continuous monitoring of the robot through devices and displays. As such, this study addressed deficiencies in other research studies, by investigating alternative ways to support human SA regarding the robot, and human assistance provided to the robot, when the ability to continuously monitor the robot is removed.

It was hypothesized that more robot-to-human information exchanges, presented in the form of periodic status updates from the robot, would be associated with higher levels of SA and better assistance provided to the robot than fewer robot-to-human information exchanges. These hypotheses were not supported, as the results showed that the condition with the fewest robot-to-human information exchanges resulted the highest SA scores and comparable scores on the measures of assistance provided to the robot, as 
conditions with more robot-to-human information exchanges. Also, it was hypothesized that adding levels of VPT information to robot-to-human information exchanges could support differing levels of SA regarding the robot. Such that, Level 2VPT information would support higher levels of SA, as well as better human assistance provided to the robot, than Level 1 VPT. These hypotheses also were not supported. However, the additional of Level 1 VPT did improve participants' ability to provide assistance to the robot, and seemed to mitigate some of the performance decrements associated with receiving too many updates from the robot. Finally, it was hypothesized that SA regarding the robot would be necessary for providing valuable assistance to the robot, in the event that the robot encountered difficulty completing its own tasks. This hypothesis was supported.

\section{Hypothesis H1}

Hypothesis H1 stated that higher levels of SA would be positively associated with superior human assistance provided to the robot. The result of the test of Hypothesis 1 demonstrated that SA regarding the robot is a strong predictor of providing better assistance to the robot when the robot is having trouble executing tasks as planned. This finding supports the idea that if robots can be designed in ways that promote a human's awareness of when interventions in robot tasks are needed, humans will be better able to provide those interventions and ultimately ensure the success of the team. When participant SA regarding the robot was high, participants were much better at providing 
assistance to the robot, than when participant SA was low. On average, participants with high SA scores across study conditions, scored $25 \%$ better on the measure of assistance provided to the robot, than participants with low SA scores. Not only were participants who scored high on the measure of SA, able to provide better assistance to the robot than participants who scored low on the measure of SA, but high SA participants also were roughly $30 \%$ more likely to recognize that the robot needed assistance. Taken together, these are important findings as they suggests that, at a minimum, robots that work in teams with humans should support their human partners' awareness regarding progress and status, so that humans can use that awareness to make appropriate interventions in robot tasks when needed.

These findings also have practical implications for fielding robots that do not (yet) perfectly replicate human capabilities. Specifically, human-level capabilities or full human emulation may not be essential to support teams of humans and robots completing tasks together. It is very likely that robots in the near term will not possess the metacognitive capabilities necessary to know when they are performing poorly or when to ask for human help. However, these results have helped to illustrate that if robots in the near term are able to provide status information that assists humans in knowing when robots are not performing well, then humans will be better prepared to provide the assistance that robots need, and ultimately help the team to continue on their mission. 


\section{Hypotheses $\mathrm{H} 2$ and $\mathrm{H} 3$}

The specifics regarding how to facilitate awareness regarding the robot, and assistance to the robot are more nuanced, however. Hypotheses $\mathrm{H} 2$ and $\mathrm{H} 3$ posited that more robot-to-human information exchanges would be associated with higher levels of SA, and better assistance provided to the robot than fewer robot-to-human information exchanges. Neither of these hypotheses were supported, as the pattern of mean scores on the measures of SA, and on the measures of assistance provided to the robot, trended in the opposite of the hypothesized directions. Mean scores indicated that, more robot-tohuman information exchanges were associated with lower scores on the measure of SA regarding the robot, and lower scores on the measure of human assistance provided to the robot, than fewer robot-to-human information exchanges. These findings may be due to increasing demands placed upon the human, by receiving updates from the robot too often. A look at participant performance on the change detection task revealed that the increase in the number of updates sent by the robot between the 7UP condition and the 13UP condition, not only decreased scores on the measure of SA regarding the robot, and measure of assistance provided to the robot, but also dual task performance on the change detection task, by an amount that was statistically significant.

One possible explanation for this finding is that each of the four experimental missions was scripted to last approximately 5 minutes in duration. In the 7UP condition, participants received robot-to-human information exchanges in the form of status updates 
7 times, with approximately one update every 45 seconds, beginning after the 30 second mark. The 13UP condition was also 5 minutes in duration, but participants received waypoint status updates 13 times, with approximately one update every 22-24 seconds, beginning after the 30 second mark. Thus, when the number of updates in each mission was increased, the interval between updates was reduced in half. It is possible that participants were having trouble attending to the information provided to them by the robot, or dealing with the frequency of the waypoint updates, as well as maintaining performance on the other tasks.

Although participants' scores on the measures of perceived mental workload were not statistically significantly different between the two conditions, there is prior evidence to suggest that individuals have difficulty making subjective assessments of performance when dual tasking (Horrey, Lesch, and Garabet, 2009). These findings could serve as a possible explanation for why differences on the subjective workload measure were not observed, even though significantly lower scores on SA regarding the robot, providing assistance to the robot, and the change detection task were exhibited between the 7UP condition and the 13UP condition.

Also, building SA regarding the robot required participants to use the information contained in the information exchanges to locate the robot's position on the map of the environment. Doubling the number of waypoint updates required participants, at the very least, to attend to the overhead map twice as many times, which would have required 
participants to take eyes off of the change detection task, and the OCU which displayed the robot-to-human information exchanges, in twice as many instances. Thus, simply having "eyes off" change detection task and the robot-to-human information exchanges could also help to explain drops in performance.

However, while the requirement to take "eyes off" was not ideal for maintaining high levels of performance in this study, it does simulate some of the elements of what Soldiers will be required to do when teaming with robots in field environments in the near future. Meaning that, when robots are fielded alongside Soldiers, Soldiers will likely need to periodically divert attention away from their immediate task(s), and dedicate attention to the robot. It seems, however, that the frequency or number of updates simulated in the 13UP condition may have increased demands so much so, that individuals had a difficult time attending to the robot while maintaining performance on other tasks, which is not ideal.

\section{$\underline{\text { Hypotheses } \mathrm{H} 4 \text { and } \mathrm{H} 5}$}

Hypotheses H4 and H5 stated that higher levels of VPT context added to robot-tohuman information exchanges would be associated with higher levels of SA and better assistance provided to the robot, than lower levels of VPT context. Neither of these Hypotheses were supported. The pattern of mean scores on these measures revealed that higher levels of VPT context added to robot-to-human information exchanges resulted in statistically significantly lower scores on the measures of SA, and measures of assistance 
provided to the robot than lower levels of VPT context. It was not found that Level 2 VPT context would aid participants in developing higher levels of SA, and providing better assistance to the robot by providing extra spatial processing, as originally hypothesized. However, adding Level 1 VPT context seemed to aid participants in providing better assistance to the robot than providing status updates that contained no VPT contextual information, and resulted in statistically significantly better performance on the change detection task, when compared to the 13UP condition.

There are a few reasons that might help to explain these findings. The first is related to the potential benefit of practice in completing the experimental tasks. It seemed that participants may have needed more practice in order to do well interpreting the clock face spatial reference frames provided in the Level 2 VPT condition (i.e., the 13UPLevel2 condition). In fact, if participants completed the 13UPLevel2 condition (i.e., the clock face reference frame condition) as the last mission (i.e., the $4^{\text {th }}$ trial), their SA scores for that mission were higher than if they completed the 13UPLevel 2 condition in any other trial during the course of the study. Conversely, if participants completed the 13UPLevel2 condition in the first trial, SA scores for that condition were lower than if they completed the condition in any other trial during the course of the study. Meaning, participants had the highest SA scores if the 13UPLevel2 condition was completed last, and the lowest SA scores if the 13UPLevel2 condition was completed first, throughout the course of the study. It is possible that practice helped participants to develop automaticity for some elements of the experimental tasks or aided participants in 
developing better strategies for completing tasks, which may have helped them free up any additional resources that were needed to fully understand or make use of the clock face reference frames for developing SA.

If participants did not complete the 13UPLevel2 condition in the first or last trial, they may have had difficulty switching reference frames in consecutive trials. Research by Kelly and McNamara (2010) suggested that spatial reference frames used during early exposure to an environment, can provide a framework for organizing spatial information about that environment later.

"Reference frames selected to represent one spatial layout can influence the reference frames selected to represent a second spatial layout.” (p.415)...After establishing a stable environmental reference frame, newly experienced locations can then be added into the existing reference frame." (p.419).

In the current study, there were several order of completion conditions in which participants were asked to use one reference frame to understand where the robot was located in the environment, immediately followed by the use of a different reference frame (e.g., completing the Level 1 VPT condition followed by the Level 2 VPT condition or vice versa). Participants may have had difficulty holding these different reference frames in memory and switching between them. Figures 31 and 32 depict the overhead map of the environment for the Level 1 VPT condition (13UPLevel1 condition) 
and the Level 2 VPT condition (13UPLevel2 condition) and the reference frames provided for each mission, depicted in the compass rose.

A look at mean scores on the measures of SA revealed that if participants completed the Level 1 VPT condition followed by the Level 2 VPT condition (i.e., 13UPLevel1 followed by 13UPLevel2) they had lower SA scores than if they completed the Level 2 VPT condition after any other condition. The reverse was true also. If participants completed the Level 2 VPT condition followed by the Level 1 VPT condition (i.e., 13UPLevel2 followed by 13UPLevel1), they had lower SA scores than if they completed the Level 1 condition after any other condition. The same pattern of results held true for scores on the measure of assistance provided to the robot, as well. If participants completed the Level 2 VPT condition immediately after the Level 1 VPT condition or vice versa, they had lower scores on the measure of assistance provided to the robot, than if they completed the condition after any other condition in the study.

Research by Carlson (1999) suggested that there are instances in which given multiple, active reference frames, people will chose one while inhibiting another. However, when asked to make subsequent use of the inhibited reference frame, people have trouble doing so. These findings seem to suggest that participants may have organized their understanding of information about the robot's environment and its location within that environment around one type of reference frame, and had difficulty 
when asked to switch to a different reference frame in the subsequent experimental mission $^{6}$.

Thus, for future Soldier-robot teams it may be advisable to stick with one type of reference frame when learning or reporting on the spatial layout of an operational environment. For instance, when the robot reports on spatial information about the operational environment, it may be best to be consistent with the reference frames used in pre-operational intelligence briefings and mission preparation materials.

${ }^{6}$ It should be noted that, order of completion conditions in which experimental mission 4 was the first or last trial, were mutually exclusive from order of completion conditions that required switching reference frames. See Figure 11 for more detail. 


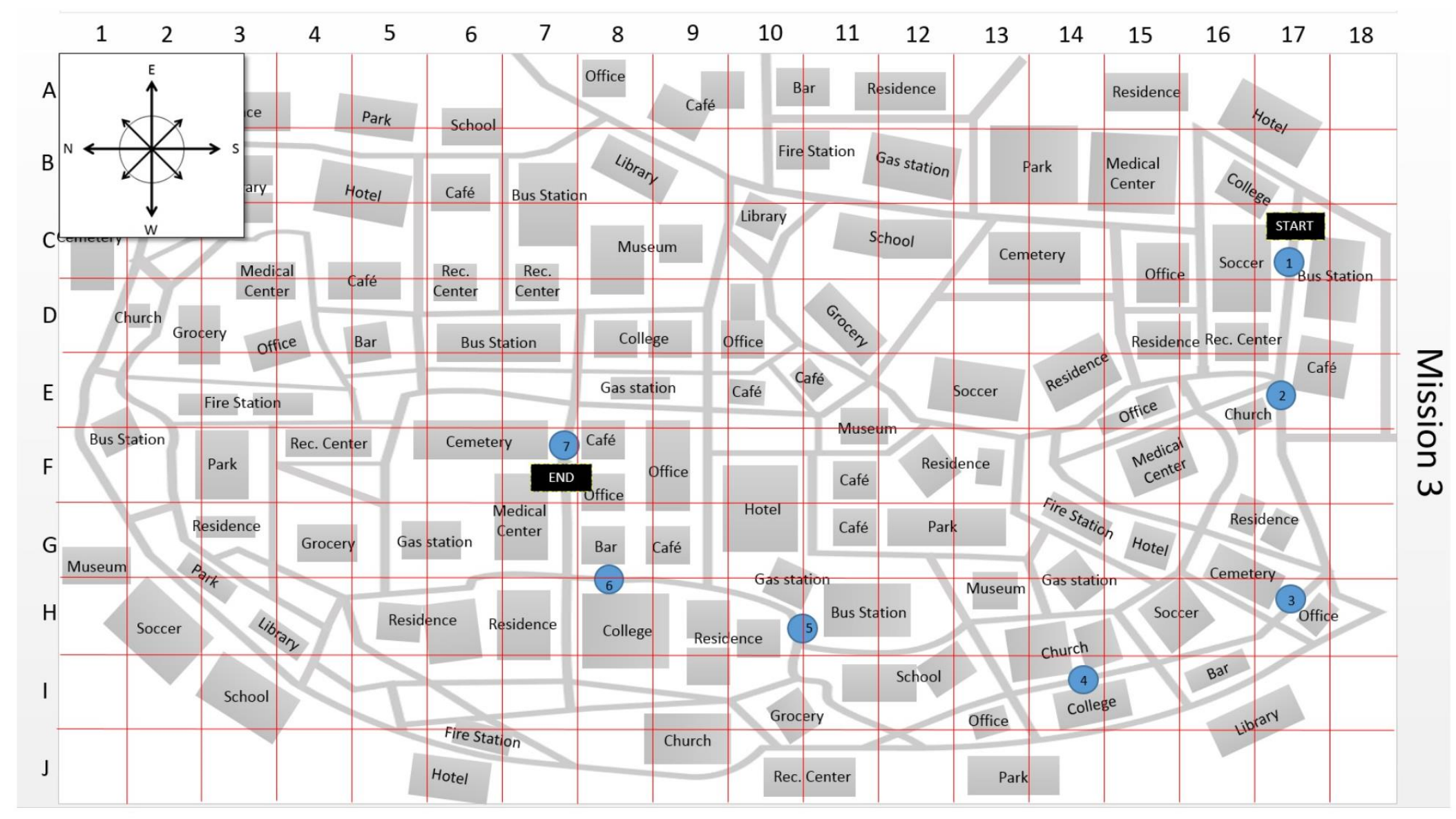

Figure 29. Overhead map provided to participants in the Traveling + waypoint status updates with Level 1 VPT context condition, experimental mission 3 . Note the compass rose contains cardinal directions only. 


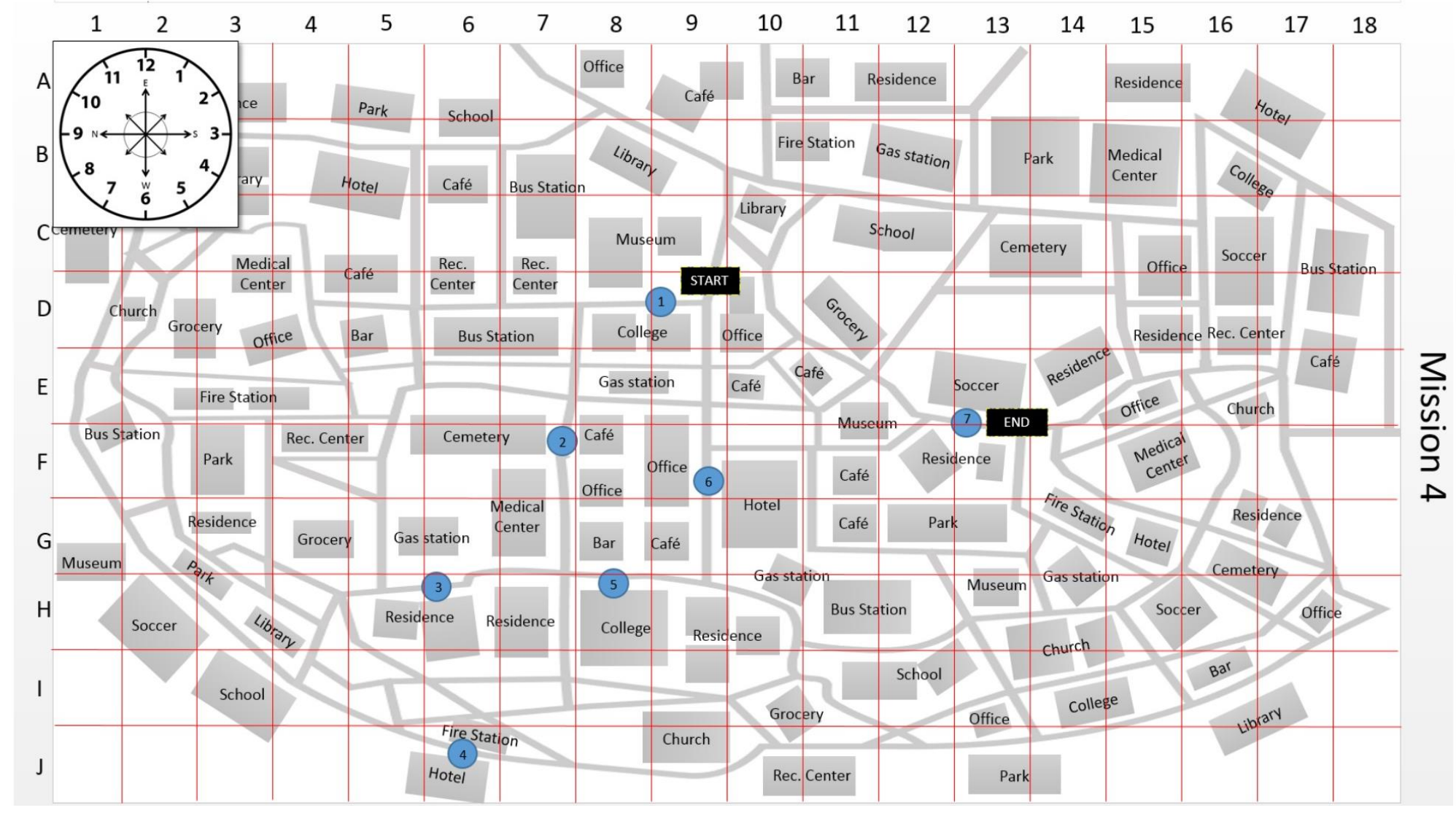

Figure 30. Overhead map provided to participants in the Traveling + waypoint status updates with Level 2 VPT context condition, experimental mission 4 . Note the compass rose contains both cardinal and clock face directions. 
Also, there is some evidence to suggest (although not conclusive) that adolescents and young adults are less likely to be familiar with analog clocks (Fendrich, 2008, Freeman; 2009; Merz, 2014; Webb, 2014), than older adults. For many adolescents and young adults, analog watches have been replaced by digital watches or devices like smartphones. Also, in many schools, there is debate surrounding whether analog clock timekeeping should be removed from educational curricula, as well as how much time should be spent covering this material, if at all (Merz, 2014). Due to the average age of the participants in this study, it is possible that the likelihood of participants being less versed in analog timekeeping is high. This could have made using reference frames that rely on familiarity with analog timekeeping more difficult, or less intuitive, as opposed to more intuitive, as originally hypothesized. It is also possible that the training materials on clock face reference frames were not enough to refresh a skill (analog timekeeping) that could have potentially gone unused by participants for a long time.

The addition of Level 1 VPT context information also seemed to benefit participants' ability to provide assistance to the robot the most. The highest scores on the measure of assistance provided to the robot was in the Traveling + waypoint status updates with Level 1 VPT context condition (13UPLevel1) condition. This is likely because Level 1 VPT context added some additional unique information about how the robot was traveling through the environment above and beyond the information provided in the waypoint status update conditions (i.e., the 7UP and 13UP conditions). Further, this additional information was presented in a format in which participants were potentially 
better able to use. The addition of Level 1 VPT information also significantly improved participants' change detection scores when compared to the 13UP condition and seemed to mitigate some of the performance decrement associated with hearing from the robot too often. Finally, although SA regarding the robot scores in this condition were low, it is possible that the items used to measure SA in this condition did not capture elements of SA that were relevant to the assistance task in this instance. Or, providing assistance to the robot in this condition may have been improved by some skill, state, or knowledge other than SA regarding the robot, and not captured in the experimental measures. .

\section{Conclusions}

\section{Implications}

While the majority of the results of the study did not turn out as originally hypothesized, the results of the tests of Hypotheses $\mathrm{H} 2$ through $\mathrm{H} 5$ provide some insight into potential best practices for facilitating situation awareness regarding the robot and assistance provided to the robot. In terms of practical utility, it is promising to note that relatively few information exchanges from the robot were needed to support the highest levels of SA in this study, the highest scores on the dual-task performance, and fairly high performance in providing assistance to the robot. The 7UP condition had the fewest robot-to-human information exchanges and was associated with the highest scores on the measure of SA, as well as the highest scores on the change detection task, and significantly higher scores on the measure of providing assistance to the robot than the 
than the 13UP condition. In fact, scores on the measure of providing assistance to the robot in the 7UP condition, were not significantly different from those in the 13UPLevel2 condition - the condition which contained the greatest amount of information concerning the robot's status and its movement through the environment. Also, the results suggest that participant performance on the change detection task in the 7UP condition was not statistically significantly different from participant performance on the change detection task during the practice/training phase of the study. Meaning that participant performance on the change detection task when dual tasking (i.e., receiving updates from the robot) was not significantly different from when participants were completing the change detection task alone. Adding periodic waypoints from the robot in the 7UP condition did not significantly detract from the participants' ability to maintain performance on a secondary task, which is a promising finding for supporting the vision of future Soldierrobot teaming.

The pattern of performance on the change detection task also may provide some insight into adapting the behavior of the robot in relation to the performance of the human. Specifically, participant performance on the change detection task may have been an indication of cognitive workload shifting between conditions. It was hypothesized that if the robot "checked in" with the human more often by providing more robot-to-human information exchanges, doing so could help the human to stay apprised of the robot's location and status. However, the opposite was found to be true, as increasing the number of waypoint updates provided by the robot resulted in a statistically significant reduction 
in performance on all of the dependent measures. However, the condition with the fewest information exchanges had the highest SA scores, change detection scores, and adequate scores on the measure of providing assistance to the robot. These findings are somewhat consistent with studies conducted with human-human teams operating under shifting workload conditions. Researchers have described that when members of high performing human-human teams shift from low workload conditions to operating under high workload conditions, they often exhibit patterns of communication marked by a reduction in information sharing, resulting in more efficient team communication overall (Johannesen, Cook, \& Woods, 1994; MacMillan, Entin, \& Serfaty, 2004; Urban, Bowers, Monday, \& Morgan, 1993). For fielding future human-robot teams, if the robot can sense that the human is having trouble dual tasking, the robot may be able to adjust the frequency with which it sends updates, and thus support the ability of the human to complete all of his/her mission tasks.

Similarly, while the addition Level 2 VPT context to robot-to-human information exchanges did not improve scores on the measure of SA regarding the robot, or the measure of assistance provided to the robot as hypothesized, the addition of Level 1 and Level 2 VPT context seemed to help participants to mitigate some of the performance decrements associated with hearing from the robot too often, (perhaps also by shifting cognitive workload). Participants' scores on the change detection task were statistically significantly better in both the 13UPLevel 1 and 13UPLevel 2 conditions than in the 13UP condition, even though the number of updates provided to the human by the robot 
was the same in all three conditions. It is possible that even though the frequency and number of updates were the same in each condition, that the traveling updates did not require as much time with "eyes off" the change detection task in the same way that the additional waypoint status updates did, which likely helped performance on the change detection task.

This is important to note because in this study, hearing from the robot roughly 3-4 times a minute seemed to be detrimental, whereas roughly 1-1.5 times a minute was more manageable in terms of dual tasking. Recent research studies (Abich, Barber, Talone, Phillips, Jentsch, Pettitt, \& Elliot, 2016; Barber, Abich, Phillips, Talone \& Jentsch 2015;Talone, Phillips, \& Jentsch, 2016) in which participants were asked to request robot status reports when they felt necessary, found that on average, participants requested reports at a frequency closer to the 3-5 times per minute as opposed to 1-2 times per minute - the frequency which resulted in the highest levels of SA in this study. As such, if more status updates from the robot are needed, or desired, by users at a greater frequency, it may be best to include periodic traveling status updates that present information with reference to global-relative directions when possible.

On the whole, these results provide some promising practical considerations for the design of information sharing between robot and human teammates in the near term. With size, weight, and computing restrictions, designers of robotic teammates intended to be deployed in the near-term, will be contending with practical tradeoffs concerning the 
inclusion of various hardware used to support robot sensing, as well as information processing onboard the machine itself. As such, it is practically important to note that our participants were able to determine from relatively few information exchanges, coupled with less contextual information, as opposed to more contextual information, the location of the robot, as well as implications associated with its surroundings. These findings could mean that designers may be able to devote less sensing hardware and computing resources to robot-to-human information exchanges than originally hypothesized. The result of which may translate into space and cost savings overall.

Also, clock face directions were chosen for this study because VPT theory supports the idea that communicating spatial information in reference to an outside observers view of the environment (Level 2 VPT) is beneficial for the observer learning the position of objects in that environment. Clock face directions are one way to communicate spatial information in a format that is observer-centric because they are relative to the (in this study) human observer's perspective of the environment, as opposed to the robot's perspective of the environment. They also represent standard terminology in military contexts. However, recent empirical evidence has shown that Soldiers may have a preference for using cardinal or global-relative terminology when communicating directional information to a robot. A study conducted by Barber, Wohleber, Parchment, Jentsch and Elliot (2014), surveyed Soldiers located at Ft. Benning, Georgia on the vocabulary they would use when commanding a robot to move through an environment. Results showed that Soldiers favored using cardinal directions 
when commanding the robot, as opposed to clock face directions when given the option to use either. It is possible that this is the case because prior experience with autonomous robotic teammates for most people (included those represented in this study) is low. Therefore, people may be assuming that robots naturally utilize advanced sensors and Global Positioning System (GPS) capabilities to naturally "think" in global relative perspectives, which may not actually be true.

It is also possible that the use of clock face directions may not have been a strong enough operationalization of Level 2 VPT construct. A look at some of the literature on perspective taking communications used in human-human teams suggests that when people communicate Level 2 information in another's point of view, they spend a large amount of their overall utterances doing so (Shelton \& McNamara, 2004). Also many utterances are spent trying to ground information before spatial information is communicated (Trafton, Cassimatis, Bugajska, Brock, Mintz, \& Schultz, 2005). In the current study, a stronger (or perhaps different) operationalization of Level 2 context added to robot-to-human information exchanges may have been needed to better support the Level 2 construct and VPT theory.

\section{Limitations of the study}

In this study, each condition was tied to a unique waypoint route through the environment for the robot. This was a decision made to reduce the effect that memorizing the robot's path may have had. As a result, it is difficult to determine if certain routes 
through environment were systematically more or less difficult than others. Although great care was taken to ensure that each route would be roughly equivalent, it is possible that one or more may have been easier or more difficult to complete. Further, only one level of treatment was tested in each unique route through the environment. Thus, it is difficult to know whether certain levels of treatment, Level 2 VPT for instance, would have been more helpful in a route other than the one tested in the Level 2 VPT condition. Although effort was given to try to ensure that this was not the case, it is possible that different route/treatment combinations could have produced different a pattern of results than the ones presented here.

Further, the modality in which information was shared with the human by the robot was not a variable of interest in this study. Although robot-to-human information exchanges were presented to participants through text exchanges, the purpose was to test whether the addition of VPT information was helpful in building and maintaining SA as well as providing assistance to the robot when necessary, and not whether the text modality was the best way to present robot-to-human information exchanges. It is very possible that presenting robot status information through different modalities or multiple modalities (e.g., speech, text, tactile, or some combination of) will show a different pattern of results.

Not including the female participants in the analyses also is a major limitation of this study. While the ratio of females and males was originally chosen to more closely 
represent the ratio of genders in military contexts, it was disheartening to see that removing the females' scores from the analyses aided in the statistical interpretability of the results (see Appendix I). This is especially discouraging because females and minorities tend to be under-represented in scientific research studies in general (Nature, 2010; Shavers-Hornaday, Lynch, Burmeister, \& Torner, 2010). Any recommendations generated from this study may not generalize to female Soldiers whom, now permitted in all types of combat operations, could benefit from research results that generalize to them.

\section{Areas for future research}

For practical purposes it may be worthwhile to test the different levels of treatment as a between-subjects study condition for each waypoint route generated for this study. Doing so would give a more clear indication of whether or not certain levels of treatment would have a differing impact on SA and assistance under different types of waypoint routes taken by the robot.

Also, the results of this study supported using global relative terms to present robot traveling information, as opposed to person relative terminology. However, it is important to note that for programs working to develop near-future robotic teammates, like the Robotics Collaborative Technology Alliance (RCTA), there is an imperative to deemphasize reliance on information gathered by the robot from networked devices and Global Positioning Systems. It is possible that although global-relative status information 
is helpful, that information may not always be available for near-future robotic teammates to utilize. It is important that future research investigate whether other types of information may be similarly beneficial. Or, if situations in which there is complete absence of global-relative position information, whether person-relevant information, like Level 2 VPT context, can act as a sufficient substitute.

In addition, the future vision of Soldier-robot teams in one in which the members of the team are dislocated from one another and completing separate tasks. This means that there will likely be instances in which the Soldier will need to move through an operational environment on their own waypoint-like path while the robot(s) is/are simultaneous navigating through the environment. In this study, the participant playing the role of the Soldier was stationary. Future research might investigate whether Level 2 VPT context represented in clock face directions may be beneficial for communicating relative positions and directions when both members of the team are moving through the environment.

Finally, females were excluded from the analyses of the study results due to large gender effects associated with spatial ability and the development of SA regarding the robot. It would be worthwhile to explore ways to support reference frames that may favor female spatial thinking. Doing so would aid in providing results and recommendations that generalize to female Soldiers. For instance, research by MacFadden, Elias, and Saucier (2003) suggested that women give directions that feature landmarks and left/right 
turns, whereas males include more cardinal and distance information when giving directions. In terms of design considerations, it may be practical to build information exchange interfaces that include custom features that allow different types of users to select reference frames for providing spatial information. This might include the robot providing directional information like "I am to the left/right of the building" vs. "I am on the North side of the building." Doing so may be important as, although VPT information did not seem to improve scores on the measures of SA regarding the robot, it did improve scores on the measure of providing assistance to the robot. 


\section{APPENDIX A: UCF APPROVAL OF HUMAN-SUBJECTS}

RESEARCH 


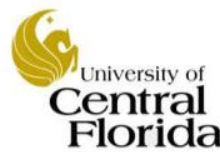

University of Central Florida Institutional Review Board Office of Research \& Commercialization

12201 Research Parkway, Suite 501

Orlando, Florida 32826-3246

Telephone: 407-823-2901 or 407-882-2276

www.research.ucf.edu/compliance/irb.html

\section{Approval of Human Research}

$\begin{array}{ll}\text { From: } & \text { UCF Institutional Rev } \\ & \text { FWA00000351, IRB0 } \\ \text { To: } & \text { Elizabeth K. Phillips } \\ \text { Date: } & \text { July 06, 2015 }\end{array}$

Dear Researcher:

On 07/06/2015, the IRB approved the following human participant research until 07/05/2016 inclusive:

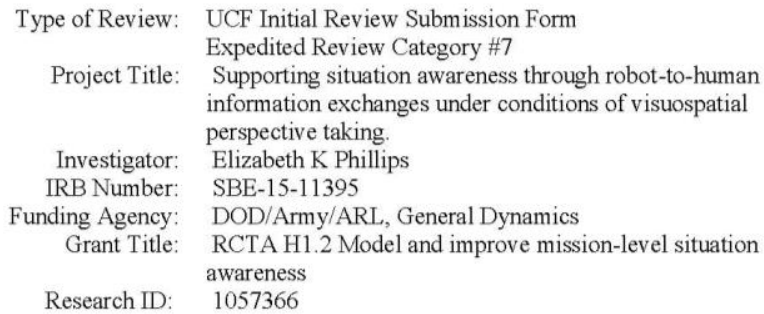

The scientific merit of the research was considered during the IRB review. The Continuing Review Application must be submitted 30days prior to the expiration date for studies that were previously expedited, and 60 days prior to the expiration date for research that was previously reviewed at a convened meeting. Do not make changes to the study (i.e., protocol, methodology, consent form, personnel, site, etc.) before obtaining IRB approval. A Modification Form cannot be used to extend the approval period of a study. All forms may be completed and submitted online at https://iris.research.ucf.edu

If continuing review approval is not granted before the expiration date of 07/05/2016, approval of this research expires on that date. When you have completed your research, please submit a Study Closure request in iRIS so that IRB records will be accurate.

Use of the approved, stamped consent document(s) is required. The new form supersedes all previous versions, which are now invalid for further use. Only approved investigators (or other approved key study personnel) may solicit consent for research participation. Participants or their representatives must receive a signed and dated copy of the consent form(s).

All data, including signed consent forms if applicable, must be retained and secured per protocol for a minimum of five years (six if HIPAA applies) past the completion of this research. Any links to the identification of participants should be maintained and secured per protocol. Additional requirements may be imposed by your funding agency, your department, or other entities. Access to data is limited to authorized individuals listed as key study personnel

In the conduct of this research, you are responsible to follow the requirements of the Investigator Manual.

On behalf of Sophia Dziegielewski, Ph.D., L.C.S.W., UCF IRB Chair, this letter is signed by:

Page 1 of 2 


\section{Goame Sunatoi}

Signature applied by Joanne Muratori on 07/06/2015 02:52:08 PM EDT

IRB manager

Page 2 of 2 
APPENDIX B: U.S. ARMY HUMAN RESEARCH PROTECTIONS OFFICE HEADQUARTERS-LEVEL ADMINISTRATION REVIEW 


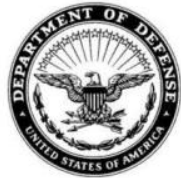

REPLYTO ATENTION OF

ARL-HRPO

\author{
DEPARTMENT OF THE ARMY \\ U.S. ARMY RESEARCH, DEVELOPMENT AND ENGINEERING COMMAND \\ ARMY RESEARCH LABORATORY \\ ABERDEEN PROVNG GROUND MD 21005-5067
}

MEMORANDUM FOR:

SUBJECT:

Protocol/Title:

Principal Investigator:

Protocol Number:
9 July 2015

1. Review Outcomes: The ARL Human Research Protections Official (HRPO) has approved the above referenced protocol. HRPO review is required for non-DoD institutions conducting research involving human subjects supported by the Department of Defense (DoD), per DoDI 3216.02, Encl 3.4.c.

\section{Requirements:}

a. Substantive Changes to the Protocol: The HRPO must review and accept the IRB's determination when substantive modifications are made to this research protocol, and any modifications that could potentially increase risk to subjects, before the changes are implemented (DoDI 3216.02, Encl 3.4.c(2)(c)). Substantive modifications include a change in principal investigator, change or addition of an institution, elimination or alteration of the consent process, change to the study population that has regulatory implications (e.g., adding children, adding active duty population, etc.), significant change in study design (i.e., would prompt additional scientific review), or a change that could increase risks to subjects.

b. Continuing Review: The HRPO must ensure an appropriate continuing review occurred within the required timeframe, (DoDI 3216.02, Encl 3.4.c(2)(d)). Submit communication from the IRB regarding any lapse in IRB approval.

c. Study Closure: The HRPO must be informed of the date and reason for study closure (i.e., study completed, insufficient enrollment to sustain the research, etc.). The HRPO must receive the final study report submitted to the IRB, including a copy of any acknowledgement documentation and any supporting documents, as soon as all documents become available. 
ARL-HRPO

SUBJECT: Human Research Protection Official (HRPO) Review

Protocol Number: ARL 15-067

d. Notification: The investigator must immediately notify the HRPO of the occurrence of any of the following (DoDI 3216.02, Encl 3.4.b(4)): different IRB.

(1) When the IRB used to review and approve the research changes to a

(2) The knowledge of any pending compliance inspection/visit by the Food and Drug Administration (FDA), Office for Human Research Protections, or other

government agency concerning this research; the issuance of inspection reports, FDA Form 483 , warning letters, or actions taken by any regulatory agencies including legal or medical actions.

(3) Suspension or termination of this research study by the IRB, the institution, the sponsor, or regulatory agency.

(4) Confirmed unanticipated problems involving risks to subjects or others related to this research study. study.

(5) Confirmed serious or continuing noncompliance related to this research

3. Caution: Do not construe this correspondence as approval for any contract funding. Only the Contracting Officer/Grants Officer can authorize expenditure of funds. Also, do not construe this as IRB approval.

4. The POC for technical questions regarding this report is Ms. Theresa Straut (410) 278-5928 or theresa.m.straut.civ@mail.mil.

\footnotetext{
STRAUT.THERE ${ }_{\substack{\text { Digitally signed by } \\ \text { SRAUTHYPSAM }}}$

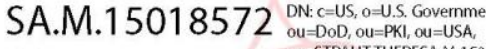

42 $\mathrm{c}=$ =STRAUT.THERESA.M. 150185724

42 on=STRAUT.THERESA.M. 1501857242
Date: 2015.07.09 12:09:05-04'00'

THERESA M. STRAUT, CIP, RAC

Human Protection Administrator, ARL
} 


\section{APPENDIX C: GRAPHS OF HYPOTHESIZED RESULTS}


Conceptual representation of the hypothesized pattern of results for scores on the measure of SA regarding the robot.

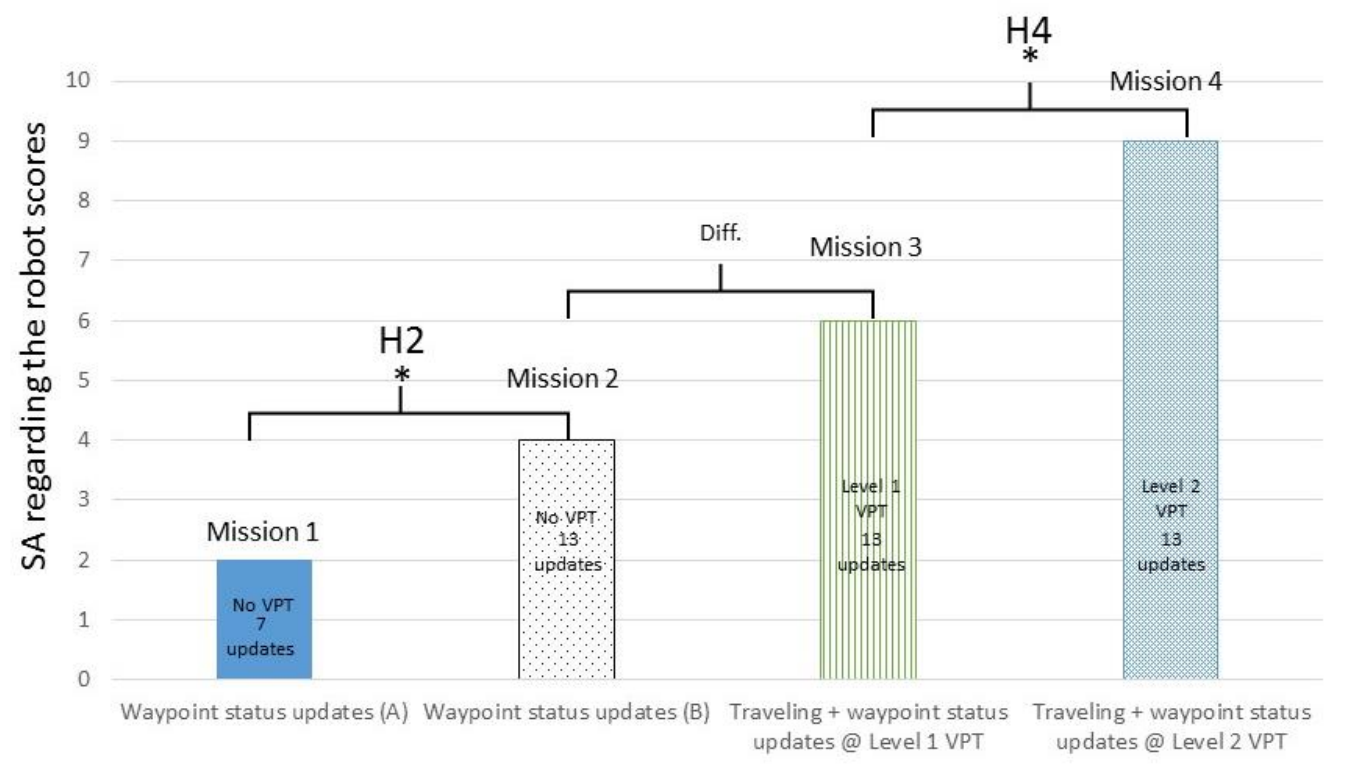


Conceptual representation of the hypothesized pattern of results for scores on the measure of human assistance provided to the robot.

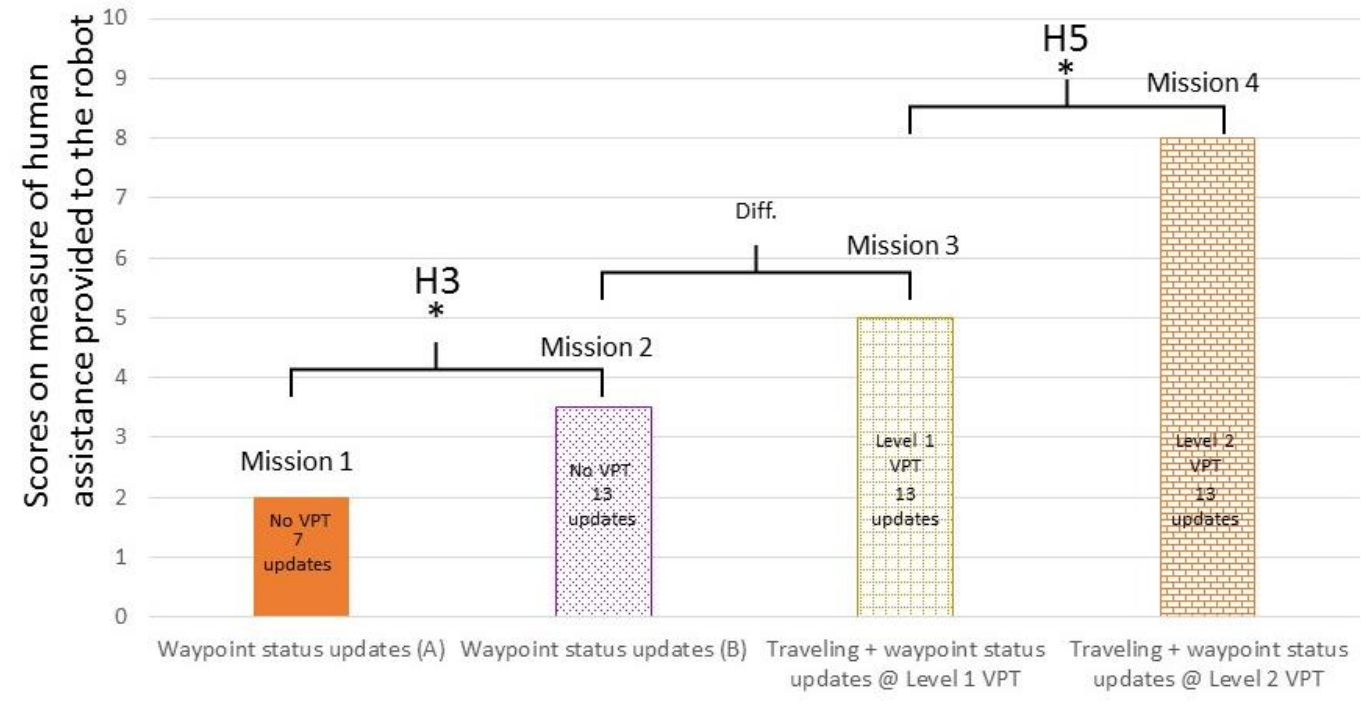


Conceptual representation of the hypothesized pattern of results for scores on the change detection task.

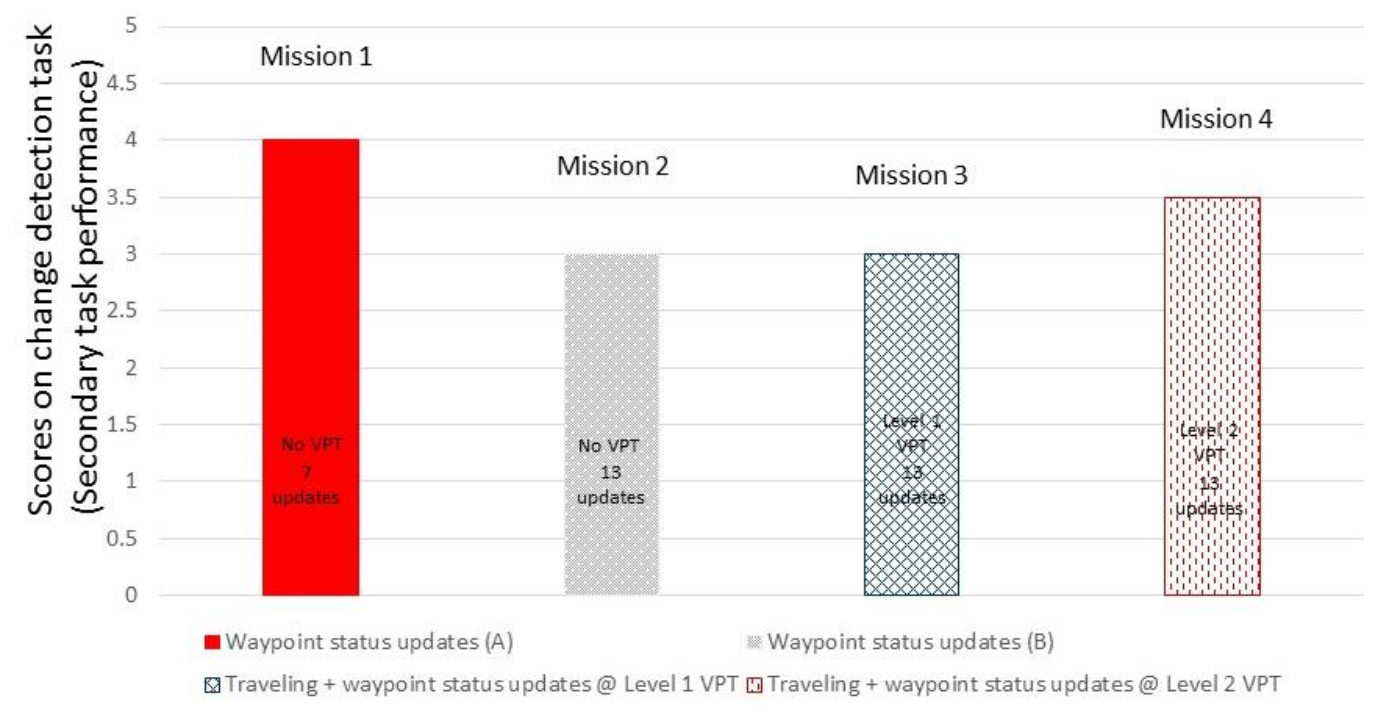


Hypothesized positive relationship between SA regarding the robot and human assistance provided to the robot.

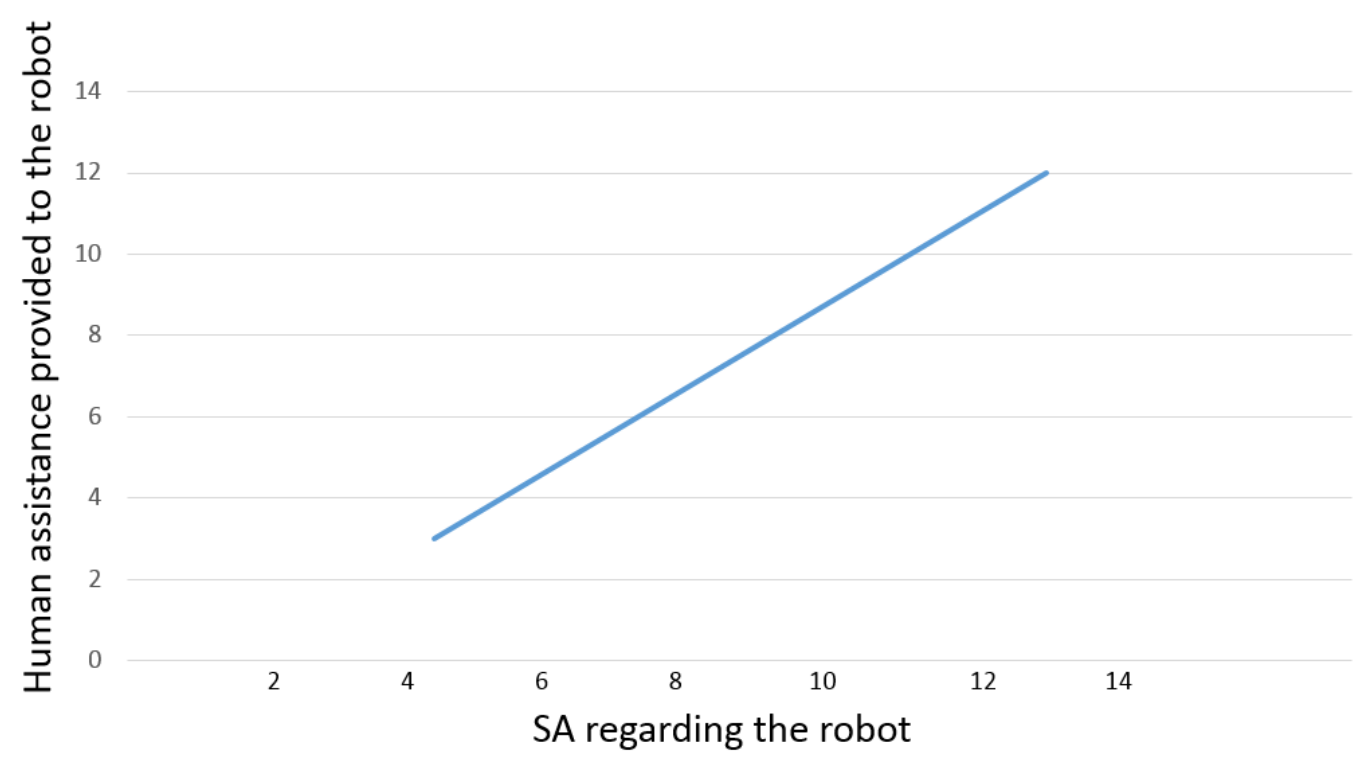




\section{APPENDIX D: GRAPHICAL REPRESENTATIONS OF EXPERIMENTAL SCENARIOS}


Key

Red Triangles $=$ Planned waypoint route

Green Circles $=$ Robot's actual waypoint route

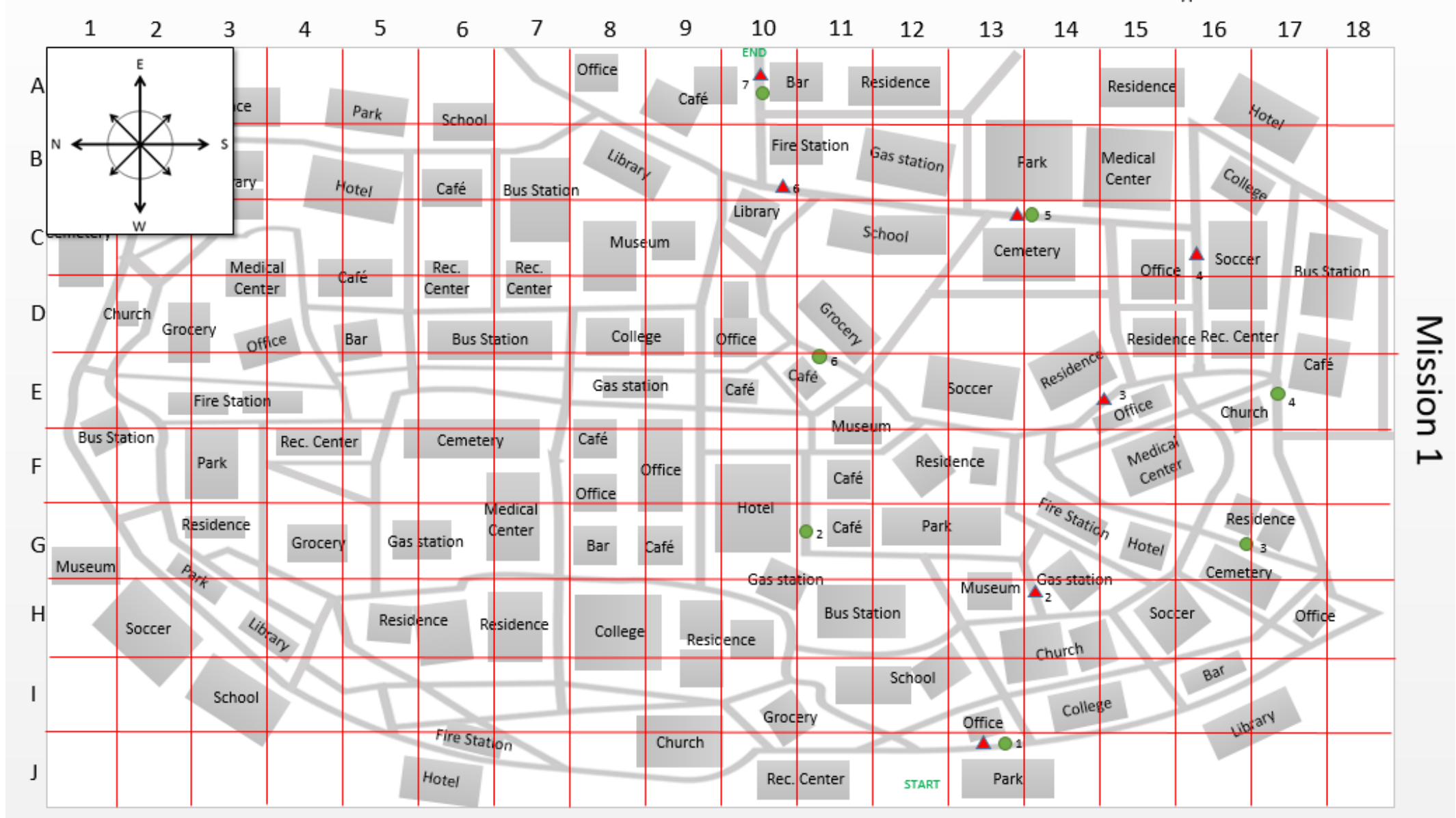


$\frac{\text { Key }}{\text { ned wayp }}$

Red Triangles $=$ Planned waypoint route

Green Circles $=$ Robot's actual waypoint route

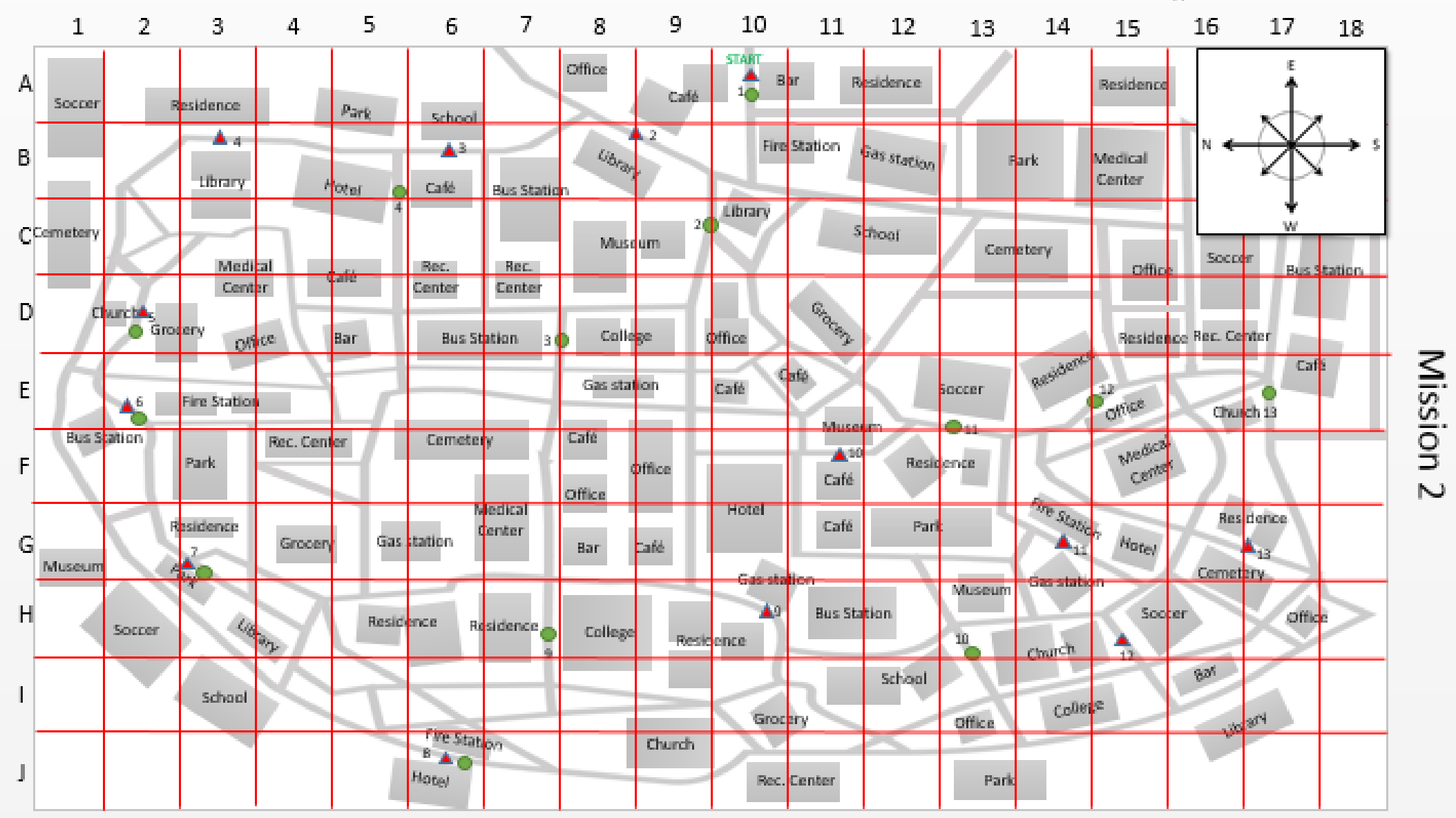


Red Triangles $=$ Planned waypoint route

Green Circles $=$ Robot's actual waypoint route

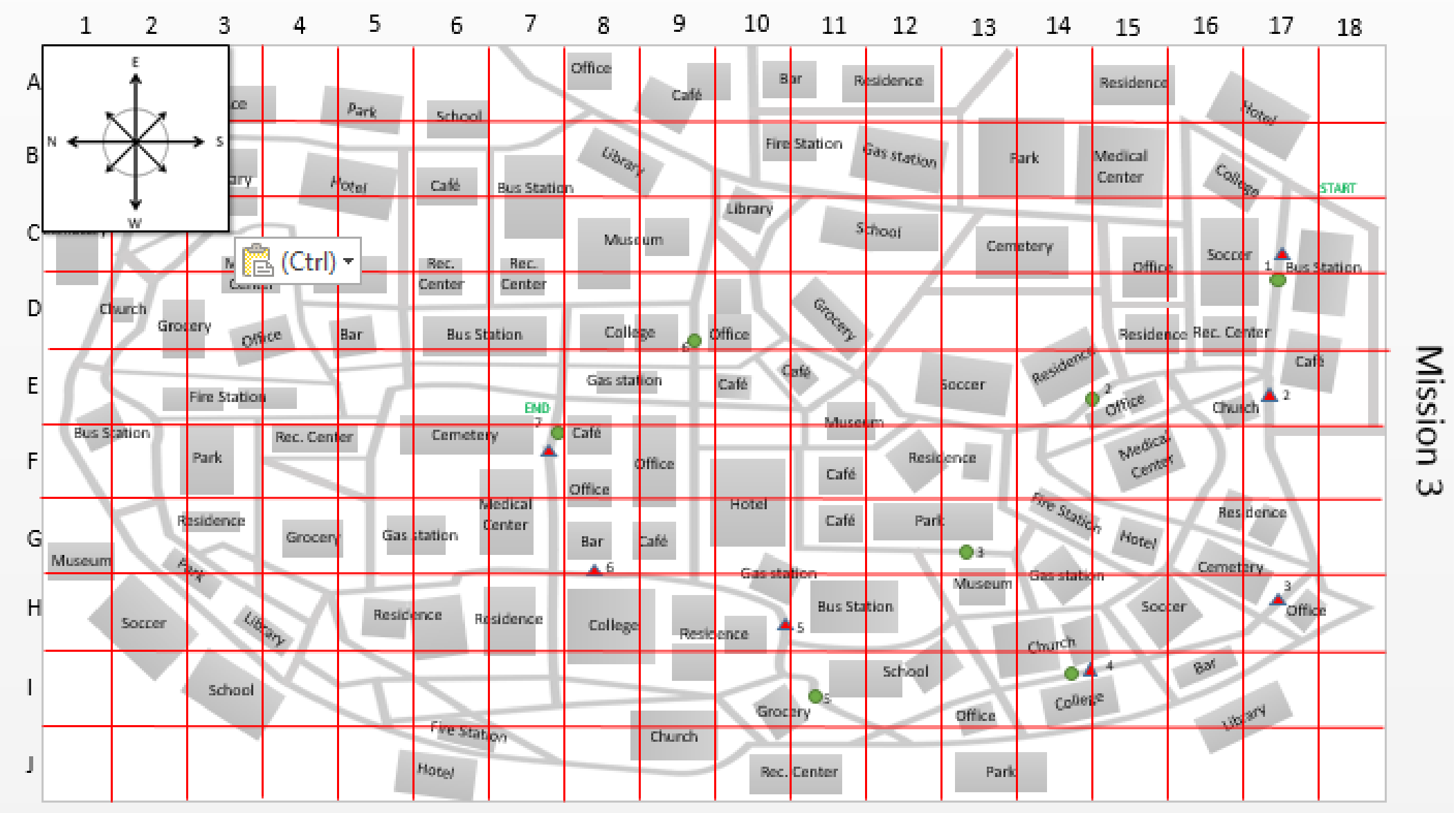


Key

Red Triangles $=$ Planned waypoint route

Green Circles = Robot's actual waypoint route

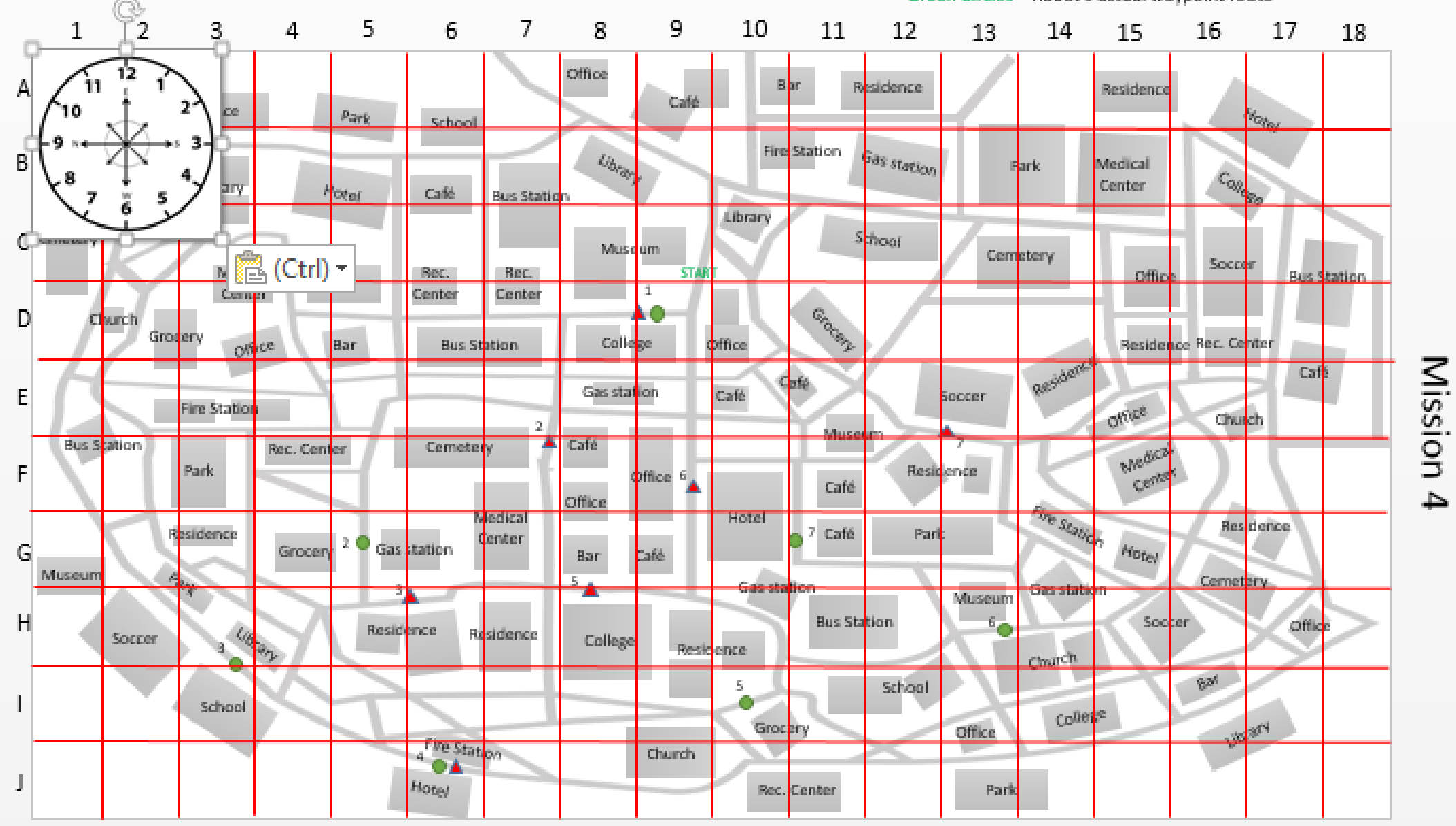


Key

Training mission 1

Red Triangles $=$ Planned Route

\author{
Training mission 1
}

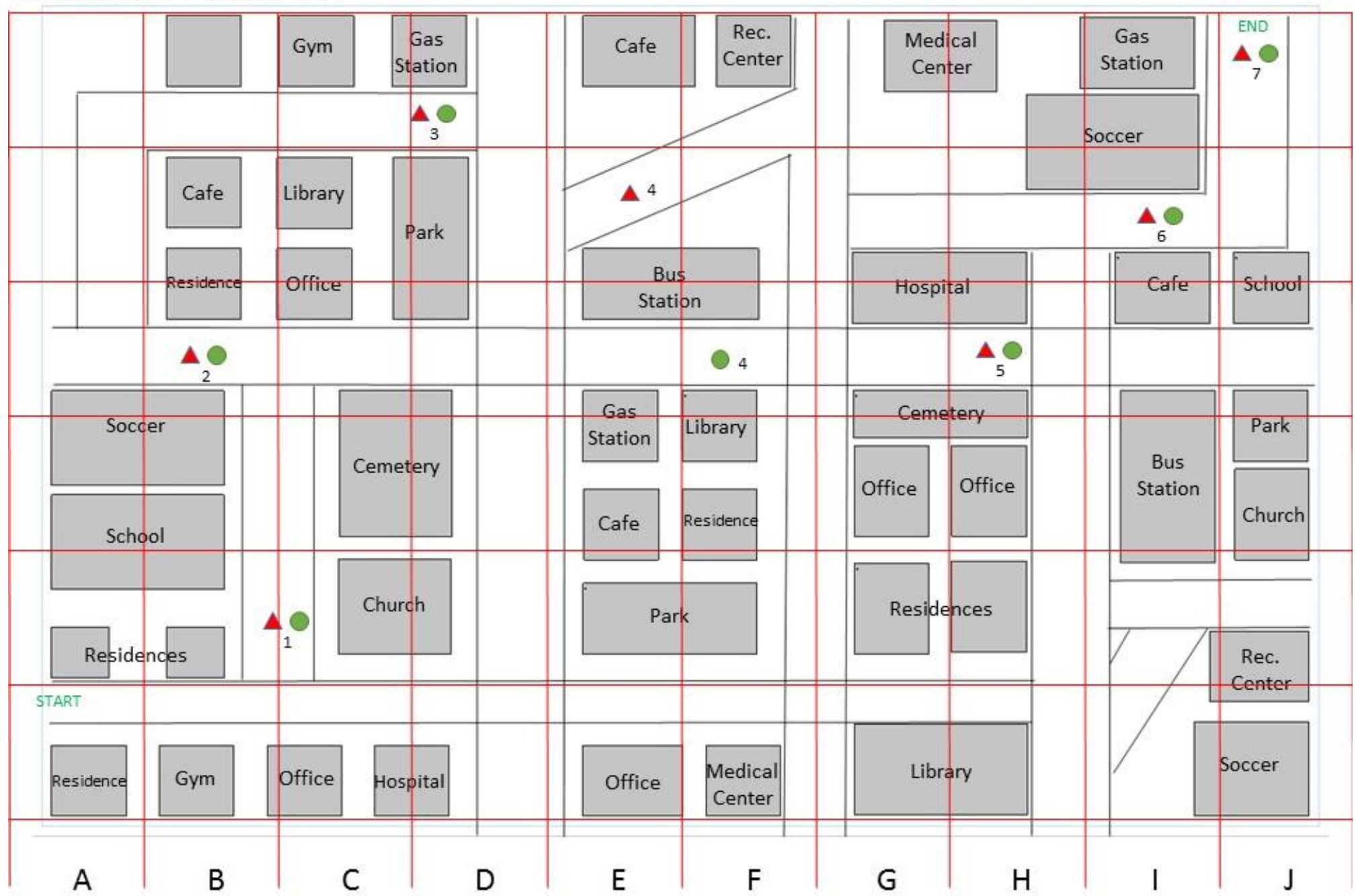


Key

Training mission 2

Red Triangles $=$ Planned Route

Green Circles = Robot's actual route

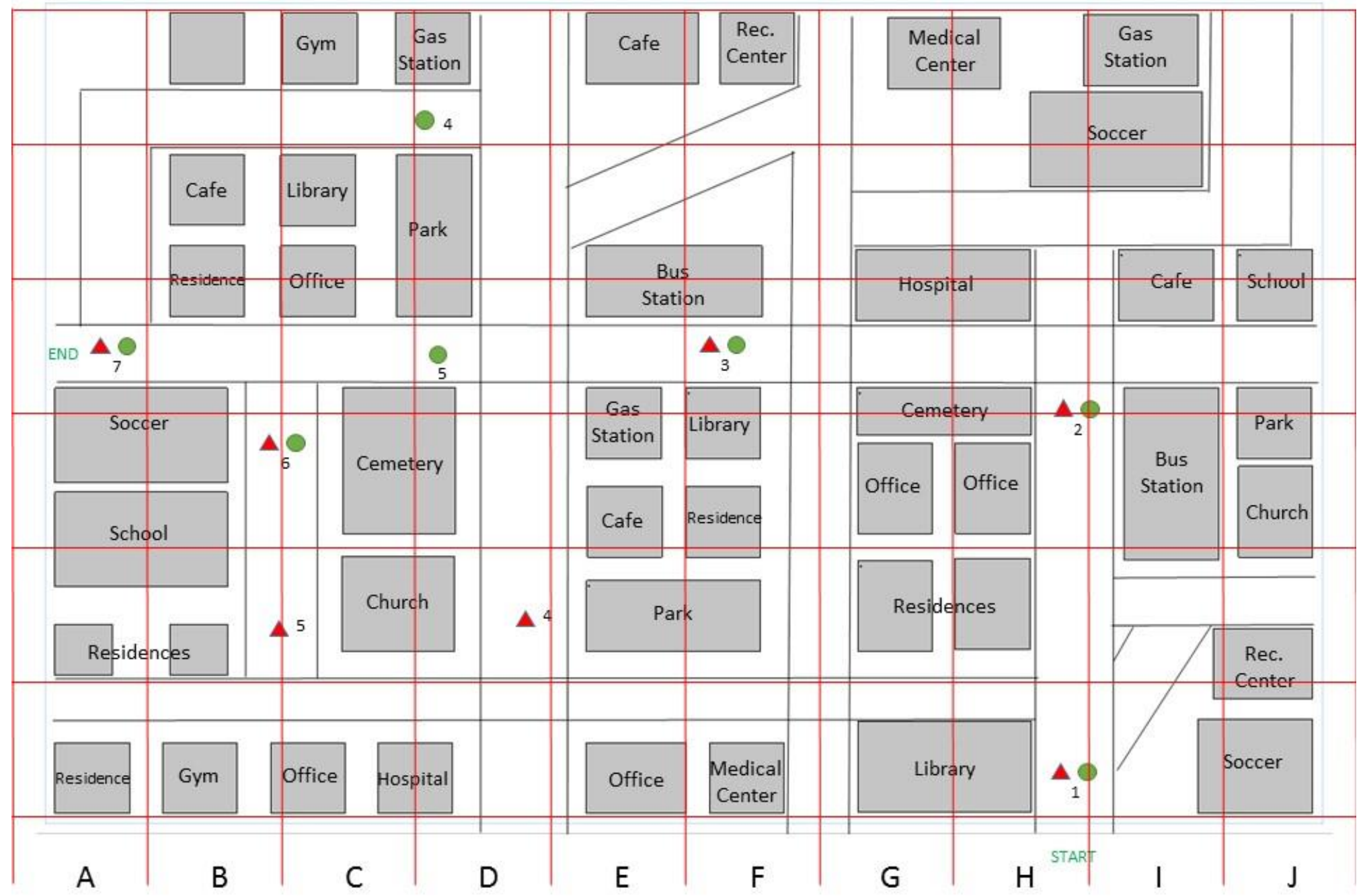


Key

Training mission 3

Red Triangles $=$ Planned Route

START

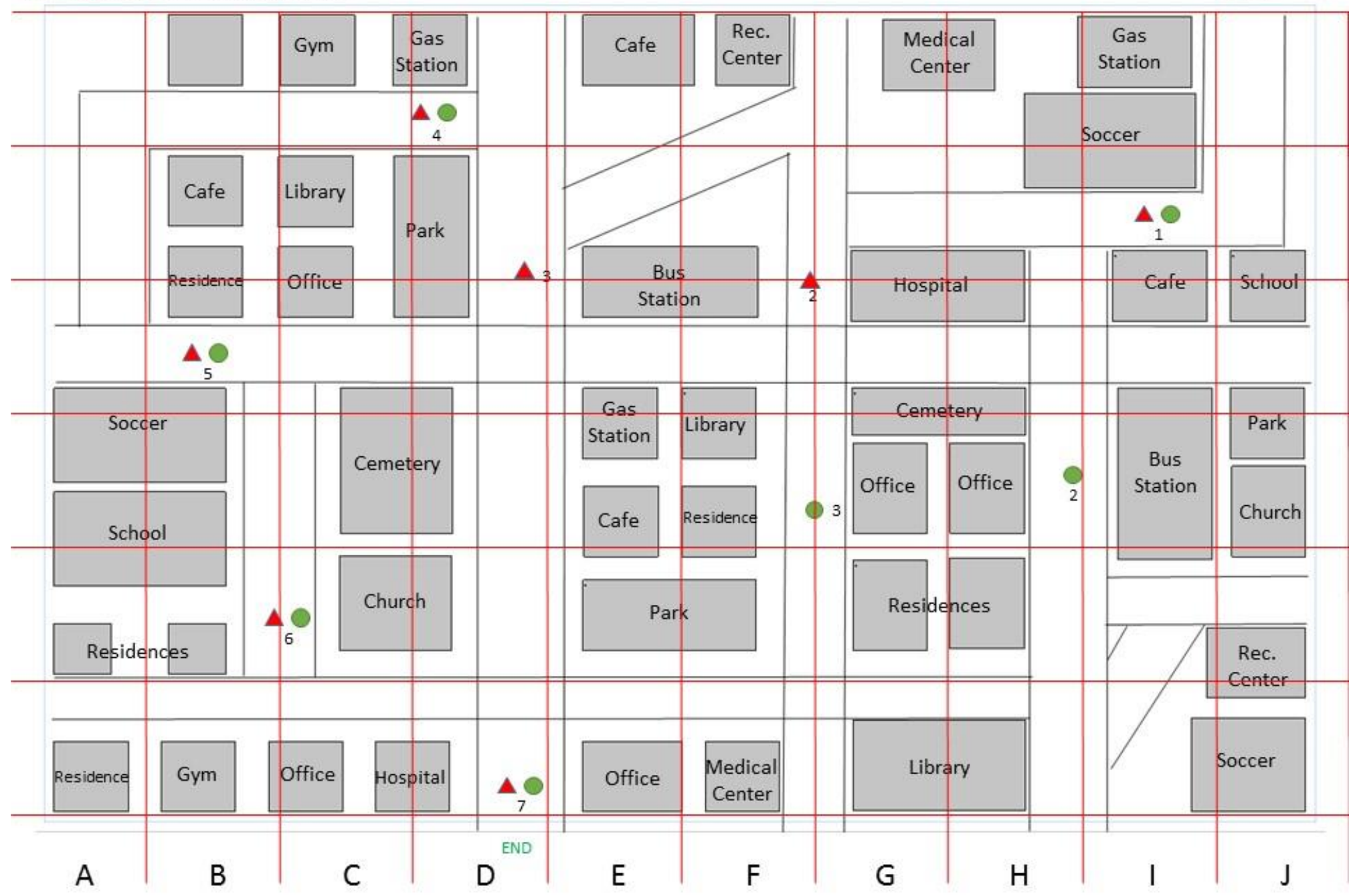


APPENDIX E: OVERHEAD MAPS PROVIDED TO PARTICIPANTS 


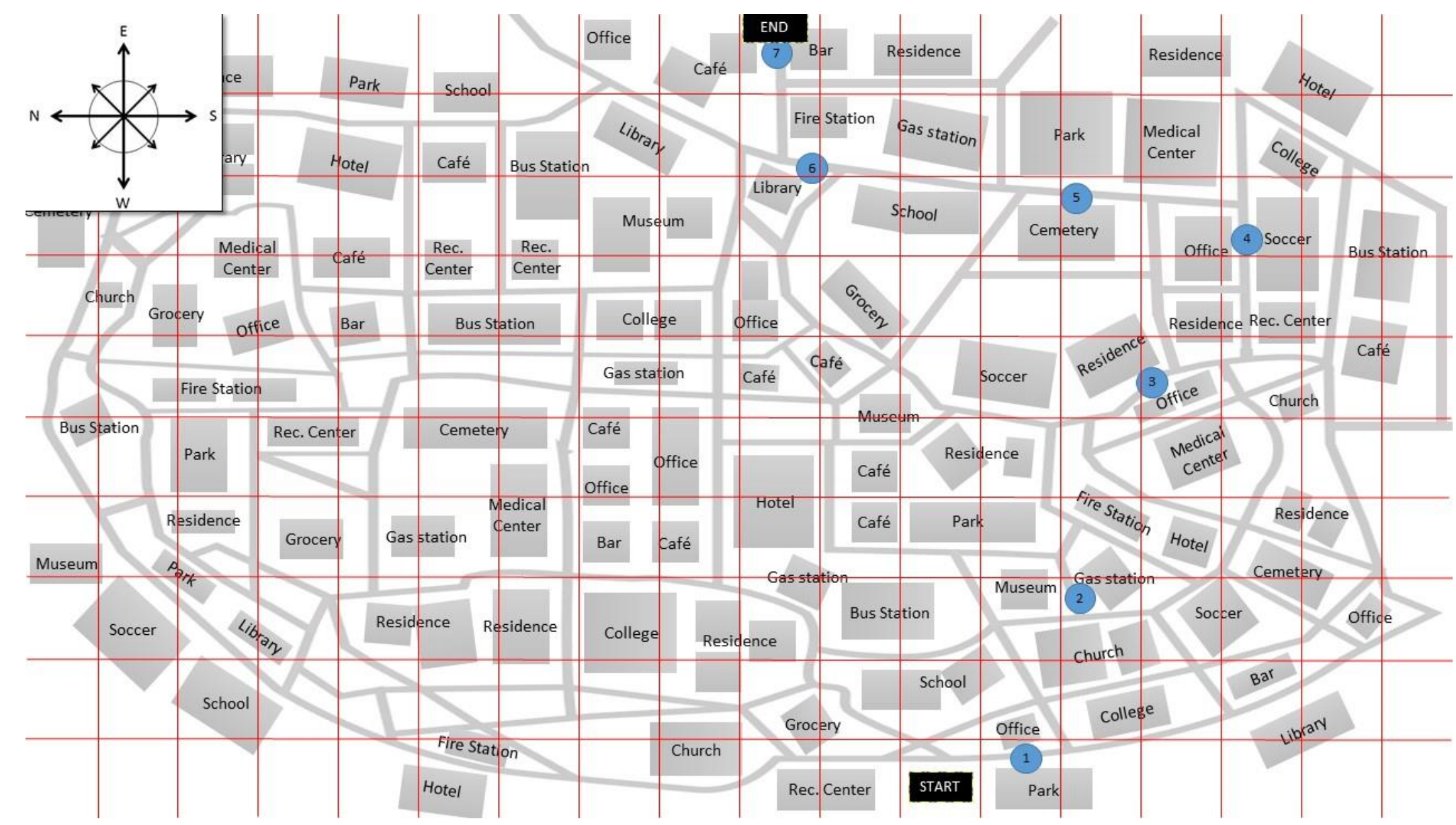

Waypoint status updates (7 updates) condition 


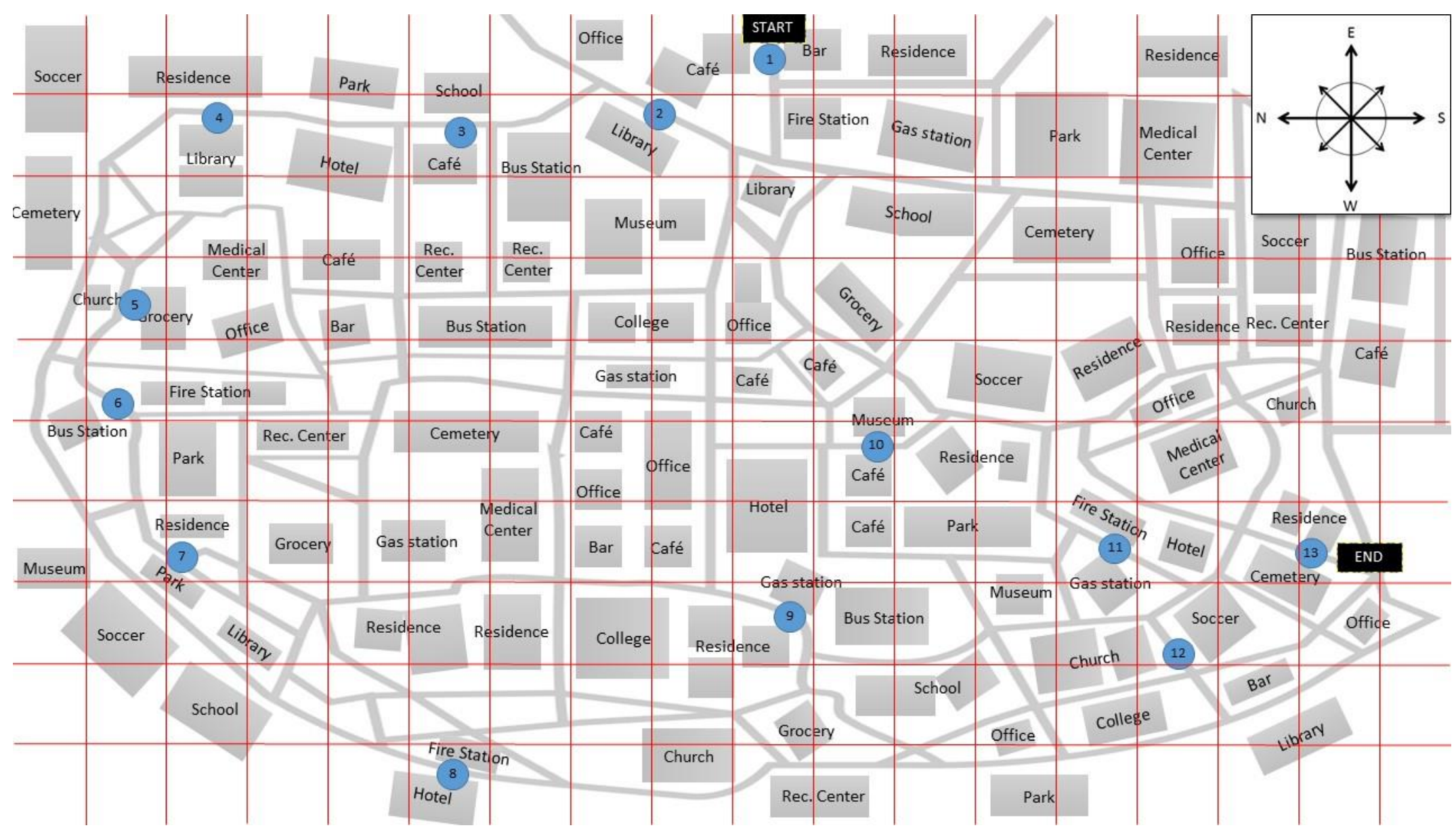

Waypoint status updates (13 updates) condition 


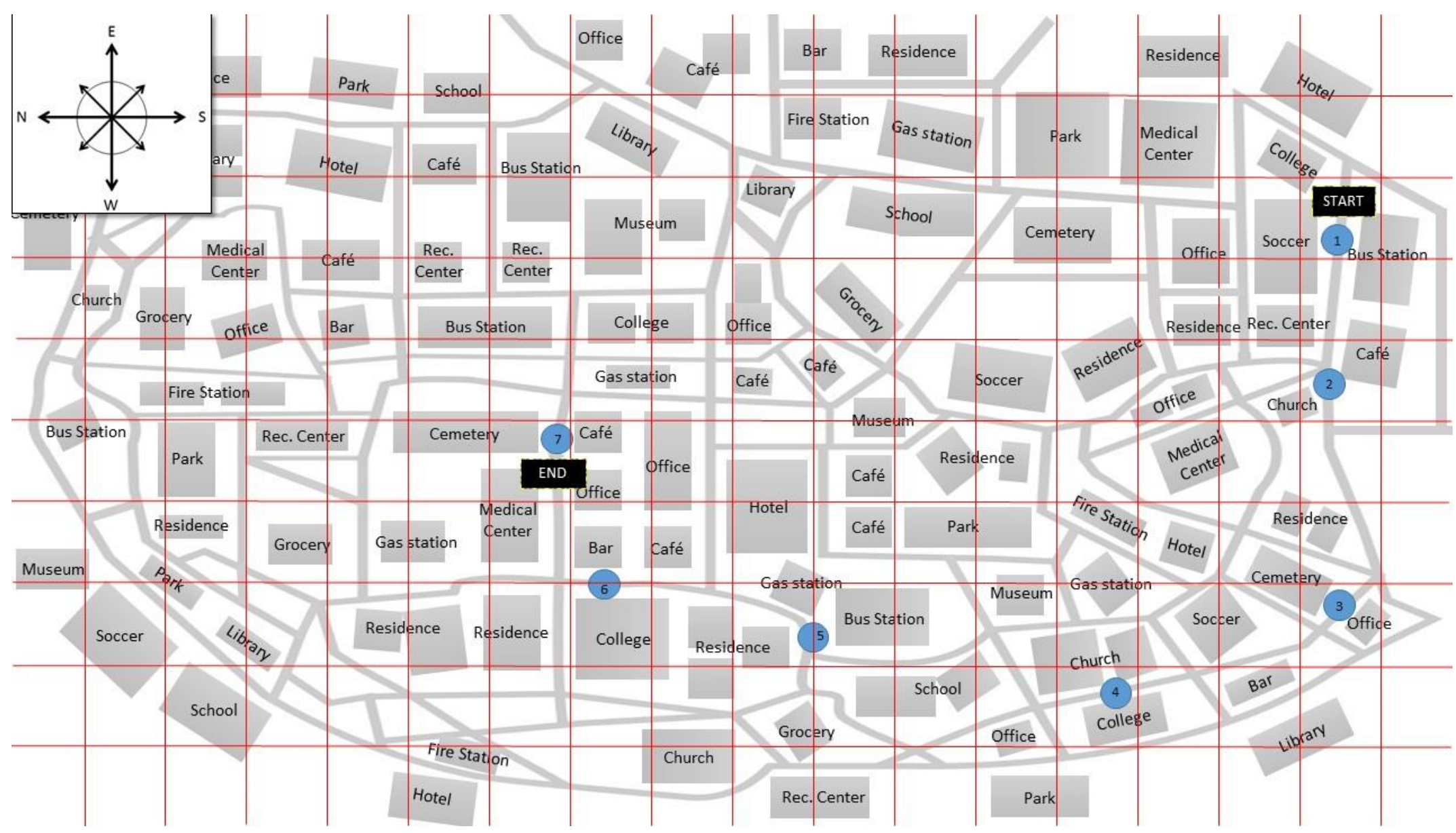

Traveling + waypoint status updates conditions with Level 1 VPT condition 


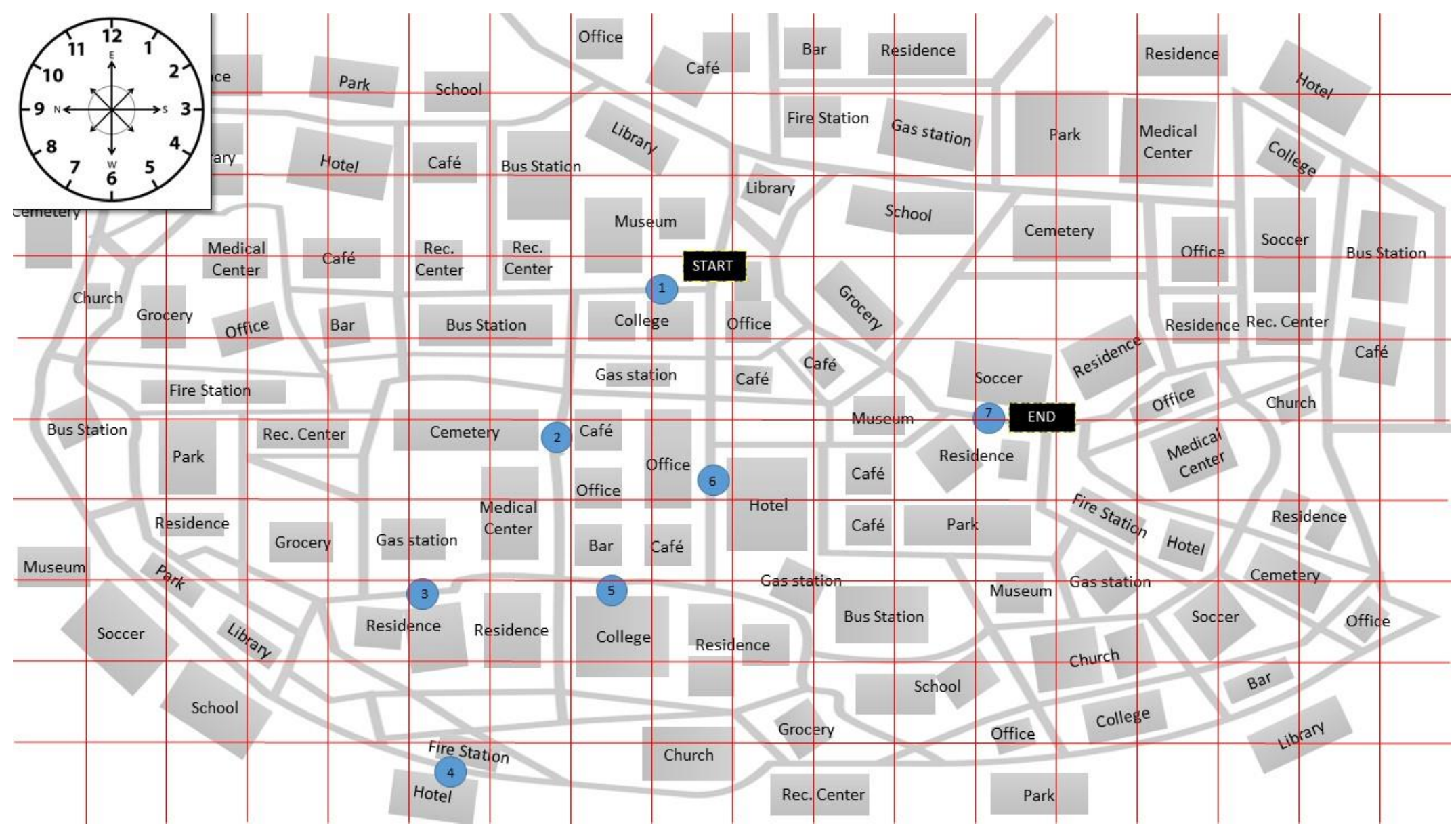

Traveling + waypoint status updates conditions with Level 2 VPT condition 


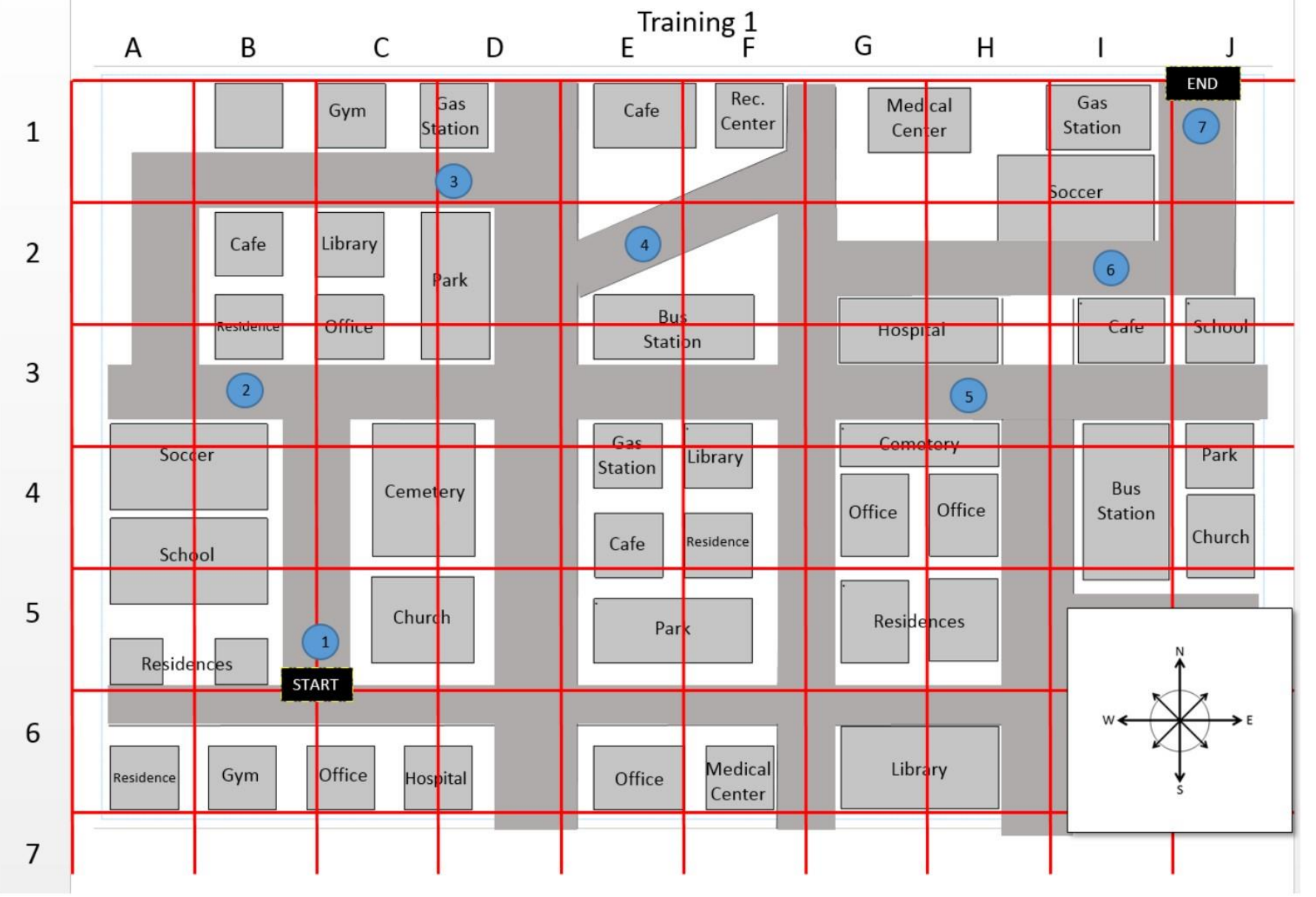




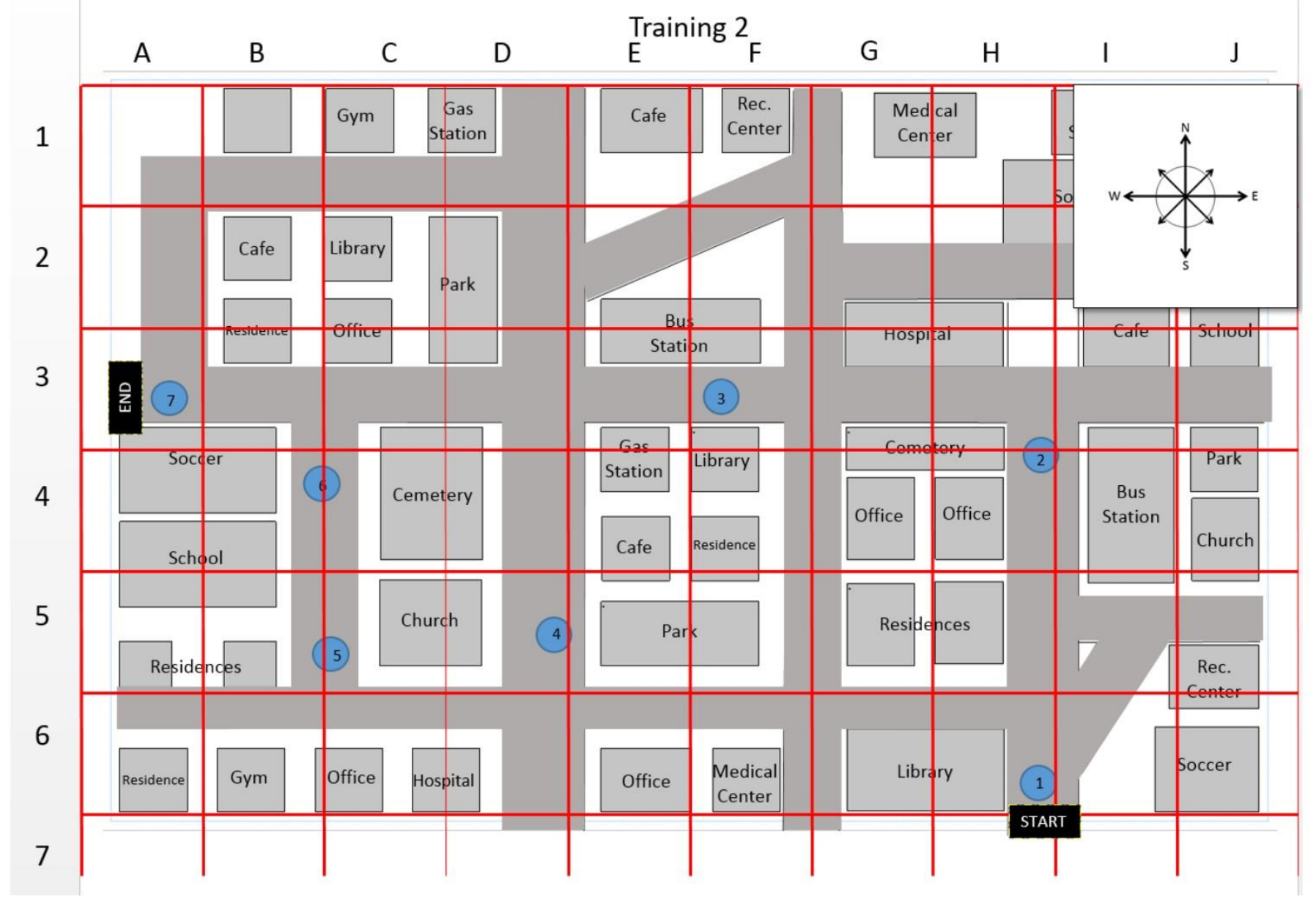




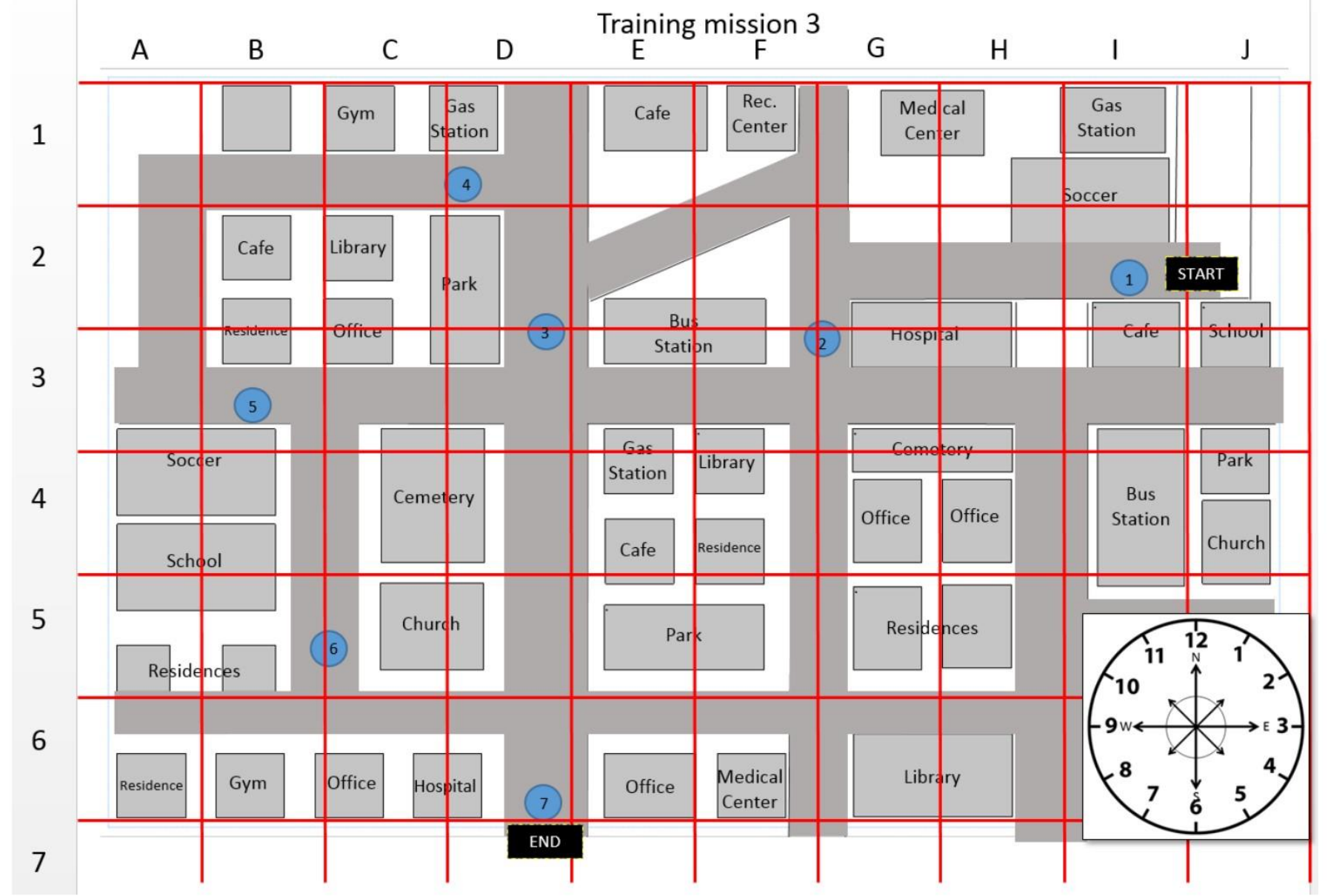




\section{APPENDIX F: REROUTING MAPS PROVIDED TO}

PARTICIPANTS 
The robot is currently located between a Soccer pitch and a Café.Please draw a route to planned waypoint 6 .

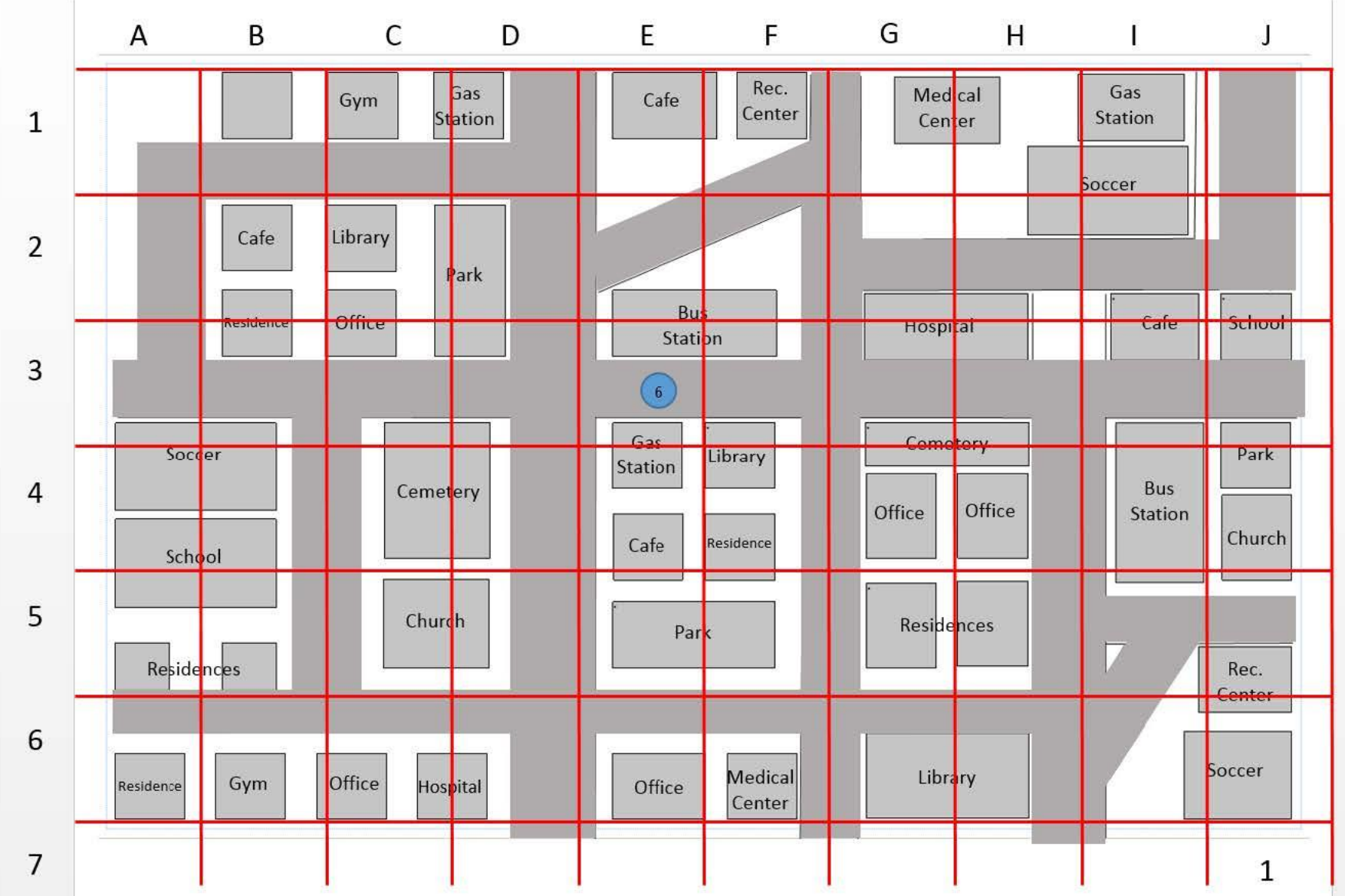




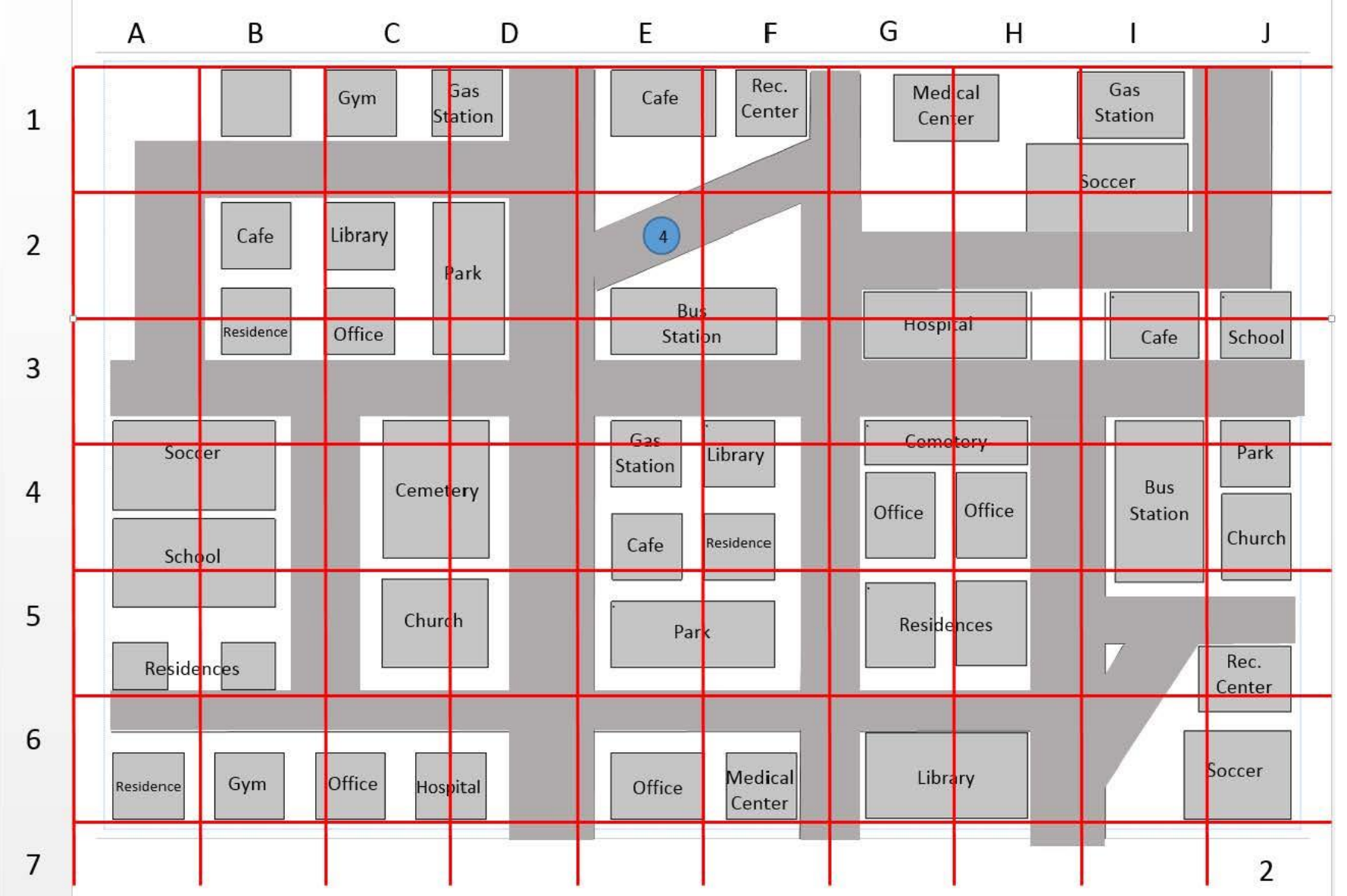




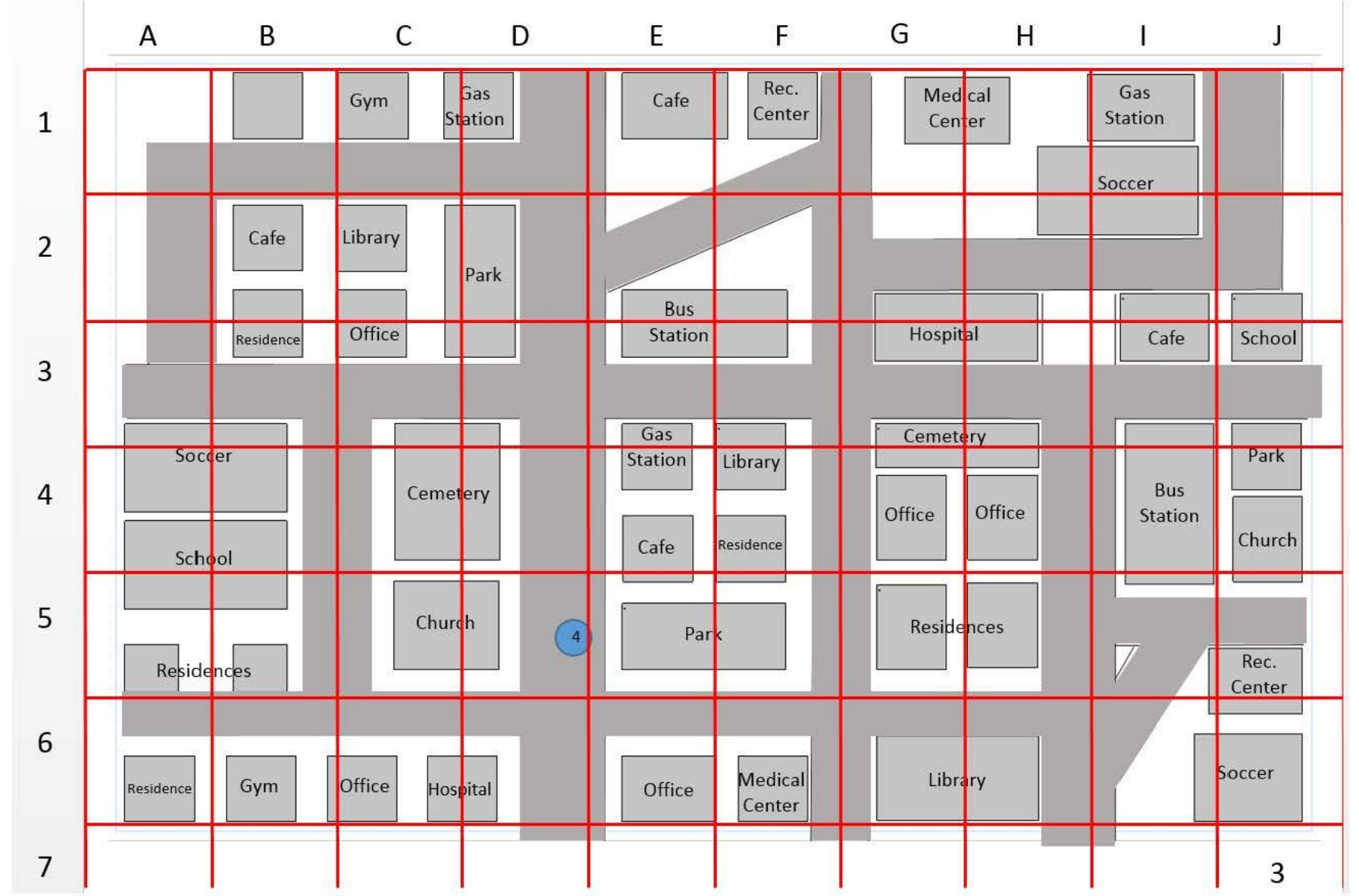




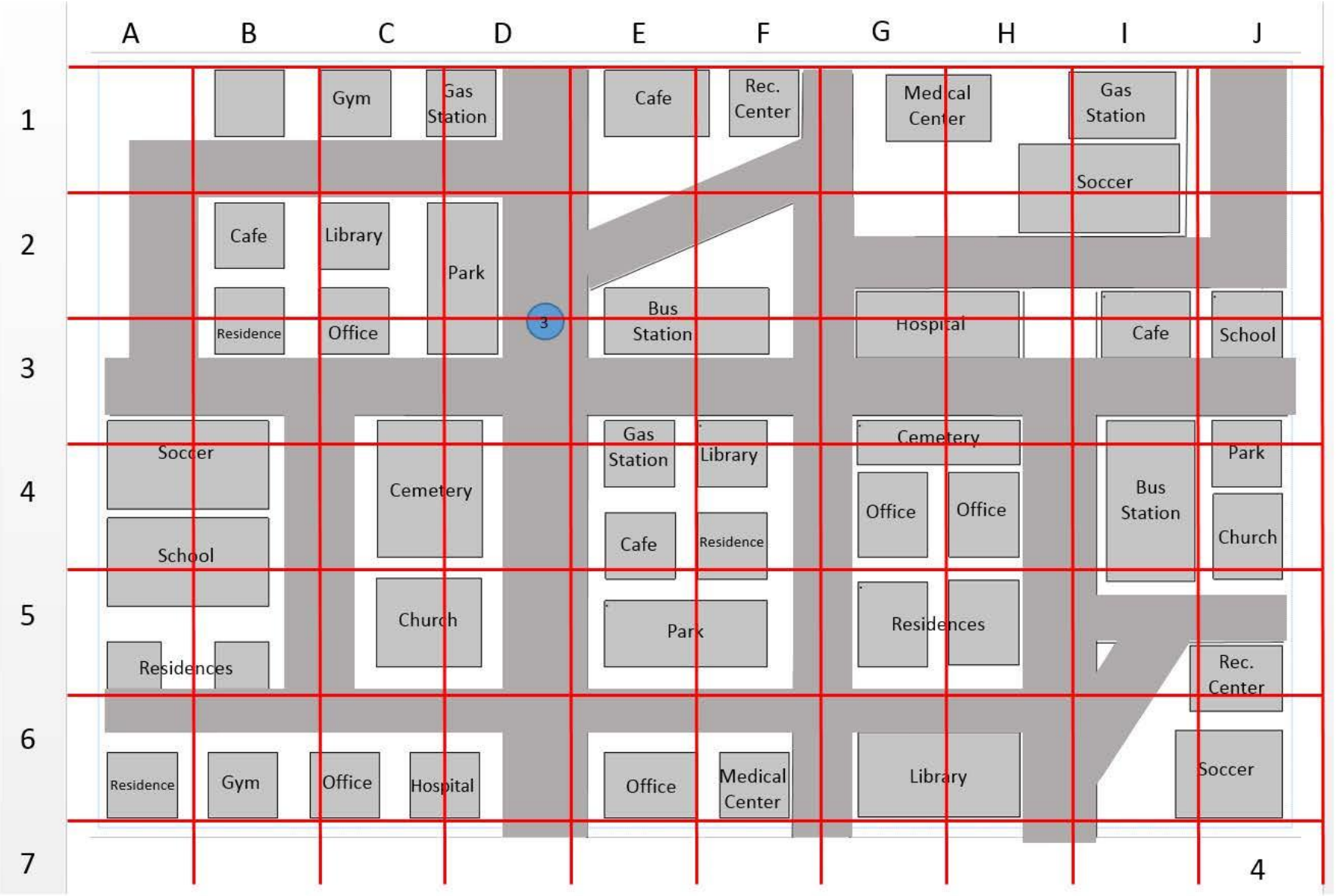




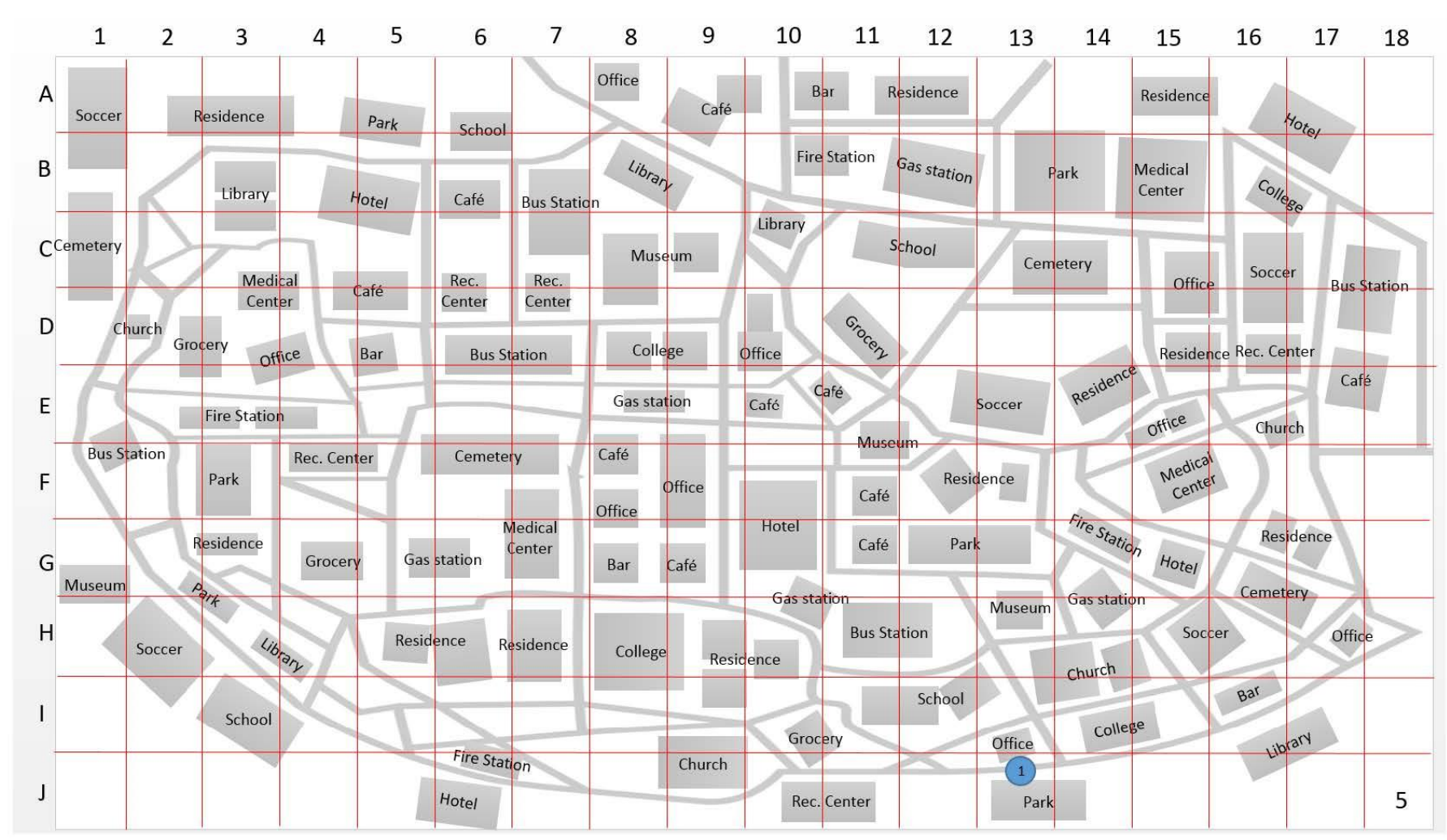




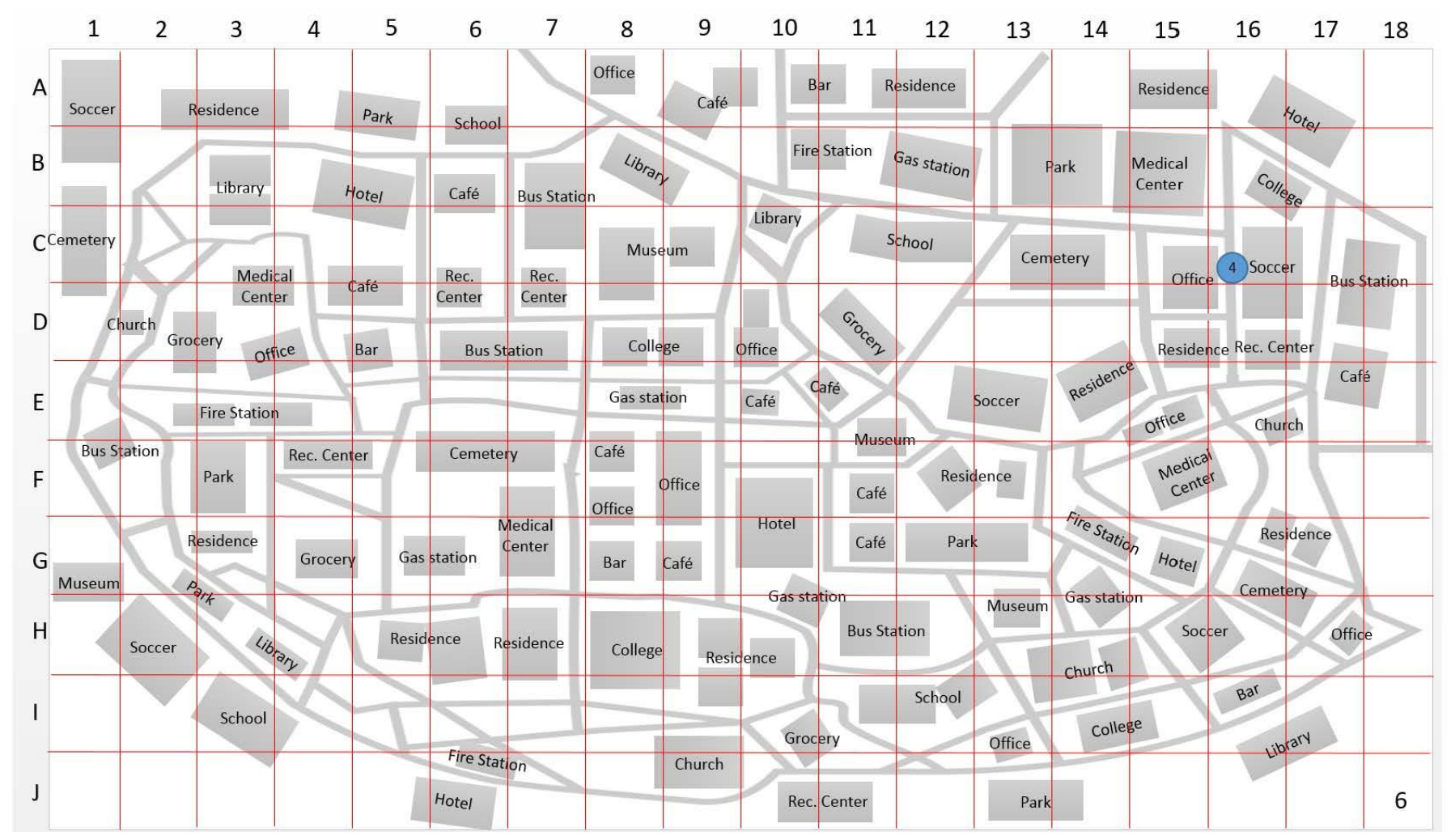




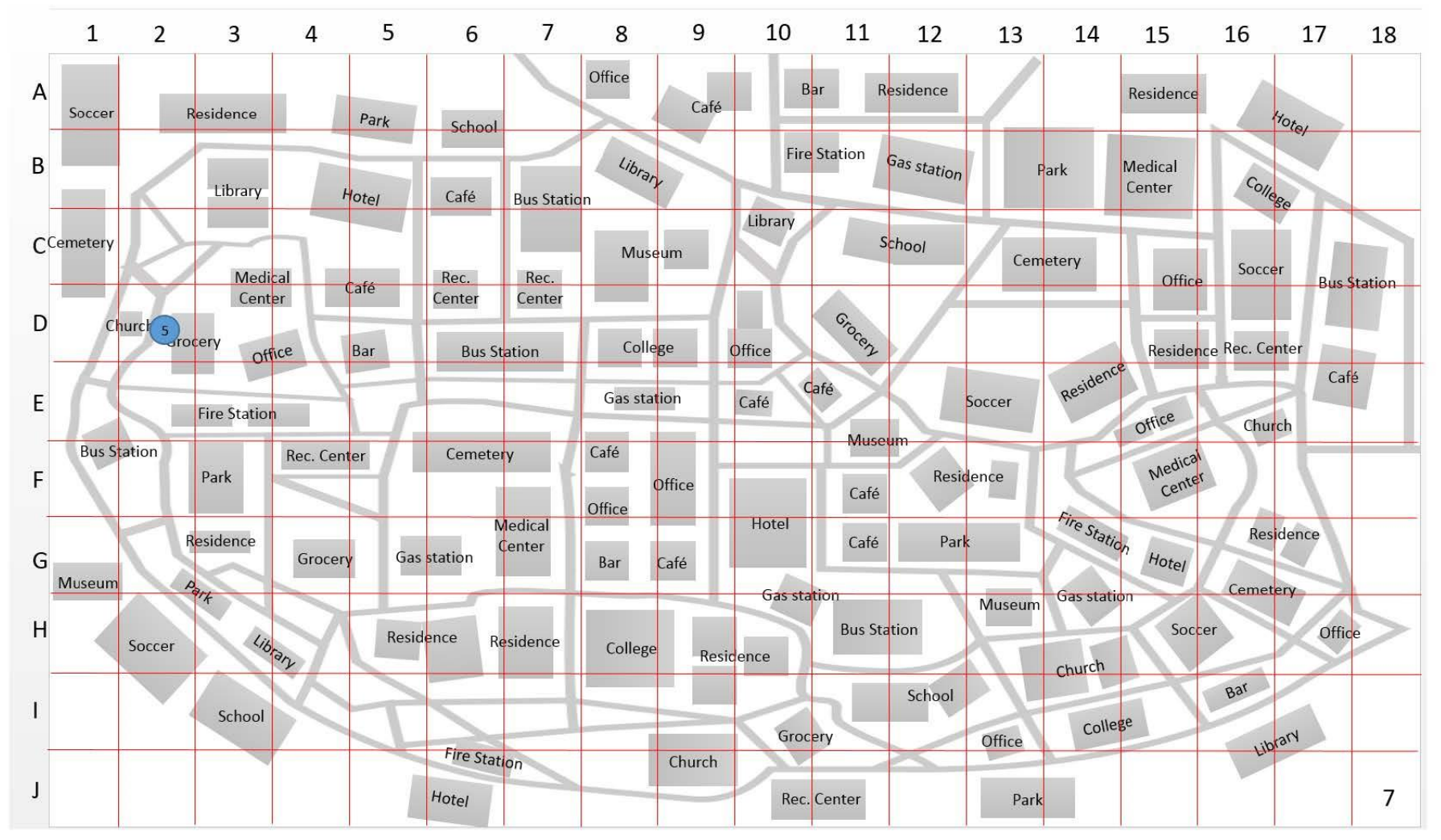




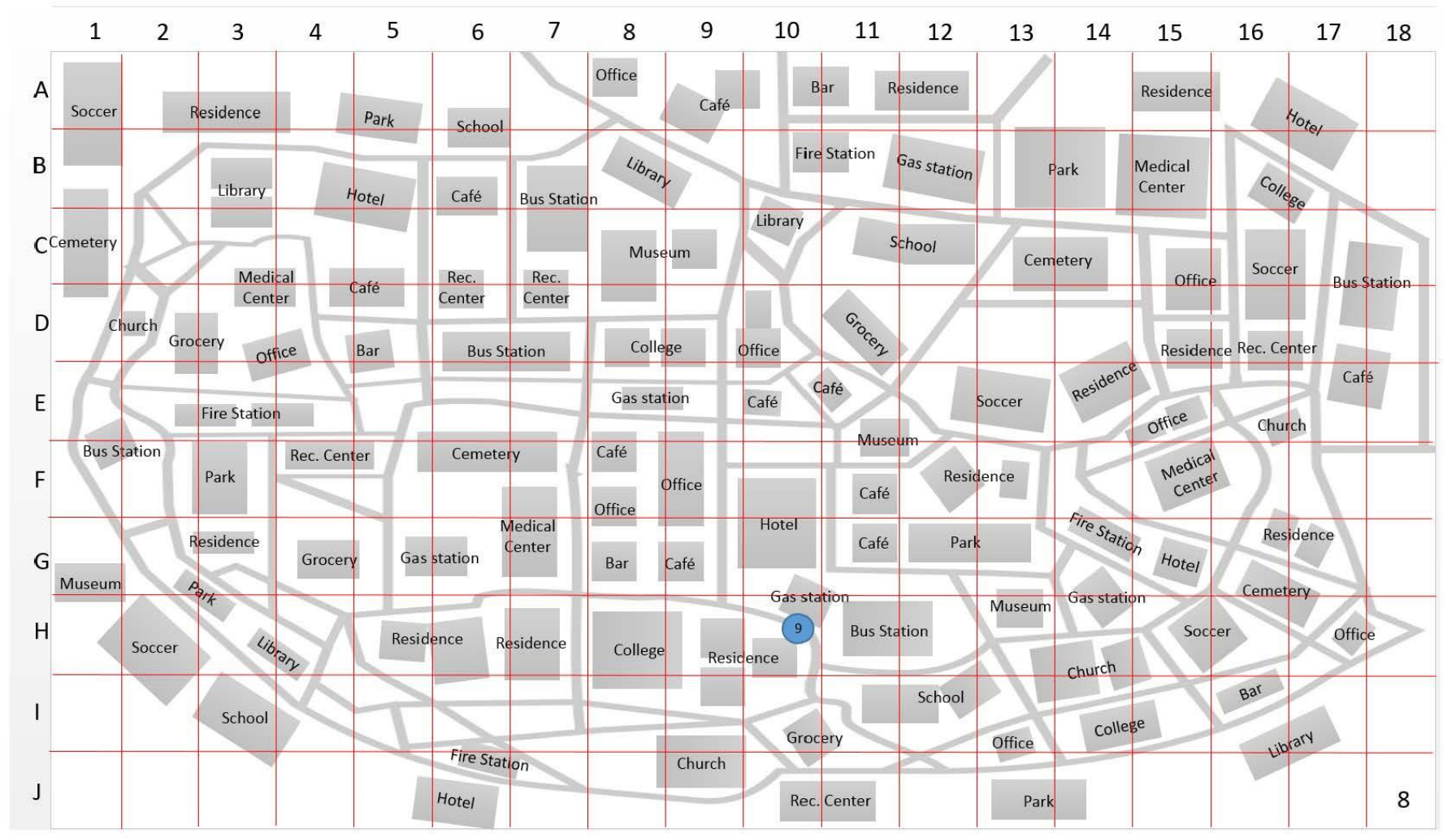




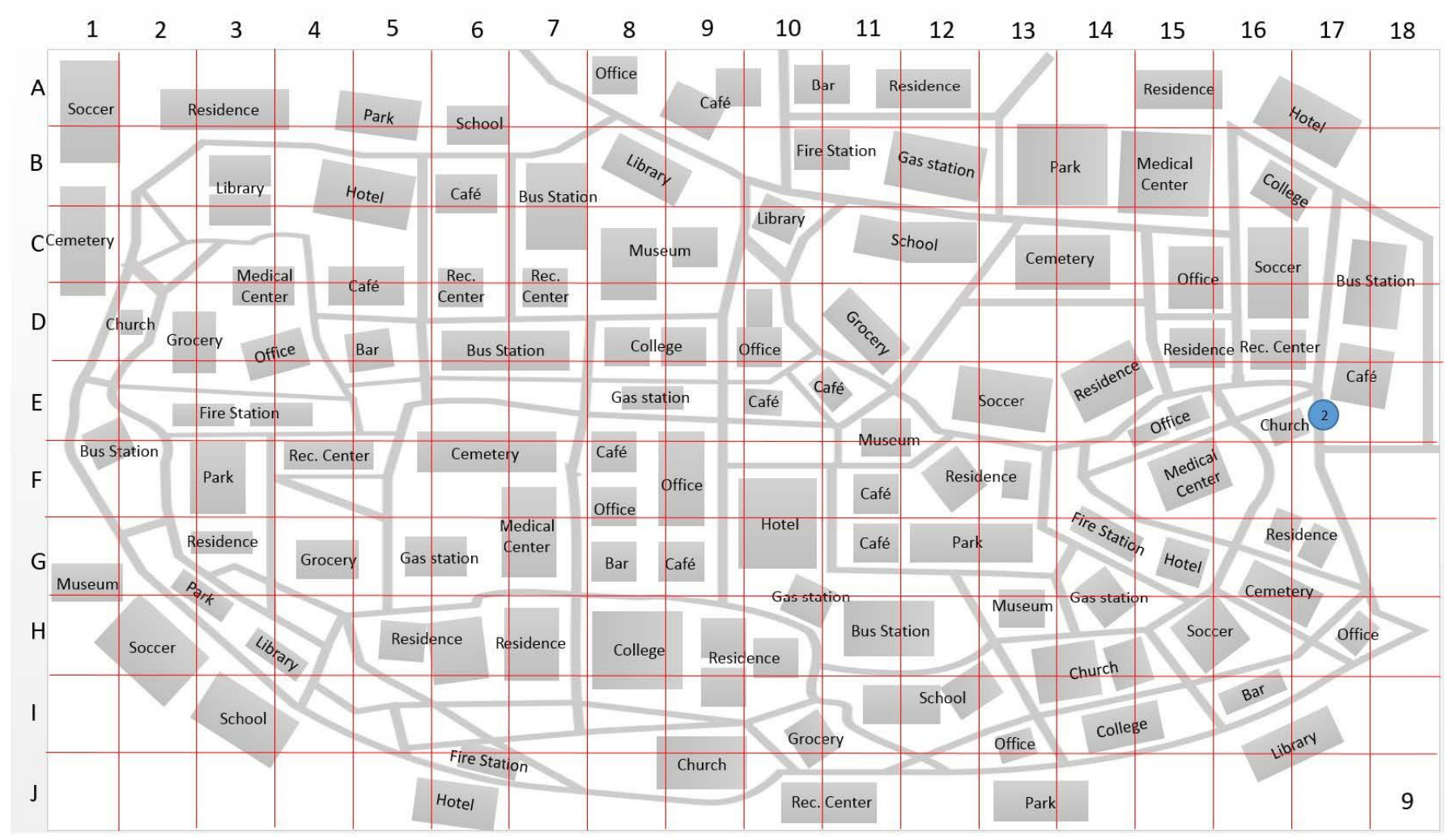




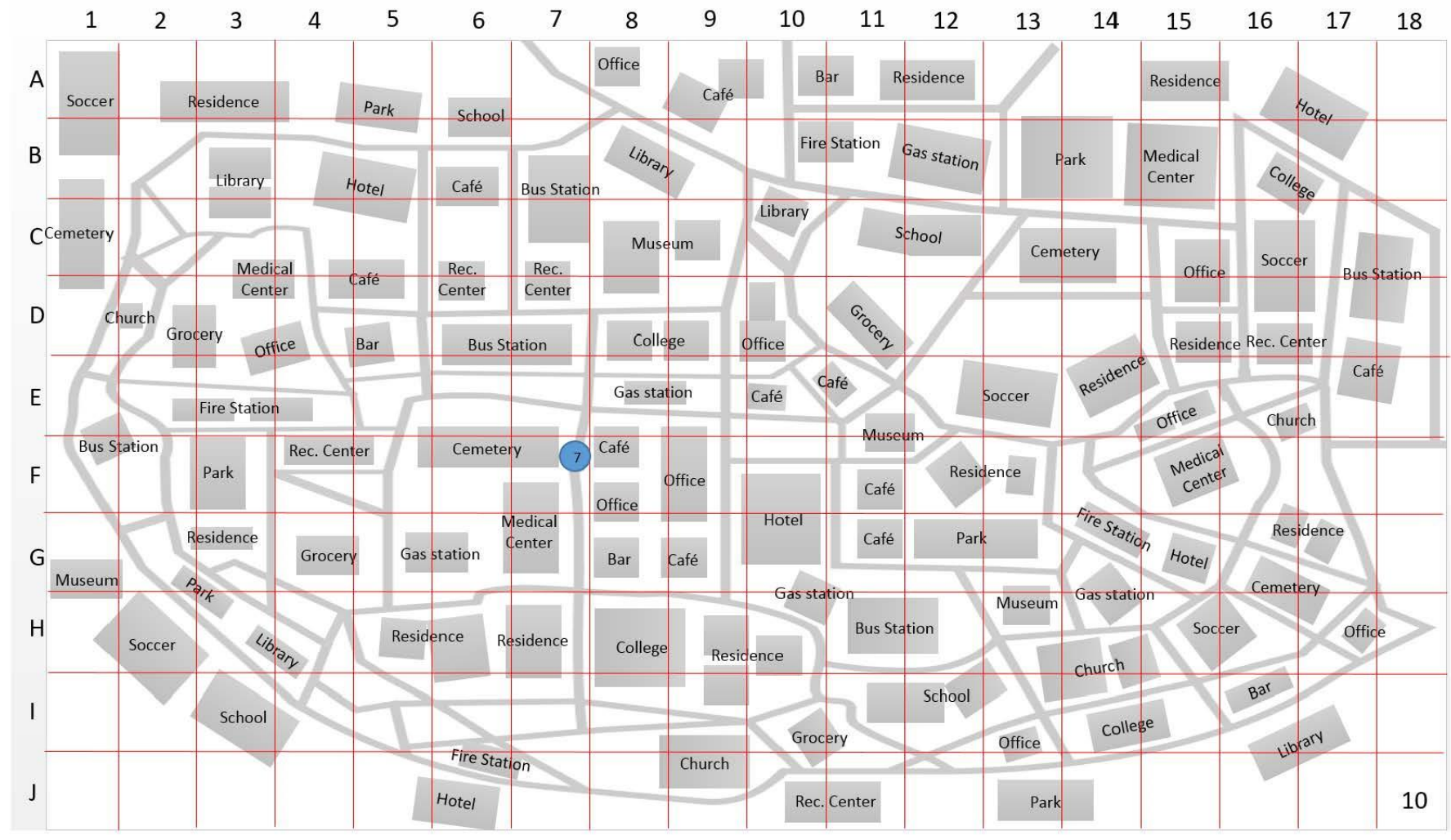




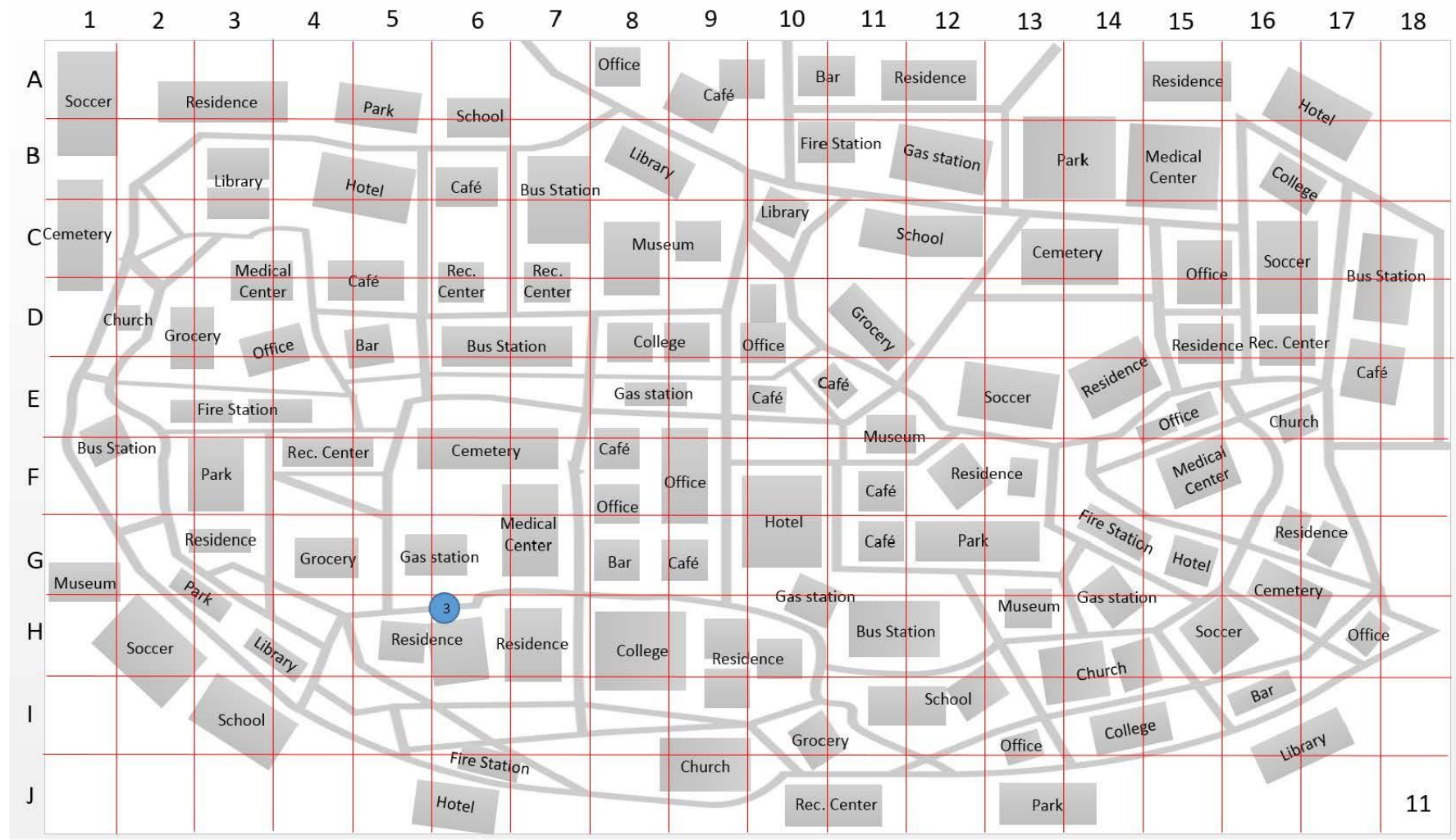




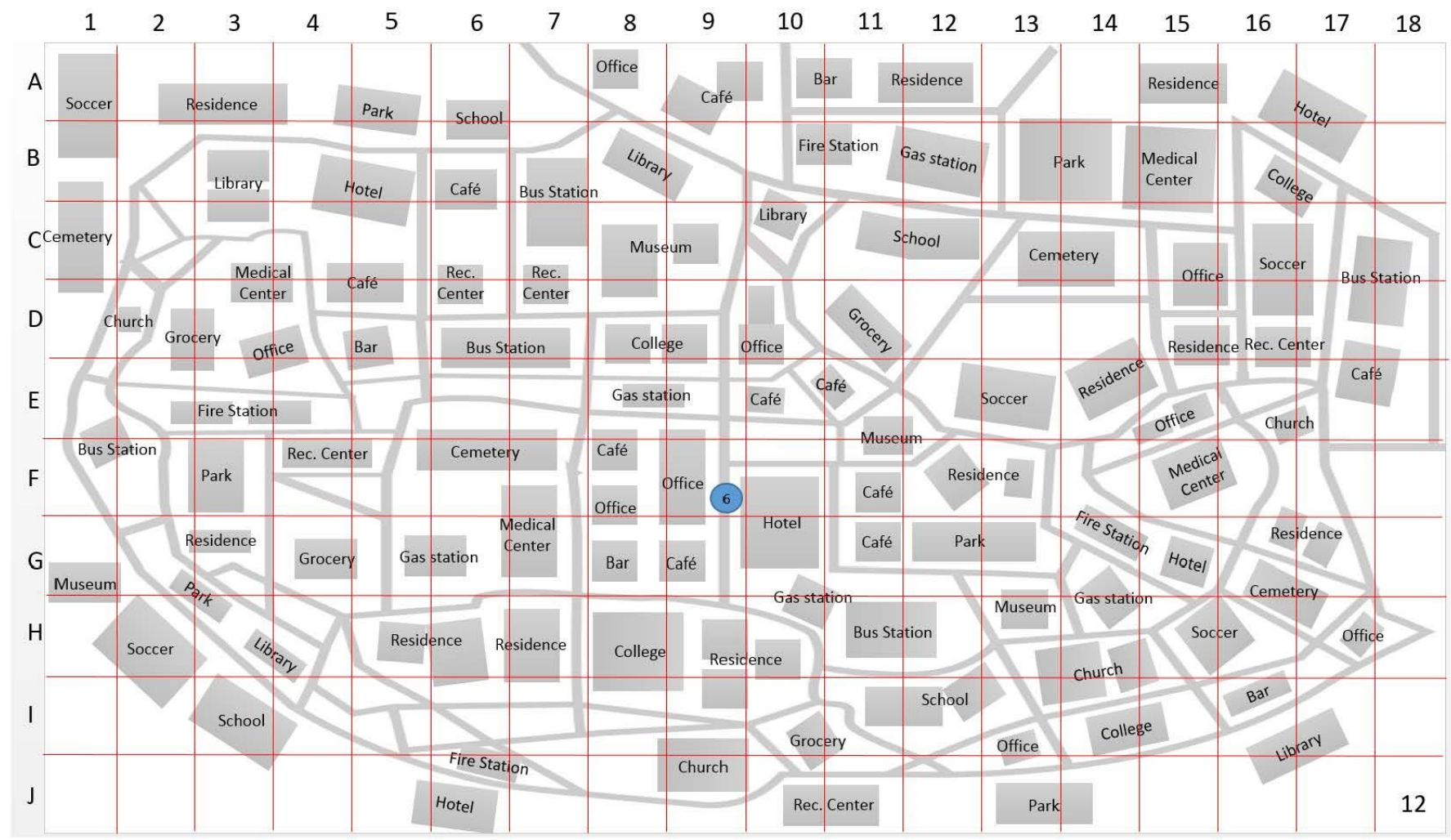


APPENDIX G: ROBOT-TO-HUMAN INFORMATION EXCHANGES FOR EXPERIMENTAL MISSION SCENARIOS 


\section{Waypoint 1}

Status: Stopped/Scanning

Time to target: $\mathbf{4 m}$ 30s Percent complete: $\mathbf{1 5 \%}$

Intel: There is a park on one side and an office on the other. 


\section{Waypoint 2}

Status: Stopped/Scanning

Time to target: $3 \mathrm{~m}$ 00s Percent complete: $\mathbf{4 0 \%}$

Intel: There is a café on one side and a hotel on the other.

\section{Waypoint 3}

Status: Stopped/Scanning

Time to target: $\mathbf{3 m} \mathbf{1 5 s}$ Percent complete: $\mathbf{2 5 \%}$

Intel: There is a residence on one side and a cemetery on the other. 


\section{Waypoint 4}

Status: Stopped/Scanning

Time to target: $\mathbf{2 m} 30 \mathrm{~s}$ Percent complete: $\mathbf{5 0 \%}$

Intel: There is a church on one side and a café on the other.

\section{Waypoint 5}

Status: Stopped/Scanning

Time to target: 1 m 30s Percent complete: $\mathbf{7 5 \%}$

Intel: There is a park on one side and a cemetery on the other. 


\section{Waypoint 6}

Status: Stopped/Scanning

Time to target: $\mathbf{1 m} \mathbf{2 0 s}$ Percent complete: $\mathbf{7 0 \%}$

Intel: There is a grocery on one side and a café on the other.

\section{Waypoint 7}

Status: Stopped/Scanning

Time to target: $\mathbf{O m}$ Os Percent complete: $\mathbf{1 0 0} \%$

Intel: There is a bar on one side and a café on the other. 


\section{Waypoint 1}

Status: Stopped/Scanning

Time to target: $\mathbf{4 m} \mathbf{3 0 s}$ Percent complete: $\mathbf{8 \%}$

Intel: There is a café on one side and an bar on the other. 


\section{Waypoint 2}

Status: Stopped/Scanning

Time to target: $\mathbf{4 m} \mathbf{4 5 s}$

Percent complete: $\mathbf{6 \%}$

Intel: There is a museum on one side and a library on the other.

\section{Waypoint 3}

Status: Stopped/Scanning

Time to target: $4 \mathrm{~m}$ 00s Percent complete: $\mathbf{2 0 \%}$

Intel: There is a bus station on one side and a college on the other. 


\section{Waypoint 4}

Status: Stopped/Scanning

Time to target: $\mathbf{3 m}$ 30s Percent complete: $\mathbf{3 0 \%}$

Intel: There is a café on one side and a hotel on the other.

\section{Waypoint 5}

Status: Stopped/Scanning

Time to target: $3 \mathrm{~m}$ 00s Percent complete: $\mathbf{4 0 \%}$

Intel: There is a church on one side and a grocery on the other. 


\section{Waypoint 6}

Status: Stopped/Scanning

Time to target: $\mathbf{2 m} \mathbf{4 0 s}$ Percent complete: $\mathbf{4 8 \%}$

Intel: There is a bus station on one side and a fire station on the other.

\section{Waypoint 7}

Status: Stopped/Scanning

Time to target: $\mathbf{2 m} \mathbf{1 5 s}$ Percent complete: $\mathbf{5 6 \%}$

Intel: There is a residence on one side and a park on the other. 


\section{Waypoint 8}

Status: Stopped/Scanning

Time to target: $\mathbf{1 m} \mathbf{5 0 s}$ Percent complete: $\mathbf{6 4 \%}$

Intel: There is a hotel on one side and a fire station on the other.

\section{Waypoint 9}

Status: Stopped/Scanning

Time to target: $1 \mathrm{~m}$ 45s Percent complete: $\mathbf{6 8 \%}$

Intel: There is a college on one side and a residence on the other. 


\section{Waypoint 10}

Status: Stopped/Scanning

Time to target: $0 \mathrm{~m}$ 50s Percent complete: $\mathbf{7 0 \%}$

Intel: There is a church on one side and a school on the other.

Waypoint 11

Status: Stopped/Scanning

Time to target: $1 \mathbf{m}$ 00s Percent complete: $\mathbf{8 2} \%$

Intel: There is a residence on one side and a soccer pitch on the other. 
Waypoint 12

Status: Stopped/Scanning

Time to target: $\mathbf{1 m} \mathbf{1 0 s}$

Percent complete: $\mathbf{7 5 \%}$

Intel: There is a office on one side and a residence on the other.

Waypoint 13

Status: Stopped/Scanning

Time to target: $1 \mathbf{m}$ 00s Percent complete: $\mathbf{9 0 \%}$

Intel: There is a church on one side and a café on the other. 


\section{Waypoint 1}

Status: Stopped/Scanning

Time to target: $\mathbf{4 m}$ 30s Percent complete: $\mathbf{1 5 \%}$

\section{Intel: There is a soccer pitch on one side and a bus station on the other.}


Status: Maneuvering

Heading: North

Towards next waypoint

\section{Waypoint 2}

Status: Stopped/Scanning

Time to target: $\mathbf{4 m}$ Os

Percent complete: $\mathbf{3 5 \%}$

Intel: There is a residence on one side and a office on the other. 
Status: Maneuvering

Heading: Northwest

Towards next waypoint

\section{Waypoint 3}

Status: Stopped/Scanning

Time to target: $\mathbf{2 m} \mathbf{4 5 s}$ Percent complete: $\mathbf{5 0 \%}$

Intel: There is a park on one side and a museum on the other. 
Status: Maneuvering

Heading: West-southwest

Towards next waypoint

\section{Waypoint 4}

Status: Stopped/Scanning

Time to target: $\mathbf{2 m} \mathbf{1 5 s}$ Percent complete: $\mathbf{6 0 \%}$

Intel: There is a church on one side and a college on the other. 
Status: Maneuvering

Heading: North-northeast

Towards next waypoint

\section{Waypoint 5}

Status: Stopped/Scanning

Time to target: $\mathbf{1 m} \mathbf{4 5 s}$ Percent complete: $\mathbf{7 0 \%}$

Intel: There is a grocery on one side and a school on the other. 
Status: Maneuvering

Heading: East

Towards next waypoint

\section{Waypoint 6}

Status: Stopped/Scanning

Time to target: $\mathbf{0 m} \mathbf{4 5 s}$ Percent complete: $\mathbf{9 0 \%}$

Intel: There is an office on one side and a college on the other. 
Status: Maneuvering

Heading: West

Towards next waypoint

\section{Waypoint 7}

Status: Stopped/Scanning

Time to target: $\mathbf{0 m}$ Os Percent complete: $\mathbf{1 0 0} \%$

Intel: There is a cemetery on one side and a café on the other. 
Experimental mission 4: Traveling + waypoint status updates with Level 2 VPT

condition

Waypoint 1

Status: Stopped/Scanning

Time to target: $\mathbf{4 m}$ 30s

Percent complete: $\mathbf{1 5 \%}$

Intel: There is a museum on one side and a college on the other. 
Status: Maneuvering

Heading: 6 o'clock, West

Towards next waypoint

\section{Waypoint 2}

Status: Stopped/Scanning

Time to target: $\mathbf{3 m} \mathbf{4 5 s}$

Percent complete: $\mathbf{4 0 \%}$

Intel: There is a grocery on one side and a gas station on the other. 
Status: Maneuvering

Heading: 7 o'clock, West-northwest

Towards next waypoint

\section{Waypoint 3}

Status: Stopped/Scanning

Time to target: $3 \mathrm{~m} \mathrm{00s}$ Percent complete: $\mathbf{4 5 \%}$

Intel: There is a school on one side and a library on the other. 
Status: Maneuvering

Heading: 4 o'clock, Southsouthwest

Towards next waypoint

\section{Waypoint 4}

Status: Stopped/Scanning

Time to target: $\mathbf{2 m} \mathbf{1 5 s}$ Percent complete: $\mathbf{6 0 \%}$

Intel: There is a hotel on one side and a fire station on the other. 
Status: Maneuvering

Heading: 2 o'clock, Southsoutheast

Towards next waypoint

\section{Waypoint 5}

Status: Stopped/Scanning

Time to target: $\mathbf{2 m} 30 \mathrm{~s}$ Percent complete: $\mathbf{5 5 \%}$

Intel: There is a grocery on one side and a residence on the other. 
Status: Maneuvering

Heading: 3 o'clock, South

Towards next waypoint

\section{Waypoint 6}

Status: Stopped/Scanning

Time to target: $\mathbf{2 m} \mathbf{4 5 s}$ Percent complete: $\mathbf{6 5 \%}$

Intel: There is an church on one side and a museum on the other. 
Status: Maneuvering

Heading: 9 o'clock, North

Towards next waypoint

\section{Waypoint 7}

Status: Stopped/Scanning

Time to target: $\mathbf{2 m}$ Os Percent complete: $\mathbf{8 0} \%$

Intel: There is a hotel on one side and a café on the other. 


\section{APPENDIX H: PLANNED ANALYSES AND EXPECTED}

PATTERNS OF RESULTS 
Planned analyses, and expected pattern of results

Several paired comparisons among individual experimental conditions were planned to analyze the results of this study.

Effects of VPT on measures of SA regarding the robot and human assistance provided to the robot

A significant difference in scores on the measure of SA regarding the robot was expected between the Traveling + waypoint updates with Level 1 VPT condition and the Traveling + waypoint updates with Level 2 VPT condition (i.e., Missions 3 and 4), such that SA scores in Mission 4 were expected to be significantly higher than in Mission 3 (see Figure 31).

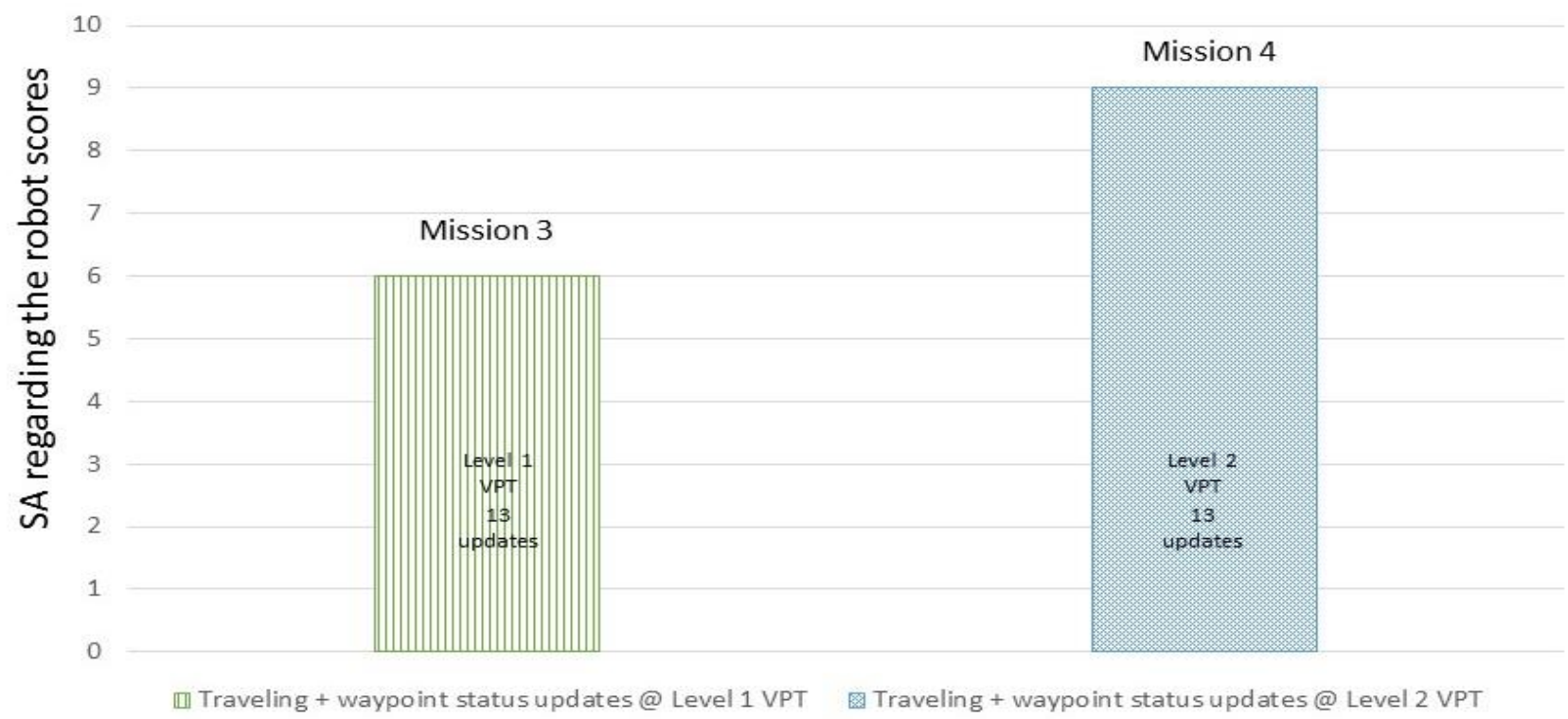

Figure 31. Contrast of scores on the measure of SA regarding the robot in Missions 3 and 4. 
This pattern of results would show the benefit of adding Level 2 VPT context over Level 1 VPT context to robot-to-human information transfers for scores on the SA regarding the robot measure, and lend support for Hypothesis H4.

In addition, a significant difference in scores on the measure of human assistance provided to the robot was expected between the Traveling + waypoint updates with Level 1 VPT condition and the Traveling + waypoint updates with Level 2 VPT condition (i.e., Missions 3 and 4). Scores on the measure of human assistance provided to the robot in Mission 4 were expected to be significantly higher than in Mission 3 (see Figure 32).

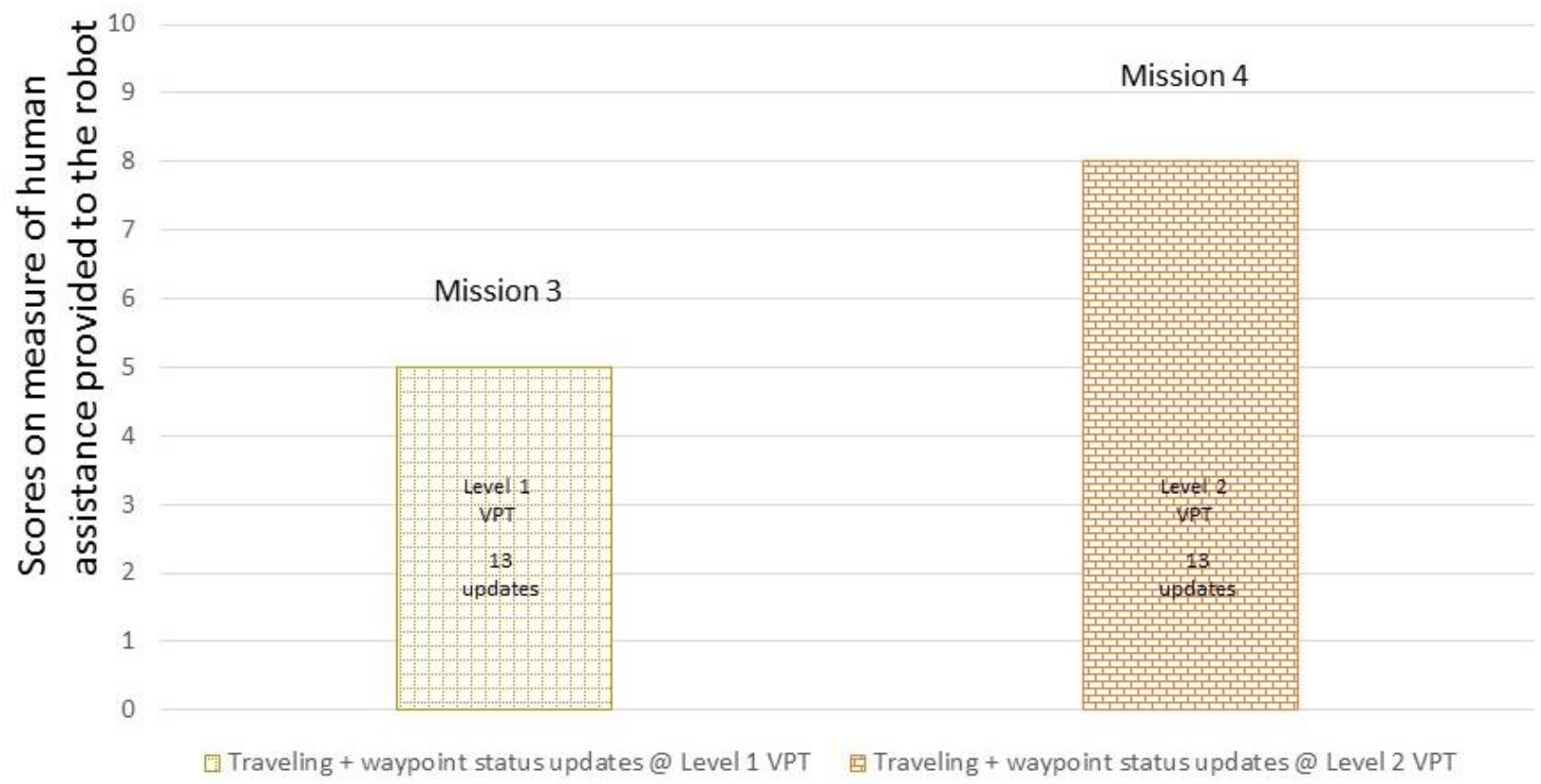

Figure 32. Contrast of scores on the measure of human assistance provided to the robot in Missions 3 and 4. 
This pattern of results would show the benefit of adding Level 2 VPT context over Level 1 VPT context to robot-to-human information exchanges, for scores on the measure of human assistance provided to the robot, and lend support for Hypothesis H5.

Effects of number of updates on measures of SA regarding the robot and human assistance provided to the robot

A significant difference in scores on the measure of SA regarding the robot was expected between the Waypoint status updates (A) condition and the Waypoint status updates (B) condition (i.e., Mission 1 and Mission 2). Significantly higher scores on the measure of SA regarding the robot were expected in Mission 2 than in Mission 1 (see Figure 33)

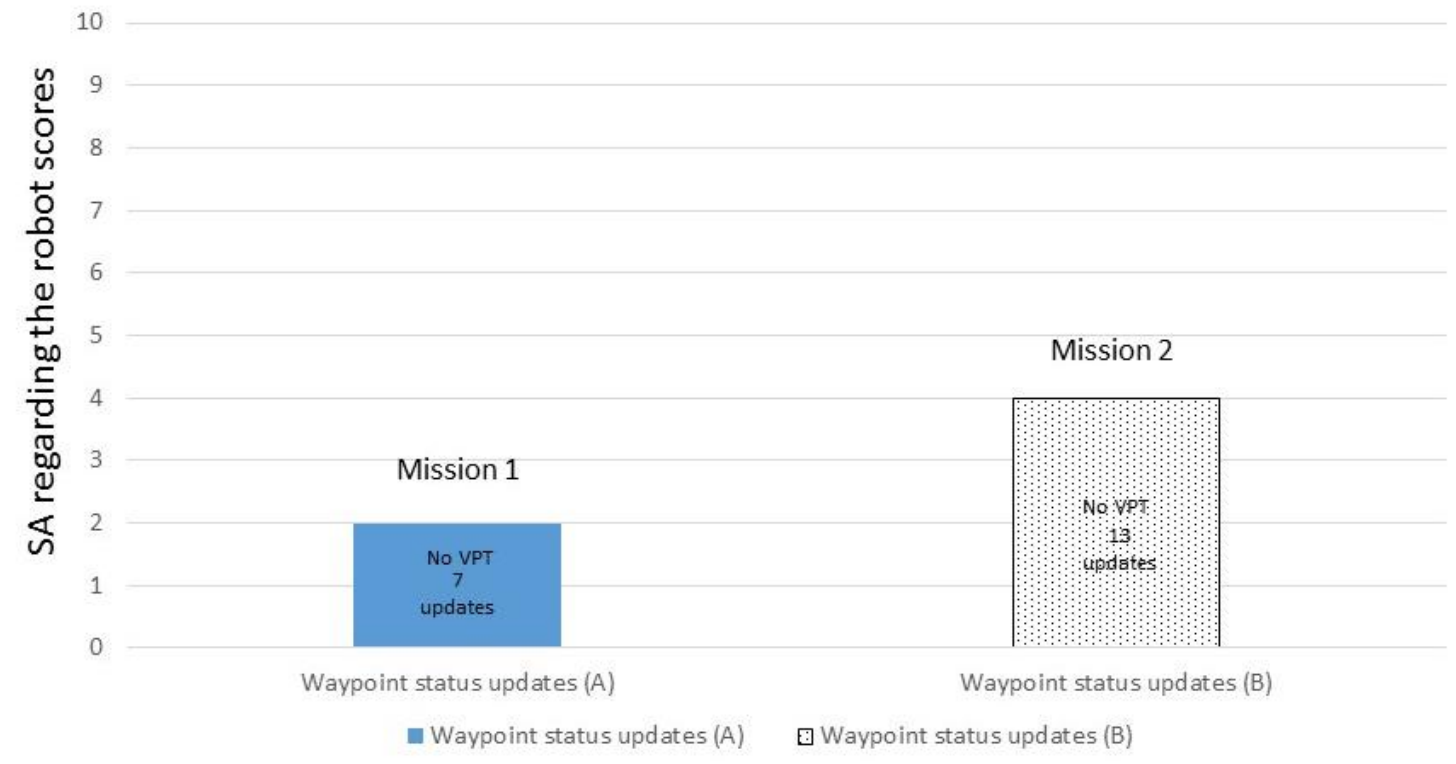

Figure 33. Contrast of scores on the measure of SA regarding the robot in Missions 1 and 2. 
This pattern of results would show a positive benefit of increasing the number of updates provided to the human by the robot on SA regarding the robot scores and lend support for Hypothesis $\mathrm{H} 2$.

In addition, a significant difference in scores on the measure of human assistance provided to the robot was expected between the Waypoint status updates (A) condition and the Waypoint status updates (B) condition (i.e., Mission 1 and Mission 2). Significantly higher scores on the measure of human assistance provided to the robot were expected in Mission 1 than in Mission 2 (see Figure 34)

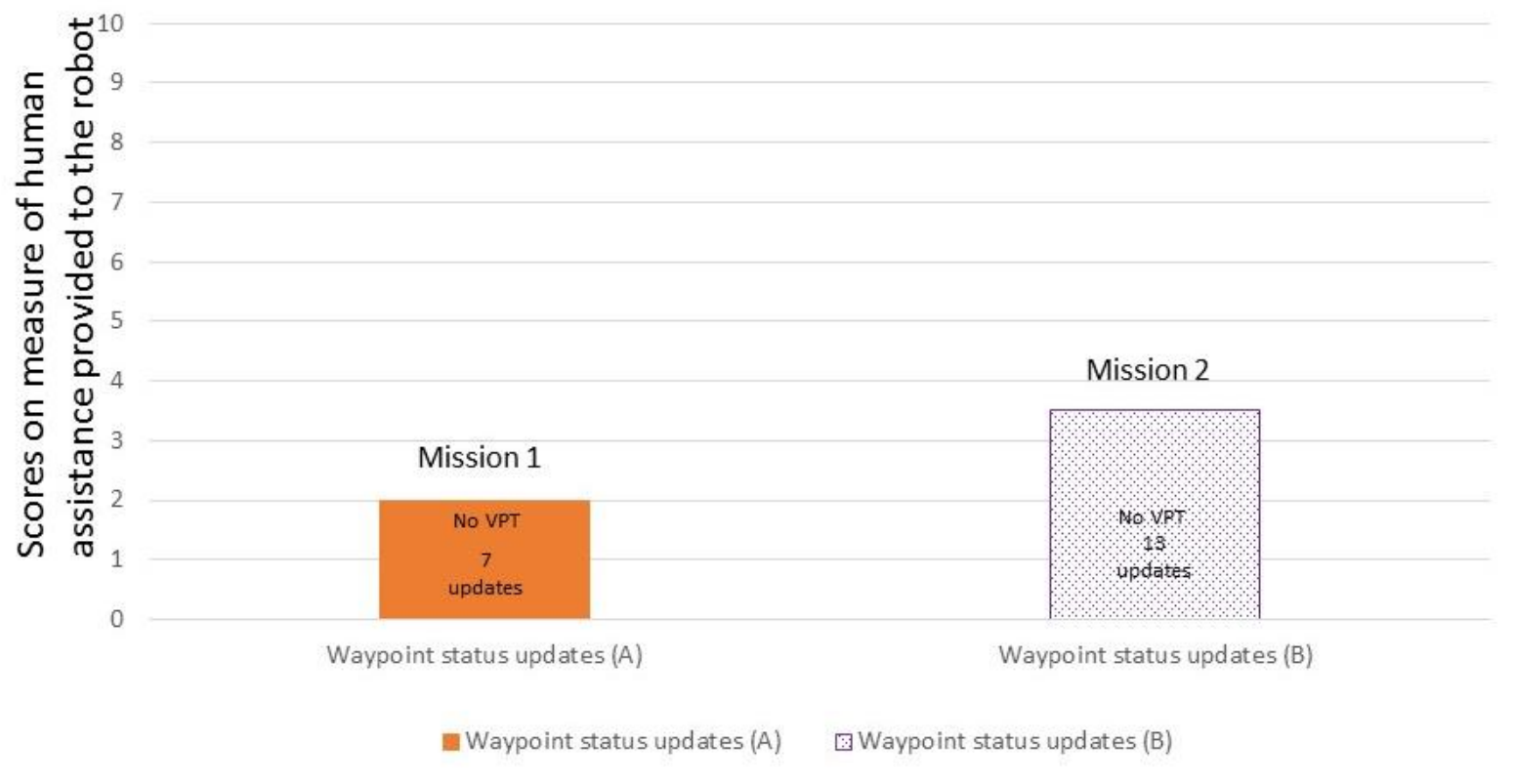

Figure 34. Contrast of scores on the measure of human assistance provided to the robot in Missions 1 and 2. 
This pattern of results would show a positive benefit of increasing the number of updates provided to the human by the robot on scores on the measure of human assistance provided to the robot and lend support for Hypothesis H3.

Additional analyses: VPT vs. number of updates on measures of SA and human assistance provided to the robot

Additional paired comparison analyses were planned to examine the benefit of adding VPT context to robot-to-human information exchanges over simply adding more robot-to-human information exchanges. Specifically, a comparison of scores between the Waypoint status updates (B) condition (i.e., Mission 2) and the Traveling + waypoint updates with Level 1 VPT condition (i.e., Mission 3) was planned to be conducted. The difference between these two missions was the addition of VPT information. In both missions, participants received equal numbers of robot-to-human information exchanges. However, in Mission 3, participants received some updates (i.e., the Traveling status updates) with VPT context added (i.e., Level 1 VPT). As such a comparison of scores on the measures SA regarding the robot, and a comparison of scores on the measure of human assistance provided to the robot between the Waypoint status updates (B) condition (i.e., Mission 2) and the Traveling + waypoint status updates with Level 1 VPT condition (i.e., Mission 3) was planned for the analyses.

Higher scores on the measure of SA regarding the robot were expected in Mission 3 than in Mission 2. In addition, higher scores on the measure of human assistance 
provided to the robot were expected in Mission 3 than in Mission 2. These patterns of results would lend support for the notion that VPT context is a more important component to building SA regarding the robot, and providing assistance to the robot than simply adding more information transfers alone (see Figures 35 and 36).

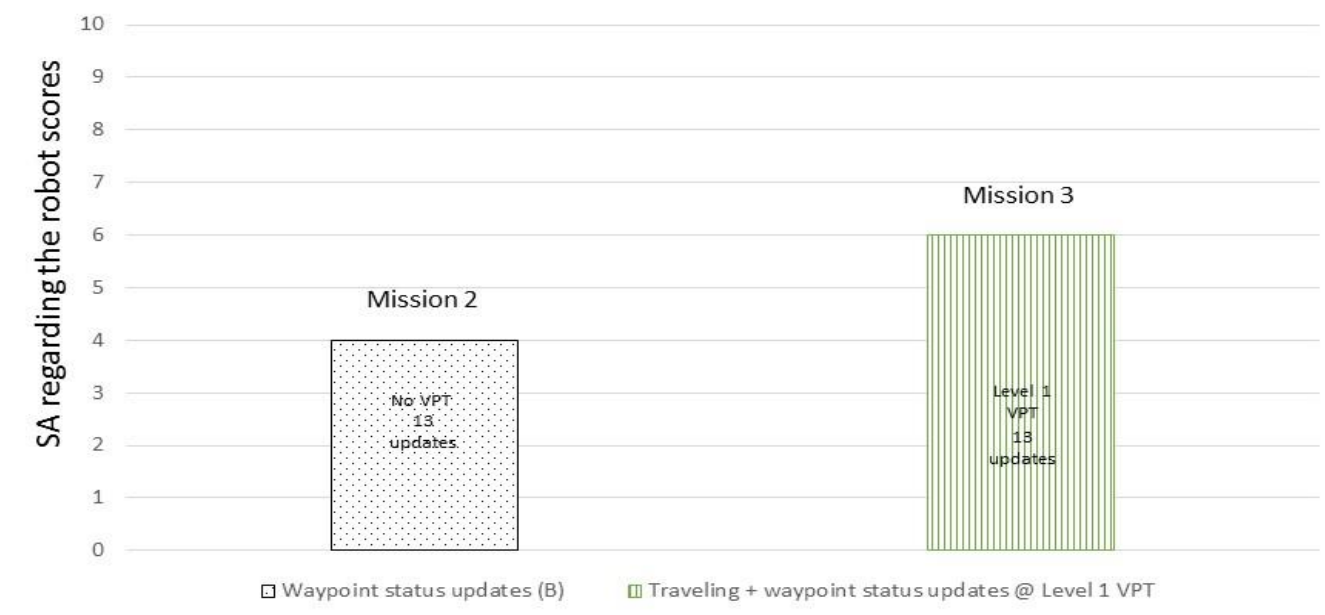

Figure 35. Contrast of scores on the measure of SA regarding the robot in Missions 2 and 3.

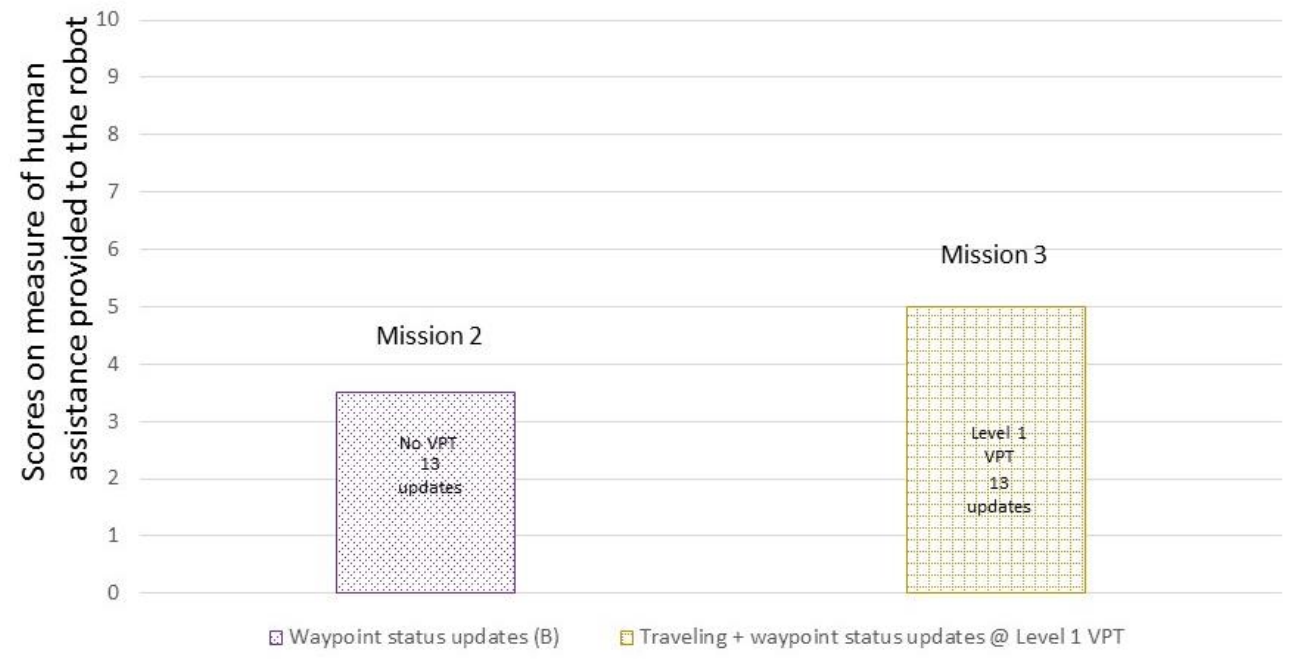

Figure 36. Contrast of scores on the measure of human assistance provided to the robot in Missions 2 and 3. 


\section{Performance on change detection task}

It should be noted that the above listed patterns of results would support the ideas that increasing the number of robot-to-human information exchanges and adding higher Levels of VPT context to those exchanges, would improve scores on the measures of SA regarding the robot, and human assistance provided to the robot. However, it is important and practical to investigate the effects of these manipulations on dual task performance (i.e., the change detection task). The future vision of Soldier-robot teams in one in which the robot and the Soldier complete separate, but interdependent tasks. If the robot's information exchanges hinder the Soldier's ability to perform his/her individual tasks, then the practical utility of robot-to-human information exchanges will be diminished. As such, a number of paired comparisons were planned to examine the effects of the number of robot-to-human information exchanges and Level of VPT on dual task performance.

\section{Effects of number of robot-to-human information exchanges on change detection task}

\section{performance}

Differences in scores on the change detection task were expected between the Waypoint status updates (A) condition and the Waypoint status updates (B) condition (i.e., Mission 1 and Mission 2). Such that, a reduction in change detection task performance was expected as the number of updates from the robot increased (see Figure 37). 


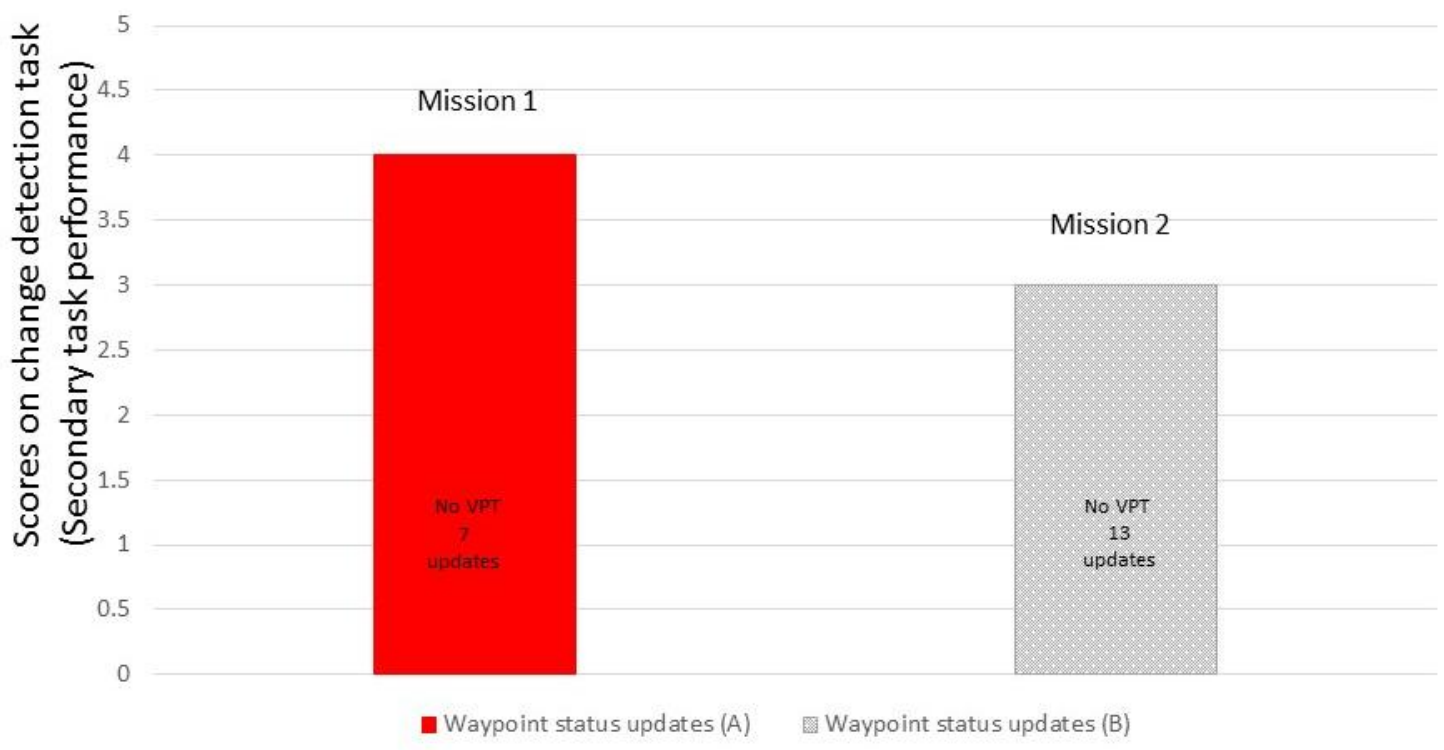

Figure 37. Contrast of scores on the change detection task in Missions 1 and 2.

This pattern of results would indicate that while simply increasing the number of updates from the robot may increase scores on the measures SA and human assistance provided to the robot, the updates may provide too many interruptions from the robot and hinder the ability of the human to complete his/her individual tasks.

\section{Effects of VPT on change detection task performance}

Non-significant differences on scores of dual task performance (i.e., the change detection task) were expected between the Traveling + waypoint updates with Level 1 VPT condition and the Traveling + waypoint updates with Level 2 VPT condition (i.e., Missions 3 and 4). Such that, scores on the change detection task for Mission 3 and Mission 4 were expected to be roughly equivalent (see Figure 38). 


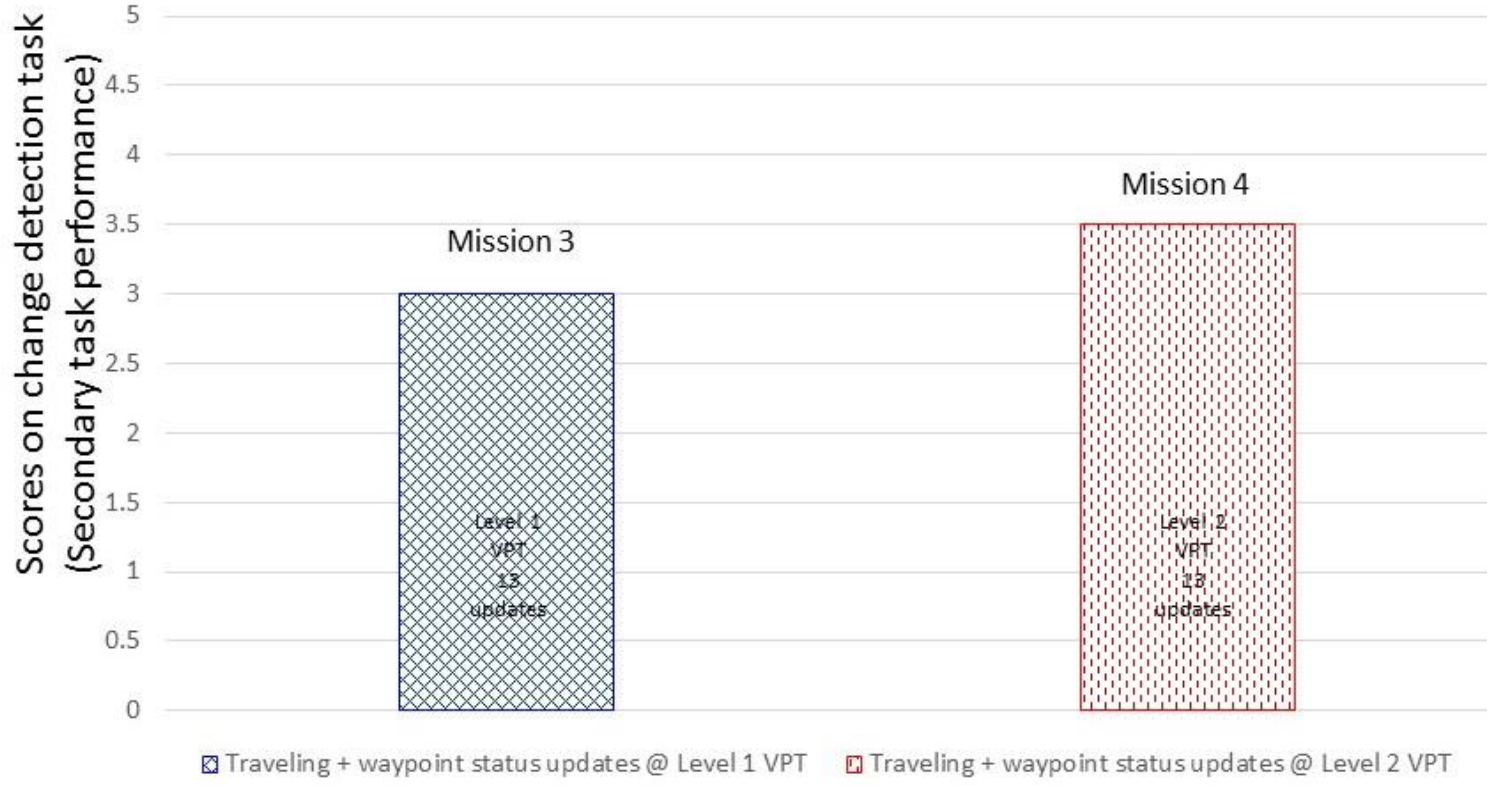

Figure 38. Contrast of scores on the change detection task in Missions 3 and 4.

Non-significant differences in change detection task performance were expected between the Waypoint status updates (B) condition (i.e., Mission 2) and the Traveling + waypoint updates with Level 1 VPT condition (i.e., Mission 3). Such that, scores on the change detection task for Mission 2 and Mission 3 were expected to be roughly equivalent (see Figure 39). 


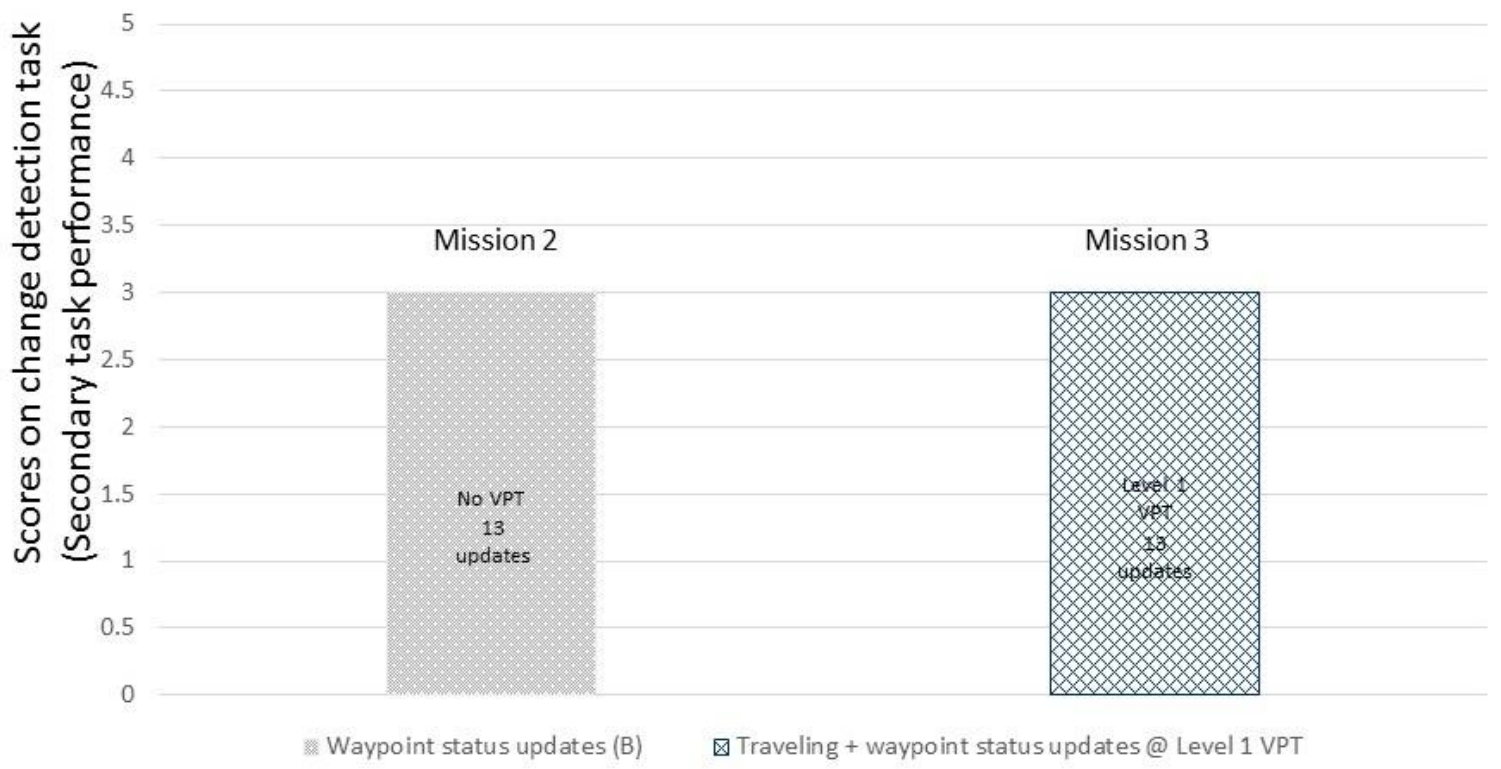

Figure 39. Contrast of scores on the change detection task in Missions 2 and 3.

In addition, scores on the change detection task were expected to be higher in Mission 4 than in Mission 3. It was anticipated that higher levels of VPT context added to robot-tohuman information exchanges would aid participants in spatial processing needed to maintain SA regarding the robot and its operating environment. As a result, additional cognitive resources may be freed up to devote to the dual change detection task (see Figure 40). 


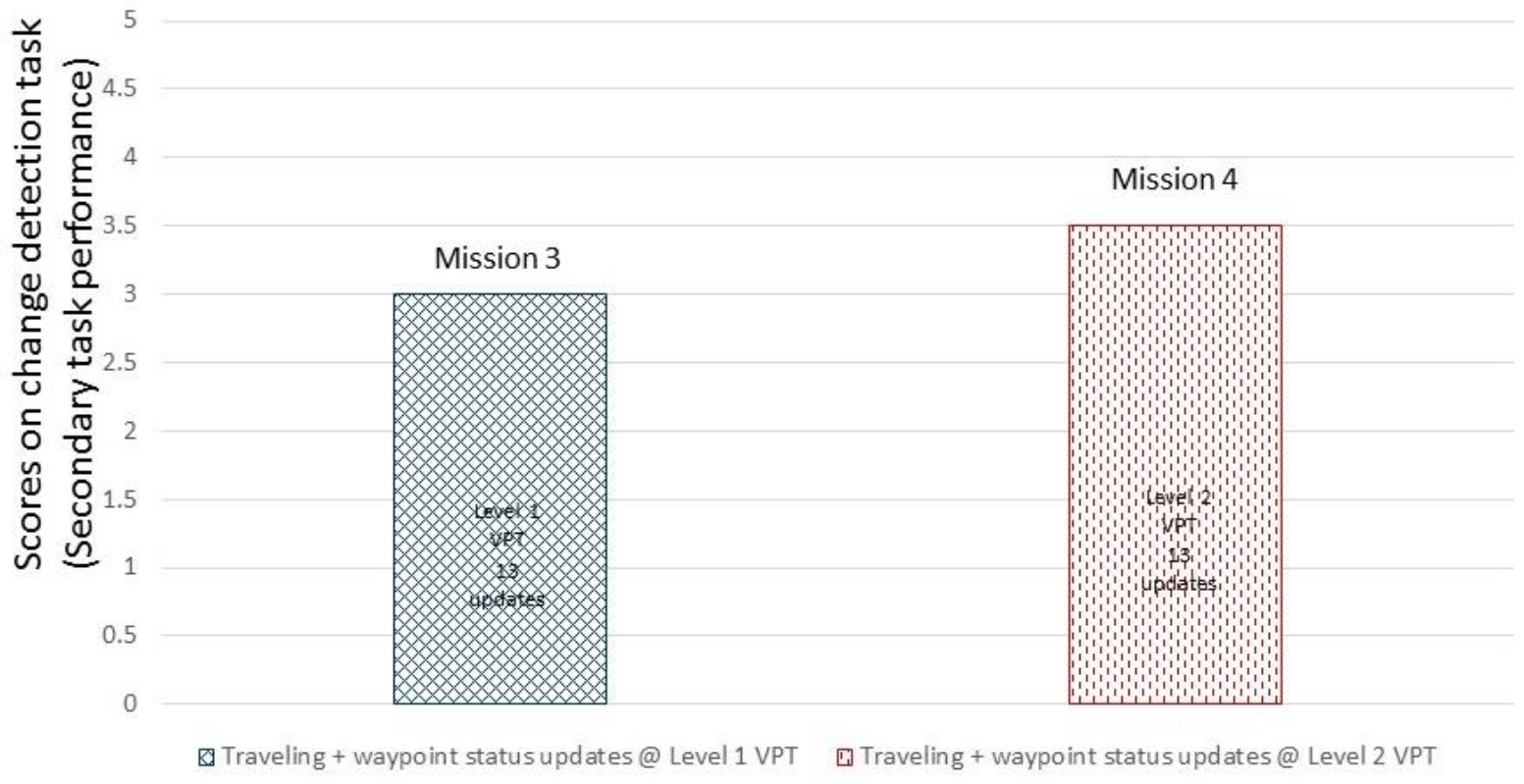

Figure 40. Contrast of scores on the change detection task in Missions 3 and 4.

Overall patterns of results

Figures 41, 42, and 43, give conceptual representations of the overall expected patterns of results for each condition on SA regarding the robot, human assistance provided to the robot, and change detection task performance. 


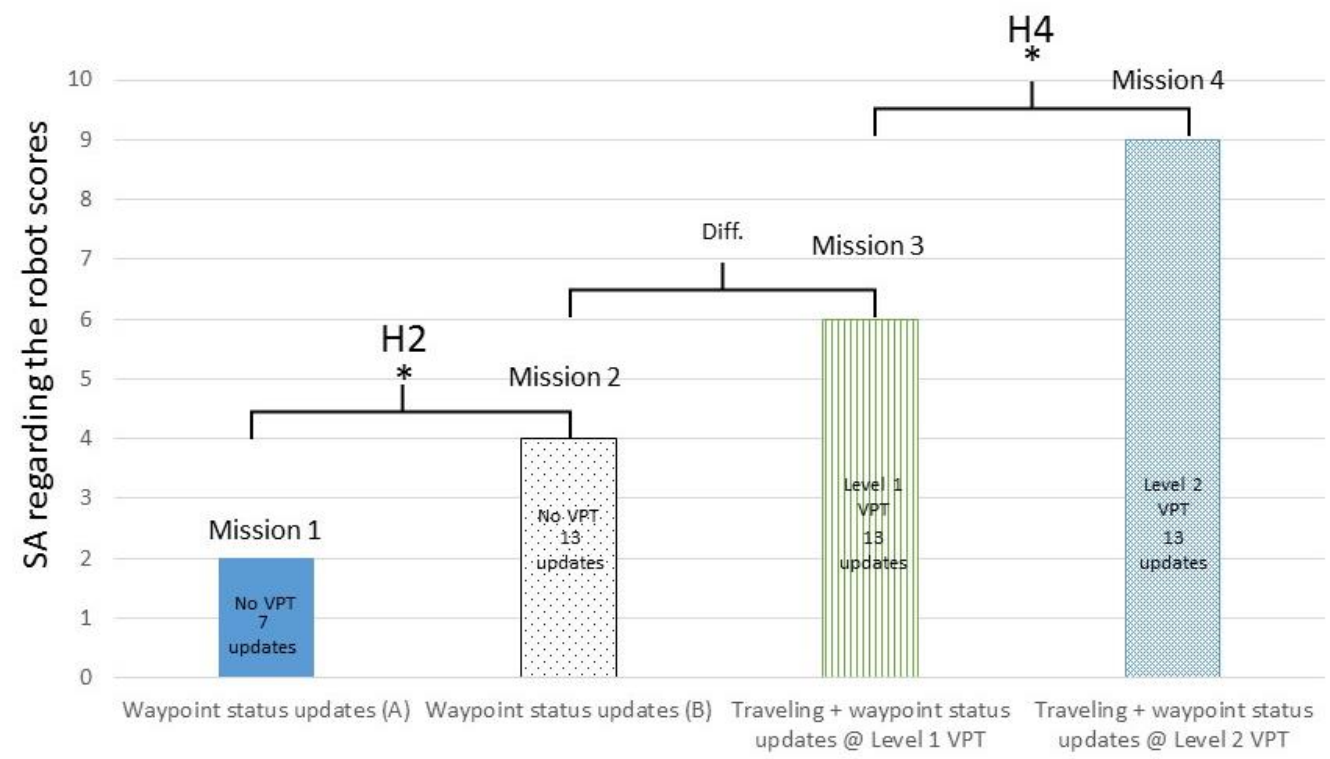

Figure 41. Conceptual representation of overall pattern of results for scores on the measure of SA regarding the robot.

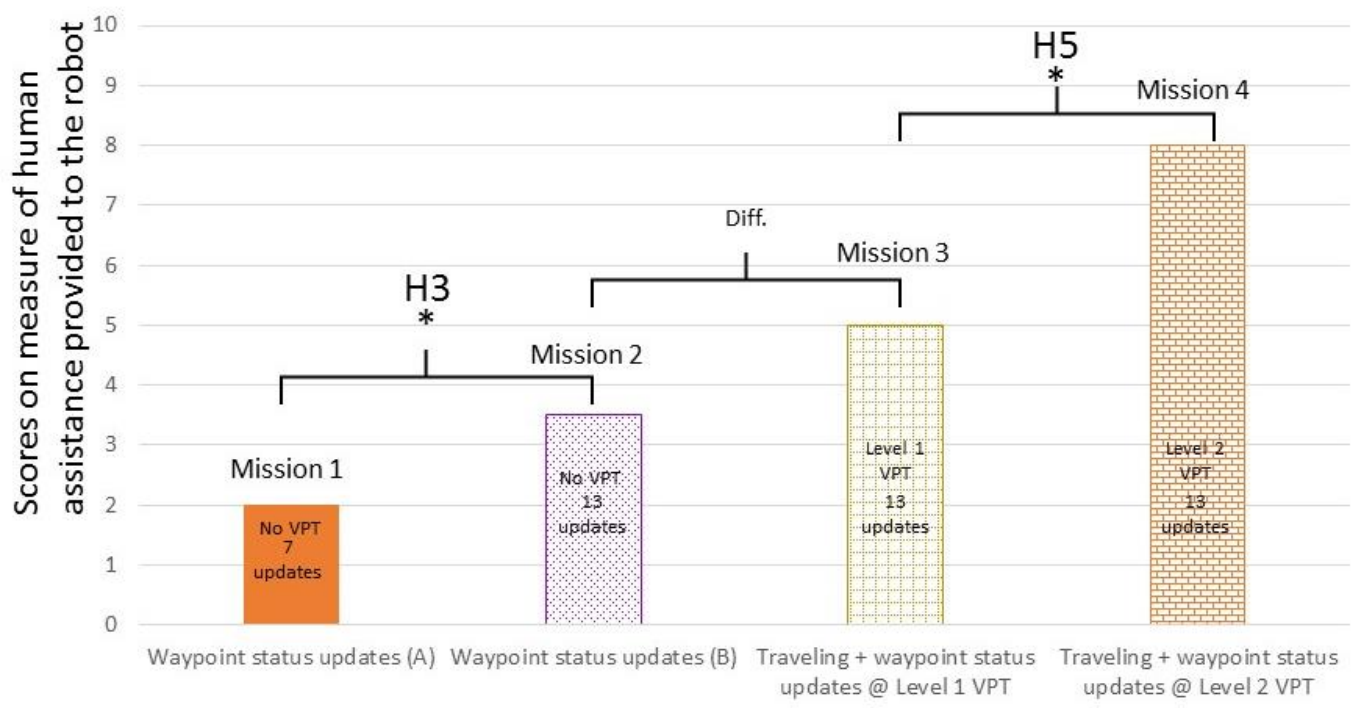

Figure 42. Conceptual representation of overall pattern of results for scores on the measure of human assistance provided to the robot. 


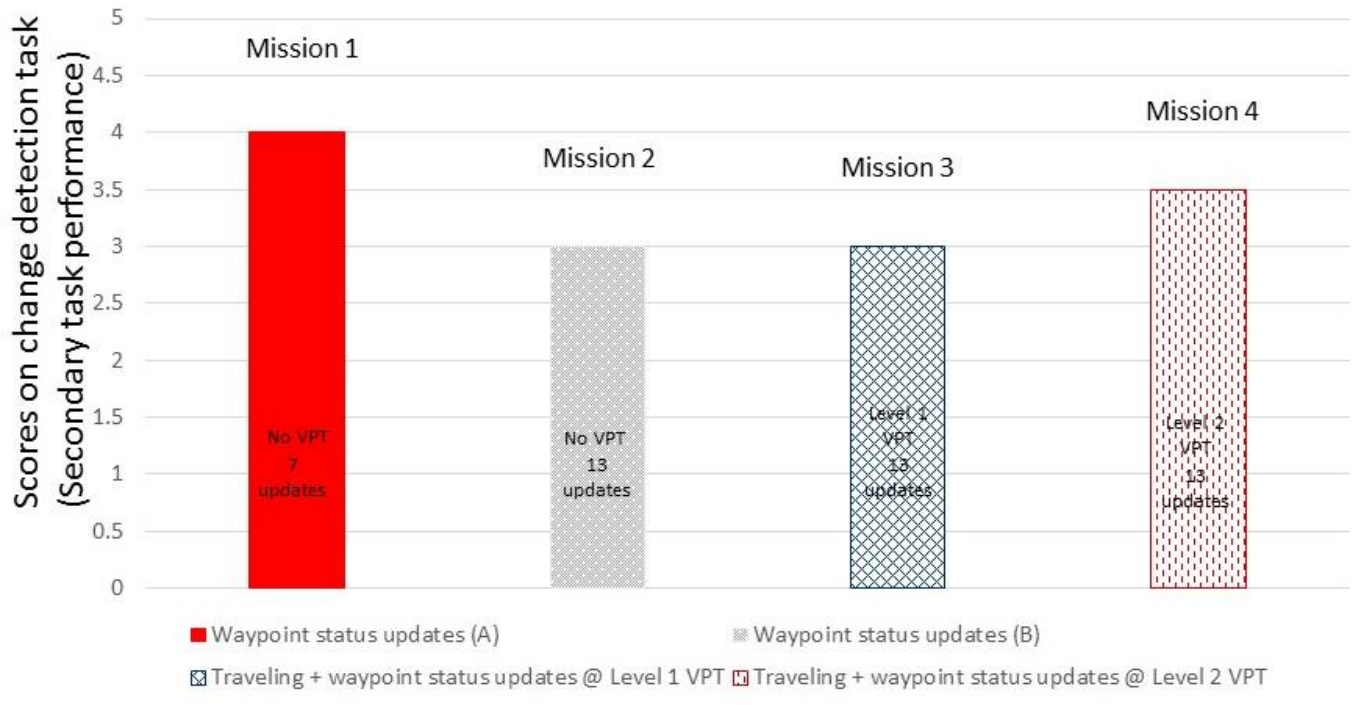

Figure 43. Conceptual representation of overall pattern of results for scores on the change detection task.

Relationship between SA regarding the robot and human assistance provided to the robot

SA regarding the robot was also expected to be predictive of human assistance provided to the robot, such that higher scores on the measure of SA regarding the robot were predicted to lead to higher scores on the measure of human assistance provided to the robot (see Figure 44). The pattern of results depicted in Figure 31 would support Hypothesis H1. 


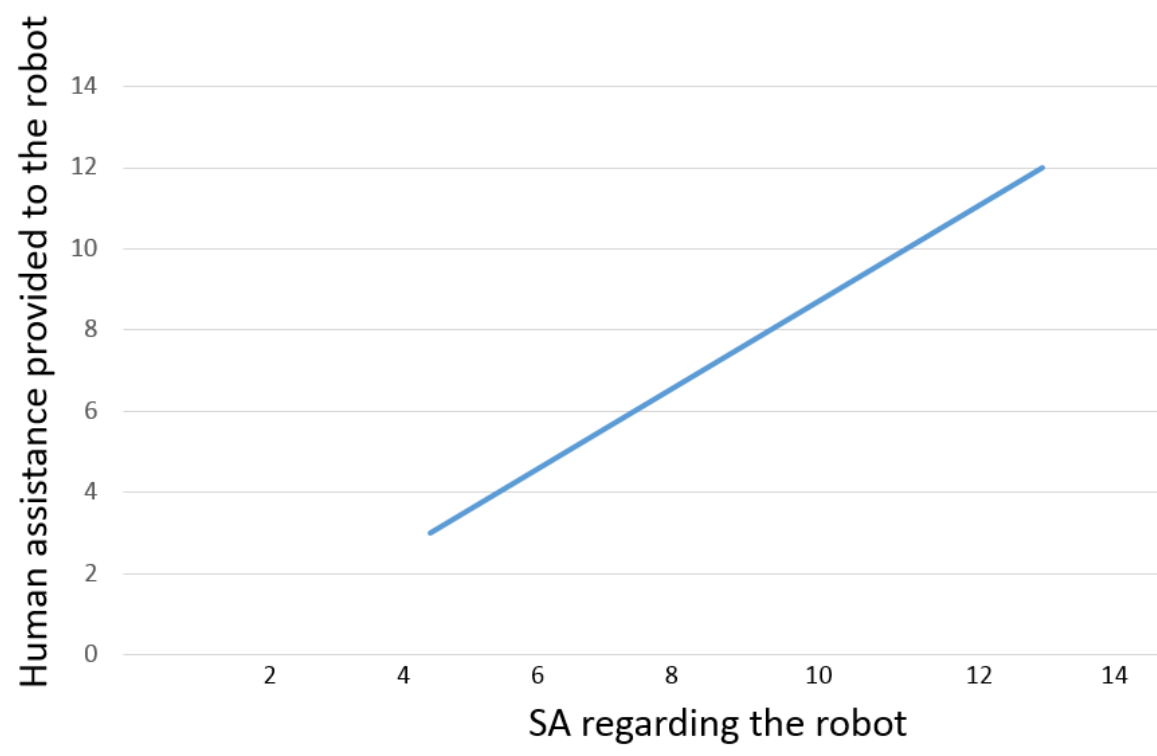

Figure 44. Expected positive relationship between SA regarding the robot and human assistance provided to the robot. 


\section{APPENDIX I: REMOVAL OF FEMALES IN THE ANALYSES}


The original analysis plan for the current study include running a series of oneway repeated measures ANCOVAs, with scores on the dependent measures (SA and providing assistance to the robot) as the within subjects variables, and scores on the measure of perspective taking ability (VPT) as the covariate. When the first ANCOVA was used to test for significant differences in SA scores across study conditions. The test showed a significant main effect for the within-subjects study condition, Wilks Lambda $=0.709, \mathrm{~F}(3,72)=9.845, \mathrm{p}<.001$. However, checks for the assumptions of the ANCOVA test revealed a significant interaction between the covariate and the within-subjects condition (i.e., the levels of treatment). Such that, a significant interaction between participants' scores on the measure of individual perspective taking ability and scores on the measures of SA across study conditions was present, Wilks Lambda $=0.868$, $F(1,74)=3.636, p=.01$. This finding revealed a violation of the assumption of homogeneity of regression slopes, suggesting the relationship between VPT scores and SA scores varied across study conditions. Gender ${ }^{7}$ was suggested as a variable that could provide a potential explanation for this relationship, as participant gender was significantly correlated with SA scores and VPT scores, Pearson's $r=-.313, p<.001$ (see table 14). Also, there were significant differences between the genders on the measure of perspective taking ability where, females $(M=63.50)$ did significantly worse than males $(M=36.48), t(74)=2.840, p=.006$.

\footnotetext{
${ }^{7}$ In this study gender actually refers to participants' biological sex as participants were not asked
} to indicate their gender identity. Rather, it was the intent of the researcher to ask participants to indicate their biological sex. 
Table 14. Table of Pearson's correlations $(N=76)$ between individual difference variables and dependent variables for both males and females in the study.

\begin{tabular}{|c|c|c|c|c|c|c|c|}
\hline Measure & 1. & 2. & 3. & 4. & 5. & 6. & 7. \\
\hline 1. Gender & 1 & & & & & & \\
\hline $\begin{array}{l}\text { 2. Corsi block } \\
\text { tapping task }\end{array}$ & .203 & 1 & & & & & \\
\hline $\begin{array}{l}\text { 3. Perspective } \\
\text { taking/Spatial } \\
\text { orientation test }\end{array}$ & $-.313 * *$ & $-.238^{*}$ & 1 & & & & \\
\hline $\begin{array}{l}\text { 4. Familiarity } \\
\text { with robots }\end{array}$ & .197 & -.051 & -.105 & 1 & & & \\
\hline $\begin{array}{l}\text { 5. Experience } \\
\text { with robots }\end{array}$ & -.017 & -.082 & $-.238^{*}$ & $.851 * *$ & 1 & & \\
\hline $\begin{array}{l}\text { 6. SA } \\
\text { regarding the } \\
\text { robot score }\end{array}$ & $.368 * *$ & .177 & $-.332 * *$ & .014 & -.080 & 1 & \\
\hline $\begin{array}{l}\text { 7. Providing } \\
\text { assistance to } \\
\text { the robot score }\end{array}$ & .098 & .126 & $-.354 * *$ & 052 & .069 & $.560 * *$ & 1 \\
\hline
\end{tabular}

To examine this interaction further, a 2(gender) x 4 (SA scores) ANCOVA with participant gender as the between subjects variable, scores on the measures of SA as the within subjects variables, and scores on the measure of VPT ability as the covariate was conducted. The interaction between SA scores and gender was not significant Wilks Lambda $=0.992, F(3,71)=0.180, p=.910$. The main effect of the within subjects variable (i.e., SA scores for each study condition) was significant, Wilks Lambda $=0.747$, 
$F(3,71)=8.028, p<.001$, suggesting that there were statistically significant differences in scores on the measure of SA between study conditions. The main effect of gender was also significant, Wilks Lambda $=0.868, F(1,74)=3.636, p=.017$. An investigation of the pattern of means reveled that females in the sample under scored males on the measure of SA across the within-subjects study conditions (see table 15). In addition, the interaction between the covariate and the within-subjects condition was still significant, Wilks Lambda $=0.877, F(3,71)=3.308, p=.025$.

Table 15. Mean scores for males and females on the measure of SA across experimental mission scenarios.

\begin{tabular}{|c|c|c|c|c|c|c|c|}
\hline \multicolumn{2}{|c|}{$\begin{array}{c}\text { 7UP } \\
\text { Condition }\end{array}$} & \multicolumn{2}{|c|}{$\begin{array}{c}\text { 13UP } \\
\text { Condition }\end{array}$} & \multicolumn{2}{|c|}{$\begin{array}{c}\text { 13UPLevel } 1 \\
\text { Condition }\end{array}$} & \multicolumn{2}{|c|}{$\begin{array}{c}\text { 13UPLevel } 2 \\
\text { Condition }\end{array}$} \\
\hline$M$ & $M$ & $M$ & $M$ & $M$ & $M$ & $M$ & $M$ \\
\hline Male & Female & Male & Female & Male & Female & Male & Female \\
\hline$\overline{8.716}$ & 7.804 & $\overline{7.819}$ & 6.480 & $\overline{7.414}$ & 5.991 & $\overline{6.210}$ & 4.561 \\
\hline
\end{tabular}

However, the inclusion of gender as a between-subjects variable into the ANCOVA model reduced the significance of the interaction between the VPT ability (the covariate) and scores on the measure of SA across study conditions (the treatment), from $p=.01$ to $p=.025$, suggesting that a mediating relationship between gender, VPT scores, and SA scores might be present.

To test this assumption, a hierarchical multiple regression was conducted with SA scores as the dependent variable. Participant gender was entered at step 1, and scores on the VPT measure were entered at step 2. In step one, gender explained a significant 
amount of variance in SA scores, $R^{2}=.135$, beta $=6.693, p=.001$. In step two, the amount of variance in SA scores explained by the overall model with both predictors (i.e., VPT ability scores and gender), was also significant, $R^{2}=.188, p=.033$, and the addition of VPT scores in the model represented a significant change in the amount of variance explained by the model $R^{2}$ change $=.052, p=.033$. In step 2 , both participant gender and scores on the VPT ability measure were uniquely significant predictors of SA scores, $b e t a=5.320, p=.010$, and beta $=-0.051, p=.033$ respectively. However, after VPT scores were added at step 2, the amount of variance in SA scores uniquely explained by gender was reduced from beta $=6.693, p=.001$ to beta $=-5.320, p=.010$.

These findings suggest that in the prediction of SA scores for this study, gender was an important influencing factor. However, after controlling for gender, VPT ability was also important in predicting SA scores. It seems that some of the variation in SA scores may be attributed to gender, but some may also be attributed to individual differences in VPT ability. To isolate the effect of VPT ability on the dependent measures of interest in this study and to and aid in interpreting the analyses of the study hypotheses, the analyses conducted to test hypotheses H1-H5 were conducted with only the male participants obtained from the sample, $N=56$, ages ranging from $18-29(M=$ $18.89, S D=3.412)$. 


\section{APPENDIX J: INFORMED CONSENT}


University of

Central

Florida

\section{Supporting situation awareness through robot-to-human information exchanges under conditions of visuospatial perspective taking \\ Informed Consent}

Principal Investigator(s): Elizabeth Phillips

Sub-Investigator(s): Andrew Talone (Graduate research assistant)

Karissa Kasper (Research assistant)

Lauren Haskin (Research assistant)

MiAmor Aguirresaenz (Research assistant)

Nastacia Smith (Research assistant)

Nathan Zhang (Research assistant)

Samuel Resende (Research assistant)

Faculty Supervisor: Florian Jentsch $\mathrm{PhD}$

Sponsor: This research is being funded by the Department of Defense, Army Research Lab, and General Dynamics

Investigational Site(s): University of Central Florida

Main Campus

4000 Central Florida Blvd. Orlando, Fl 32817

Psychology Building Room 303G

OR

Institute for Simulation \& Training Team Performance Lab

Partnership II Building room 117A

3100 Technology Parkway Orlando, Fl 32826

1 of 6

University of Central Florida IRB

University of Central Florida 18
IRB NUMBER: SBE-15-11395

IRB APPROVAL DATE: 07/06/2015

IRB EXPIRATION DATE: 07/05/2016 


\section{Introduction:}

Researchers at the University of Central Florida (UCF) study many topics. To do this we need the help of people who agree to take part in a research study. You are being invited to take part in a research study which will include about 99 people at UCF. You have been asked to take part in this research study because you are an undergraduate student enrolled in a psychology class at UCF.

Please note: You must be 18 years of age or older to be included in this research study. In addition, in order to participate in this study you must NOT have prior history of experiencing seizures. For your protection, if you have experienced seizures in the past, please notify the study experimenter immediately. You will not be penalized for withdrawing from participation due to this exclusion.

The people doing this research are Elizabeth Phillips, Andrew Talone, and Florian Jentsch of the College of Sciences at UCF. Because some of the researchers are graduate students, they are being guided by Dr. Florian Jentsch, a UCF faculty supervisor in the Department of Psychology. UCF students learning about research are also helping to do this study as part of the research team. Their names are: Karissa Kasper, Lauren Haskin, MiAmor Aguirreseanz, Nastacia Smith, Samuel Resende, and Nathan Zhang.

What you should know about a research study:

- Someone will explain this research study to you.

- A research study is something you volunteer for.

- Whether or not you take part is up to you.

- You should take part in this study only because you want to.

- You can choose not to take part in the research study.

- You can agree to take part now and later change your mind.

- Whatever you decide it will not be held against you.

- Feel free to ask all the questions you want before you decide.

\section{Purpose of the research study:}

The objective of this study is to test the effects of information exchanges provided by a robot on the development of SA in a human partner, under differing levels of visual perspective taking, and their consequential effects on robot aiding behavior in a simulated human-robot team task. We plan to use the results of this study to inform the design and engineering community on ways to implement proactive information sharing behaviors in robots. 
What you will be asked to do in the study:

To begin the study, you will be asked fill out a series of biographical and experience questionnaires, as well as individual ability measures. Then you will be familiarized with the nature of the tasks that you will be asked to complete through a series of training presentations and practice scenarios. You will then be asked to complete 4 experimental scenarios. During completion of these scenarios, you will be asked to play a Soldier in a simulated Soldier-robot team. You will be asked to receive and understand updates sent to you from a simulated robot. Use the information in the updates to answer Situation Awareness related questions concerning the robot's location in a simulated urban city environment, and then make adjustments to the simulated robot's waypoint path through the environment. In addition, you will be asked to monitor a change detection task in which they will indicate when a series of icons presented in the MIX testbed, appear, disappear, or move across the screen.

Once experimental scenarios are completed you will be asked to fill out another series of questionnaires and surveys. Once these have been filled out, you will be given debriefing information regarding the nature of the study and it's purposes as well as contact information for the research team, principal investigator, faculty adviser, and internal review board should you wish to find out about the results of the study in the future or if you would like to address general comments or concerns. In addition, you will be given the opportunity to evaluate the research team on their performance and treatment of you as a research participant. The completion of this evaluation form is entirely optional, however.

A general description of the course of the study is below.

Study Timeline and estimated time to completion

\section{Phase 1: Background and Training}

1. Biographical data and prior knowledge of robots measure $5 \mathrm{~min}$

2. Working memory measure $10 \mathrm{~min}$

3. Visuospatial ability measure $\quad 10 \mathrm{~min}$

4. Mental model drawing measure $5 \mathrm{~min}$

5. Shared mental model measure ( 1 of 2 ) 5 min

6. Training PowerPoint $30 \mathrm{~min}$

7. Practice missions $10 \mathrm{~min}$

Phase 2: Experiment

8. Missions 1-4 $40 \mathrm{~min}$

\section{Phase 3: Post participation}
9. Shared mental model measure ( 2 of 2 ) 5 min
10. Post participation information form $5 \mathrm{~min}$
11. [Optional] Researcher evaluation form $5 \mathrm{~min}$

Anticipated time to completion: $130 \mathrm{~min}$. (2 hours and 10 minutes)

2 hours and 10 minutes will be rounded to 2 hours and 30 minutes for SONA credit.

3 of 6

University of Central Florida IRB

University of Central Florida IR
IRB NUMBER: SBE-15-11395

IRB APPROVAL DATE: 07/06/2015

IRB EXPIRATION DATE: 07/05/2016 
Please note that you do not have to answer every question or complete every task. You will not lose any benefits if you skip questions or tasks.

\section{Location:}

The research will be conducted at either the Psychology building at UCF room $303 \mathrm{G}$ or the UCF Institute of Simulation and Training Partnership II building located at

3100 Technology Parkway, room 117 A

Orlando, 32826

\section{Time required:}

We expect that you will be in this research study for up to approximately 2 hours and 10 minutes.

\section{Funding for this study:}

This research study is being paid for by The Army Research Laboratory and General Dynamics as a part of the Robotics Collaborative Technology Alliance (RCTA).

\section{Risks:}

Researchers believe that the likelihood of participant risk is very low. However, there may be risks of side effects associated with prolonged computer usage including eye strain, dry eye, or fatigue. However, these risks are no greater than the risks participants may be exposed to when using the computer for other purposes. To minimize these effects, you will be permitted to take breaks as you feel necessary by notifying the experimenters throughout the course of the study.

Further, there may be concern that the suggestion of a military scenario or a robotic teammate may invoke a negative response to those sensitive to issues associated with military conflict, police investigation, crime, or artificial intelligence, or participation in research that is funded by the U.S government, Department of Defense, or the U.S. Army. Other risks associated with participation in this research study are unforeseeable.

\section{Benefits:}

We cannot promise any benefits to you or others from your taking part in this research. Participants will be immersed in an environment of scholarly research which may help to augment research education.

\section{Compensation or payment:}

Participants may expect to spend approximately 130 minutes performing experimental tasks, for which they may receive Sona Systems credit for the amount of time they participate. Maximum Sona Systems credit will be 2.5 credits, the equivalent of 150 minutes. There is no direct compensation for taking part in this study. It is possible, however, that extra credit may be offered for your participation, but this benefit is at the discretion of your course instructor.

\section{Confidentiality:}

4 of 6

University of Central Florida IRB

IRB NUMBER: SBE-15-11395

IRB APPROVAL DATE: 07/06/2015

IRB EXPIRATION DATE: 07/05/2016 
We will limit your personal data collected in this study to people who have a need to review this information. However, due to the requirement to come into the research facility, we cannot promise complete secrecy. Additionally, you are required to sign the Informed Consent document. This document will not be attached to the data we collect from you, however we will still be in the possession of the Informed Consent document which may have to be disclosed to the IRB. In addition, because this research is sponsored by the Department of Defense and the U.S. Army, the Army Human Research Protections Office is eligible to review the research records. Lastly, only de-identifiable summary results of this study will appear in research reports which may include, but are not limited to, technical reports and scholarly per reviewed journal articles and conference proceedings.

\section{Study contact for questions about the study or to report a problem:}

If you have questions, concerns, complaints, or think the research has hurt you in anyway, please feel free to contact Elizabeth Phillips, a graduate student in the Applied Experimental and Human Factors Psychology $\mathrm{PhD}$ program or, the principal investigator and faculty supervisor Dr. Florian Jentsch in the Department of Psychology. Their contact information is as follows:

\section{Elizabeth Phillips}

ephillip@ist.ucf.edu

AEHF PhD program

Department of Psychology and Institute for Simulation and Training, UCF

Dr. Florian Jentsch (Principal Investigator \& Faculty Adviser)

Florian.Jentsch@ucf.edu

Department of Psychology and Institute for Simulation and Training (407)882-0304

\section{IRB contact about your rights in the study or to report a complaint:}

Research at the University of Central Florida involving human participants is carried out under the oversight of the Institutional Review Board (UCF IRB). This research has been reviewed and approved by the IRB. For information about the rights of people who take part in research, please contact: Institutional Review Board, University of Central Florida, Office of Research \& Commercialization, 12201 Research Parkway, Suite 501, Orlando, FL 32826-3246 or by telephone at (407) 823-2901. You may also talk to them for any of the following:

- Your questions, concerns, or complaints are not being answered by the research team.

- You cannot reach the research team.

- You want to talk to someone besides the research team.

- You want to get information or provide input about this research.

Withdrawing from the study:

Participation in this research study is completely voluntary. Refusal to participate or choosing to withdraw from the study at any time will involve no penalty or loss of benefits associated with the study. If you decide to leave the research study, you will receive credit in accordance with time spent in the study prior to withdrawal. For example, if you decide to withdrawal from the study after 1 hour has passed, you will receive 1 Sona credit. If you decide to leave the study, contact the investigator so that the investigator can stop administering the survey instruments or stop the simulation. The

5 of 6

University of Central Forida IRB IRB NUMBER: SBE-15-11395 
researcher will then thank you for your time, explain how you will be credited for your time, and instruct you to the exit. The person in charge of the research study or the sponsor can remove you from the research study without your approval. Possible reasons for removal include failure to follow instructions of the research staff, disorderly conduct, improper treatment of the research staff, other participants, research equipment, or if the research staff feels that the study is no longer in your best interests. We will tell you about any new information that may affect your health, welfare or choice to stay in the research.

Your signature below indicates your permission to take part in this research.

DO NOT SIGN THIS FORM AFTER THE IRB EXPIRATION DATE BELOW

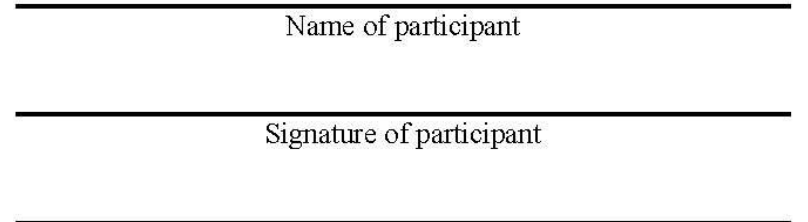

Signature of person obtaining consent

Printed name of person obtaining consent

\begin{tabular}{c}
\hline Date \\
\hline Date
\end{tabular}


APPENDIX K: POST-PARTICIPATION INFORMATION 


\section{Post Participation Information}

Supporting situation awareness through robot-to-human information exchanges under conditions of visuospatial perspective taking

This study was designed to investigate effects or robot information sharing and the application of visuospatial perspective information on one's ability to develop and maintain situation awareness regarding a robot and the subsequent ability to aid a robot in a navigation task. We wish to use this information to inform the design and engineering community on designing robots that can proactively communicate with human teammates and the degree to which robot reporting behaviors can facilitate human-robot team performance.

We want you to know that we could not do our work without your help, so your participation is greatly appreciated. Please feel free to ask any questions at this time about the procedure or the study in general. Should you desire to learn more about the study or receive the results of the study when they become available, please contact the faculty advisor; Dr. Florian Jentsch.

All the data you have contributed to this study will be held in strict confidentiality by the researchers and will not be revealed to anyone other than the researchers and their immediate assistants. The primary investigator is Elizabeth Phillips (ephillip@ist.ucf.edu) and the faculty advisor is Dr. Florian Jentsch (florian.jentsch@ucf.edu). We ask that you please refrain from discussing the details of this study with other individuals as they may wish to participate in this study at a later time.

Thank you again for your participation.

Contact Information:

Office (Research Park):

Florian Jentsch

University of Central Florida

Director, Team Performance Laboratory, IST

Partnership II Building

UCF Department of Psychology 3100 Technology Parkway

Suite 117

Orlando FL 32826

Phone: 407-882-0304

E-mail: florian.jentsch@ucf.edu

\section{Contact information for UCF's IRB}

Institutional Review Board, University of Central Florida, Office of Research \& Commercialization, 12201

Research Parkway, Suite 501, Orlando, FL 32826-3246 or (407) 823-2901 


\section{APPENDIX L: PARTICIPANT TRAINING SLIDES}




\section{Experimental Training Tutorial}

US Army Research Laboratory

UCF Institute for Simulation \& Training

Fall 2015

ARL 15-067 UCF SBE 15-11395

ARL

\section{Background Information}

- The Department of Defense (DoD) is testing new deployable technology to be used by dismounted Soldiers during intelligence, surveillance, and reconnaissance (ISR) operations. New technologies include robots that are capable of full autonomy. Meaning they can be commanded to carryout a mission task without having to be within proximity of the rest of the

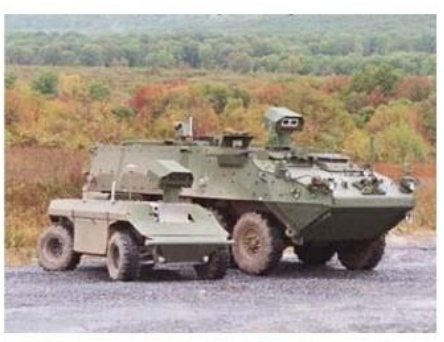
squad or need direct continuous supervision. 


\section{Background Information}

- This robotic technology is still in a testing phase. At this point there are technological and design issues that need to be addressed and we need your help to resolve these problems.

- The robot is fully equipped with a host of sensors that allow it to capture data from the environment in order to navigate over various terrain, maneuver around various obstructions, and identify objects and buildings, but the robot needs assistance in order to correctly navigate through these areas of deployment.

- The robot that will be used in this study will be simulated by computerized software that emulates the robot's sensing equipment.

\section{Mission}

Your role is to work together with the simulated robot on a reconnaissance and surveillance mission by completing the following main objectives.

\section{Main Objectives:}

1. Provide surveillance by monitoring changes to an area of interest in the environment.

2. Help the robot to move through an urban environment as quickly as possible while simultaneously avoiding sensitive areas in the environment.

3. Help the robot to learn alternate routes through the environment, in the event that it veers off course.

4. Work to complete the mission in a timely manner. 


\section{Your role}

You will be asked to:

- Play the role of a Soldier in a simulated Soldier-robot team.

- Provide surveillance of an area of interest and make note of changes to that area.

- Use updates from the robot to keep track of the robot's movement and answer questions regarding the robot's movement and location.

- Provide re-routing information that helps the robot to avoid sensitive areas, in the event that the robot veers off course.

- Pay attention to how quickly the robot is completing its objectives.

\section{The Robot}

The robot will:

- Provide reconnaissance by navigating a waypoint path through the environment.

- Periodically share updates with you regarding the buildings located at each waypoint, as well as other status information concerning its navigation.

- The following slide will provide an example of a robot's planned waypoint path through the environment. 


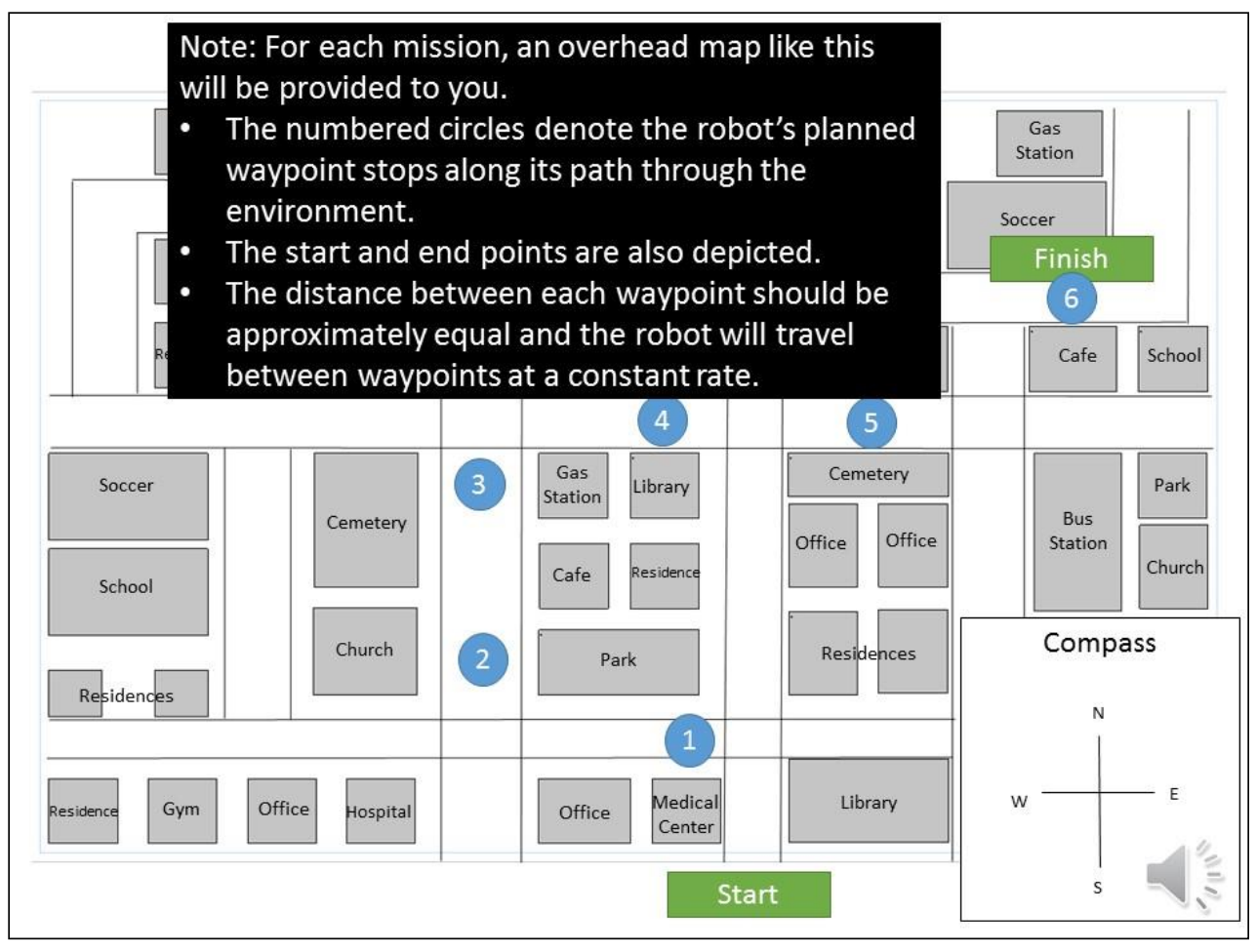

\section{Surveillance: Monitoring changes}




\section{Monitoring changes}

- Throughout the course of the study you will see an overhead view of an area of interest.

- You will be asked to monitor changes to icons displayed in this view.

- There are three types of changes: appear, disappear, and move

-When you observe a change, you will be asked to indicate whether the icons appeared, disappeared, or moved by clicking on the buttons displayed on the screen.

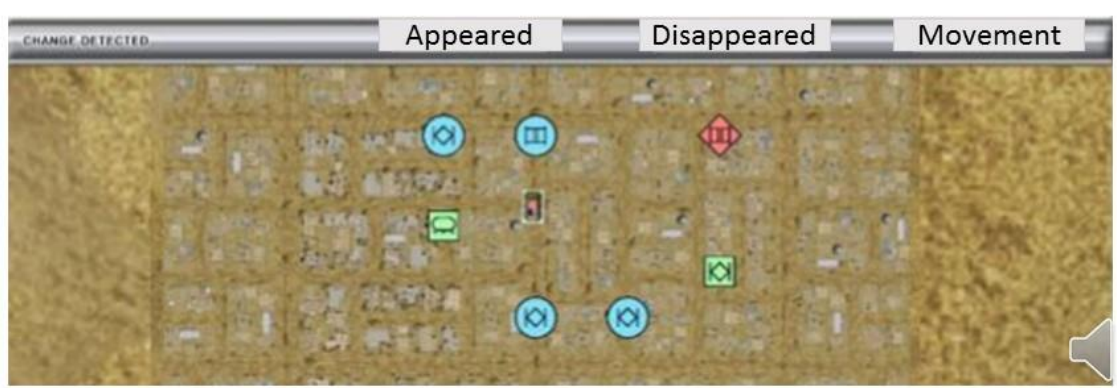

\section{Monitoring changes}

- For the "appear" changes, new icons will be added to the array of icons displayed on the screen.

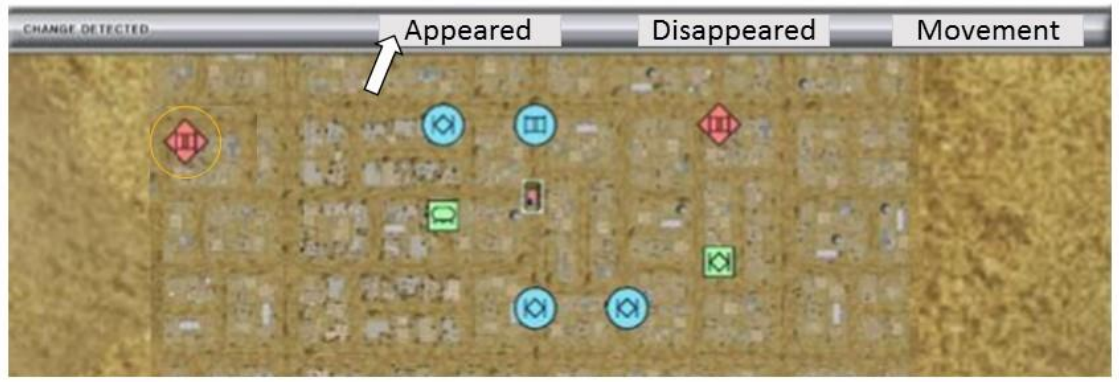

- Indicate that icons have appeared by using the mouse cursor to click the "Appeared" button. 


\section{Monitoring changes}

- For the "disappeared" changes, existing icons will leave the screen.

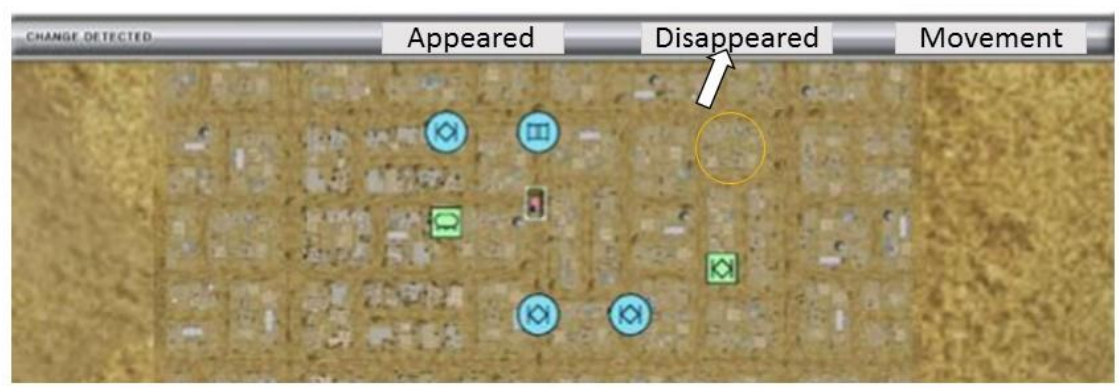

- Indicate that icons have disappeared by using the mouse cursor to click the "Disappeared" button.

\section{Monitoring changes}

- For the "movement" changes, existing icons will move from one position to another.

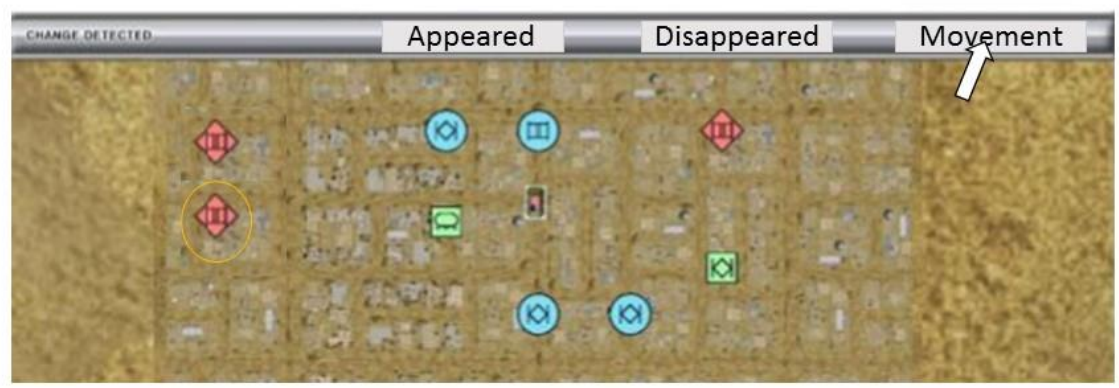

- Indicate that icons have moved by using the mouse cursor to click the "Movement" button. 


\section{STOP HERE}

- You will now practice the surveillance task by monitoring changes to the area of interest.

- If, after the following training, you feel like you need more practice, please let the experimenter know.

\section{Countdown clock}




\section{Countdown clock}

- Throughout the course of each mission, a countdown clock will be present.

- Each mission should take approximately 5 minutes to complete.

- The countdown will begin at the start of each mission. The robot is expected to reach it's final waypoint by the time the countdown clock reaches zero.

- You will be asked to keep an eye on this countdown to determine whether or not the robot is going to complete its waypoint path before the clock runs out.

\section{Reconnaissance: \\ Updates from the robot}




\section{Updates from the robot}

The robot will periodically share intelligence and reconnaissance information with you concerning:

- The status of the robot's waypoint navigation through the environment.

- Intelligence concerning the buildings located around each waypoint stop.

The robot will provide two types of updates. The following slides will provide examples of each type. They are

- Waypoint updates

- Traveling updates

\section{Updates from the robot}

Waypoint updates:

- When the robot reaches a waypoint, it will stop, scan the environment, and then provide information concerning the buildings located within its immediate area.

- The robot will also provide other status information.

- Waypoint updates will be given to you each time the robot stops at a waypoint along its path.

- The robot will always provide an update each time it stops. 


\section{Elements of the Waypoint update}

Waypoint Update from the robot

Status: Stopped/Scanning

Time to target: $3 \mathrm{~m} \mathrm{00s}$

Percent complete: $16 \%$

Intel: There is@church on one side and a park onthe other
- The robot is stopped.

- The robot's estimated time until it completes the waypoint route.

- Approximate percentage of the waypoint route the robot has completed.

- Intelligence concerning buildings surrounding the robot.

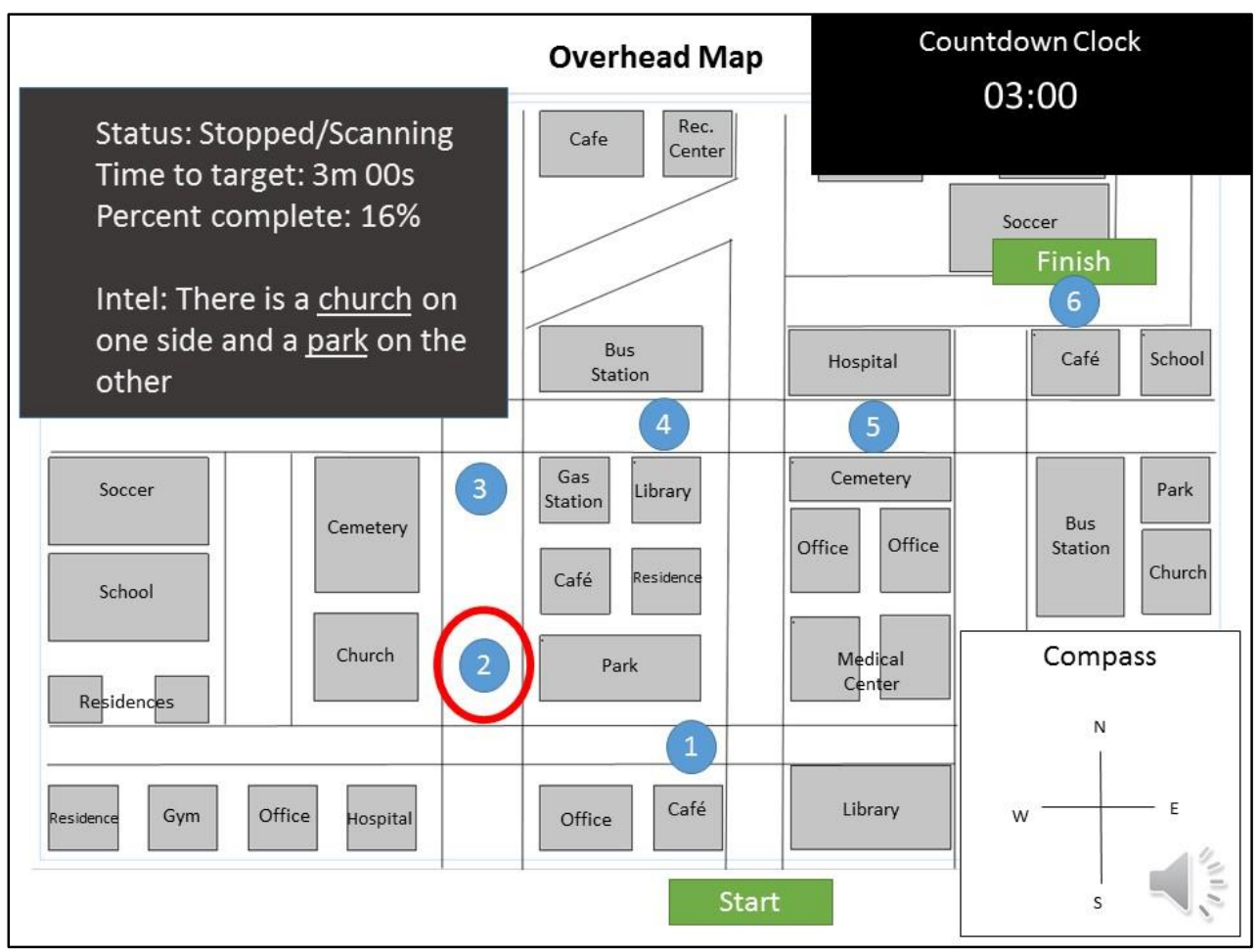




\section{Updates from the robot}

Traveling updates:

- Periodically, the robot will also share information with you while it is traveling from one waypoint to another.

- Traveling updates will provide you with information concerning the direction the robot is traveling.

- Because the robot is still in testing phases, the robot will provide traveling updates in some instances and not in others.

- In addition, the robot may be able to provide extra traveling information in some instances and not others.

\section{Elements of the Traveling updates}

Traveling Update from the robot

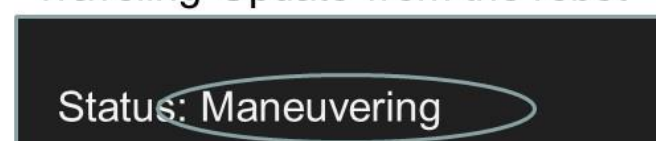

Heading: North

toward the next waypoint
- The robot is maneuvering between waypoints.

- The direction the robot is traveling

- The robot is traveling North towards its next waypoint. 


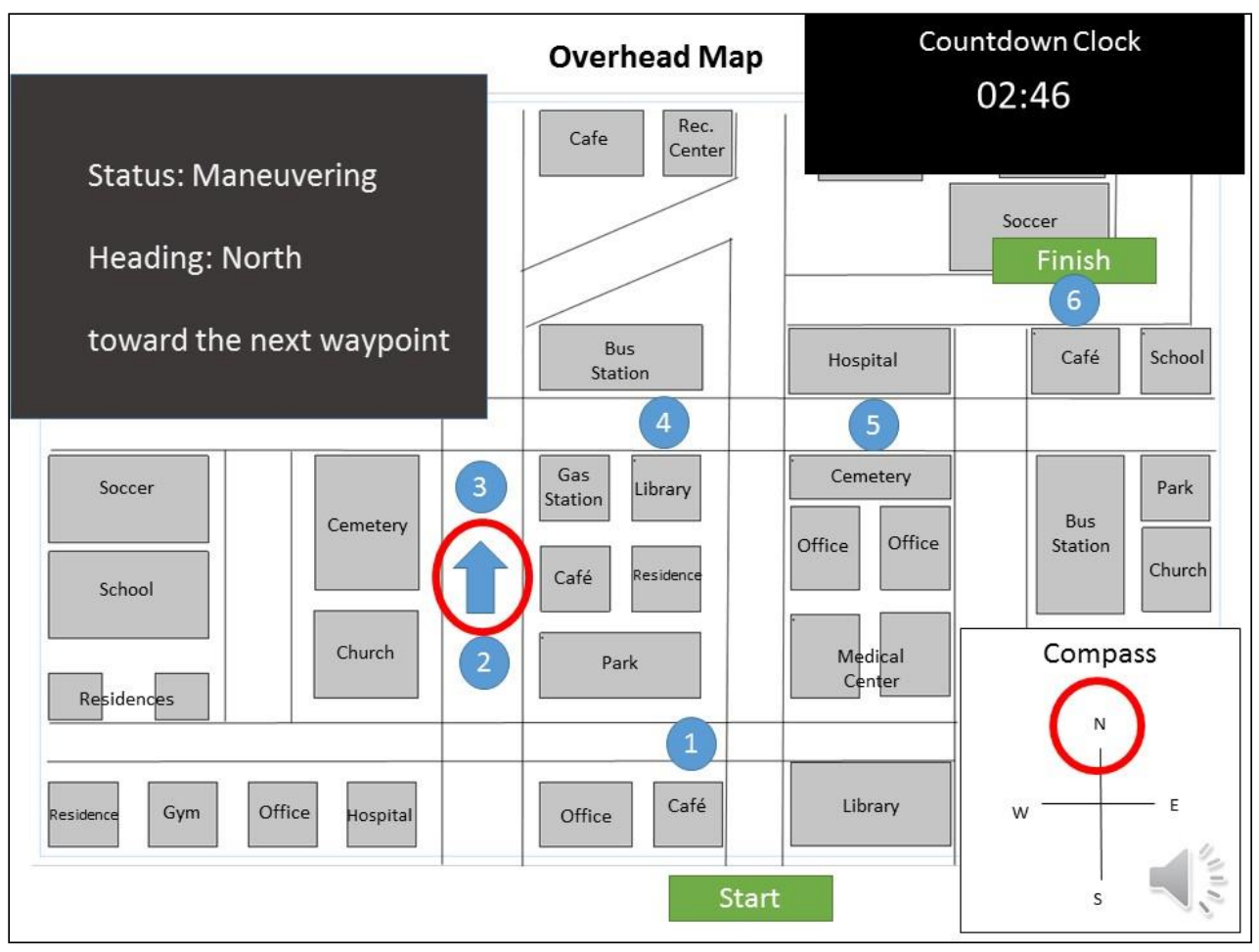

\section{The Compass rose}




\section{Compass rose}

- A compass rose will be depicted on the overhead map that shows the robot's planned waypoint path through the environment.

- A compass rose is figure on a map or chart used to display the orientation of cardinal directions (i.e., North, South, East, West) relative to the

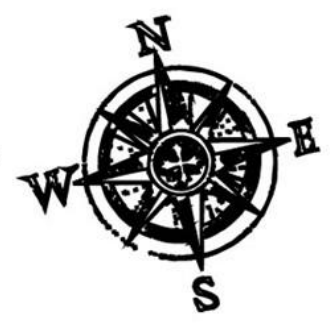
map or chat.

- The compass rose can also display intermediate directions as well as combination points.

\section{Compass rose}

Cardinal directions

- North (N)

- South (S)

- East (E)

- West (W)

Depicted at right angles, $90^{\circ}$

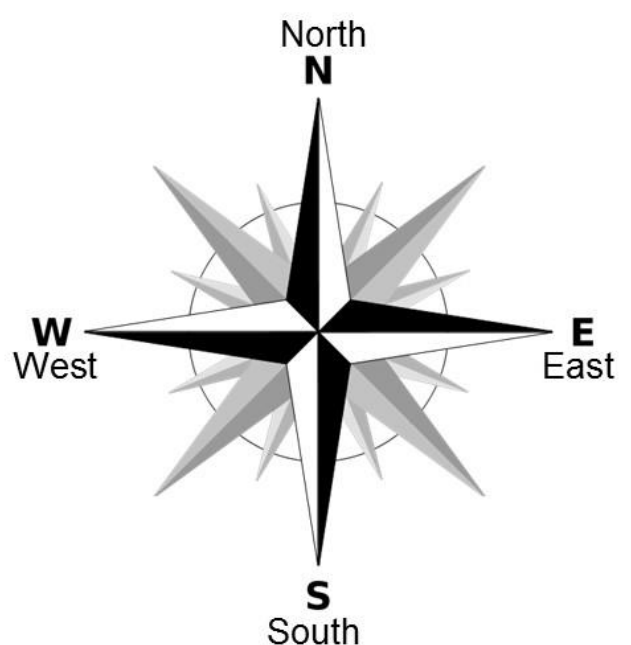




\section{Compass rose}

Intermediate directions

- Lie between each Northwest cardinal direction, $45^{\circ}$

- Northeast (NE)

- Northwest (NW)

- Southeast (SE)

- Southwest (SW)

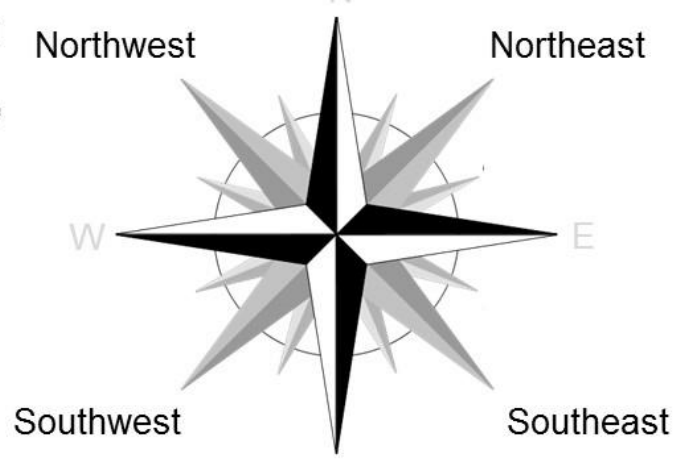

\section{Compass rose}

Combination points

- Lie between the Cardinal and Intermediate directions, Approx. $22^{\circ}$

-North-Northeast (NNE)

-East-Northeast (ENE)

-East-Southeast (ESE)

-South-Southeast (SSE)

-South-Southwest (SSW)

-West-Southwest (WSW)

-West-Northwest (WNW)

-North-Northwest (NNW)

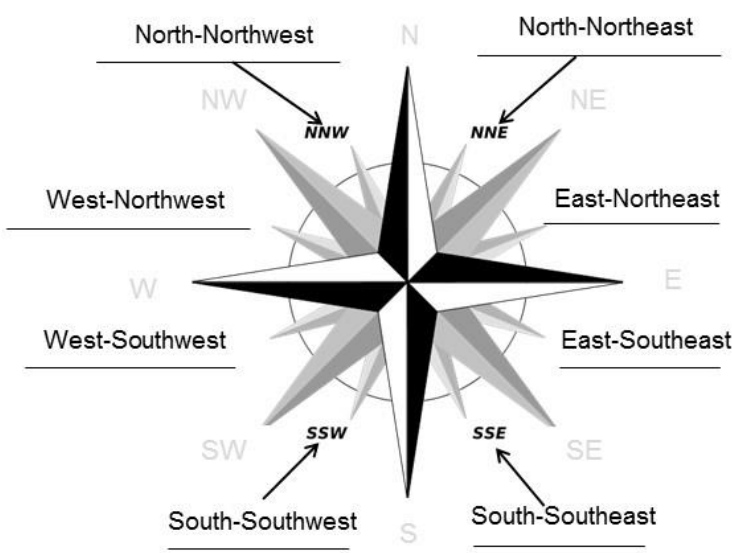

South-Southwest

South-Southeast 

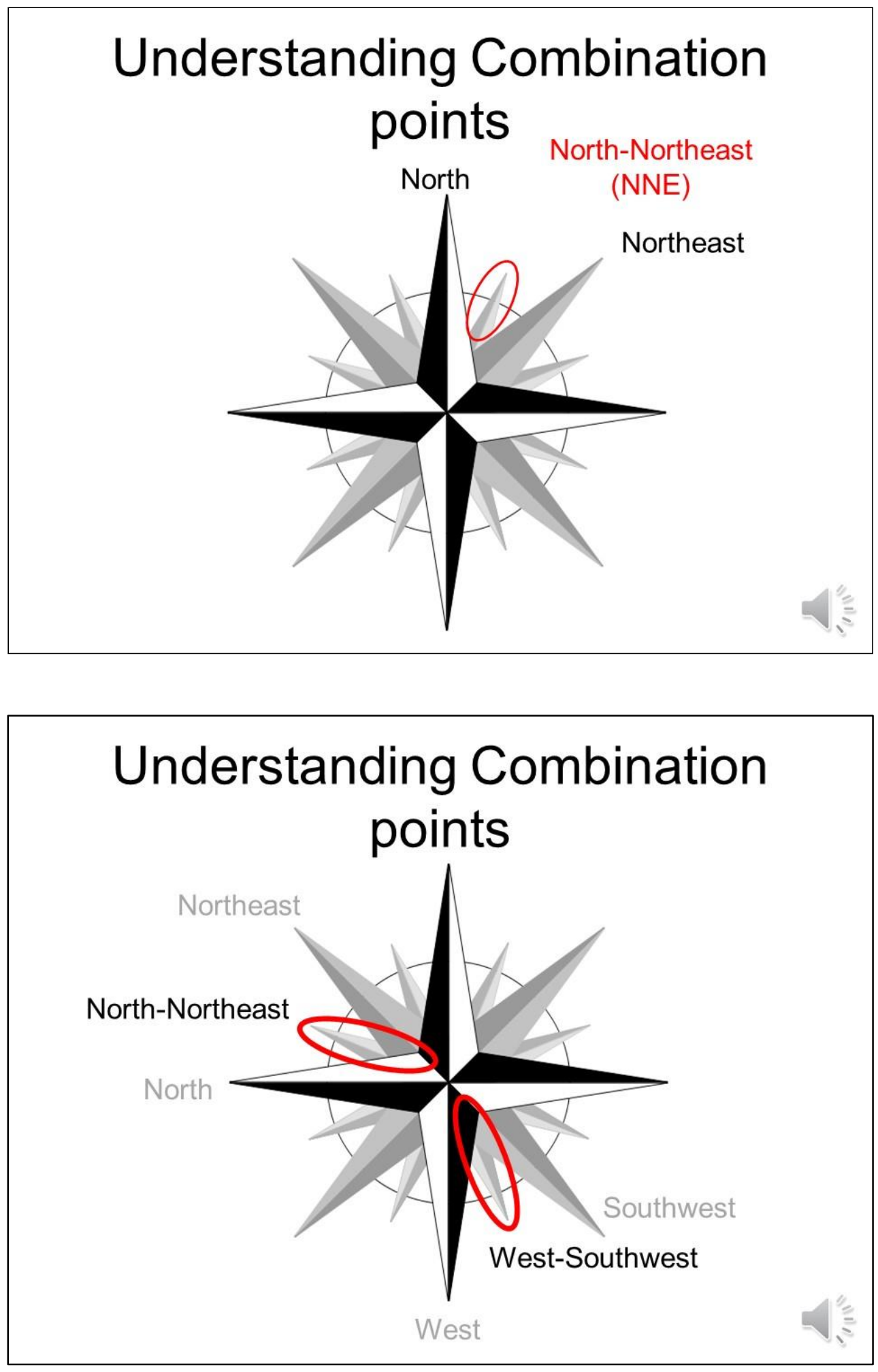

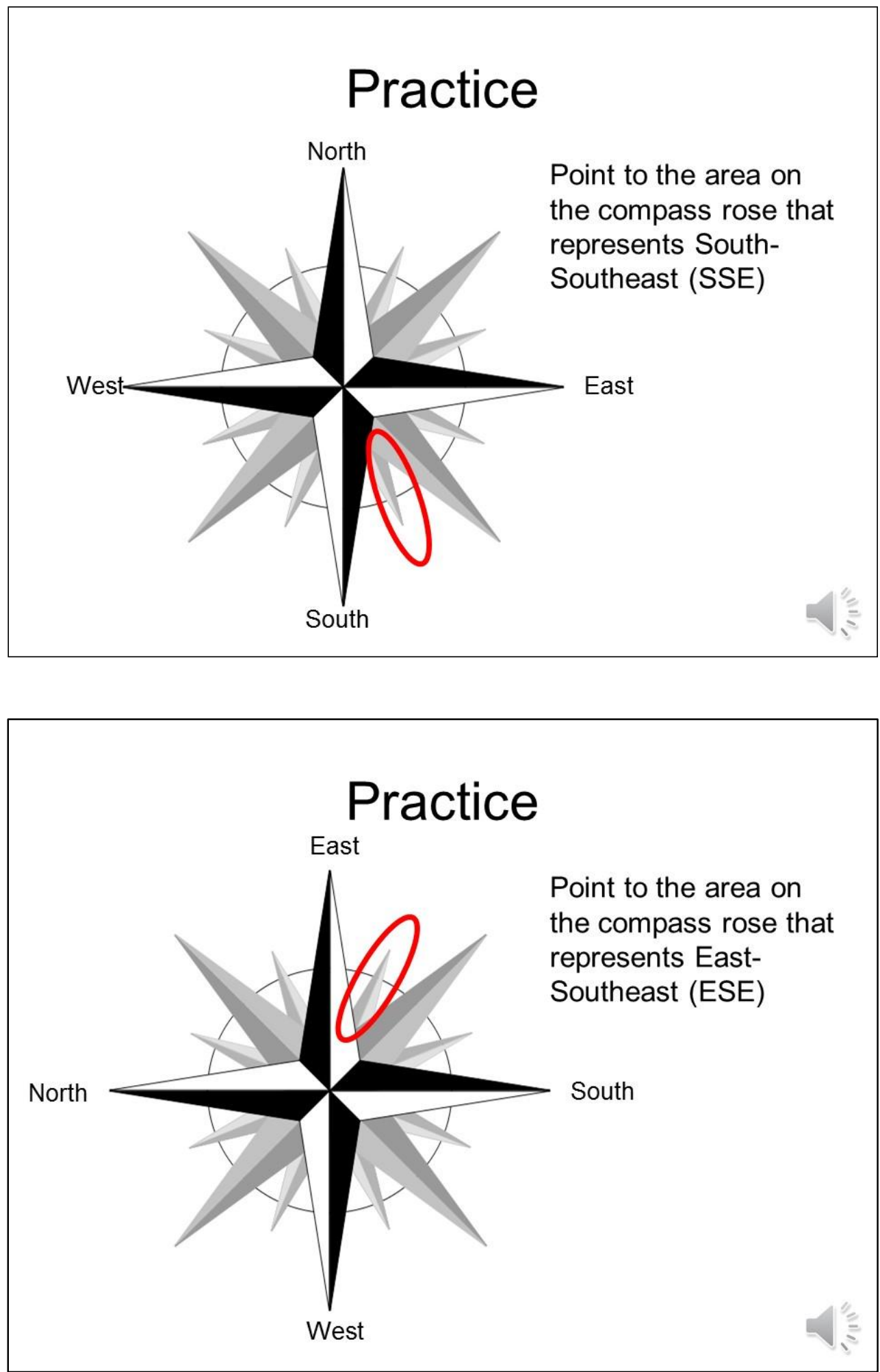


\section{Clock face directions}

- The robot may also provide extra information to the traveling updates.

- This information presents the direction the robot is traveling in terms of a clock face.

- Clock face directions are a form of relative directions, which are independent of cardinal direction (e.g., North, South, East, West).

- No matter the orientation cardinal directions, the clock face remains the same.

\section{Clock face}

- Imagine that you are in the center of a clock face.

North

- Straight ahead is 12 o'clock.

$-6=$ behind you

$-3=$ to your right

- $9=$ to your left

- Independent of cardinal directions.

- Aid in interpreting map orientation when North is not "straight ahead".

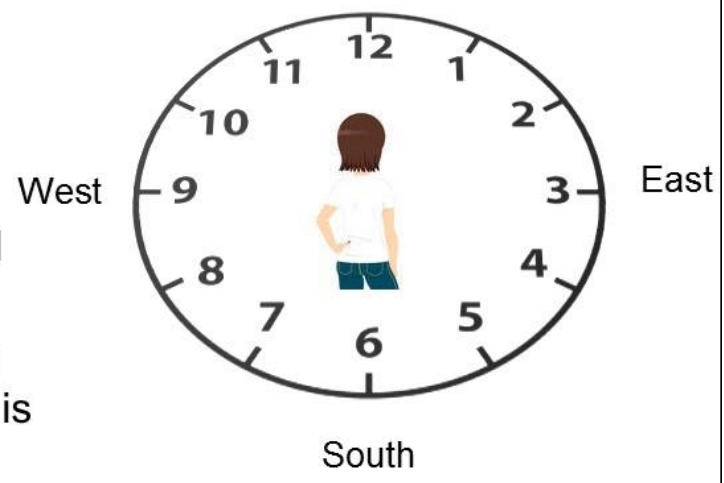




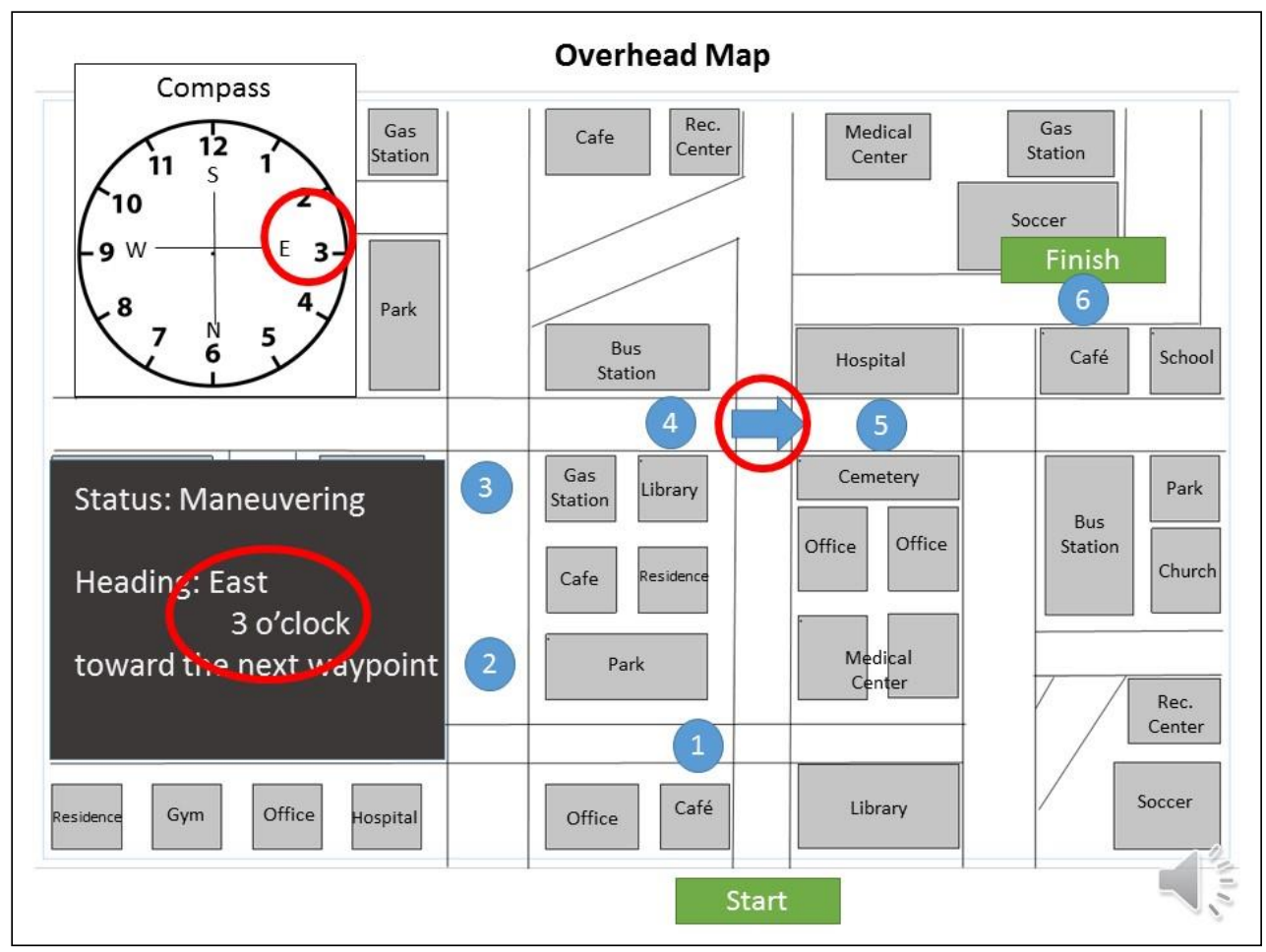

\section{Sensitive areas in the urban environment}




\section{Sensitive areas}

- For this operation, there are buildings in the environment that have been deemed sensitive and unnecessary for the surveillance and reconnaissance mission.

- As such, areas surrounding these buildings should be avoided by the robot while it travels through the environment.

- These buildings are:

- Medical centers

- Recreation (Rec.) centers

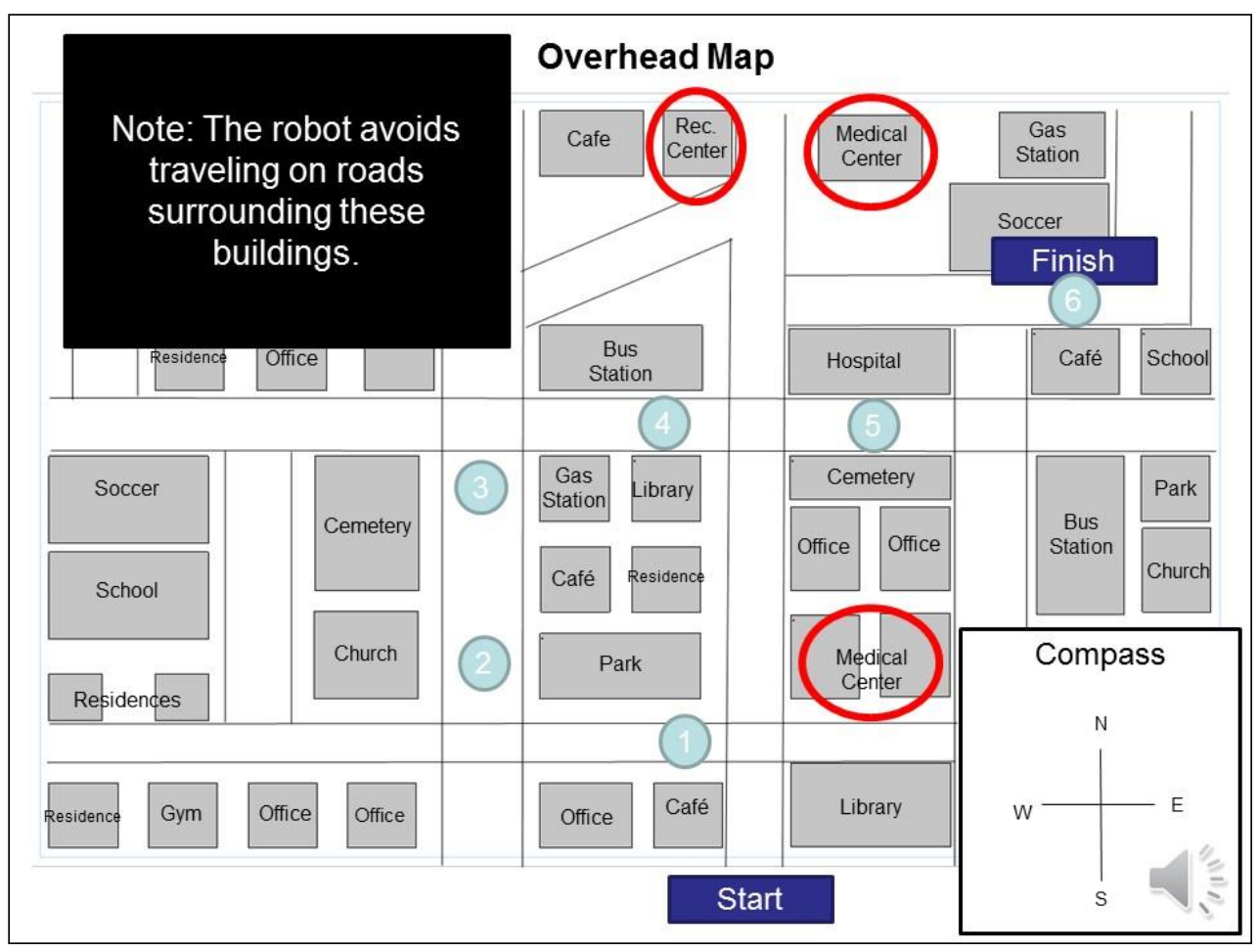




\section{Rerouting the robot: Avoiding sensitive buildings}

\section{Rerouting the robot}

- Because the robot is still in development and testing phases, there is a possibility that the robot may veer from its planned waypoint path through the environment.

- Periodically, you will be asked if the robot has veered from its path and needs to be re-routed through the environment.

- If the robot needs to be re-routed, you will be asked to provide feedback concerning the route the robot should take.

- Please note: the robot will use your feedback to correct its route in future paths through the environment. The robot will not alter its path after receiving feedback. 


\section{Rerouting the robot}

- Providing feedback when the robot has veered from its waypoint path entails drawing a path to the robot's next planned waypoint that:

- The shortest distance to the next waypoint

- Avoids traveling on roads that surround sensitive buildings.

- The following slides contain an example.

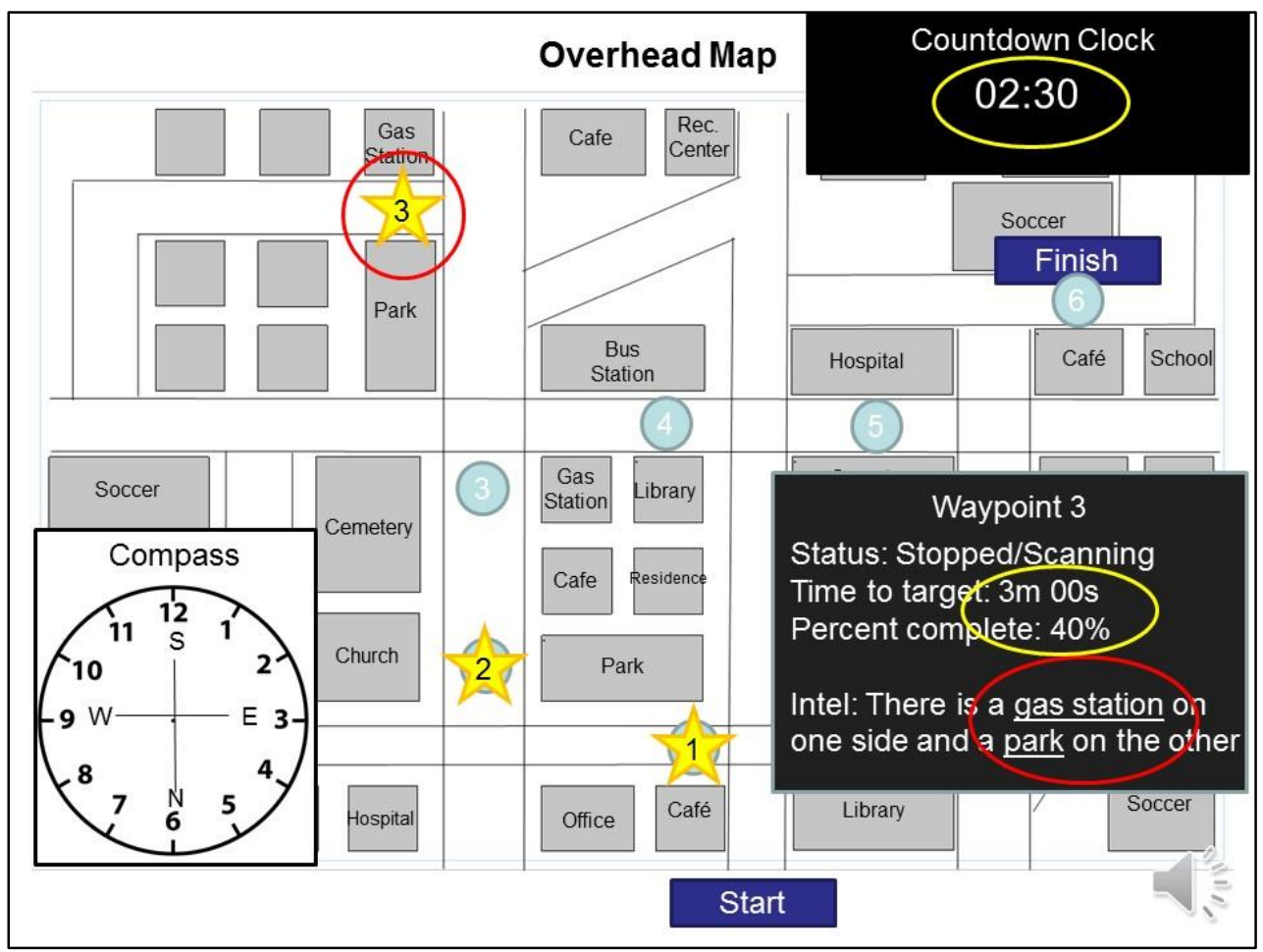




\section{Re-routing the robot}

- You will be asked to draw a route that brings the robot back to its planned waypoint path via a route that is:

- The shortest path

AND

- Avoids traveling on roads that surround rec. centers and medical centers

\section{Drawing routes}

- You will draw the routes by using materials provided in the 3-ring binder located on the table in front of you.

- Inside the binder you will see:

- Maps labeled with page numbers.

- A marker you will use for drawing.

If any of these materials are missing, please let the experimenter know.

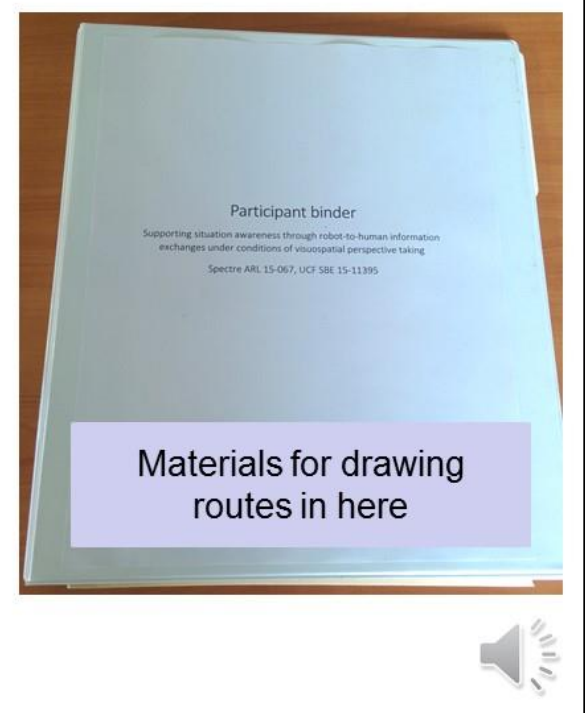



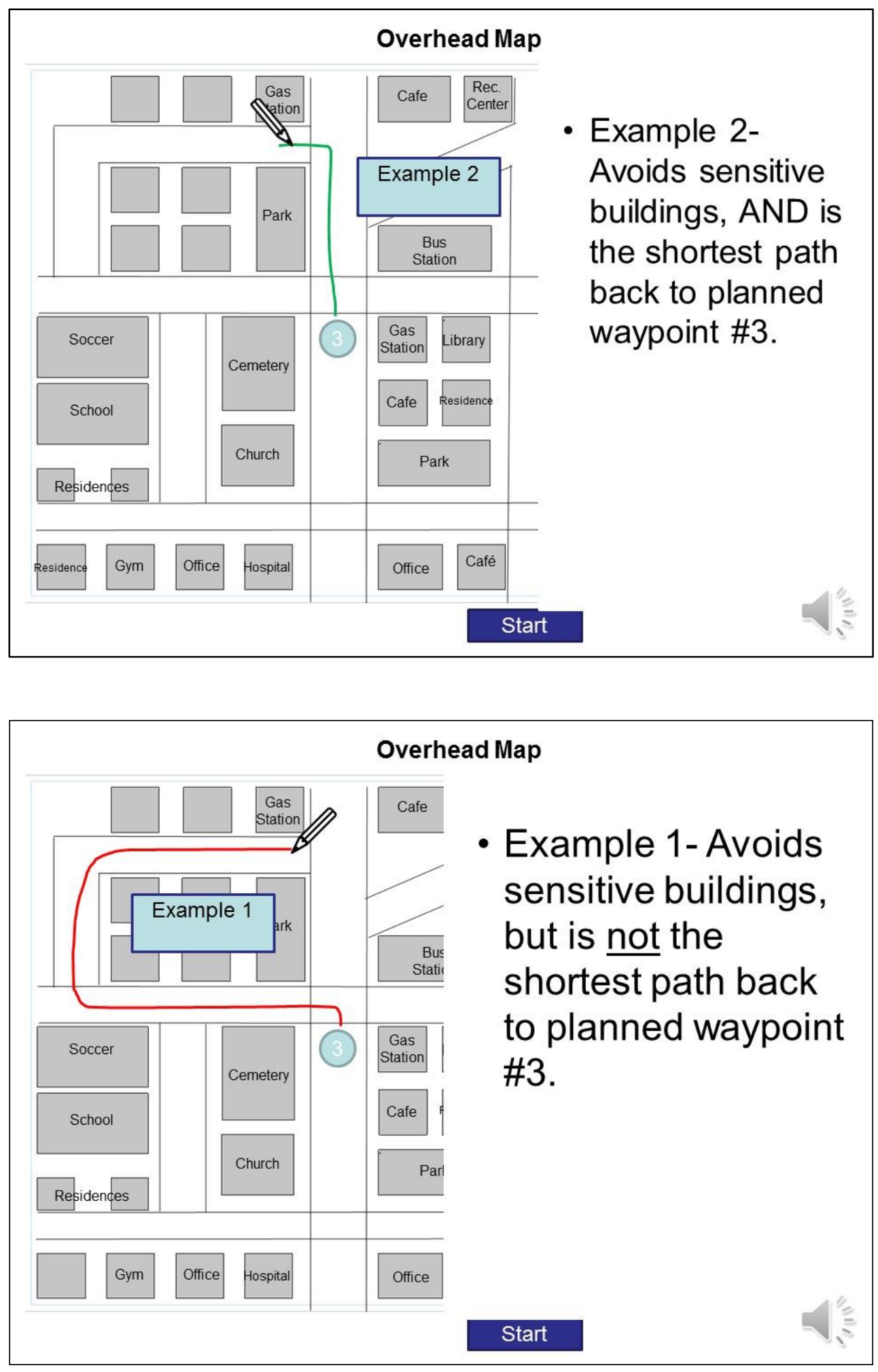


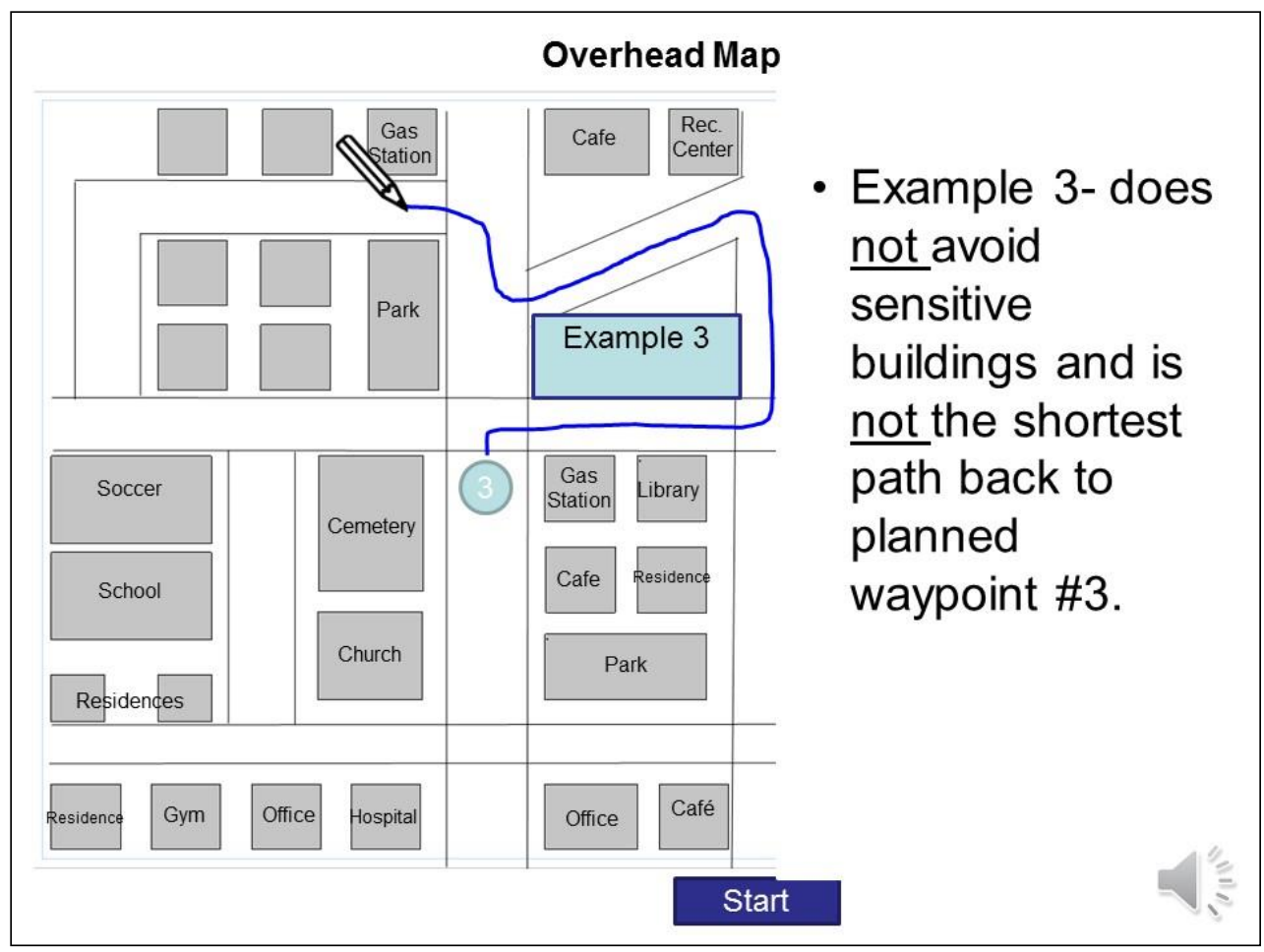

\section{Rerouting the robot}

- The drawing in example 2 is the best option for re-routing the robot back to its planned waypoint path.

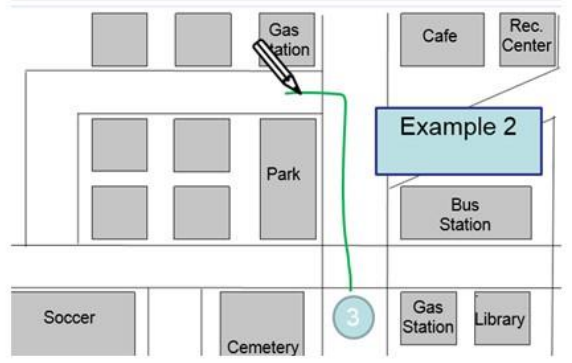




\section{STOP HERE}

- You will now be given an opportunity to practice rerouting the robot.

- If you have questions, please let the experimenter know.

\section{Answering questions}




\section{Answering questions}

Several times throughout each mission you will be asked to provide information concerning:

- Where the robot is located.

- Buildings located around the robot.

- The direction the robot is traveling in or will travel in.

- The remaining time left to complete the planned waypoint path.

- Whether the robot needs to be re-routed.

Please provide as much detailed information as you can to answer these questions and avoid guessing, as your answers will help us to refine the capabilities of the final version of the robot.

If you are unsure of an answer, please respond with "unsure" or "I don't know".

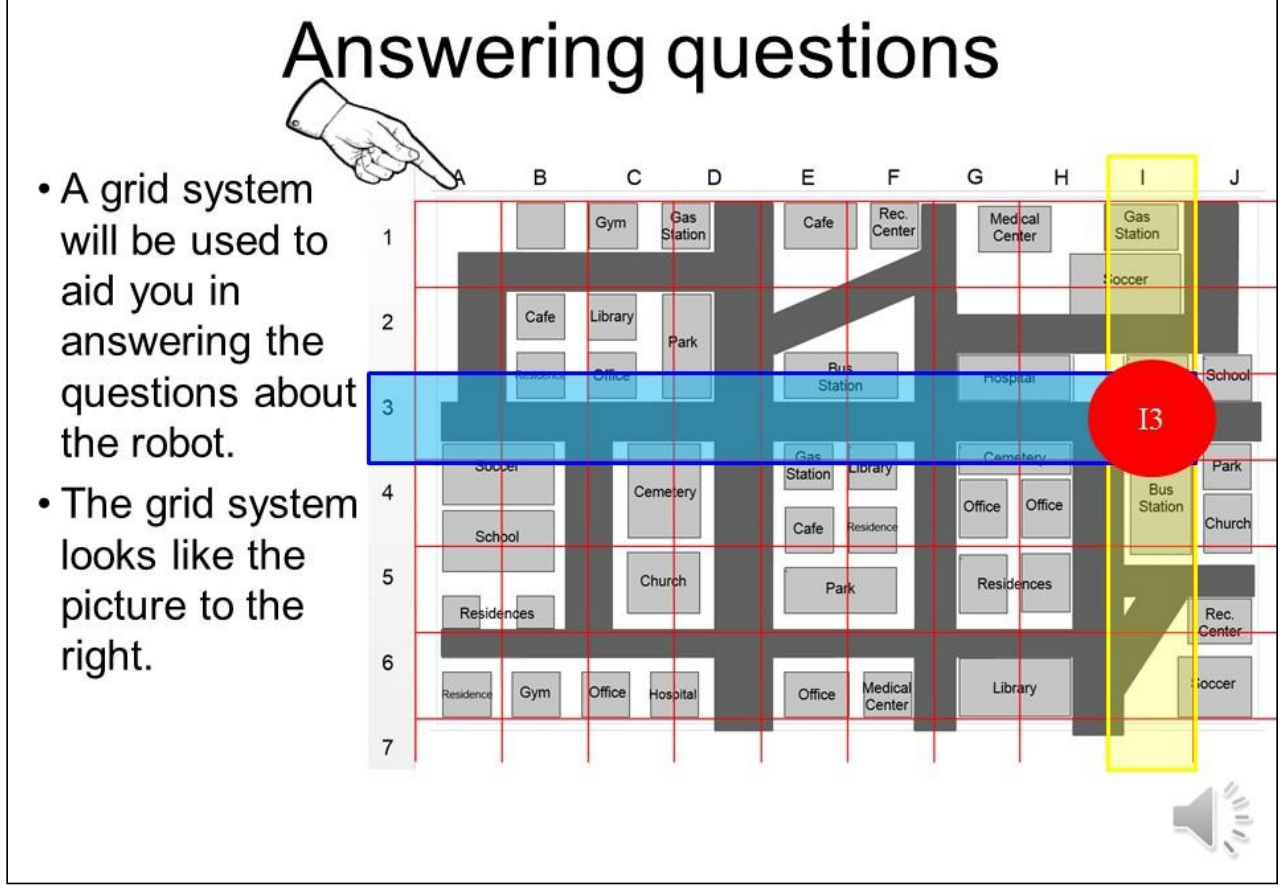




\section{NASA-TLX cont.}

- The NASA-TLX will consist of a visual prompt that will be automatically triggered several times throughout the study.

-When you see the prompt, you will respond with your answers.

- Answer the questionnaire based on your experiences since the last questionnaire.

\section{NASA-TLX questionnaire}

- The NASA-TLX measures 6 subscales of workload, each on a 100 point scale. The higher the number the higher the workload measure.

- The following subscales make up the NASA-TLX:

- Mental Demand:

- How much mental and perceptual activity was required (e.g., thinking, looking, searching, etc.)? Was the task easy or demanding, simple or complex, exacting or forgiving?

- Physical Demand:

- How much physical activity was required (e.g., pushing, pulling, turning, controlling, activating, etc.)? Was the task easy or demanding, slow or brisk, slack or strenuous, restful or laborious?

- Temporal Demand:

- How much time pressure did you feel due to the rate or pace at which the task or task elements occurred? Was the pace slow and leisurely or rapid and frantic?

- Effort:

- How hard did you have to work (mentally and physically) to accomplish your level of performance?

- Frustration:

- How insecure, discouraged, irritated, stressed and annoyed versus secure, gratified, content, relaxed and complacent did you feel during the task?

- Performance:

- How successful do you think you were in accomplishing the goals of the task set by the experimenter (or yourself)? How satisfied were you with your performance in accomplishing these goals?

- IMPORTANT NOTE: Each subscale is measured from low (left) to high (right) except the performance subscale which measures from good (left) to poor (right). Please keep this in mind when filling out the NASA-TLX. 

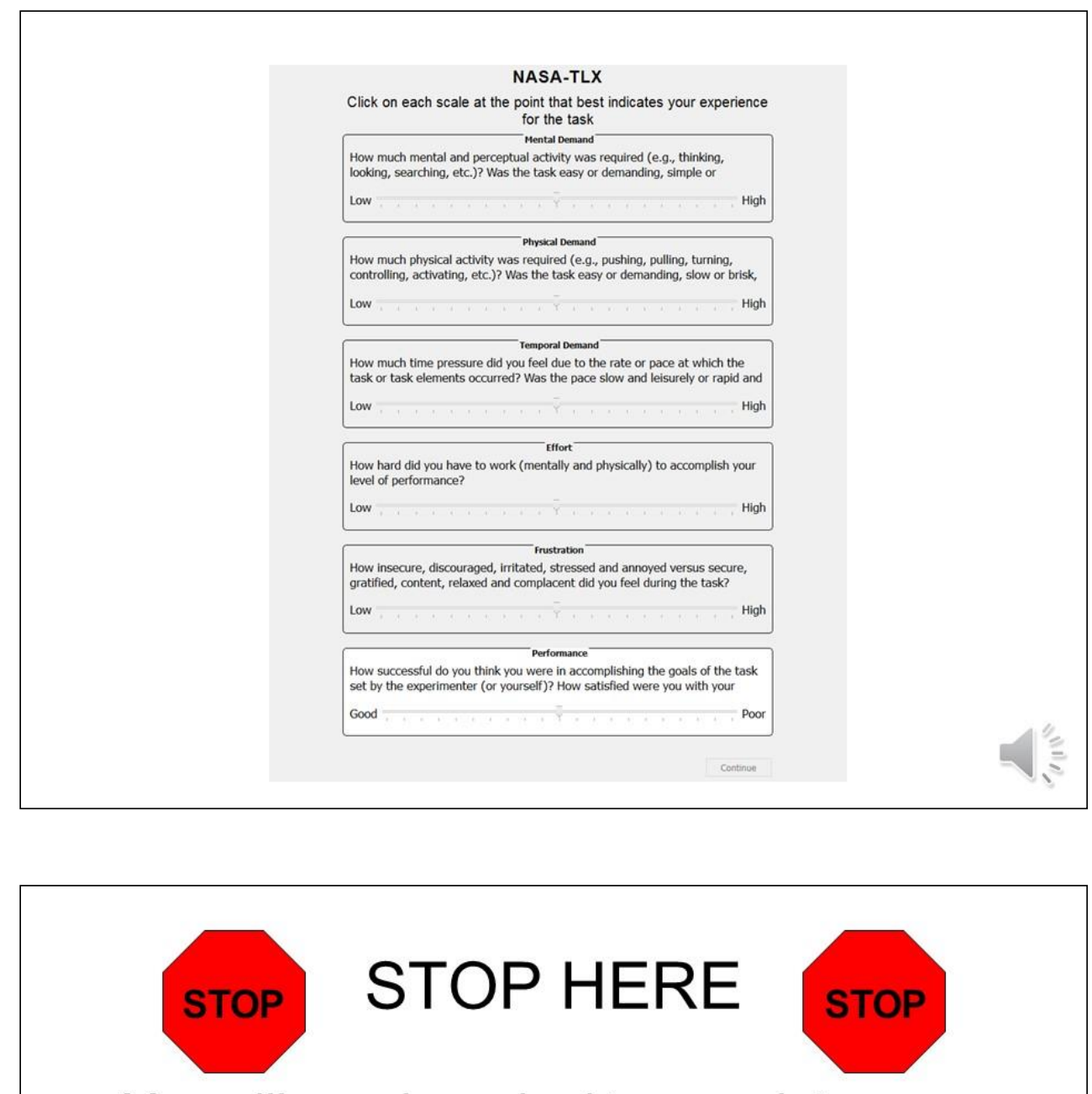

- You will now be asked to complete a series of practice missions in which you will practice receiving updates from the robot, answering questions, and rerouting the robot.

- If, after the following training, you feel like you need more practice, please let the experimenter know.

- After completing these missions, your training will be complete 


\section{APPENDIX M: PARTICIPANT TRAINING SLIDE SCRIPT}




\section{Training Slides Narration Transcription}

1. Welcome to today's experiment. The following slides will provide you with the information you need to complete today's study. It is important to remember that if at any time you need assistance or have questions, to please ask the experimenter who is facilitating today's study. Let's get started.

2. The Department of Defense is testing new deployable technology to be used by dismounted Soldiers during intelligence, surveillance, and reconnaissance, also known as ISR operations. Some of these new technologies include robots that are capable of full autonomy. Meaning they can be commanded to carry out a mission task without having to be within proximity of the rest of the squad or need direct continuous supervision.

3. However, this robotic technology is still in a testing phase. At this point, there are technological and design issues that need to be addressed and we need your help to resolve these problems.

The robot is fully equipped with a host of sensors that allow it to capture data from the environment in order to navigate over various terrain, maneuver around various obstructions, and identify objects and buildings, but the robot needs assistance in order to correctly navigate through these areas of deployment.

The robot that will be used in this study will be simulated by computerized software that emulates the robot's sensing equipment.

4. Your role is to work together with the simulated robot on a reconnaissance and surveillance mission by completing the following main objectives.

1. Provide surveillance by monitoring changes to an area of interest in the environment.

2. Help the robot to move through an urban environment as quickly as possible while simultaneously avoiding sensitive areas in the environment.

3 . Help the robot to learn alternate routes through the environment, in the event that it veers off course.

4. Work to complete the mission in a timely manner.

5. For this study, you will be asked to:

○ Play the role of a Soldier in a simulated Soldier-robot team. 
- Provide surveillance of an area of interest and make note of changes to that area.

- Use updates from the robot to keep track of the robot's movement and answer questions regarding the robot's movement and location.

- Provide re-routing information that helps the robot to avoid sensitive areas, in the event that the robot veers off course.

- Pay attention to how quickly the robot is completing its objectives.

6. In addition, the robot will:

- Provide reconnaissance by navigating a waypoint path through the environment.

- Periodically share updates with you regarding the buildings located at each waypoint, as well as other status information concerning its navigation.

- The following slide will provide an example of a robot's planned waypoint path through the environment.

7. In this study, you will be asked to complete 4 missions with the robot. For each mission, an overhead map of the mission environment, like the one depicted on this slide, will be provided to you. Please note, that the numbered circles denote the robot's planned waypoint stops along its path through the environment. The robot's plan to start and end points are also depicted on this map. And, the distance between each waypoint should be approximately equal and the robot will travel between waypoints at a constant rate. Again, for each mission, a map like this will be provided to you.

8. The slides in this section will provide information concerning your task to provide surveillance to an area of interest. If you have any questions during this time, please feel free to ask the experimenter.

9. Throughout the course of the study, you will see an overhead view of an area of interest. You will be asked to monitor changes to icons displayed in this view. There are three types of changes the icons can make. They are: appear, disappear, and move. When you observe a change, you will be asked to indicate whether the icons appeared, disappeared, or moved by clicking on the buttons displayed on the screen.

10. For the "appear" changes, new icons will be added to the array of icons displayed on the screen.

- Indicate that icons have appeared by using the mouse cursor to click the "Appeared" button. 
11. For the "disappeared" changes, existing icons will leave the screen.

- Indicate that icons have disappeared by using the mouse cursor to click the "Disappeared" button.

12. For the "movement" changes, existing icons will move from one position to another.

- Indicate that icons have moved by using the mouse cursor to click the "Movement" button.

13. Please stop here.

- You will now practice the surveillance task by monitoring changes to the area of interest.

- If, after the following training, you feel like you need more practice, please let the experimenter know.

14. The next section will provide you with information concerning the countdown clock and the team's objective to finish the mission in a timely manner.

15. Throughout the course of each mission, a countdown clock will be present.

- Each mission should take approximately 5 minutes to complete and the countdown will begin at the start of each mission.

- The robot is expected to reach its final waypoint by the time the countdown clock reaches zero.

- You will be asked to keep an eye on this countdown to determine whether or not the robot is going to complete its waypoint path before the clock runs out.

16. The slides in this section will cover the reconnaissance updates from the robot, as well as how to interpret the information given in these updates. Again, if you have questions, please feel free to ask the experimenter.

17. Throughout the course of each mission, the robot will periodically share intelligence and reconnaissance information with you concerning:

- The status of the robot's waypoint navigation through the environment, as well as, Intelligence concerning the buildings located around each waypoint stop.

- The robot will provide two types of updates. The following slides will provide examples of each type. They are:

i. Waypoint updates and Traveling updates 
18. Waypoint updates:

- When the robot reaches a waypoint, it will stop, scan the environment, and then provide information concerning the buildings located within its immediate area.

- The robot will also provide other status information at this time.

- Waypoint updates will be given to you each time the robot stops at a waypoint along its path.

- The robot will always provide an update each time it stops.

19. There are several pieces of information that will be provided to you in the waypoint update. This slide contains an example of a waypoint update.

$\circ$ Note, the first piece of information is the robot's status as stopped and scanning the environment. The robot also provides the estimated time it thinks it will take to complete the remainder of its route.

- The robot also provides an approximation of the percentage of the route that it thinks it has completed. In this case, the robot is reporting that it thinks it has completed approximately $16 \%$ of the route through the environment.

- Finally, the robot will provide intelligence concerning the buildings that are located in its immediate area. In this case, the robot has reported that there is a church on one side of the robot and a park on the other.

20. Here is the waypoint update in relationship to the overhead map of the environment and the robot's planned path through the environment.

- Based on this waypoint update, we can decipher that the robot is located at the area circled in red on this map. That is because the area circled in red has a church on one side of the street and a park on the other.

$\circ$ In addition, this area seems to be approximately $16 \%$ of the way through the robot's planned waypoint path through the environment.

- Finally, the estimated time to reach the end of the robot's path matches the time remaining on the countdown clock.

- This indicates that the robot is on track to complete its waypoint path by the time the clock reaches zero. Based on this information, we can conclude that the robot is currently located at waypoint number two.

21. Traveling updates:

o Periodically, the robot will also share information with you while it is traveling from one waypoint to another.

- Traveling updates will provide you with information concerning the direction the robot is traveling.

- Because the robot is still in testing phases, the robot will provide traveling updates in some instances and not in others. 
- In addition, the robot may be able to provide extra traveling information in some instances and not others. We'll cover what this extra information is in other slides.

22. Here is an example of a traveling update. There are a couple of pieces of information that will be provided to you in the traveling update.

$\circ$ Note, the first piece of information is the robot's status as maneuvering between waypoints.

- In addition, the robot will provide the direction that it's traveling between waypoints. In this instance, the robot is traveling north from one waypoint to another.

23. Here is the traveling update in relationship to the overhead map of the environment and the robot's planned path through the environment.

○ The last time we heard from the robot, we deciphered that the robot was at waypoint number two. We know that the robot is traveling north towards the next waypoint.

- Using this information and the compass rose located on the map, we can conclude that the robot is indeed traveling towards waypoint number three.

- That is because waypoint number three is towards the north of waypoint number two. We will talk more about the compass rose in later slides.

24. The slides contained in this section will cover the compass rose that will be depicted on the overhead maps that will be provided to you. The slides will also cover how to interpret and read various compass roses. If you have questions while reviewing this information, please feel free to ask the experimenter.

25. A compass rose will be depicted on the overhead map that shows the robot's planned waypoint path through the environment.

- A compass rose is a figure on a map or chart used to display the orientation of cardinal directions. That is, North, South, East, and West relative to the map or chart.

- The compass rose can also display intermediate directions as well as combination points.

26. Here is an example of a compass that displays cardinal directions.

- Cardinal directions are North, South, East, and West. Note that the cardinal directions are depicted at right angles on the compass rose.

27. Here is an example of a compass that displays cardinal and intermediate directions. 
- Intermediate directions lie between the cardinal directions on the compass rose at 45 degree angles.

○ They are: Northeast, Northwest, Southeast, and Southwest.

28. Finally, here is an example of a compass that displays cardinal, intermediate, and combination point directions.

- Combination points lie between intermediate and cardinal directions and are a combination of the two.

- These fall at approximately 22 degree angles on the compass.

○ The combination point's directions, going in clockwise order, are: Northnortheast, East-Northeast, East-southeast, South-southeast, Southsouthwest, West-southwest, West-northwest, and North-northwest.

29. Combination point directions can be a little confusing because there are so many.

o Just remember that the name and convention starts with the cardinal direction listed first, and then the intermediate direction listed second.

$\circ$ The combination point lies between these two directions.

$\circ$ In this instance, the combination point, North-Northeast is located between north and northeast.

30. Combination points are read this way even if the orientation of the map has changed. In this example, the orientation of the map is such that north is now located to the left-hand side. The combination point North-Northeast is still located between North and Northeast. The same is true for West-southwest. We'll do a couple of practice exercises in the remaining slides.

31. Let's do some practice exercises. Please point to the area on the compass rose that represents South-Southeast.

- Good! Circled in red is the correct answer because the combination point, south-southeast, lies between the cardinal and intermediate directions of south and southeast on the compass rose.

32. Let's try another. Please point to the area on the compass rose that represents East-Southeast. Make sure to note that the orientation of the compass rose has changed.

- Good! Circled in red is the correct answer because the combination point, East-southeast, lies between the cardinal and intermediate directions of east and southeast on the compass rose. If you have more questions about this, please make sure to ask your experimenter. 
33. The robot may also provide extra information to the traveling updates.

$\circ$ This information presents the direction the robot is traveling in terms of a clock face.

- Clock face directions are a form of relative directions, which are independent of cardinal directions like North, South, East, and West.

o This means that no matter the orientation of cardinal directions, the clock face remains the same. We'll see an example of how to understand clock face directions in the following slide.

34. To understand the clock face directions, imagine that you are standing in the center of a clock face.

- Straight ahead of you is 12 o'clock. This means that 6 o'clock is directly behind you, 3 o'clock is to your right, and 9 o'clock is to your left.

- The great thing about clock face directions is that they are independent of cardinal directions. 12 o'clock is always directly ahead of you, no matter the orientation of the cardinal directions.

- In this instance, North is now located to your right, but 12 o'clock remains the same. This is helpful in instances in which the orientation of North does not always correspond to straight ahead on the map. We'll do an example in just a second.

35. Here is an example of a traveling update with the extra clock face direction information.

○ In this example, the robot is traveling towards waypoint number 5 in the direction of 3 o'clock, which corresponds to the cardinal direction east.

- The clock face information, along with the orientation of the cardinal directions, is depicted in the compass rose located in the upper corner of the map.

36. The following section will cover information concerning sensitive areas of the environment. These include buildings that the robot should avoid when traveling through the environment. If after reviewing these slides you have questions, please feel free to ask the experimenter.

37. For this operation, there are buildings in the environment that have been deemed sensitive and unnecessary for the surveillance and reconnaissance mission.

$\circ$ As such, areas surrounding these buildings should be avoided by the robot while it travels through the environment.

○ These buildings are: Medical centers and Recreation (Rec.) centers 
38. Please note the location of medical and rec. centers on this map.

- You will note that the robot's planned path tries to avoid traveling on roads that surround these types of buildings.

$\circ \quad$ The next section will provide you with more information concerning rerouting the robot and avoiding these types of buildings.

39. The information contained in the following slides concerns rerouting the robot in the event that the robot veers from its planned waypoint path through the environment. When rerouting the robot, it is important to avoid sensitive buildings. The following slides will be dedicated to explaining how to reroute the robot and avoid sensitive buildings in more detail.

40. Because the robot is still in development and testing phases, there is a possibility that the robot may veer from its planned waypoint path through the environment.

- Periodically, you will be asked if the robot has veered from its path and needs to be re-routed through the environment.

o If the robot needs to be re-routed, you will be asked to provide feedback concerning the route the robot should take.

- Please note: Due to signal strength issues, the robot will use your feedback to correct its route in future paths through the environment. The robot will not alter its path after receiving your feedback.

41. Slide 41: Providing feedback when the robot has veered from its waypoint path entails drawing a path to the robot's next waypoint that:

- Is the shortest distance to the next waypoint AND avoids traveling on roads that surround sensitive buildings.

- The following slides will contain an example of how to choose a path to the next waypoint.

42. Let's look at an example. We'll use an overhead map to conceptually represent the robot's planned waypoint path and its actual waypoint path through the environment.

- On this map, you can see the robot's planned waypoint path. Planned waypoint paths are denoted by circles. Stars represent the actual waypoints where the robot has already stopped. That is, this is the path that the robot has actually taken up until this point.

- Let's see what the robot's next waypoint update has to tell us.

- Take a moment to look at all of the information that is contained in this slide.

- You can see that the robot has veered off of the planned waypoint path at waypoint three. 
- We know this because the waypoint update has indicated that the robot is located between a gas station and a park and not between a gas station and a cemetery, which is where it would be if it was actually at planned waypoint three.

- The robot will need to be rerouted back to the original planned waypoint three.

43. When rerouting the robot, you will be asked to draw a route that brings the robot back to its planned waypoint path via a route that is:

- The shortest path and

- Avoids traveling on roads that surround recreation and medical centers.

44. You will draw the routes using materials provided in the 3-ring binder located on the table in front of you.

- Inside the binder you will see:

i. Maps labeled with page numbers

ii. A marker you will use for drawing

- If any of these materials are missing, please let the experimenter know.

- Let's look next at some options for rerouting the robot in our next example.

$\circ$ When rerouting, you will be asked to draw the route that brings the robot back to its planned waypoint path via a route that is:

i. the shortest path to the waypoint AND avoids traveling on roads that surround sensitive buildings like rec. centers and medical centers.

- Let's look next at some examples of drawing a new route for the robot in the next few slides.

45. (Start animation on slide start). The route drawn in example 1 avoids roads that surround sensitive buildings, but is not the shortest path back to planned waypoint 3.

46. (Start animation on slide start). The route drawn in example 2 avoids roads that surround sensitive buildings and is the shortest path back to planned waypoint 3 .

47. (Start animation on slide start). The drawing in example 3 does not avoid traveling on roads that surround sensitive buildings and is also NOT the shortest path back to planned waypoint 3 .

48. As such the drawing in example 2 is the best option for re-routing the robot back to its planned waypoint path. We'll do a practice exercise next. 
49. Please stop here. You will now be given an opportunity to practice rerouting the robot. If you have questions, please let the experimenter know.

50. The following slides will contain information regarding how to answer questions during the course of the study. If you have questions, please let the experimenter know.

51. Several times throughout each mission, you will be asked to provide information concerning:

- Where the robot is located

- Buildings located around the robot

- The direction the robot is traveling in or will travel in

o The remaining time left to complete the planned waypoint path

- Whether or not the robot needs to be rerouted.

- Please provide as much detailed information as you can to answer these questions and avoid guessing as your answers will help us to refine the capabilities of the final version of the robot

- If you are unsure of an answer, please respond with "unsure" of "I don't know"

52. A grid system will be used to help you answer questions. For instance, when responding to the question, "Where is the robot?" you may want to indicate that the robot it located in the are circled in yellow on the map. To respond the question, "Where is the robot?" you will use a combination of the letters and numbers located on the top and left side of the grid. To read the grid you first read *across* the top columns of letters **/and then ${ }^{*}$ down $*$ the left rows of **numbers. In this example, the robot is located at Grid Block *"eye three"*. Because the location of the robot on the grid falls in column I and row 3.

53. The NASA-TLX will consist of a visual prompt that will be automatically triggered several times throughout the study. When you see the prompt, you will respond with your answers. Answer the questionnaire based on your experiences since the last questionnaire.

54. The NASA-TLX measures 6 subscales of workload, each on a 100 point scale. The higher the number the higher the workload measure.

- The following subscales make up the NASA-TLX:

- Mental Demand:

i. How much mental and perceptual activity was required (e.g., thinking, looking, searching, etc.)? Was the task easy or demanding, simple or complex, exacting or forgiving? 
- Physical Demand:

i. How much physical activity was required (e.g., pushing, pulling, turning, controlling, activating, etc.)? Was the task easy or demanding, slow or brisk, slack or strenuous, restful or laborious?

- Temporal Demand:

i. How much time pressure did you feel due to the rate or pace at which the task or task elements occurred? Was the pace slow and leisurely or rapid and frantic?

- Effort:

i. How hard did you have to work (mentally and physically) to accomplish your level of performance?

○ Frustration:

i. How insecure, discouraged, irritated, stressed and annoyed versus secure, gratified, content, relaxed and complacent did you feel during the task?

- Performance:

i. How successful do you think you were in accomplishing the goals of the task set by the experimenter (or yourself)? How satisfied were you with your performance in accomplishing these goals?

ii. IMPORTANT NOTE: Each subscale is measured from low (left) to high (right) except the performance subscale which measures from good (left) to poor (right). Please keep this in mind when filling out the NASA-TLX.

55. Here is an additional look at the NASA-TLX. Again, please note that responses go from low on the right to high on the left except for the performance scale which goes from good on the right to poor on the left.

56. Please stop here. You will now be asked to completed a series of practice missions in which you will practice receiving updates from the robot, answering questions, and rerouting the robot.

- If after the following training, you feel like you need more practice, please let the experimenter know.

- After completing these missions, your training will be complete. 


\section{LIST OF REFERENCES}

Abich, IV, J., Barber, D., Talone, A.B., Phillips, E., Jentsch, F., Pettitt, R., \& Elliott, L. (2016). Soldier-robot team communications: An investigation of exogenous orienting visual display cues and robot reporting preferences (Contract No. W911NF-10-20016). Army Research Laboratory.

Abich IV, J., Reinerman-Jones, L., \& Taylor, G. (2011). Establishing workload manipulations utilizing a simulated environment. In Virtual, Augmented and Mixed Reality. Systems and Applications (pp. 211-220). Springer Berlin Heidelberg.

Army Research Laboratory (2012). Robotics Collaborative Technology Alliance (RCTA) FY 2012 annual program plan. Retrieved from http://www.arl.army.mil/www/pages/392/RCTA_FY12_APP.pdf

Army, U. S. (2008). The Warrior Ethos and Soldier Combat Skills: Field Manual 321.75, Washington, DC: Headquarters, Department of the Army.

Army, U.S (2012). Doctrine and Training Publications: Offense and Defense ADRP 3 90, Washington, DC: Headquarters, Department of the Army.

Babyak, M.A. (2009). Understanding confounding and mediation. Evidence Based Mental Health, 12, 68-71. doi: 10.1136/ebmh.12.3.68

Baddeley, A. (1992). Working memory. Science, 255(5044), 556-559. 
Barber, D., Abich IV, J., Phillips, E., Talone, A.B., \& Jentsch, F. (2015). Field assessment of multimodal communication for dismounted human-robot teams. Proceedings of the Human Factors and Ergonomics Society Annual Meeting, 59(1), 921-925. doi: $10.1177 / 1541931215591280$

Barber, D., Wohleber, R.W., Parchment, A., Jentsch, F., \& Elliot, L. (2014).Development of a Squad Level Vocabulary for Human-Robot Interaction. In Virtual, Augmented and Mixed Reality. Designing and Developing Virtual and Augmented Environments, $139-148$.

Barber, D.J., Leontyev, S., Sun, B., Davis, L., Chen, J.Y.C., \& Nicholson, D. (2008). The Mixed-Initiative Experimental (MIX) Testbed for collaborative human robot interactions. Proceedings of the 2008 International Symposium on Collaborative Technologies and Systems (CTS), January 29, 483-489.

Brown, J., Gray, J. P., Blanco, C., Juneja, A., Alberts, J., \& Reinerman, L. (2011). Handsfree, Heads-up Control System for Unmanned Ground Vehicles (No. TARDEC22037). Retrieved from http://www.dtic.mil/dtic/tr/fulltext/u2/a547423.pdf

Burke, J. L., \& Murphy, R. R. (2004, September). Human-robot interaction in USAR technical search: Two heads are better than one. In Robot and Human Interactive Communication, 2004. ROMAN 2004. 13th IEEE International Workshop on (pp. 307-312). IEEE. 
Burke, J. L., Murphy, R. R., Coovert, M. D., \& Riddle, D. L. (2004). Moonlight in Miami: Field study of human-robot interaction in the context of an urban search and rescue disaster response training exercise. Human-Computer Interaction, 19(1-2), 85116.

Carretta, T.R., Perry, D.C., \& Ree, M.J. (1996). Prediction of situational awareness in F15 pilots. The International Journal of Aviation Psychology, 6(1), 21-41.

Carlson, L.A. (1999). Selecting a reference frame. Spatial Cognition and Computation, 1(4), 365-379.

Casper, J., \& Murphy, R. R. (2003). Human-robot interactions during the robot-assisted urban search and rescue response at the World Trade Center. Systems, Man, and Cybernetics, Part B: Cybernetics, IEEE Transactions on, 33(3), 367-385. doi: 10.1109/TSMCB.2003.811794

Chen, J. Y. C. (2011). Individual differences in human - robot interaction in a military multitasking environment. Journal of Cognitive Engineering and Decision Making, 5 (1), 83-105. doi: 10.1177/1555343411399070

Chen, J. Y., Barnes, M. J., \& Qu, Z. (2010). RoboLeader: An agent for supervisory control of multiple robots. In Proceedings of the 5th ACM/IEEE international conference on Human-robot interaction (pp. 81-82). IEEE Press. 
Chen, J. Y. C., Haas, E. C., \& Barnes, M. J. (2007). Human performance issues and user interface design for teleoperated robots. Systems, Man, and Cybernetics, Part C: Applications and Reviews, IEEE Transactions on, 37(6), 1231-1245.

Cinaz, B., Bert, A., La Marca R., Tröster, G. (2013). Monitoring mental workload levels during an everyday life office-work scenario. Personal and Ubiquitous computing, 17(2), 229-239.

Corsi, P. M. (1972). Human memory and the medial temporal region of the brain. Dissertation Abstracts International, 34 (02), 891B. (University Microfilms No. AAI05-77717). -

Department of Defense, U.S. (2009). The Dictionary of Military Terms, Washington, D.C.

Department of Defense, U.S. (2013) 2013 Demographics: Profile of the Military Community, Washington, D.C.

Drury, J. L., Keyes, B., \& Yanco, H. A. (2007, March). LASSOing HRI: Analyzing situation awareness in map-centric and video-centric interfaces. In Human-Robot Interaction (HRI), 2007 2nd ACM/IEEE International Conference on (pp. 279-286). IEEE. 
Drury, J.L., Richer, J. Rackliffe, N., \& Goodrich, M.A. (2006). Comparing situation awareness for two unmanned aerial vehicle human interface approaches. Bedford, MA: MITRE Corporation.

Endsley, M. R. (1995a). Toward a theory of situation awareness in dynamic systems. Human Factors: The Journal of the Human Factors and Ergonomics Society, 37(1), $32-64$.

Endsley, M.R. (1995b). Measurement of situation awareness in dynamic systems. Human Factors: The Journal of the Human Factors and Ergonomics Society, 37(1), 65-84.

Endsley, M.R. (1988). Design and Evaluation for situation awareness enhancement, Proceedings of the Human Factors and Ergonomics Society Annual Meeting, 32, 97 101.

Endsley, M.R., \& Bolstad, C. A. (1994). Individual differences in pilot situation awareness. The International Journal of Aviation Psychology, 4 (3), 241-264.

Faul, F., Erdfelder, E., Lang, A.-G., \& Buchner, A. (2007). G*Power 3: A flexible statistical power analysis program for the social, behavioral, and biomedical sciences. Behavior Research Methods, 39, 175-191.

Fendrich, L. (2008, July 30). Is the analog clock out of time? Chronicle of Higher Education. Retrieved from http://chronicle.com/blogs/brainstorm/is-the-analog-clockout-of-time/6171 
Fincannon, T.D. (2013). Visuo-spatial abilities in remote perception: A meta-analysis of empirical work. (Unpublished doctoral dissertation). University of Central Florida, Orlando, Florida.

Fioratou, E., Flin, R., Glavin, R., \& Patey, R. (2010). Beyond monitoring: Distributed situation awareness in the heart room. British Journal of Anaesthesia, 105 (1), 83-90.

Flavell, J.H. (1992). Perspectives on perspective taking. In H. Beilin \& P.B. Pufall (Eds.), Piaget's theory: Prospects and possibilities (pp. 107-140). Hillsdale, NJ: Lawrence Erlbaum Associates.

Fong, T., Kunz, C., Hiatt, L. M., Bugajska, M. (2006). The human-robot interaction operating system. In Proceedings of the $1^{\text {st }}$ AMC SIGCHI/SIGART Conference on Human-robot Interaction, 41-48.

Fong, T., Thorpe, C., \& Baur, C. (2003). Collaboration, Dialogue, Human-Robot Interaction. In R. Jarvis \& A. Zelinsky (Eds.), Robotics Research (Vol. 6, pp. 255266): Springer Berlin Heidelberg.

Freeman, S. (2009). Teachers say children are becoming so familiar with digital clocks, some do not know how to read traditional ones. Mass Live. Retrieved from http://www.masslive.com/news/index.ssf/2009/12/teachers_say_children_are_beco.ht $\underline{\mathrm{ml}}$ 
Groom, V., \& Nass, C. (2007). Can robots be teammates?: Benchmarks in human—robot teams. Interaction Studies, 8(3), 483-500.

Hart, S. G., \& Staveland, L. E. (1988). Development of NASA-TLX (Task Load Index): Results of empirical and theoretical research. Advances in psychology, 52, 139-183.

Hazlehurst, B., McMullen, C.K., \& Gorman, P. N. (2007). Distributed cognition in the heart room: How situation awareness arises from coordinated communications during cardiac surgery. Journal of Biomedical Informatics, 40 (2007), 539-551.

Hegarty, M. \& Waller, D. (2004). A dissociation between mental rotation and perspective-taking spatial abilities. Intelligence, 32 (2004), 175-191.

Horrey, W. J., Lesch, M. F., \& Garabet, A. (2009). Dissociation between driving performance and drivers' subjective estimates of performance and workload in dualtask conditions. Journal of safety research, 40(1), 7-12.

Jackel, L.D., Hackett, D., Krotkov, E., Perschbacher, M., Pippine, J., \& Sullivan, C. (2007). How DARPA structures its robotics programs to improve locomotion and navigation. Commun. ACM, 50(11), 55-59. doi: 10.1145/1297797.1297823

Johannesen, L. J., Cook, R. I., \& Woods, D. D. (1994). Cooperative communications in dynamic fault management. Proceedings of the Human Factors and Ergonomics Society Annual Meeting, 38(4), 225-229. doi:10.1177/154193129403800408 
Kelly, J.W., \& McNamara, T.P. (2010). Reference frames during the acquisition and development of spatial memories. Cognition, 116, 409-420.

Kozhevnikov, M. \& Hegarty, M. (2001). A dissociation between object-manipulation and perspective-taking spatial abilities. Memory \& Cognition, 29, 745-756.

MacMillan, J., Entin, E.E., \& Serfaty, D. (2004). Communication overhead: The hidden cost of team cognition. In E. Salas, S.M. Fiore (Eds), Team Cognition: Understanding the Factors that Drive Process and Performance (pp. 61-82). Washington, DC, US: American Psyhcological Association. doi: 10.1037/10690-004

MacFadden, A., Elias, L., \& Saucier, D. (2003). Males and females scan maps similarly, but give directions differently. Brain and Cognition, 53(2), 297-300.

Marks, M.A., Zaccaro, S.J., Mathieu, J.E. (2000). Performance implications of leader briefings and team-interaction training for team adaptation to novel environments. Journal of Applied Psychology, 85(6), 971-986.

Merz, S. (2014). Should we still teach analog clocks? Stories from School, AZ. Retrieved from http://www.storiesfromschoolaz.org/still-teach-analog-clocks/

McCarthy, J. (1995). Making robots conscious of their mental states. In S. Muggleton (Ed.), Machine Intelligence 15: Oxford University Press. 
Murphy, R. R., \& Burke, J. L. (2005). Up from the rubble: Lessons learned about HRI from search and rescue. In Proceedings of the Human Factors and Ergonomics Society Annual Meeting, 49 (3), 437-441 doi: 10.1177/154193120504900347

Nature (2010, June). Putting gender on the agenda. Nature, 465. doi doi:10.1038/465665a

Norman, D. A. (1983). Some observations on mental models. In D. Gentner \& A. L. Stevens (Eds.), Mental Models (pp. 7-14). Hillsdale, NJ: Lawrence Erlbaum Associates, Inc.

Novianto, R. \& Williams, M.A. (2009). The role of attention in robot self-awareness. In Robot and Human Interactive Communication, 2009. RO-MAN 2009. The $18^{\text {th }}$ IEEE International Symposium on (pp.1047-1053). IEEE.

Nguyen-Huu, P. N., Titus, J., Tilbury, D., \& Ulsoy, G. (2009). Reliability and failure in unmanned ground vehicle (UGV) (Vol. 1). GRRC Technical Report 2009.

Parush, A., Kramer, C., Foster-Hunt, T., Momtahan, K., Hunter, A., \& Sohmer, B. (2011). Communication and team situation awareness in the OR: Implications for augmentative information display. Journal of Biomedical Informatics, 44 (2011), 477-485. 
Phillips, E., Ososky, S., Swigert, B., \& Jentsch, F. (2012). Human-animal teams as an analog for future human-robot teams. Proceedings of the Human Factors and Ergonomics Society Annual Meeting, 56(1), 1553-1557.

doi:10.1177/1071181312561309

Piaget, J., \& Inhelder, B. (1956). The child's concept of space. New York: Humanities Press.

Rouse, W.B., \& Morris, N.M. (1986). On looking into the black box: Prospects and limits in the search for mental models. Psychological Bulletin, 100(3), 49-363. doi: 10.1037/0033-2909.100.3.349

Salmon, P.M., Stanton, N.A., Walker, G.H., \& Jenkins, D.P. (2009). A model of distributed situation awareness in complex collaborative environments. In P.M. Salmon, N.A. Stanton, G.H. Walker \& D.P. Jenkins (Eds.), Distributed Situation Awareness (pp.183-205). Burlington, VA: Ashgate Publishing Company.

Sanders, M.S., \& McCormick, E.J. (1993). Human Factors in Engineering and Design. New York, New York: McGraw-Hill, Inc.

Scholtz, J., Antonishek, B., \& Young, J. (2004, January). Evaluation of a human-robot interface: Development of a situational awareness methodology. In 2013 46th Hawaii International Conference on System Sciences (Vol. 5, pp. 50130c-50130c). IEEE Computer Society. 
Schuster, D. (2013). The effects of diagnostic aiding on situation awareness under robot unreliability (Unpublished doctoral dissertation). University of Central Florida, Orlando, Florida.

Schuster, D., \& Jentsch, F. (2012). Modeling and enhancing situation awareness in human-robot teams (Contract No. W911NF-10-2-0016). Orlando, FL: University of Central Florida

Schuster, D., Keebler, J. R., Zuniga, J., \& Jentsch, F. (2012). Individual differences in SA measurement and performance in human-robot teaming. In Cognitive Methods in Situation Awareness and Decision Support (CogSIMA), 2012 IEEE International Multi-Disciplinary Conference on (pp. 187-190). IEEE.

Schwarzkopf, S., Schilbach, L., Vogeley, K., \& Timmermans, B. (2014). "Making it explicit" makes a difference: Evidence for a dissociation of spontaneous and intentional level 1 perspective taking in high-functioning autism. Cognition, 131 (3), 345-354.

Shavers-Hornaday, V.L., Lynch, C.F., Burmeister, L.F., \& Torner, J.C.(2010). Whay are African Americans under-represented in medical research studies? Impediments to participation. Ethnicity \& Health, 2(1/2), 34-45.

Shelton, A. L., \& McNamara, T.P. (2004). Spatial memory and perspective taking. Memory and Cognition, 32(2), 416-426. 
Streater, J., Elias, J., Bockelman Morrow, P. B., \& Fiore, S. (2011). Towards an interdisciplinary understanding of perspective for human-robot teamwork. In Proceedings of the Human Factors and Ergonomics Society Annual Meeting, 55 (1), 1481-1485. doi: 10.1177/1071181311551308

Stevens, J. (1996). Applied multivariate statistics for the social sciences ( $\left.3^{\text {rd }} \mathrm{edn}\right)$. Mahwah, NJ: Lawrence Mifflin.

Tabachnick, B.G., \&Fidell, L.S. (2007). Using Multivariate Statistics ( $5^{\text {th }}$ edn). Boston: Pearson Education.

Talone, A.B., Phillips, E., \& Jentsch, F. (2016). Towards effective robot-to-human information sharing in distributed human-robot teams. Manuscript submitted for publication.

Thurstone, L.L. (1950). Some primary abilities in visual thinking (Rep. no. 59). Psychometric Laboratory, University of Chicago, Chicago, IL.

Trafton, G.J., Cassimatis, N.L., Bugajska, M.D., Borck, D.P., Mintz, F.E., \& Schultz, A.C. (2005). Enabling effective human-robot interaction using perspective-taking in robots. Systems, Man and Cybernetics, Part A: Systems and Humans, IEEE Transactions on, 35(4), 460-470. 
Urban, J. M., Bowers, C. A., Monday, S. D., \& Morgan, B. B. (1993). Effects of workload on communication process in decision making teams: An empirical study with implication for training. Proceedings of the Human Factors and Ergonmics Society Annual Meeting, 37(18), 1233-1237.

Webb, S. (2014). Calling time on the traditional clock? One in seven Britons can only tell time using a digital watch. The Daily Mail. Retrieved from http://www.dailymail.co.uk/sciencetech/article-2548115/Calling-time-traditionalclock-One-seven-Britons-tell-time-using-digital-watch.html

Wickens, C. D., \& Hollands, J. G. (1984). Engineering psychology and human performance. Columbus, Ohio: Charles E. Merrill Publishing Co.

Yanco, H. A, \& Drury, J.L. (2004)." Where Am I?" Acquiring situation awareness using a remote robot platform. Paper presented at the Systems, Man and Cybernetics, 2004 IEEE International Conference on (pp. 2835-2840). IEEE.

Yanco, H. A., \& Drury, J. L. (2007). Rescuing interfaces: A multi-year study of humanrobot interaction at the AAAI Robot Rescue Competition. Autonomous Robots, 22(4), $333-352$ 